\title{
Requirements for Late-Stage Hydroboration of Pyridine N-Heterocyclic Carbene Iron(0) Complexes: The Role of Ancillary Ligands
}

\author{
John J. Kiernicki, ${ }^{a}$ Matthias Zeller, ${ }^{b}$ and Nathaniel K. Szymczak ${ }^{a *}$
}

\begin{abstract}
${ }^{a}$ Department of Chemistry, University of Michigan, Ann Arbor, Michigan 48109, United States.
*Corresponding Author email: nszym@umich.edu

${ }^{\mathrm{b} H . C .}$ Brown Laboratory, Department of Chemistry, Purdue University, West Lafayette, Indiana 47907, United States
\end{abstract}


Experimental Procedures

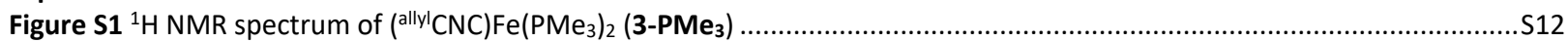

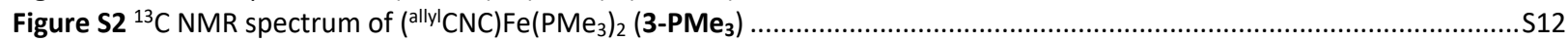

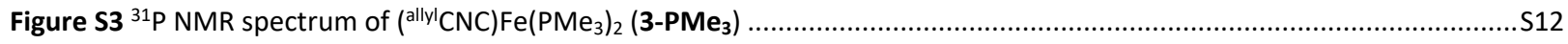

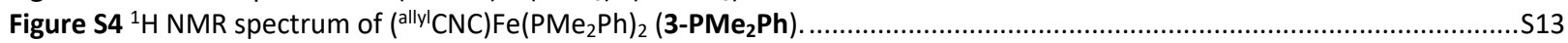

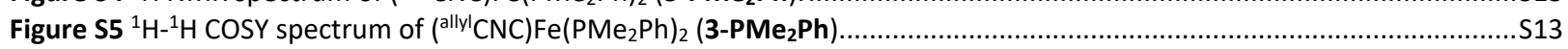

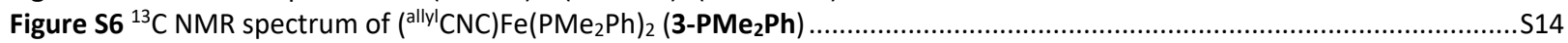

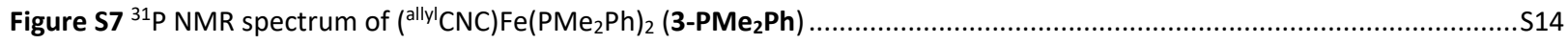

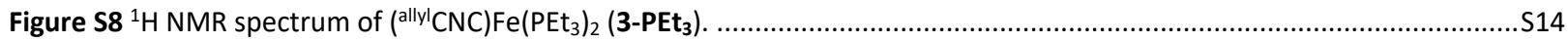

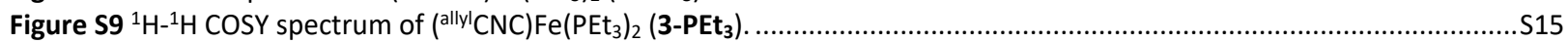

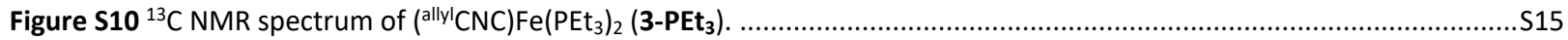

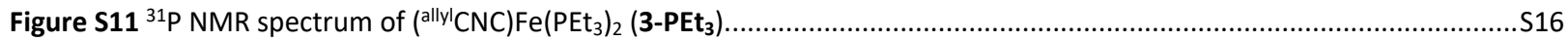

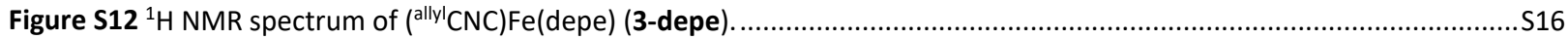

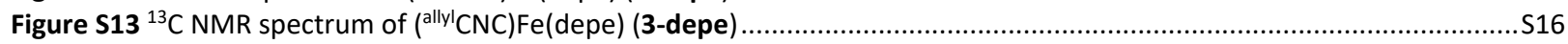

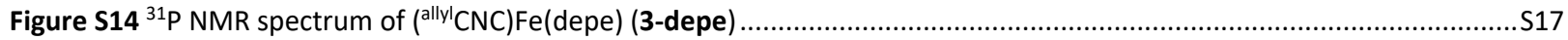

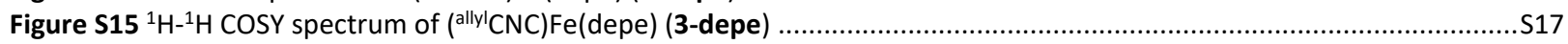

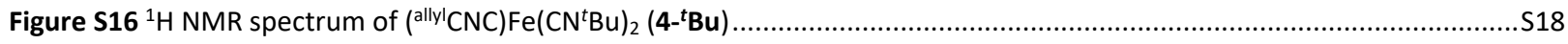

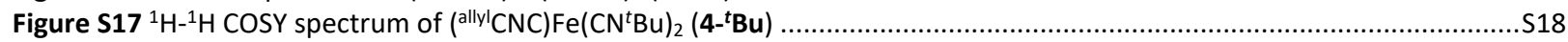

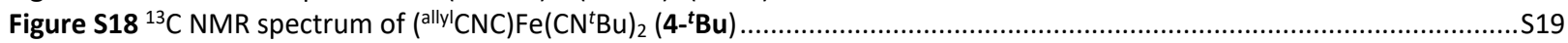

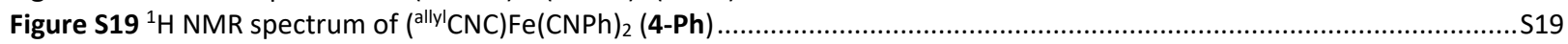

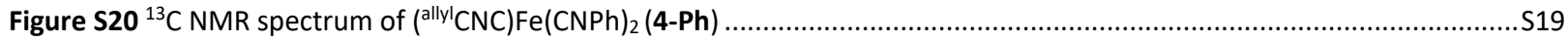

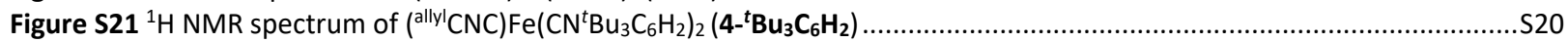

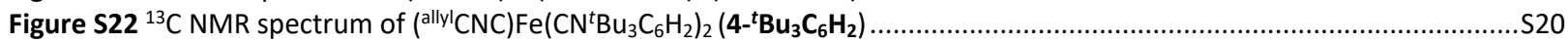

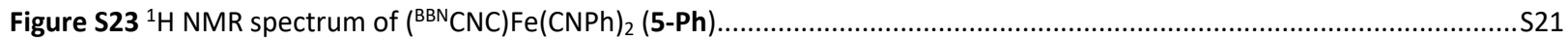

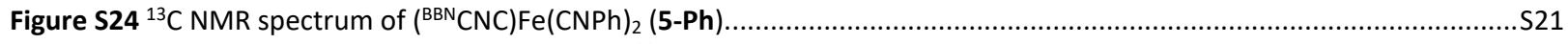

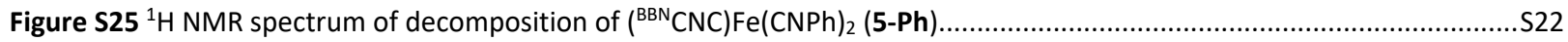

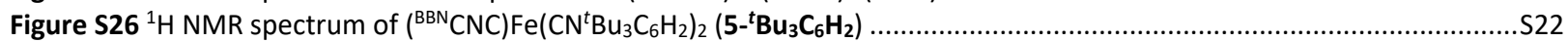

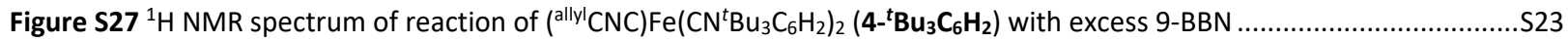

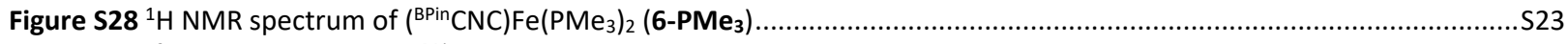

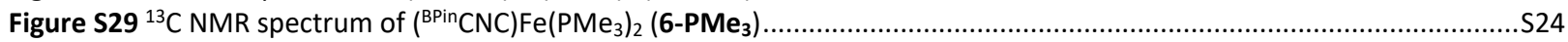

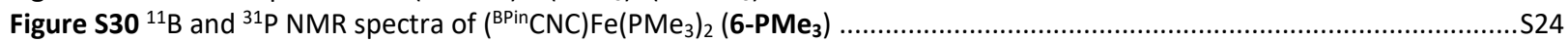

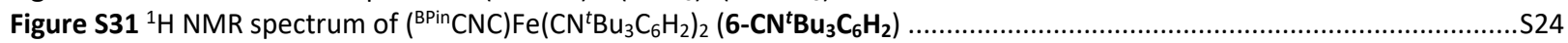

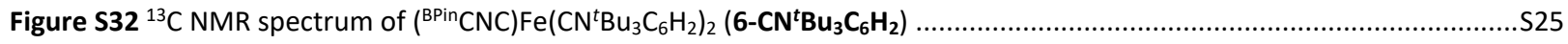

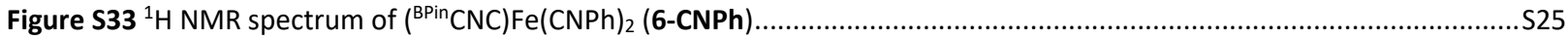

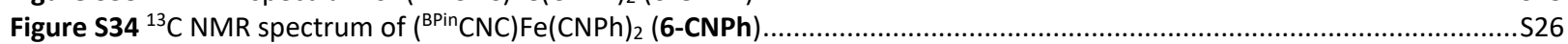

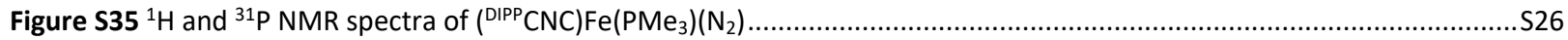

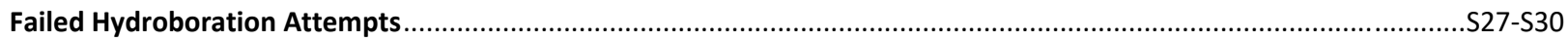

Figure $\mathbf{S} 36^{1} \mathrm{H}$ and ${ }^{31} \mathrm{P}$ NMR spectra of attempted hydroboration of (allyl $\left.\mathrm{CNC}\right) \mathrm{Fe}\left(\mathrm{PMe}_{3}\right)_{2}\left(3-\mathrm{PMe}_{3}\right)$ with 9-BBN......................S28

Figure $\mathbf{S} 37{ }^{1} \mathrm{H}$ and ${ }^{31} \mathrm{P}$ NMR spectra of attempted hydroboration of (allyl $\left.\mathrm{CNC}\right) \mathrm{Fe}\left(\mathrm{PMe} \mathrm{e}_{2} \mathrm{Ph}\right)_{2}\left(3-\mathrm{PMe} \mathrm{P}_{2} \mathrm{Ph}\right)$ with 9-BBN ................S28

Figure $\mathbf{S 3 8}{ }^{1} \mathrm{H}$ and ${ }^{31} \mathrm{P}$ NMR spectra of attempted hydroboration of (allyl CNC)Fe$\left(\mathrm{PEt}_{3}\right)_{2}\left(3-\mathrm{PEt}_{3}\right)$ with 9-BBN ..........................S29

Figure S39 ${ }^{1} \mathrm{H}$ and ${ }^{31} \mathrm{P}$ NMR spectra of attempted hydroboration of (allyl $\mathrm{CNC}$ )Fe(depe) (3-depe) with 9-BBN .........................S29

Figure $\mathbf{S 4 0}{ }^{1} \mathrm{H}$ NMR spectrum of attempted hydroboration of $\left({ }^{\text {allyl }} \mathrm{CNC}\right) \mathrm{Fe}\left(\mathrm{CN}{ }^{t} \mathrm{Bu}\right)_{2}\left({ }^{\left(4-{ }^{t} \mathrm{Bu}\right)}\right.$ with 9-BBN..............................S30

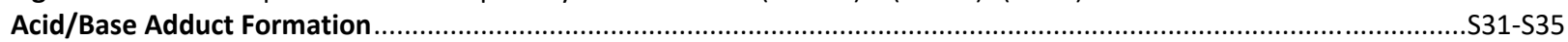

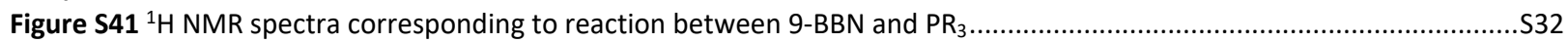

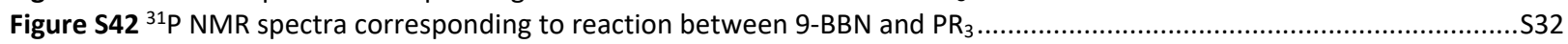

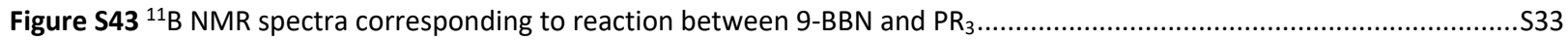

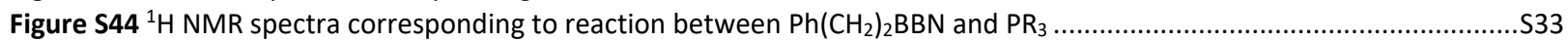

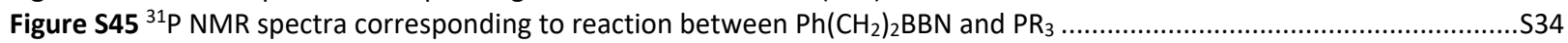

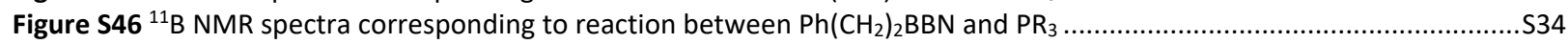

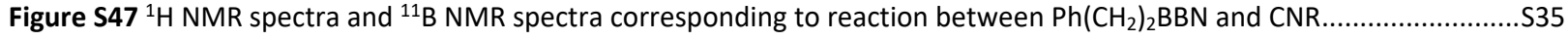

Figure S48 ${ }^{1} \mathrm{H}$ NMR spectra and ${ }^{11} \mathrm{~B}$ NMR spectra corresponding to reaction between 9-BBN and CNR ................................S35

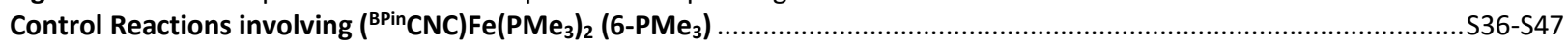

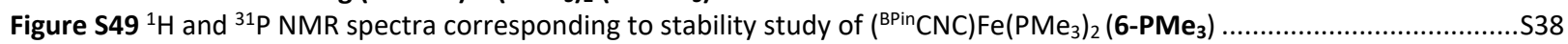

Figure S50 NMR spectra for reaction between $\mathrm{Ph}\left(\mathrm{CH}_{2}\right)_{2} \mathrm{BBN}$ and $\left({ }^{\mathrm{BPin}} \mathrm{CNC}\right) \mathrm{Fe}\left(\mathrm{PMe}_{3}\right)_{2}\left(6-\mathrm{PMe}_{3}\right)$ in $\mathrm{C}_{6} \mathrm{D}_{6} \ldots \ldots \ldots \ldots \ldots \ldots \ldots \ldots \ldots . . . . . . . . . . . . . . . .339$

Figure S51 NMR spectra for reaction between $\mathrm{Ph}\left(\mathrm{CH}_{2}\right)_{2} \mathrm{BBN}$ and $\left({ }^{\mathrm{BPin} C N C}\right) \mathrm{Fe}\left(\mathrm{PMe}_{3}\right)_{2}\left(6-\mathrm{PMe}_{3}\right)$ in $\mathrm{C}_{6} \mathrm{D}_{6}-$ static vacuum ...........S40

Figure S52 NMR spectra for reaction between $\mathrm{Ph}\left(\mathrm{CH}_{2}\right)_{2} \mathrm{BBN}$ (2 equiv.) and ( $\left.{ }^{\mathrm{BPin}} \mathrm{CNC}\right) \mathrm{Fe}\left(\mathrm{PMe}_{3}\right)_{2}\left(6-\mathrm{PMe}_{3}\right)$ in $\mathrm{C}_{6} \mathrm{D}_{6} \ldots \ldots \ldots \ldots \ldots . . . . . . . . . . .541$

Figure S53 NMR spectra for reaction between $\mathrm{Ph}\left(\mathrm{CH}_{2}\right)_{2} \mathrm{BBN}$ (2 equiv.) and ( $\left.{ }^{\mathrm{BPin}} \mathrm{CNC}\right) \mathrm{Fe}\left(\mathrm{PMe}_{3}\right)_{2}\left(6-\mathrm{PMe}_{3}\right)$ in $\mathrm{C}_{6} \mathrm{H}_{12} \ldots \ldots \ldots \ldots \ldots . . . . . . . . . .542$

Figure S54 NMR spectra for reaction between 9-BBN (2 equiv.) and $\left({ }^{\mathrm{BPin}} \mathrm{CNC}\right) \mathrm{Fe}\left(\mathrm{PMe}_{3}\right)_{2}\left(6-\mathrm{PMe}_{3}\right)$ in $\mathrm{C}_{6} \mathrm{H}_{12} \ldots \ldots \ldots \ldots \ldots \ldots \ldots \ldots . . . . . . . . . . . . . .343$ 
Figure S55 NMR spectra for protonation of $\left({ }^{\mathrm{BPin}} \mathrm{CNC}\right) \mathrm{Fe}\left(\mathrm{PMe}_{3}\right)_{2}\left(6-\mathrm{PMe}_{3}\right)$ to generate $\left[\left({ }^{\mathrm{BPin}} \mathrm{CNC}\right) \mathrm{Fe}(\mathrm{PMe})_{2}{ }_{2} \mathrm{H}\right]^{+}$- initial.............S44 Figure S56 NMR spectra for protonation of $\left({ }^{\mathrm{BPin} C N C}\right) \mathrm{Fe}\left(\mathrm{PMe}_{3}\right)_{2}\left(6-\mathrm{PMe}_{3}\right)$ to generate $\left.\left[\left({ }^{\mathrm{BPin}} \mathrm{CNC}\right) \mathrm{Fe}(\mathrm{PMe})_{3}\right)_{2} \mathrm{H}\right]^{+}-$final...............S45

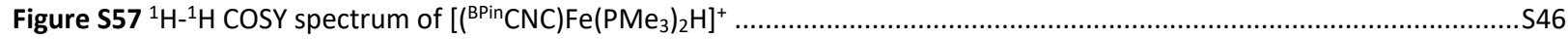

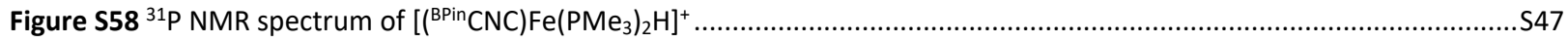

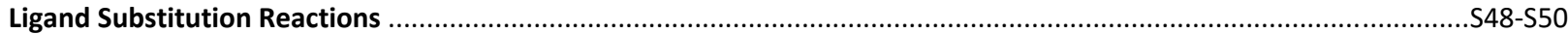

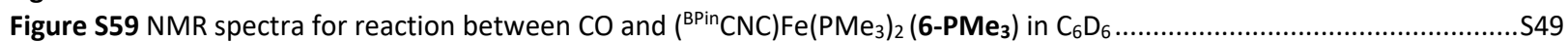

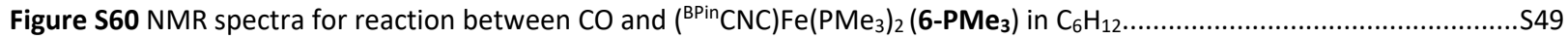

Figure $\mathbf{S 6 1}{ }^{1} \mathrm{H}$ NMR spectra for reaction between ${ }^{\mathrm{B}} \mathrm{BuNC}$ and $\left({ }^{\mathrm{BPin}} \mathrm{CNC}\right) \mathrm{Fe}\left(\mathrm{PMe}_{3}\right)_{2}\left(\mathbf{6}-\mathrm{PMe}_{3}\right)$ followed by CO...........................S50

Figure $\mathbf{S 6 2}{ }^{31} \mathrm{P}$ NMR spectra for reaction between ${ }^{\mathrm{t}} \mathrm{BuNC}$ and $\left({ }^{\mathrm{BPin}} \mathrm{CNC}\right) \mathrm{Fe}\left(\mathrm{PMe}_{3}\right)_{2}\left(6-\mathrm{PMe}_{3}\right)$ followed by $\mathrm{CO} \ldots \ldots \ldots \ldots \ldots \ldots \ldots \ldots . . . . . . . . . . . . . .550$

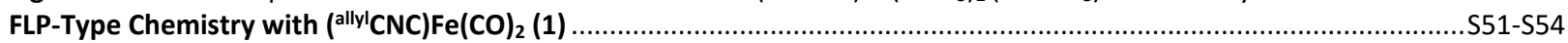

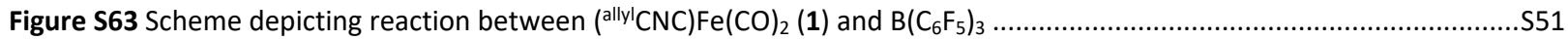

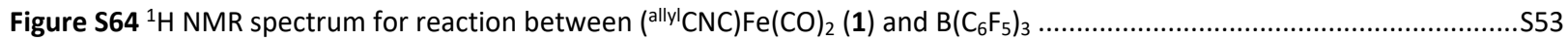

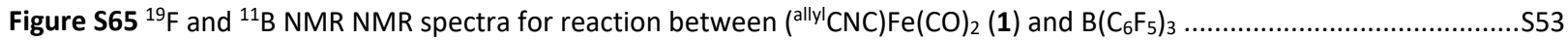

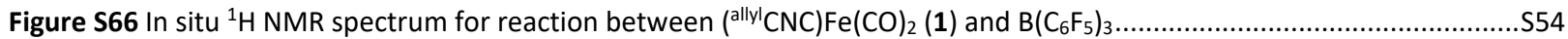

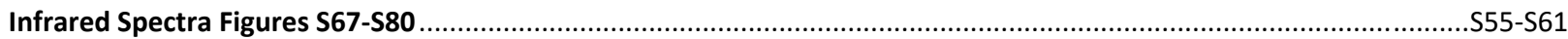

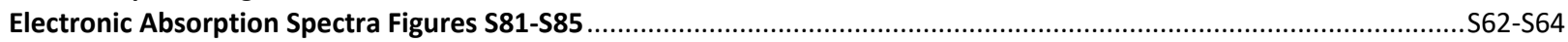

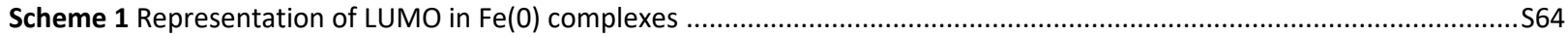

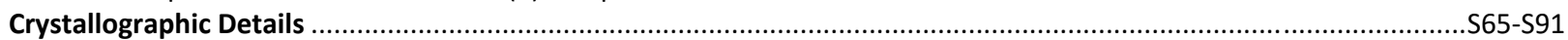

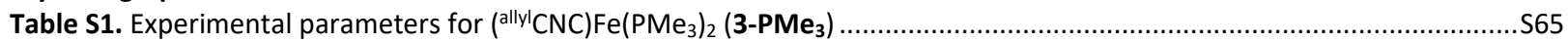

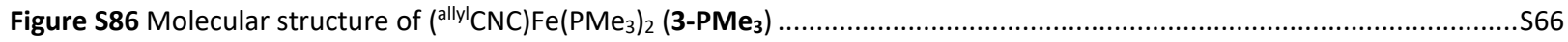

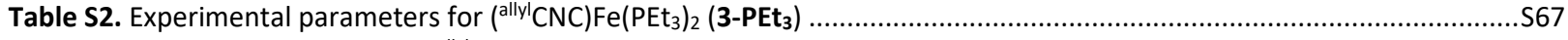

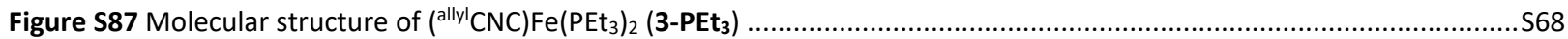

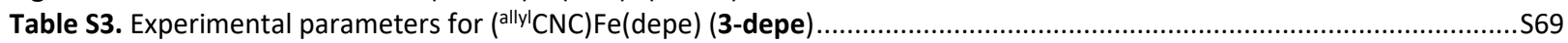

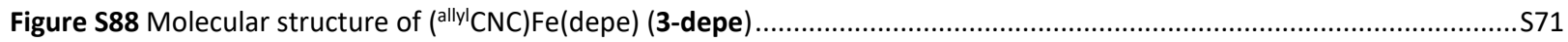

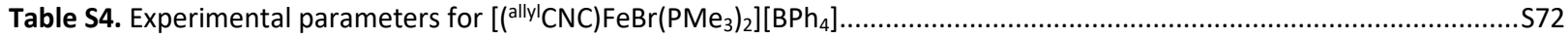

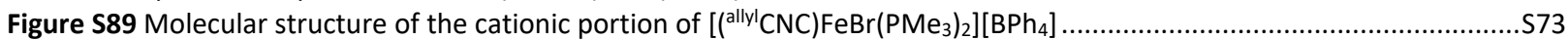

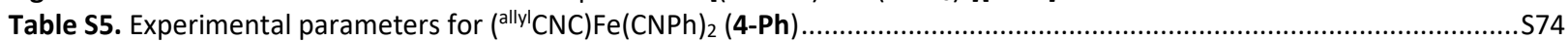

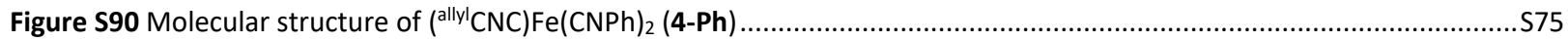

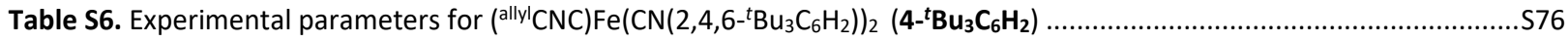

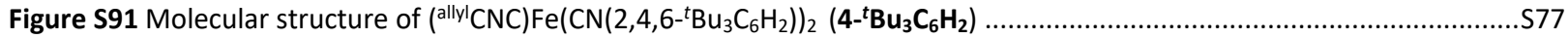

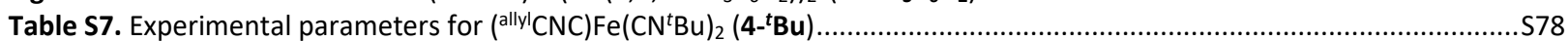

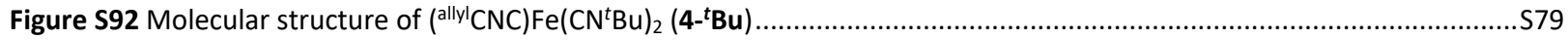

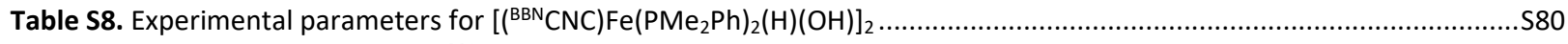

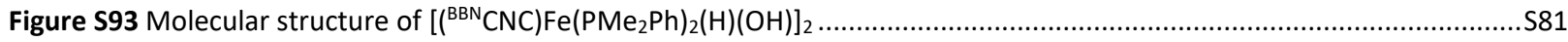

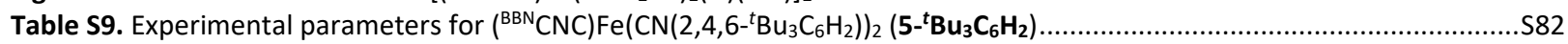

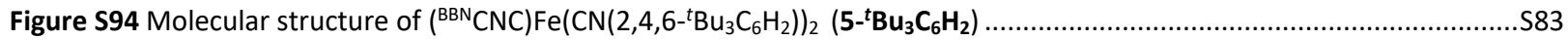

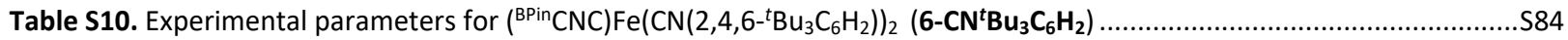

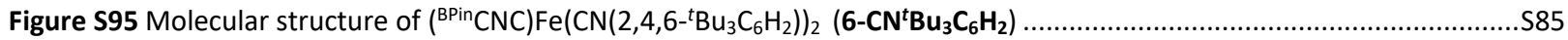

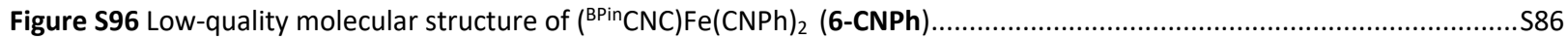

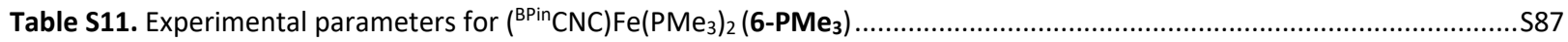

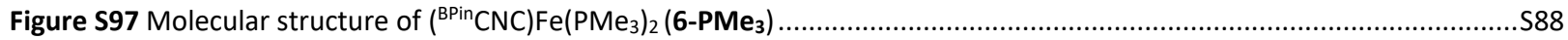

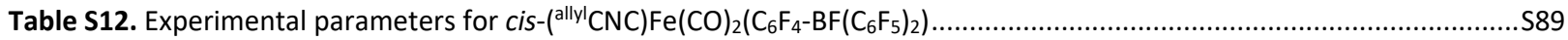

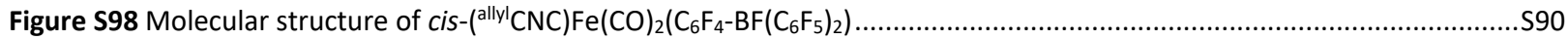

Table S13. Experimentally determined bond distances and angles for phosphine containing complexes ................................S91

Table S14. Experimentally determined bond distances and angles for isocyanide containing complexes ..............................S91

References 
General Considerations. All air- and moisture-sensitive manipulations were performed using standard Schlenk techniques or in an inert atmosphere drybox with an atmosphere of purified nitrogen. The drybox was equipped with a cold well designed for freezing samples in liquid nitrogen as well as a $-35^{\circ} \mathrm{C}$ freezer for cooling samples and crystallizations. Solvents were purified using a Glass Contour solvent purification system through percolation through a $\mathrm{Cu}$ catalyst, molecular sieves, and alumina. Solvents were then stored over sodium and/or sieves. Benzene- $d_{6}$ was purchased from Cambridge Isotope Laboratories, dried with molecular sieves and sodium, and degassed by three freeze-pump-thaw cycles.

All phosphines, tert-butylisocyanide, 9-borabicyclo[3.3.1]nonane, and $B\left(C_{6} F_{5}\right)_{3}$ were purchased from commercial vendors and used as received. $\left[\left({ }^{\text {allyly }} \mathrm{CNC}\right) \mathrm{Fe}(\mathrm{MeCN})_{3}\right]\left[\mathrm{BPh}_{4}\right]_{2}(2){ }_{1}^{1}\left[\left({ }^{\mathrm{BPin}} \mathrm{CNC}\right) \mathrm{Fe}(\mathrm{MeCN})_{3}\right]\left[\mathrm{BPh}_{4}\right]_{2},{ }^{1}$ $\left({ }^{\text {allyl }} \mathrm{CNC}\right) \mathrm{Fe}(\mathrm{CO})_{2}$ (1), ${ }^{1}$ potassium graphite, ${ }^{2}$ 2,4,6-tri-tert-butylphenylisocyanide, ${ }^{3}$ phenylisocyanide, ${ }^{3}$ $\mathrm{HB}\left(\mathrm{C}_{6} \mathrm{~F}_{5}\right)_{2},{ }^{4}\left[\mathrm{H}\left(\mathrm{OEt}_{2}\right)_{2}\right]\left[\mathrm{BAr}_{24}{ }_{24},{ }^{5}\right.$ and $\mathrm{Ph}\left(\mathrm{CH}_{2}\right)_{2} \mathrm{BBN}^{6}$ were synthesized according to literature procedures.

NMR spectra were recorded on Varian spectrometers. ${ }^{1} \mathrm{H},{ }^{13} \mathrm{C},{ }^{31} \mathrm{P}$, and ${ }^{11} \mathrm{~B}$ chemical shifts are reported in parts per million (ppm) relative to tetramethylsilane and referenced internally to the residual solvent peak. ${ }^{11} \mathrm{~B}$ spectra were referenced on a unified scale, where the single primary reference is the frequency of the residual solvent peak in the ${ }^{1} \mathrm{H}$ NMR spectrum. ${ }^{31} \mathrm{P}$ spectra $(202 \mathrm{MHz})$ are referenced vs. $\mathrm{H}_{3} \mathrm{PO}_{4} \cdot{ }^{11} \mathrm{~B}$ spectra are referenced vs. $\mathrm{BF}_{3}\left(\mathrm{OEt}_{2}\right)$. Multiplicities are reported as follows: singlet (s), doublet (d), triplet $(\mathrm{t})$, quartet (q). Infrared spectra were recorded using a Nicolet iS10 FT-IR spectrometer. Samples were diluted into dry $\mathrm{KBr}$ and recorded as pellets. MALDI-TOF experiments were collected on a Bruker AutoFlex Speed instrument. Data were collected from an anthracene matrix using the lowest power setting possible. Samples were prepared by diluting the sample into a concentrated anthracene solution in THF (ca. $0.0001 \mathrm{~g}$ sample in $0.5 \mathrm{~mL}$ solution), spotting the solution (ca. $0.001 \mathrm{~mL}$ ) on a Bruker sample plate, and allowing the solution to evaporate to dryness. High resolution liquid chromatography mass spectrometry data (HR LC-MS) were obtained on an Agilent 6230 TOF instrument. Samples were prepared in an inert atmosphere by diluting ca. $0.0001 \mathrm{~g}$ sample in $15 \mathrm{~mL}$ THF. Samples were collected in positive ion mode.

Single crystals of $\quad\left({ }^{\text {allyl }} \mathrm{CNC}\right) \mathrm{Fe}\left(\mathrm{PMe}_{3}\right)_{2} \quad$ (3-PMe $)_{3}, \quad\left({ }^{\mathrm{BPin}} \mathrm{CNC}\right) \mathrm{Fe}\left(\mathrm{PMe}_{3}\right)_{2} \quad$ (6-PMe $\left.\mathbf{P}_{3}\right), \quad$ and $\left[\left({ }^{\text {allyl }} \mathrm{CNC}\right) \mathrm{FeBr}\left(\mathrm{PMe}_{3}\right)_{2}\right]\left[\mathrm{BPh}_{4}\right]$ suitable for X-ray diffraction were coated with poly(isobutylene) oil in a drybox and mounted on a Rigaku AFC10K Saturn 944+ CCD-based X-ray diffractometer equipped with a low temperature device and a Micromax-007HF Cu-target microfocus rotating anode $(\lambda=1.54187 \AA)$. The data were collected using CrystalClear 2.011 and processed using CrysAlis PRO 1.171.38.41. Empirical absorption correction was applied using spherical harmonics, as implemented in the SCALE3 ABSPACK scaling algorithm. Single crystals of $\left({ }^{\text {allyly }} \mathrm{CNC}\right) \mathrm{Fe}\left(\mathrm{PEt}_{3}\right)_{2} \quad$ (3-PEt $\left.{ }_{3}\right), \quad\left({ }^{\text {ally }} \mathrm{CNC}\right) \mathrm{Fe}$ (depe) (3-depe), $\left({ }^{B P i n} \mathrm{CNC}\right) \mathrm{Fe}\left(\mathrm{CN}^{t} \mathrm{Bu}_{3} \mathrm{C}_{6} \mathrm{H}_{2}\right)_{2}, \quad\left(6-\mathrm{CN}^{t} \mathrm{Bu}_{3} \mathrm{C}_{6} \mathrm{H}_{2}\right)$ and cis-( $\left.{ }^{\text {allylyl}} \mathrm{CNC}\right) \mathrm{Fe}(\mathrm{CO})_{2}\left(\mathrm{C}_{6} \mathrm{~F}_{4}-\mathrm{BF}\left(\mathrm{C}_{6} \mathrm{~F}_{5}\right)_{2}\right)$ suitable for $\mathrm{X}$-ray diffraction were coated with poly(isobutylene) oil and quickly transferred to the goniometer head of a Bruker AXS D8 Quest diffractometer with a fixed chi angle, a sealed tube fine focus X-ray tube, single crystal curved graphite incident beam monochromator and a Photon100 or a Photon2 CMOS area detector. Examination and data collection were performed with Mo Ka radiation $(\lambda=0.71073 \AA)$. Single crystals of (allyl $\mathrm{CNC}) \mathrm{Fe}\left(\mathrm{CN}^{t} \mathrm{Bu}_{3} \mathrm{C}_{6} \mathrm{H}_{2}\right)_{2}\left({ }^{4}-{ }^{t} \mathrm{Bu}_{3} \mathrm{C}_{6} \mathrm{H}_{2}\right),\left({ }^{\mathrm{BBN}} \mathrm{CNC}\right) \mathrm{Fe}\left(\mathrm{CN}^{t} \mathrm{Bu}_{3} \mathrm{C}_{6} \mathrm{H}_{2}\right)_{2}\left({ }^{-}-{ }^{t} \mathrm{Bu}_{3} \mathrm{C}_{6} \mathrm{H}_{2}\right)$, (allyl $\left.\mathrm{CNC}\right) \mathrm{Fe}(\mathrm{CNPh})_{2}$ (4-Ph), (allyl $\mathrm{CNC}) \mathrm{Fe}\left(\mathrm{CN}^{t} \mathrm{Bu}\right)_{2}\left({ }^{4}-{ }^{t} \mathrm{Bu}\right)$, and $\left[\left({ }^{\mathrm{BBN}} \mathrm{CNC}\right) \mathrm{Fe}\left(\mathrm{PMe}{ }_{2} \mathrm{Ph}\right)_{2}(\mathrm{H})(\mathrm{OH})\right]_{2}$ suitable for $\mathrm{X}$-ray diffraction were coated with poly(isobutylene) oil and quickly transferred to the goniometer head of a Bruker AXS D8 Quest diffractometer with kappa geometry, an I- $\mu$-S microsource X-ray tube, laterally graded multilayer (Goebel) mirror for monochromatization, a Photon2 or a Photon3 CMOS area detector and an Oxford Cryosystems low temperature device. Examination and data collection were performed with $\mathrm{Cu} K \alpha$ radiation $(\lambda=$ 
$1.54184 \AA$ A). For both Quest instruments, data were collected, reflections were indexed and processed, and the files scaled and corrected for absorption using APEX3, SAINT and SADABS. ${ }^{7}$ For all samples, the space groups were assigned using XPREP within the SHELXTL suite of programs ${ }^{8}$ and the structures were solved by direct methods using ShelXS- $97^{9}$ and refined by full matrix least squares against $\mathrm{F}^{2}$ with all reflections using Shelx|2014 (Rigaku samples), Shelx|2017 or Shelx|2018 ${ }^{10}$ using the graphical interface Shelxle. ${ }^{11}$ If not specified otherwise, $\mathrm{H}$ atoms attached to carbon atoms were positioned geometrically and constrained to ride on their parent atoms, with carbon hydrogen bond distances of $0.95 \AA$ for and aromatic $\mathrm{C}-\mathrm{H}, 1.00,0.99$ and $0.98 \AA$ for aliphatic $\mathrm{C}-\mathrm{H}, \mathrm{CH}_{2}$, and $\mathrm{CH}_{3}$ moieties, respectively. Methyl $\mathrm{H}$ atoms were allowed to rotate but not to tip to best fit the experimental electron density. Hydroxyl and hydride $\mathrm{H}$ atom positions and thermal parameters were freely refined. $\mathrm{U}_{\text {iso }}(\mathrm{H})$ values were set to a multiple of $\mathrm{U}_{\text {eq }}(\mathrm{C})$ with 1.5 for $\mathrm{CH}_{3}$, and 1.2 for $\mathrm{CH}_{2}$, and $\mathrm{C}-\mathrm{H}$ units, respectively. Additional data collection and refinement details, including description of disorder (where present) can be found with the individual structure descriptions, below. Complete crystallographic data, in CIF format, have been deposited with the Cambridge Crystallographic Data Centre. CCDC 2084280-2084291 contains the supplementary crystallographic data for this paper. These data can be obtained free of charge from The Cambridge Crystallographic Data Centre via www.ccdc.cam.ac.uk/data_request/cif.

\section{Synthesis of Compounds}

Synthesis of (allyl $\mathrm{CNC}) \mathrm{Fe}\left(\mathrm{PMe}_{3}\right)_{2} \quad$ (3-PMe $)_{3}$. A $20 \mathrm{~mL}$ scintillation vial was charged with $\left[\left({ }^{\text {allyl }} \mathrm{CNC}\right) \mathrm{Fe}(\mathrm{MeCN})_{3}\right]\left[\mathrm{BPh}_{4}\right]_{2}$ (2) $(0.200 \mathrm{~g}, 0.181 \mathrm{mmol})$ and $10 \mathrm{~mL} \mathrm{THF}$. While stirring, trimethylphosphine $(0.039 \mathrm{~mL}, 0.377 \mathrm{mmol})$ was added resulting in a color change from light orange to orange-red. The slurry was then frozen. Upon thawing, potassium graphite $(0.050 \mathrm{~g}, 0.370 \mathrm{mmol})$ was added resulting in a rapid color change to deep brown. The reaction was stirred for $20 \mathrm{~min}$ and volatiles were removed in vacuo. The mixture was extracted with $15 \mathrm{~mL}$ diethyl ether, filtered, and dried to afford dark brown-black powder $(0.080 \mathrm{~g}, 0.160 \mathrm{mmol}, 86 \%)$ assigned as $\left({ }^{\mathrm{BPin}} \mathrm{CNC}\right) \mathrm{Fe}\left(\mathrm{PMe}_{3}\right)_{2}\left(\mathbf{3}-\mathrm{PMe}_{3}\right)$. Single, $\mathrm{X}$-ray quality crystals were obtained from a concentrated diethyl ether solution stored at $-35^{\circ} \mathrm{C}$. HR LC-MS of [M]+H $\left(\mathrm{C}_{23} \mathrm{H}_{35} \mathrm{~N}_{5} \mathrm{P}_{2} \mathrm{Fe}_{1}\right.$ $+\mathrm{H}$ ): calc. 500.1795; found 500.1779. ${ }^{1} \mathrm{H} N M R\left(\mathrm{C}_{6} \mathrm{D}_{6}, 25{ }^{\circ} \mathrm{C}, 500 \mathrm{MHz}\right) \delta=0.91\left(\mathrm{t}, J=2.3,18 \mathrm{H}, \mathrm{P}\left(\mathrm{CH}_{3}\right)_{3}\right)$, $5.12\left(\mathrm{~d}, J=6.2,4 \mathrm{H}, \mathrm{N}-\mathrm{CH}_{2}\right), 5.14(\mathrm{~d}, J=10.5,2 \mathrm{H}, \mathrm{CH}=\mathrm{CHH}), 5.26$ (dq, $J=17.1,1.7,2 \mathrm{H}, \mathrm{CH}=\mathrm{CHH}$ ), 6.12 (ddt, $\left.J=16.5,10.1,6.1,2 \mathrm{H}, \mathrm{CH}=\mathrm{CH}_{2}\right), 6.97(\mathrm{~d}, J=2.1,2 \mathrm{H}, \mathrm{IM}-\mathrm{CH}), 7.32$ (overlapping resonances, $3 \mathrm{H}, m$ - and $p$ pyr- $\mathrm{CH}$ ), $7.73(\mathrm{~d}, J=2.1,2 \mathrm{H}, \mathrm{IM}-\mathrm{CH}) .{ }^{13} \mathrm{C} \mathrm{NMR}\left(\mathrm{C}_{6} \mathrm{D}_{6}, 25{ }^{\circ} \mathrm{C}, 126 \mathrm{MHz}\right) \delta=27.57\left(\mathrm{t}, J=8.0, \mathrm{P}\left(\mathrm{CH}_{3}\right)_{3}\right), 53.93$ $\left(\mathrm{N}-\mathrm{CH}_{2}\right), 96.91,107.26,111.09,118.57,119.76,135.34,141.82$ (p-pyr-CH), 214.39 (t, $J=19.4$, carbene- $\mathrm{C}$ ).

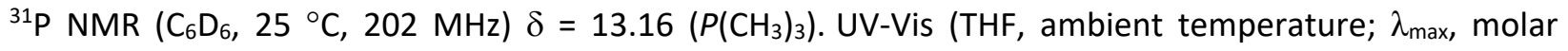
absorptivity): $333 \mathrm{~nm}, 7700 \mathrm{M}^{-1} \mathrm{~cm}^{-1} ; 416 \mathrm{~nm}, 13700 \mathrm{M}^{-1} \mathrm{~cm}^{-1}$. Selected IR data $(\mathrm{KBr}) \mathrm{v}=2957,2892,1471$, $1406,1252,1223,928,620,655 \mathrm{~cm}^{-1}$. 
Synthesis of (allyl $\mathrm{CNC}) \mathrm{Fe}\left(\mathrm{PEt}_{3}\right)_{2} \quad$ (3-PEt $\left.)_{3}\right) . \quad$ A $20 \mathrm{~mL}$ scintillation vial was charged with $\left[\left({ }^{\text {allyl }} \mathrm{CNC}\right) \mathrm{Fe}(\mathrm{MeCN})_{3}\right]\left[\mathrm{BPh}_{4}\right]_{2}$ (2) $(0.250 \mathrm{~g}, 0.226 \mathrm{mmol})$ and $10 \mathrm{~mL}$ THF. While stirring, triethylphosphine $(0.067 \mathrm{~mL}, 0.455 \mathrm{mmol}$ ) was added to the suspension resulting in a slight color change from light orange to a darker shade of orange. The mixture was then frozen. Upon thawing, potassium graphite $(0.061 \mathrm{~g}$, $0.451 \mathrm{mmol}$ ) was added to the slurry resulting in a rapid color charge to dark brown. The mixture was stirred at room temperature for $10 \mathrm{~min}$, filtered, and dried. The resulting material was extracted into 10 $\mathrm{mL}$ diethyl ether, filtered, and dried to afford brown powder $(0.088 \mathrm{~g}, 0.151 \mathrm{mmol}, 67 \%)$ assigned as (allyl $\mathrm{CNC}) \mathrm{Fe}\left(\mathrm{PEt}_{3}\right)_{2}\left(3-\mathrm{PEt}_{3}\right)$. Single, $\mathrm{X}$-ray quality crystals were obtained by slow evaporation of a $n$-pentane solution of (allyl CNC)Fe( $\left.\mathrm{PEt}_{3}\right)_{2}\left(3-\mathrm{PEt}_{3}\right)$ at $-35^{\circ} \mathrm{C}$. MALDI-TOF and HR LC-MS experiments failed to produce an assignable ion peak. ${ }^{1} \mathrm{H}$ NMR $\left(\mathrm{C}_{6} \mathrm{D}_{6}, 25{ }^{\circ} \mathrm{C}, 700 \mathrm{MHz}\right) \delta=0.30\left(\mathrm{~s}, 18 \mathrm{H}, \mathrm{PCH}_{2} \mathrm{CH}_{3}\right), 1.19\left(\mathrm{~s}, 12 \mathrm{H}, \mathrm{PCH}_{2} \mathrm{CH}_{3}\right)$, $5.13(\mathrm{~d}, J=10.2,2 \mathrm{H}, \mathrm{CH}=\mathrm{CHH}), 5.22\left(\mathrm{~s}\right.$, broad, $\left.4 \mathrm{H}, \mathrm{N}-\mathrm{CH}_{2}\right), 5.25(\mathrm{~d}, J=17.2,2 \mathrm{H}, \mathrm{CH}=\mathrm{CHH}), 6.11$ (ddt, $J=$ $16.3,11.0,5.9,2 \mathrm{H}, \mathrm{CH}=\mathrm{CH}_{2}$ ), $6.97(\mathrm{~s}, 2 \mathrm{H}, \mathrm{IM}-\mathrm{CH}), 7.40$ (overlapping resonances, $3 \mathrm{H}, m$ - and $p$-pyr- $\mathrm{CH}$ ), 7.75 $(\mathrm{s}, 2 \mathrm{H}, \mathrm{IM}-\mathrm{CH}) .{ }^{13} \mathrm{C} \mathrm{NMR}\left(\mathrm{C}_{6} \mathrm{D}_{6}, 25{ }^{\circ} \mathrm{C}, 126 \mathrm{MHz}\right) \delta=7.70\left(\mathrm{PCH}_{2} \mathrm{CH}_{3}\right), 27.57\left(\mathrm{PCH}_{2} \mathrm{CH}_{3}\right), 54.12\left(\mathrm{~N}-\mathrm{CH}_{2}\right), 97.42$, 107.03, 111.14, 118.32, 120.14, 135.43, 142.10 (p-pyr-CH), $212.73(\mathrm{t}, J=19.3$, carbene- $C) .{ }^{31} \mathrm{P} N M R\left(\mathrm{C}_{6} \mathrm{D}_{6}\right.$, $\left.25^{\circ} \mathrm{C}, 202 \mathrm{MHz}\right) \delta=45.34\left(P\left(\mathrm{CH}_{2} \mathrm{CH}_{3}\right)_{3}\right)$. UV-Vis (THF, ambient temperature; $\lambda_{\max }$, molar absorptivity): 357 $\mathrm{nm}, 11000 \mathrm{M}^{-1} \mathrm{~cm}^{-1} ; 439 \mathrm{~nm}, 15000 \mathrm{M}^{-1} \mathrm{~cm}^{-1}$. Selected IR data $(\mathrm{KBr}) \mathrm{v}=1471,1404,1251,1222,761,721$, $686,661 \mathrm{~cm}^{-1}$.

Synthesis of (allyl $\mathrm{CNC}) \mathrm{Fe}\left(\mathrm{PMe}_{2} \mathrm{Ph}\right)_{2}$ (3-PMe $\mathrm{Ph}_{2} \mathrm{Ph}$. A $20 \mathrm{~mL}$ scintillation vial was charged with $\left[\left({ }^{(a l y l} \mathrm{CNC}\right) \mathrm{Fe}(\mathrm{MeCN})_{3}\right]\left[\mathrm{BPh}_{4}\right]_{2} \quad(2) \quad(0.250 \mathrm{~g}, 0.226 \mathrm{mmol})$ and $10 \mathrm{~mL}$ THF. While stirring, dimethylphenylphosphine $(0.065 \mathrm{~mL}, 0.457 \mathrm{mmol})$ was added to the suspension resulting in a slight color change from light orange to a darker shade of orange. The mixture was then frozen. Upon thawing, potassium graphite $(0.061 \mathrm{~g}, 0.451 \mathrm{mmol})$ was added to the slurry resulting in a rapid color charge to dark brown. The mixture was stirred at room temperature for $10 \mathrm{~min}$, filtered, and dried. The resulting material was extracted into $10 \mathrm{~mL}$ diethyl ether, filtered, and dried to afford brown powder $(0.107 \mathrm{~g}, 0.172 \mathrm{mmol}$, $76 \%)$ assigned as (allyl CNC)Fe(PMe $2 \mathrm{Ph})_{2}$ (3-PMe $\left.{ }_{2} \mathrm{Ph}\right)$. HR LC-MS of [M]+H $\left(\mathrm{C}_{33} \mathrm{H}_{39} \mathrm{~N}_{5} \mathrm{P}_{2} \mathrm{Fe}_{1}+\mathrm{H}\right)$ : calc. 624.2108; found 624.2131. MALDI-TOF of [M] ${ }^{+}\left(\mathrm{C}_{33} \mathrm{H}_{39} \mathrm{~N}_{5} \mathrm{P}_{2} \mathrm{Fe}_{1}\right)$ : calc. 623.2030; found 623.688. ${ }^{1} \mathrm{H} \mathrm{NMR}$ $\left(\mathrm{C}_{6} \mathrm{D}_{6}, 25{ }^{\circ} \mathrm{C}, 700 \mathrm{MHz}\right) \delta=1.26(\mathrm{~s}, 12 \mathrm{H}, \mathrm{PCH}), 4.59\left(\mathrm{~d}, J=6.2,4 \mathrm{H}, \mathrm{N}-\mathrm{CH}_{2}\right), 4.98(\mathrm{dd}, J=10.2,1.9,2 \mathrm{H}$, $\mathrm{CH}=\mathrm{CHH}), 5.06(\mathrm{dd}, J=17.2,2.02 \mathrm{H}, \mathrm{CH}=\mathrm{CHH}), 5.38\left(\mathrm{ddt}, J=16.6,10.1,6.3,2 \mathrm{H}, \mathrm{CH}=\mathrm{CH}_{2}\right), 6.61(\mathrm{~s}, 4 \mathrm{H}, \mathrm{Ph}-$ $\mathrm{CH}), 6.68(\mathrm{~d}, J=2.0,2 \mathrm{H}, \mathrm{IM}-\mathrm{CH}$ ), 6.85-6.88 (overlapping resonances, $6 \mathrm{H}, \mathrm{Ph}-\mathrm{CH}$ ), 7.39-7.43 (overlapping resonances, $3 \mathrm{H}, m$ - and $p$-pyr-CH), $7.66(\mathrm{~d}, J=1.9,2 \mathrm{H}, \mathrm{IM}-\mathrm{CH}) .{ }^{13} \mathrm{C} \mathrm{NMR}\left(\mathrm{C}_{6} \mathrm{D}_{6}, 25{ }^{\circ} \mathrm{C}, 126 \mathrm{MHz}\right) \delta=26.14$ $\left(\mathrm{PCH}_{3}\right), 53.55\left(\mathrm{~N}-\mathrm{CH}_{2}\right), 97.53,108.07,110.89,118.06,120.13,126.47,127.36(\mathrm{t}, J=3.2, \mathrm{Ph}-\mathrm{C}), 129.21(\mathrm{t}, J$ $=5.4, \mathrm{Ph}-\mathrm{C}), 135.58,142.12$ ( $p$-pyr-CH), 150.77 ( $\mathrm{t}, J=10.8, \mathrm{Ph}-\mathrm{C}), 213.37$ ( $\mathrm{t}, J=18.5$, carbene- $C$ ). ${ }^{31} \mathrm{P}$ NMR $\left(\mathrm{C}_{6} \mathrm{D}_{6}, 25^{\circ} \mathrm{C}, 202 \mathrm{MHz}\right) \delta=29.85$ ( $\left.P \mathrm{PMe}_{2} \mathrm{Ph}\right)$. UV-Vis (THF, ambient temperature; $\lambda_{\max }$, molar absorptivity): $346 \mathrm{~nm}, 9700 \mathrm{M}^{-1} \mathrm{~cm}^{-1} ; 432 \mathrm{~nm}, 13500 \mathrm{M}^{-1} \mathrm{~cm}^{-1}$. Selected IR data $(\mathrm{KBr}) \mathrm{v}=2959,2895,1472,1406,1252$, $1224,1065,921,886,691,662,493 \mathrm{~cm}^{-1}$. 
Synthesis of (allyl $\mathrm{CNC}$ )Fe(depe) (3-depe). A $20 \mathrm{~mL}$ scintillation vial was charged with $\left[\left({ }^{\text {allyl }} \mathrm{CNC}\right) \mathrm{Fe}(\mathrm{MeCN})_{3}\right]\left[\mathrm{BPh}_{4}\right]_{2} \quad$ (2) $\quad(0.250 \mathrm{~g}, 0.226 \mathrm{mmol})$ and $10 \mathrm{~mL}$ THF. While stirring, 1,2bis(diethylphosphino)ethane $(0.053 \mathrm{~mL}, 0.226 \mathrm{mmol}$ ) was added to the suspension resulting in a slight color change from light orange to a darker shade of orange. The mixture was then frozen. Upon thawing, potassium graphite $(0.062 \mathrm{~g}, 0.459 \mathrm{mmol})$ was added to the slurry resulting in a rapid color charge to dark brown. The mixture was stirred at room temperature for $20 \mathrm{~min}$ then dried. The resulting material was extracted into $12 \mathrm{~mL}$ diethyl ether, filtered, and dried to afford brown powder $(0.077 \mathrm{~g}, 0.139 \mathrm{mmol}, 62 \%)$ assigned as (allyl $\mathrm{CNC}$ )Fe(depe) (3-depe). Single, X-ray quality crystals were obtained by slow evaporation of a $n$-pentane solution of (allyl $\mathrm{CNC}$ ) Fe(depe) (3-depe) stored at $-35^{\circ} \mathrm{C}$. HR LC-MS of [M] ${ }^{+}\left(\mathrm{C}_{27} \mathrm{H}_{41} \mathrm{~N}_{5} \mathrm{P}_{2} \mathrm{Fe}_{1}\right)$ : calc. 553.2187; found 553.2178. ${ }^{1} \mathrm{H}$ NMR $\left(\mathrm{C}_{6} \mathrm{D}_{6}, 25^{\circ} \mathrm{C}, 700 \mathrm{MHz}\right) \delta=0.46\left(\mathrm{dt}, J=6.5,7.3,12 \mathrm{H}, \mathrm{PCH}_{2} \mathrm{CH}_{3}\right)$, $1.13(\mathrm{dq}, J=7.6,6.9,4 \mathrm{H}, \mathrm{P}-\mathrm{CH}), 1.67(\mathrm{~m}, 4 \mathrm{H}, \mathrm{P}-\mathrm{CH}), 1.85\left(\mathrm{~m}, 4 \mathrm{H}, \mathrm{P}-\mathrm{CH}_{2} \mathrm{CH}_{2}-\mathrm{P}\right), 4.50\left(\mathrm{~d}, J=5.9,4 \mathrm{H}, \mathrm{N}-\mathrm{CH}_{2}\right)$, $5.10(\mathrm{~d}, J=10.1,2 \mathrm{H}, \mathrm{CH}=\mathrm{CHH}), 5.17(\mathrm{dd}, J=17.4,2.1,2 \mathrm{H}, \mathrm{CH}=\mathrm{CHH}), 5.96$ (ddt, $J=16.4,10.9,5.9,2 \mathrm{H}$, $\left.\mathrm{CH}=\mathrm{CH}_{2}\right), 6.85(\mathrm{~d}, J=2.2,2 \mathrm{H}, \mathrm{IM}-\mathrm{CH}), 7.29-7.35$ (overlapping resonances, $3 \mathrm{H}, m$ - and $p$-pyr- $\left.\mathrm{CH}\right), 7.78(\mathrm{~d}, J$ $=2.2,2 \mathrm{H}, \mathrm{IM}-\mathrm{CH}) .{ }^{13} \mathrm{C} \mathrm{NMR}\left(\mathrm{C}_{6} \mathrm{D}_{6}, 25{ }^{\circ} \mathrm{C}, 126 \mathrm{MHz}\right) \delta=9.55\left(\mathrm{PCH}_{2} \mathrm{CH}_{3}\right), 26.93\left(\mathrm{t}, J=8.4, \mathrm{PCH}_{2} \mathrm{CH}_{3}\right), 29.27(\mathrm{t}$, $\left.J=18.3, \mathrm{PCH}_{2} \mathrm{CH}_{2} \mathrm{P}\right), 54.05\left(\mathrm{~N}-\mathrm{CH}_{2}\right), 96.36,107.71,111.38,118.51,120.42,135.49,140.08$ (p-pyr- $\left.\mathrm{CH}\right)$, $211.52\left(\mathrm{t}, J=16.2\right.$, carbene-C). ${ }^{31} \mathrm{P} \mathrm{NMR}\left(\mathrm{C}_{6} \mathrm{D}_{6}, 25^{\circ} \mathrm{C}, 202 \mathrm{MHz}\right) \delta=106.78$ (depe). UV-Vis (THF, ambient temperature; $\lambda_{\max }$, molar absorptivity): $363 \mathrm{~nm}, 11100 \mathrm{M}^{-1} \mathrm{~cm}^{-1} ; 441 \mathrm{~nm}, 18500 \mathrm{M}^{-1} \mathrm{~cm}^{-1}$. Selected IR data $(\mathrm{KBr}) \mathrm{v}=1478,1407,1257,1221,1164,1061,996,716 \mathrm{~cm}^{-1}$.

Synthesis of (allyl $\mathrm{CNC}) \mathrm{Fe}\left(\mathrm{CN}\left(2,4,6-{ }^{t} \mathrm{Bu}_{3} \mathrm{C}_{6} \mathrm{H}_{2}\right)\right)_{2}\left(4-{ }^{t} \mathrm{Bu}_{3} \mathrm{C}_{6} \mathrm{H}_{2}\right)$. A $20 \mathrm{~mL}$ scintillation vial was charged with $\left[\left({ }^{\text {allyl }} \mathrm{CNC}\right) \mathrm{Fe}(\mathrm{MeCN})_{3}\right]\left[\mathrm{BPh}_{4}\right]_{2}$ (2) (0.472 $\left.\mathrm{g}, 0.427 \mathrm{mmol}\right), 2,4,6$-tri-tert-butylphenylisocyanide $(0.231 \mathrm{~g}, 0.851$ $\mathrm{mmol})$, and $12 \mathrm{~mL}$ THF and frozen. Upon thawing, potassium graphite $(0.118 \mathrm{~g}, 0.873 \mathrm{mmol})$ was added resulting in a rapid color change to deep brown. The mixture was stirred for $20 \mathrm{~min}$ and volatiles were removed in vacuo. The product was extracted into $20 \mathrm{~mL}$ diethyl ether, filtered, and dried to afford black powder (0.344 g, $0.386 \mathrm{mmol}, 91 \%)$ assigned as (allyl $\mathrm{CNC}) \mathrm{Fe}\left(\mathrm{CN}\left(2,4,6-{ }^{\mathrm{t}} \mathrm{Bu}_{3} \mathrm{C}_{6} \mathrm{H}_{2}\right)\right)_{2}\left({ }^{4}-{ }^{t} \mathrm{Bu}_{3} \mathrm{C}_{6} \mathrm{H}_{2}\right)$. Single, X-ray quality crystals were obtained by slow evaporation of a $n$-pentane solution of (allyl $\mathrm{CNC}) \mathrm{Fe}(\mathrm{CN}(2,4,6$ $\left.\left.{ }^{t} \mathrm{Bu}_{3} \mathrm{C}_{6} \mathrm{H}_{2}\right)\right)_{2}\left({ }_{4-}{ }^{t} \mathrm{Bu}_{3} \mathrm{C}_{6} \mathrm{H}_{2}\right)$ stored at $-35{ }^{\circ} \mathrm{C}$. MALDI-TOF for $[\mathrm{M}]^{+}\left(\mathrm{C}_{55} \mathrm{H}_{75} \mathrm{~N}_{7} \mathrm{Fe}_{1}\right)$ : Calc. 889.543; Found 889.892. ${ }^{1} \mathrm{H}$ NMR $\left(\mathrm{C}_{6} \mathrm{D}_{6}, 25{ }^{\circ} \mathrm{C}, 500 \mathrm{MHz}\right) \delta=1.22\left(\mathrm{~s}, 18 \mathrm{H}, \mathrm{Ph}-\mathrm{CH}_{3}\right), 1.37\left(\mathrm{~s}, 36 \mathrm{H}, \mathrm{Ph}-\mathrm{CH}_{3}\right), 4.82(\mathrm{~d}, \mathrm{~J}=10.2,2 \mathrm{H}$, $\mathrm{CH}=\mathrm{CHH}), 5.01(\mathrm{dd}, J=17.4,2.1,2 \mathrm{H}, \mathrm{CH}=\mathrm{CHH}), 5.33\left(\mathrm{~d}, J=5.9,4 \mathrm{H}, \mathrm{N}-\mathrm{CH}_{2}\right), 6.02(\mathrm{ddt}, J=16.4,10.9,5.9$, $\left.2 \mathrm{H}, \mathrm{CH}=\mathrm{CH}_{2}\right), 6.75(\mathrm{~d}, \mathrm{~J}=2.2,2 \mathrm{H}, \mathrm{IM}-\mathrm{CH}), 7.22$ (overlapping resonances, $3 \mathrm{H}, m$ - and $p$-pyr- $\left.\mathrm{CH}\right), 7.36(\mathrm{~s}, 4 \mathrm{H}$,

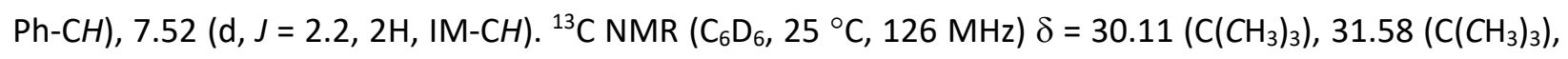

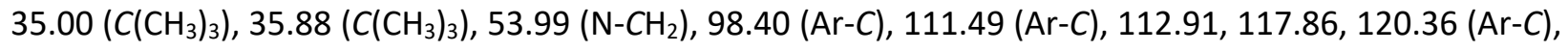
121.51 (Ar-C), 129.19 (Ar-C), 135.59, 142.77, 145.07 (Ar-C), 145.78 (Ar-C), $193.63(C \equiv N-), 211.26$ (carbeneC). UV-Vis (THF, ambient temperature; $\lambda_{\max }$, molar absorptivity): $272 \mathrm{~nm}, 12900 \mathrm{M}^{-1} \mathrm{~cm}^{-1} ; 392 \mathrm{~nm}, 9800 \mathrm{M}^{-}$ ${ }^{1} \mathrm{~cm}^{-1} ; 518 \mathrm{~nm}, 2800 \mathrm{M}^{-1} \mathrm{~cm}^{-1}$. Selected IR data $(\mathrm{KBr}) \mathrm{v}=1992,1896 \mathrm{~cm}^{-1}(\mathrm{CN})$. 
Synthesis of (allyl $\mathrm{CNC}) \mathrm{Fe}(\mathrm{CNPh})_{2}$ (4-Ph). A $20 \mathrm{~mL}$ scintillation vial was charged with $\left[\left({ }^{\text {allyl }} \mathrm{CNC}\right) \mathrm{Fe}(\mathrm{MeCN})_{3}\right]\left[\mathrm{BPh}_{4}\right]_{2}(2)(0.990 \mathrm{~g}, 0.895 \mathrm{mmol})$ and $12 \mathrm{~mL}$ THF. While stirring, phenylisocyanide $(0.185 \mathrm{~g}, 1.794 \mathrm{mmol})$ was added and the mixture frozen. Upon thawing, potassium graphite $(0.232 \mathrm{~g}$, $1.716 \mathrm{mmol}$ ) was added resulting in a rapid color change to deep red. The mixture was stirred for $20 \mathrm{~min}$ and volatiles were removed in vacuo. The product was extracted into a mixture of benzene and toluene (40 mL, $20 \mathrm{~mL}$, respectively), filtered, and dried to afford dark red powder $(0.316 \mathrm{~g}, 0.571 \mathrm{mmol}, 64 \%)$ assigned as ( $\left.{ }^{\text {allyl }} \mathrm{CNC}\right) \mathrm{Fe}(\mathrm{CNPh})_{2}$ (4-Ph). Single, X-ray quality crystals were obtained by slow diffusion of $n$ pentane into a toluene solution of (allyl $\mathrm{CNC}) \mathrm{Fe}(\mathrm{CNPh})_{2}$ (4-Ph) at $-35{ }^{\circ} \mathrm{C}$. HR LC-MS of $[\mathrm{M}]+\mathrm{H}\left(\mathrm{C}_{31} \mathrm{H}_{27} \mathrm{~N}_{7} \mathrm{Fe}_{1}+\right.$ $\mathrm{H})$ : calc. 554.1756; found 554.1734. ${ }^{1} \mathrm{H} \mathrm{NMR}\left(\mathrm{C}_{6} \mathrm{D}_{6}, 25^{\circ} \mathrm{C}, 500 \mathrm{MHz}\right) \delta=4.93(\mathrm{dd}, \mathrm{J}=10.2,1.7,2 \mathrm{H}, \mathrm{CH}=\mathrm{CHH})$, $5.05(\mathrm{dd}, J=17.2,1.8,2 \mathrm{H}, \mathrm{CH}=\mathrm{CHH}), 5.24\left(\mathrm{~d}, J=6.1,4 \mathrm{H}, \mathrm{N}-\mathrm{CH}_{2}\right), 6.16$ (ddt, $J=16.4,10.1,6.0,2 \mathrm{H}, \mathrm{CH}=\mathrm{CH}_{2}$ ), $6.53(\mathrm{~d}, J=2.2,2 \mathrm{H}, \mathrm{IM}-\mathrm{CH}), 6.78(\mathrm{t}, J=7.4,2 \mathrm{H}, p-\mathrm{Ph}-\mathrm{CH}), 6.96(\mathrm{t}, J=7.9,4 \mathrm{H}, m-\mathrm{Ph}-\mathrm{CH}), 6.97(\mathrm{~d}, J=7.6,2 \mathrm{H}$, $m$-pyr-CH), 7.13 (t, $J=7.6,1 \mathrm{H} \mathrm{p}$-pyr-CH), 7.22 (d, J = 7.9, 4H, o-Ph-CH), 7.26 (d, J=2.2, 2H, IM-CH). ${ }^{13} \mathrm{C} \mathrm{NMR}$ $\left(\mathrm{C}_{6} \mathrm{D}_{6}, 25^{\circ} \mathrm{C}, 126 \mathrm{MHz}\right) \delta=53.12\left(\mathrm{~N}-\mathrm{CH}_{2}\right), 99.26(\mathrm{Ar}-\mathrm{C}), 112.29,117.56,117.92,121.23,123.55,124.65$, $129.39,135.15,138.89,143.81,213.17$ ( $C \equiv \mathrm{N}$-Ph or carbene- $C$ ), 213.53 ( $C \equiv \mathrm{N}-\mathrm{Ph}$ or carbene- $C$ ). UV-Vis (THF, ambient temperature; $\lambda_{\max }$, molar absorptivity): $279 \mathrm{~nm}, 7300 \mathrm{M}^{-1} \mathrm{~cm}^{-1} ; 366 \mathrm{~nm}, 5300 \mathrm{M}^{-1} \mathrm{~cm}^{-1} ; 541 \mathrm{~nm}$, $3300 \mathrm{M}^{-1} \mathrm{~cm}^{-1}$. Selected IR data $(\mathrm{KBr}) \mathrm{v}=1951,1735 \mathrm{~cm}^{-1}(\mathrm{CN})$.

Synthesis of $\quad\left({ }^{\text {allyl }} \mathrm{CNC}\right) \mathrm{Fe}\left(\mathrm{CNN}^{t} \mathrm{Bu}\right)_{2} \quad\left(\mathbf{4}^{t}{ }^{t} \mathrm{Bu}\right) . \quad$ A $20 \mathrm{~mL}$ scintillation vial was charged with $\left[\left({ }^{\text {allyl }} \mathrm{CNC}\right) \mathrm{Fe}(\mathrm{MeCN})_{3}\right]\left[\mathrm{BPh}_{4}\right]_{2}$ (2) $(0.298 \mathrm{~g}, 0.269 \mathrm{mmol})$ and $10 \mathrm{~mL}$ THF. While stirring, tert-butylisocyanide $(0.060 \mathrm{~mL}, 0.530 \mathrm{mmol})$ was added resulting in a slight darkening of the color or the slurry. The mixture was frozen. Upon thawing, potassium graphite $(0.072 \mathrm{~g}, 0.533 \mathrm{mmol})$ was added resulting in a rapid color change to dark brown. The mixture was stirred for $20 \mathrm{~min}$ and volatiles were removed in vacuo. The product was extracted into diethyl ether $(15 \mathrm{~mL})$, filtered over Celite, and dried to afford dark brown

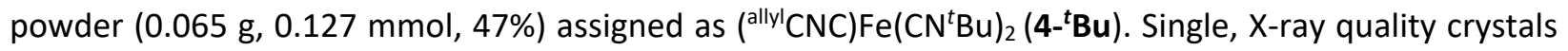
were obtained by layering a benzene solution of $\left({ }^{\text {allyl }} \mathrm{CNC}\right) \mathrm{Fe}\left(\mathrm{CN}^{t} \mathrm{Bu}\right)_{2}\left(4^{-}{ }^{t} \mathrm{Bu}\right)$ with hexamethyldisiloxane at room temperature. HR LC-MS of [M]+H $\left(\mathrm{C}_{27} \mathrm{H}_{35} \mathrm{~N}_{7} \mathrm{Fe}_{1}+\mathrm{H}\right)$ : calc. 514.2382; found 514.3549. MALDI-TOF of $[\mathrm{M}]^{+}\left(\mathrm{C}_{33} \mathrm{H}_{39} \mathrm{~N}_{5} \mathrm{P}_{2} \mathrm{Fe}_{1}\right)$ : calc. 513.2303; found 512.649. ${ }^{1} \mathrm{H}$ NMR $\left(\mathrm{C}_{6} \mathrm{D}_{6}, 25{ }^{\circ} \mathrm{C}, 700 \mathrm{MHz}\right) \delta=1.14(\mathrm{~s}, 18 \mathrm{H}$, $\left.\mathrm{C}\left(\mathrm{CH}_{3}\right)_{3}\right), 5.18(\mathrm{~d}, J=10.2,2 \mathrm{H}, \mathrm{CH}=\mathrm{CHH}), 5.32(\mathrm{~d}, J=17.3,2 \mathrm{H}, \mathrm{CH}=\mathrm{CHH}), 5.49\left(\mathrm{~d}, J=6.2,4 \mathrm{H}, \mathrm{N}-\mathrm{CH}_{2}\right), 6.43$ (ddt, $J=16.6,10.1,6.2,2 \mathrm{H}, \mathrm{CH}=\mathrm{CH}_{2}$ ), $6.78\left(\mathrm{~d}, J=1.8,2 \mathrm{H}\right.$, IM-CH), 7.16 (overlapping with $\mathrm{C}_{6} \mathrm{D}_{6}$ solvent, $2 \mathrm{H}$, $m$-pyr-CH), $7.33\left(\mathrm{t}, J=7.4, p\right.$-pyr-CH), $7.41(\mathrm{~d}, J=1.8,2 \mathrm{H}, \mathrm{IM}-\mathrm{CH}) .{ }^{13} \mathrm{C} \mathrm{NMR}\left(\mathrm{C}_{6} \mathrm{D}_{6}, 25^{\circ} \mathrm{C}, 126 \mathrm{MHz}\right) \delta=31.67$

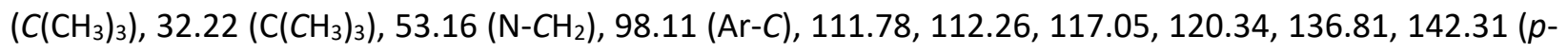
pyr- $\mathrm{CH}$ ), $202.48\left(\mathrm{CN}^{\mathrm{t}} \mathrm{Bu}\right.$ ), 213.88 (carbene- $C$ ). UV-Vis (THF, ambient temperature; $\lambda_{\max }$, molar absorptivity): $408 \mathrm{~nm}, 4200 \mathrm{M}^{-1} \mathrm{~cm}^{-1} ; 523 \mathrm{~nm}, 2900 \mathrm{M}^{-1} \mathrm{~cm}^{-1}$. Selected IR data $(\mathrm{KBr}) \mathrm{v}=2973,1813$ (very strong, broad, CN), 1483, 1410, 1255, 1197, 749, 704, 666, $439 \mathrm{~cm}^{-1}$. 
Generation of ( $\left.{ }^{\mathrm{BBN} C N C}\right) \mathrm{Fe}(\mathrm{CNPh})_{2}$ (5-Ph). A $20 \mathrm{~mL}$ scintillation vial was charged with (allyl $\left.\mathrm{CNC}\right) \mathrm{Fe}(\mathrm{CNPh})_{2}$ (4-Ph) $(0.120 \mathrm{~g}, 0.217 \mathrm{mmol})$ and $6 \mathrm{~mL}$ THF. While stirring, 9-borabicyclo[3.3.1]nonane $(0.060 \mathrm{~g}, 0.492$ mmol) was added and that mixture stirred $24 \mathrm{hr}$. Investigation of an aliquot by ${ }^{1} \mathrm{H}$ NMR spectroscopy revealed full conversion of starting material to a new species, assigned as ( $\left.{ }^{\mathrm{BBN} C N C}\right) \mathrm{Fe}(\mathrm{CNPh})_{2}$ (5-Ph). Volatiles were removed in vacuo and the resulting material washed with $2 \times 10 \mathrm{~mL} n$-pentane and dried to afford dark red powder $(0.159 \mathrm{~g})$ assigned as $\left({ }^{\mathrm{BBN} C N C}\right) \mathrm{Fe}(\mathrm{CNPh})_{2}$ (5-Ph). This material is not spectroscopically pure; however, it was used for the spectroscopic characterization data provided below. Therefore, assignments may be incomplete. MALDI-TOF for [M]+H $\left(\mathrm{C}_{47} \mathrm{H}_{57} \mathrm{~N}_{7} \mathrm{~B}_{2} \mathrm{Fe}_{1}+\mathrm{H}\right)$ : Calc. 798.4289; Found 798.632. ${ }^{1} \mathrm{H}$ NMR $\left(\mathrm{C}_{6} \mathrm{D}_{6}, 25{ }^{\circ} \mathrm{C}, 500 \mathrm{MHz}\right) \delta=0.29(\mathrm{~s}, 2 \mathrm{H}, \mathrm{B}-\mathrm{CH}), 1.16(\mathrm{~m}, \mathrm{BBN}-\mathrm{CH}), 1.41(\mathrm{t}, J=7.9$, $\left.4 \mathrm{H}, \mathrm{B}-\mathrm{CH}_{2}\right), 1.57-1.67(\mathrm{~m}, 9-\mathrm{BBN}-\mathrm{CH}), 1.73-1.86(\mathrm{~m}, \mathrm{BBN}-\mathrm{CH}), 2.19\left(\mathrm{p}, J=8.0,4 \mathrm{H}, \mathrm{CH}_{2} \mathrm{CH}_{2} \mathrm{CH}_{2}\right), 4.68(\mathrm{t}, J=$ 7.7, 4H, N-CH $)_{2}, 6.59(\mathrm{~s}, 2 \mathrm{H}, \mathrm{IM}-\mathrm{CH}), 6.79(\mathrm{t}, J=7.4,2 \mathrm{H}, p$-phenyl- $\mathrm{CH}), 7.01$ (t, J = 7.8, 4H, m-Ph-CH), 7.32 $(\mathrm{d}, J=7.4,4 \mathrm{H}, o-\mathrm{Ph}-\mathrm{CH}) .{ }^{13} \mathrm{C}$ NMR $\left(\mathrm{C}_{6} \mathrm{D}_{6}, 25{ }^{\circ} \mathrm{C}, 126 \mathrm{MHz}\right) \delta=23.67,25.52,26.63,31.26,33.40\left(\mathrm{BBN}-\mathrm{CH}_{2}\right)$, $53.15\left(\mathrm{~N}-\mathrm{CH}_{2}\right), 99.43(\mathrm{Ar}-\mathrm{C}), 112.23,118.51,121.55,123.57,124.62,129.37,139.59,138.89,144.14$, $212.56(C \equiv \mathrm{N}-\mathrm{Ph}$ or carbene- $C), 217.19(C \equiv \mathrm{N}-\mathrm{Ph}$ or carbene- $C)$. Selected IR data $(\mathrm{KBr}) \mathrm{v}=1988,1735 \mathrm{~cm}^{-1}$ (CN). A strong band at $2041 \mathrm{~cm}^{-1}$ arises from the decomposition of this material (see spectra below). Decomposition of $\left({ }^{\mathrm{BBN}} \mathrm{CNC}\right) \mathrm{Fe}(\mathrm{CNPh})_{2}$ (5-Ph): A portion of $\left({ }^{\mathrm{BBN}} \mathrm{CNC}\right) \mathrm{Fe}(\mathrm{CNPh})_{2}$ (5-Ph) was dissolved in toluene to afford a deep red/purple solution. After sitting overnight (ca. $16 \mathrm{hr}$ ), an olive-green powder precipitated. This material was investigated by NMR (THF) and IR ( $\mathrm{KBr}$ ) spectroscopies. The NMR revealed only broad, ill-defined resonances in the diamagnetic region. No paramagnetically shifted resonances were observed. The spectrum is provided below. The infrared spectrum reveals a dominant band at 2052 $\mathrm{cm}^{-1}$. This band is a minor component in isolated samples of $\left({ }^{\mathrm{BBN}} \mathrm{CNC}\right) \mathrm{Fe}(\mathrm{CNPh})_{2}(5-\mathrm{Ph})$.

Synthesis of $\left({ }^{\mathrm{BBN}} \mathrm{CNC}\right) \mathrm{Fe}\left(\mathrm{CN}\left(2,4,6-{ }^{t} \mathrm{Bu}_{3} \mathrm{C}_{6} \mathrm{H}_{2}\right)\right)_{2}\left(5-{ }^{\mathrm{B}} \mathrm{Bu}_{3} \mathrm{C}_{6} \mathrm{H}_{2}\right)$. A $20 \mathrm{~mL}$ scintillation vial was charged with $\left({ }^{\text {allyl }} \mathrm{CNC}\right) \mathrm{Fe}\left(\mathrm{CN}\left(2,4,6-{ }^{t} \mathrm{Bu}_{3} \mathrm{C}_{6} \mathrm{H}_{2}\right)\right)_{2}\left({ }^{4}-{ }^{t} \mathrm{Bu}_{3} \mathrm{C}_{6} \mathrm{H}_{2}\right)(0.021 \mathrm{~g}, 0.024 \mathrm{mmol})$ and $0.8 \mathrm{~mL} \mathrm{C} \mathrm{C}_{6}$. To this vial, 9borabicyclo[3.3.1]nonane $(0.007 \mathrm{~g}, 0.057 \mathrm{mmol})$ was added and the solution transferred to an NMR tube. After $4 \mathrm{hr}$ at room temperature, ${ }^{1} \mathrm{H}$ NMR spectroscopy revealed clean generation of a new species assigned as $\left({ }^{\mathrm{BBN}} \mathrm{CNC}\right) \mathrm{Fe}\left(\mathrm{CN}\left(2,4,6-{ }_{-}{ }^{\mathrm{B}} \mathrm{Bu}_{3} \mathrm{C}_{6} \mathrm{H}_{2}\right)\right)_{2} \quad\left(5_{-}{ }^{\mathrm{t}} \mathrm{Bu}_{3} \mathrm{C}_{6} \mathrm{H}_{2}\right)$. This material contains residual 9borabicyclo[3.3.1]nonane. The compound is stable to a large excess ( $>10$ fold $)$ of 9borabicyclo[3.3.1]nonane (see spectrum below). The material from the NMR tube was transferred to a 20 $\mathrm{mL}$ scintillation vial and volatiles were removed in vacuo. Dissolution of the material in minimal $n$-pentane $(\sim 1 \mathrm{~mL})$ followed by slow evaporation at $-35{ }^{\circ} \mathrm{C}$ afforded single, X-ray quality crystals. HR LC-MS of [M]+H $\left(\mathrm{C}_{71} \mathrm{H}_{105} \mathrm{~N}_{7} \mathrm{~B}_{2} \mathrm{Fe}_{1}+\mathrm{H}\right)$ : calc. 1134.8045 ; found 1134.8052. MALDI-TOF of $[\mathrm{M}]^{+}\left(\mathrm{C}_{71} \mathrm{H}_{105} \mathrm{~N}_{7} \mathrm{~B}_{2} \mathrm{Fe}_{1}\right)$ : calc. 1133.7967; found 1133.808. ${ }^{1} \mathrm{H} \mathrm{NMR}\left(\mathrm{C}_{6} \mathrm{D}_{6}, 25^{\circ} \mathrm{C}, 400 \mathrm{MHz}\right) \delta=1.08(\mathrm{~m}, 4 \mathrm{H}), 1.26\left(\mathrm{~s}, 18 \mathrm{H}, p-\mathrm{C}\left(\mathrm{CH}_{3}\right)_{3}\right), 1.36$ $\left(\mathrm{s}, 36 \mathrm{H}, \mathrm{o}-\mathrm{C}\left(\mathrm{CH}_{3}\right)_{3}\right), 1.39(\mathrm{~m}, 4 \mathrm{H}), 1.51(\mathrm{~m}, 12 \mathrm{H}(8 \mathrm{H}+4 \mathrm{H}$ overlap)), $1.74(\mathrm{~m}, 8 \mathrm{H}), 1.87(\mathrm{~m}, 4 \mathrm{H}), 2.09(\mathrm{p}, \mathrm{J}=$ $\left.7.8,4 \mathrm{H}, \mathrm{CH}_{2} \mathrm{CH}_{2} \mathrm{CH}_{2}\right), 4.78\left(\mathrm{t}, J=7.1,4 \mathrm{H}, \mathrm{N}-\mathrm{CH}_{2}\right), 6.81(\mathrm{~d}, J=1.8,2 \mathrm{H}, \mathrm{IM}-\mathrm{CH}), 7.27(\mathrm{~m}, 3 \mathrm{H}, m$ - and $p-p y r-\mathrm{CH}$, overlap), $7.30(\mathrm{~s}, 4 \mathrm{H}, \mathrm{m}-\mathrm{Ar}-\mathrm{CH}), 7.60(\mathrm{~d}, J=1.8,2 \mathrm{H}, \mathrm{IM}-\mathrm{CH}) .{ }^{11} \mathrm{~B} \mathrm{NMR}\left(\mathrm{C}_{6} \mathrm{D}_{6}, 25{ }^{\circ} \mathrm{C}, 128 \mathrm{MHz}\right) \delta=88.07$. The reaction was repeated with twelve equiv. 9-borabicyclo[3.3.1]nonane. The ${ }^{1} \mathrm{H}$ NMR spectrum is identical to that obtained with 2.2 equiv. 9-borabicyclo[3.3.1]nonane suggesting the material is stable to excess 9 borabicyclo[3.3.1]nonane. 
Synthesis of ( $\left.{ }^{\mathrm{BPin}} \mathrm{CNC}\right) \mathrm{Fe}\left(\mathrm{PMe}_{3}\right)_{2}$ (6-PMe $)_{3}$. A $20 \mathrm{~mL}$ scintillation vial was charged with $0.107 \mathrm{~g}(0.078 \mathrm{mmol})$ $\left[\left({ }^{B P i n} \mathrm{CNC}\right) \mathrm{Fe}(\mathrm{MeCN})_{3}\right]\left[\mathrm{BPh}_{4}\right]_{2}$, trimethylphosphine $(0.016 \mathrm{~mL}, 0.155 \mathrm{mmol})$, and $10 \mathrm{~mL} \mathrm{THF}$ and frozen. On thawing, potassium graphite $(0.022 \mathrm{~g}, 0.163 \mathrm{mmol})$ was added and the solution stirred for $15 \mathrm{~min}$. Volatiles were removed in vacuo and the product extracted into $6 \mathrm{~mL}$ diethyl ether, filtered, and dried to afford black powder $(0.040 \mathrm{~g}, 0.053 \mathrm{mmol}, 68 \%)$ assigned as $\left({ }^{\mathrm{BPin}} \mathrm{CNC}\right) \mathrm{Fe}\left(\mathrm{PMe}_{3}\right)_{2}\left(6-\mathrm{PMe}_{3}\right)$. Single, X-ray quality crystals were obtained by slow evaporation of a diethyl ether solution at $-35^{\circ} \mathrm{C}$. HR LC-MS of [M]+H $\left(\mathrm{C}_{35} \mathrm{H}_{61} \mathrm{~N}_{5} \mathrm{O}_{4} \mathrm{~B}_{2} \mathrm{P}_{2} \mathrm{Fe}_{1}+\mathrm{H}\right)$ : calc. 756.3813; found 756.3816. ${ }^{1} \mathrm{H} N M R\left(\mathrm{C}_{6} \mathrm{D}_{6}, 25{ }^{\circ} \mathrm{C}, 700 \mathrm{MHz}\right) \delta=0.97(\mathrm{~s}, 18 \mathrm{H}$, $\left.\mathrm{P}\left(\mathrm{CH}_{3}\right)_{3}\right), 1.11\left(\mathrm{~s}, 24 \mathrm{H}, \mathrm{BPin}-\mathrm{CH}_{3}\right), 1.19\left(\mathrm{t}, \mathrm{J}=9.0,4 \mathrm{H}, \mathrm{B}-\mathrm{CH}_{2}\right), 2.31\left(\mathrm{~m}, 4 \mathrm{H},-\mathrm{CH}_{2} \mathrm{CH}_{2} \mathrm{CH}_{2}-\right), 4.56(\mathrm{~m}, 4 \mathrm{H}, \mathrm{N}-$ $\left.\mathrm{CH}_{2}\right), 6.95(\mathrm{~d}, J=2.4,2 \mathrm{H}, \mathrm{IM}-\mathrm{CH}), 7.31(\mathrm{~m}, 3 \mathrm{H}, \mathrm{m}$ - and $p$-pyr- $\mathrm{CH}), 7.74(\mathrm{~d}, J=2.4,2 \mathrm{H}, \mathrm{IM}-\mathrm{CH}) .{ }^{13} \mathrm{C} \mathrm{NMR}\left(\mathrm{C}_{6} \mathrm{D}_{6}\right.$, $\left.25^{\circ} \mathrm{C}, 126 \mathrm{MHz}\right) \delta=9.67\left(-\mathrm{CH}_{2} \mathrm{BPin}\right), 25.00\left(-\mathrm{CH}_{2} \mathrm{CH}_{2} \mathrm{CH}_{2}-\right), 25.04\left(\mathrm{BPin}-\mathrm{CH}_{3}\right), 27.45\left(\mathrm{t}, \mathrm{J}=7.6, \mathrm{P}\left(\mathrm{CH}_{3}\right)_{3}\right), 52.93$ $\left(\mathrm{N}-\mathrm{CH}_{2}\right), 83.21$ (BPin- $\left.\mathrm{C}\left(\mathrm{CH}_{3}\right)_{2}\right), 96.76(\mathrm{Ar}-\mathrm{C}), 106.24$ (Ar-C), 110.74 (Ar-C), 119.08 (Ar-C), 141.74 (p-pyr-CH), $214.23(\mathrm{t}, J=18.9$, carbene- $C) .{ }^{31} \mathrm{P}$ NMR $\left(\mathrm{C}_{6} \mathrm{D}_{6}, 25^{\circ} \mathrm{C}, 202 \mathrm{MHz}\right) \delta=13.02\left(\mathrm{P}\left(\mathrm{CH}_{3}\right)_{3}\right) .{ }^{11} \mathrm{~B} \mathrm{NMR}\left(\mathrm{C}_{6} \mathrm{D}_{6}, 25{ }^{\circ} \mathrm{C}\right.$, $225 \mathrm{MHz}$ ) $\delta=34.21$. UV-Vis (THF, ambient temperature; $\lambda_{\max }$, molar absorptivity): $346 \mathrm{~nm}, 8100 \mathrm{M}^{-1} \mathrm{~cm}^{-1}$; $432 \mathrm{~nm}, 9900 \mathrm{M}^{-1} \mathrm{~cm}^{-1}$. UV-Vis (cyclohexane, ambient temperature; $\left.\lambda_{\max }\right) 349 \mathrm{~nm}, 434 \mathrm{~nm}$. UV-Vis (benzene, ambient temperature; $\left.\lambda_{\max }\right) 352 \mathrm{~nm}, 436 \mathrm{~nm}$. Selected IR data $(\mathrm{KBr}) v=2975,2890,1468,1408$, $1382,1329,1252,1227,1142,926 \mathrm{~cm}^{-1}$.

Synthesis of $\quad\left({ }^{\mathrm{BPin}} \mathrm{CNC}\right) \mathrm{Fe}(\mathrm{CNPh})_{2} \quad$ (6-CNPh). A $20 \mathrm{~mL}$ scintillation vial was charged with $\left[\left({ }^{B P i n} \mathrm{CNC}\right) \mathrm{Fe}(\mathrm{MeCN})_{3}\right]\left[\mathrm{BPh}_{4}\right]_{2}(0.230 \mathrm{~g}, 0.169 \mathrm{mmol})$ and $8 \mathrm{~mL}$ THF. While stirring, phenylisocyanide $(0.035$ g, $0.339 \mathrm{mmol}$ ) was added resulting in a color change to deep orange/red. The mixture was frozen. Upon thawing, potassium graphite $(0.045 \mathrm{~g}, 0.333 \mathrm{mmol})$ was added resulting in a rapid color change to dark brown/red. The mixture was stirred for $20 \mathrm{~min}$ and volatiles were removed in vacuo. The product was extracted into diethyl ether $(15 \mathrm{~mL})$, filtered over Celite, and dried to afford dark red/brown powder $(0.075 \mathrm{~g}, 0.093 \mathrm{mmol}, 55 \%)$ assigned as ( $\left.{ }^{\mathrm{BPin}} \mathrm{CNC}\right) \mathrm{Fe}(\mathrm{CNPh})_{2}$ (6-CNPh). HR LC-MS of [M]+H $\left(\mathrm{C}_{43} \mathrm{H}_{53} \mathrm{~N}_{7} \mathrm{O}_{4} \mathrm{~B}_{2} \mathrm{Fe}_{1}\right.$ $+\mathrm{H})$ : calc. 810.3773; found 810.3773. HR LC-MS of [M]-CNPh $\left(\mathrm{C}_{43} \mathrm{H}_{53} \mathrm{~N}_{7} \mathrm{O}_{4} \mathrm{~B}_{2} \mathrm{Fe}_{1}-\mathrm{C}_{7} \mathrm{H}_{5} \mathrm{~N}\right)$ : calc. 706.3273; found 706.3289. ${ }^{1} \mathrm{H} \mathrm{NMR}\left(\mathrm{C}_{6} \mathrm{D}_{6}, 25{ }^{\circ} \mathrm{C}, 700 \mathrm{MHz}\right) \delta=1.02\left(\mathrm{~s}, 18 \mathrm{H}, \mathrm{CH}_{3}\right), 1.05$ (overlapping, $\left.4 \mathrm{H}, \mathrm{B}-\mathrm{CH}_{2}\right), 2.31$ $\left(\mathrm{p}, J=8.0,4 \mathrm{H}, \mathrm{CH}_{2} \mathrm{CH}_{2} \mathrm{CH}_{2}\right), 4.69\left(\mathrm{t}, J=7.8,4 \mathrm{H}, \mathrm{N}-\mathrm{CH}_{2}\right), 6.60(\mathrm{~s}, 2 \mathrm{H}, \mathrm{IM}-\mathrm{CH}), 6.79(\mathrm{t}, J=7.4,2 \mathrm{H}, p-\mathrm{Ph}-\mathrm{CH})$, $6.94(\mathrm{~d}, J=7.6,2 \mathrm{H}, m$-pyr- $\mathrm{CH}), 7.01(\mathrm{t}, J=7.7,4 \mathrm{H}, m-\mathrm{Ph}-\mathrm{CH}), 7.15$ (overlapping, $1 \mathrm{H}, p$-pyr- $\mathrm{CH}$ ), 7.25 (s, $2 \mathrm{H}$, IM-CH), $7.31(\mathrm{~d}, \mathrm{~J}=7.9,4 \mathrm{H}, \mathrm{o}-\mathrm{Ph}-\mathrm{CH}) .{ }^{13} \mathrm{C}$ NMR $\left(\mathrm{C}_{6} \mathrm{D}_{6}, 25{ }^{\circ} \mathrm{C}, 126 \mathrm{MHz}\right) \delta=9.14\left(\mathrm{~B}-\mathrm{CH}_{2}\right), 24.97\left(\mathrm{CH}_{3}\right), 26.04$ $\left(\mathrm{CH}_{2} \mathrm{CH}_{2} \mathrm{CH}_{2}\right), 52.66\left(\mathrm{~N}-\mathrm{CH}_{2}\right), 82.84\left(\mathrm{O}_{2} \mathrm{C}_{2} \mathrm{Me}_{4}\right), 99.07,111.85,117.56,121.60,123.24,124.73,137.55$, 139.37, 143.92, $212.61(C \equiv \mathrm{N}-\mathrm{Ph}$ or carbene- $C), 215.31$ ( $C \equiv \mathrm{N}-\mathrm{Ph}$ or carbene- $C) .{ }^{11} \mathrm{~B}$ NMR $\left(\mathrm{C}_{6} \mathrm{D}_{6}, 25{ }^{\circ} \mathrm{C}, 128\right.$ $\mathrm{MHz}) \delta=34.10$. UV-Vis (THF, ambient temperature; $\lambda_{\max }$, molar absorptivity): $281 \mathrm{~nm}, 6600 \mathrm{M}^{-1} \mathrm{~cm}^{-1} ; 367$ $\mathrm{nm}, 4400 \mathrm{M}^{-1} \mathrm{~cm}^{-1} ; 546 \mathrm{~nm}, 3100 \mathrm{M}^{-1} \mathrm{~cm}^{-1}$. Selected IR data (KBr) $v=1894$ (broad, very strong), 1713 (broad, very strong) $\mathrm{cm}^{-1}(\mathrm{CN})$. 
Synthesis of $\left({ }^{B P i n} \mathrm{CNC}\right) \mathrm{Fe}\left(\mathrm{CN}\left(2,4,6-{ }^{t} \mathrm{Bu}_{3} \mathrm{C}_{6} \mathrm{H}_{2}\right)\right)_{2}\left(6-\mathrm{CN}^{t} \mathrm{Bu}_{3} \mathrm{C}_{6} \mathrm{H}_{2}\right)$. A $20 \mathrm{~mL}$ scintillation vial was charged with $\left[\left({ }^{\mathrm{BPin}} \mathrm{CNC}\right) \mathrm{Fe}(\mathrm{MeCN})_{3}\right]\left[\mathrm{BPh}_{4}\right]_{2}(0.167 \mathrm{~g}, 0.122 \mathrm{mmol}), 2,4,6$-tri-tert-butylphenylisocyanide $(0.066 \mathrm{~g}, 0.243$ $\mathrm{mmol})$, and $10 \mathrm{~mL}$ THF and frozen. Upon thawing, potassium graphite $(0.034 \mathrm{~g}, 0.252 \mathrm{mmol}$ ) was added resulting in a rapid color change to deep brown. The mixture was stirred for $15 \mathrm{~min}$ and volatiles were removed in vacuo. The product was extracted into $12 \mathrm{~mL}$ diethyl ether, filtered, and dried to afford black powder (0.132 g, $0.115 \mathrm{mmol}, 94 \%)$ assigned as $\left({ }^{\mathrm{BPin}} \mathrm{CNC}\right) \mathrm{Fe}\left(\mathrm{CN}\left(2,4,6-{ }^{t} \mathrm{Bu}_{3} \mathrm{C}_{6} \mathrm{H}_{2}\right)\right)_{2}\left(\mathbf{6}-\mathrm{CN}^{t} \mathrm{Bu}_{3} \mathrm{C}_{6} \mathrm{H}_{2}\right)$. Single, $\mathrm{X}-$ ray quality crystals were obtained from a concentrated $n$-pentane/hexamethyldisiloxane $(5: 1)$ solution stored at $-35{ }^{\circ} \mathrm{C}$. HR LC-MS of $[\mathrm{M}]^{+}\left(\mathrm{C}_{67} \mathrm{H}_{101} \mathrm{~N}_{7} \mathrm{O}_{4} \mathrm{~B}_{2} \mathrm{Fe}_{1}\right)$ : calc. 1145.7451; found 1145.7451. ${ }^{1} \mathrm{H}$ NMR $\left(\mathrm{C}_{6} \mathrm{D}_{6}\right.$, $\left.25{ }^{\circ} \mathrm{C}, 500 \mathrm{MHz}\right) \delta=0.79\left(\mathrm{~s}, 24 \mathrm{H}, \mathrm{BPin}-\mathrm{CH}_{3}\right), 1.08\left(\mathrm{~m}, 4 \mathrm{H},-\mathrm{CH}_{2} \mathrm{~B}\right), 1.10\left(\mathrm{~s}, 18 \mathrm{H}, \mathrm{C}\left(\mathrm{CH}_{3}\right)_{3}\right), 1.20(\mathrm{~s}, 36 \mathrm{H}$, $\left.\mathrm{C}\left(\mathrm{CH}_{3}\right)_{3}\right), 2.00\left(\mathrm{~m}, 4 \mathrm{H},-\mathrm{CH}_{2} \mathrm{CH}_{2} \mathrm{CH}_{2}-\right), 4.56\left(\mathrm{~m}, 4 \mathrm{H}, \mathrm{N}-\mathrm{CH}_{2}\right), 6.67(\mathrm{~s}, 2 \mathrm{H}, \mathrm{IM}-\mathrm{CH}), 7.00(\mathrm{~s}, 1 \mathrm{H}, p-\mathrm{pyr}-\mathrm{CH}), 7.04$ (s, 2H, m-pyr-CH), 7.15 (s, 4H, Ar-CH), $7.35(\mathrm{~s}, 2 \mathrm{H}, \mathrm{IM}-\mathrm{CH}) .{ }^{13} \mathrm{C} N M R\left(\mathrm{C}_{6} \mathrm{D}_{6}, 25{ }^{\circ} \mathrm{C}, 126 \mathrm{MHz}\right) \delta=8.97(-$

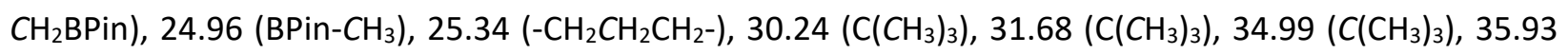
$\left(\mathrm{C}\left(\mathrm{CH}_{3}\right)_{3}\right), 52.48\left(\mathrm{~N}-\mathrm{CH}_{2}\right), 82.80$ (BPin- $\left.\mathrm{C}\left(\mathrm{CH}_{3}\right)_{2}\right), 98.13$ (Ar-C), 110.95 (Ar-C), $120.61(\mathrm{Ar}-\mathrm{C}), 121.37$ (Ar-C), 121.48 (Ar-C), 129.38 (Ar-C), 144.42 (Ar-C), 145.69 (Ar-C), $145.79(\mathrm{Ar}-C), 194.05(C \equiv \mathrm{N}-$ ), 210.27 (carbeneC). ${ }^{11} \mathrm{~B}$ NMR $\left(\mathrm{C}_{6} \mathrm{D}_{6}, 25{ }^{\circ} \mathrm{C}, 128 \mathrm{MHz}\right) \delta=33.70$. UV-Vis (THF, ambient temperature; $\lambda_{\max }$, molar absorptivity): $276 \mathrm{~nm}, 11600 \mathrm{M}^{-1} \mathrm{~cm}^{-1} ; 399 \mathrm{~nm}, 10000 \mathrm{M}^{-1} \mathrm{~cm}^{-1} ; 522 \mathrm{~nm}, 2900 \mathrm{M}^{-1} \mathrm{~cm}^{-1}$. Selected IR data (KBr) $v=1989$, $1895 \mathrm{~cm}^{-1}(\mathrm{CN})$.

Synthesis of ( $\left.{ }^{\mathrm{DPP}} \mathrm{CNC}\right) \mathrm{Fe}\left(\mathrm{PMe}_{3}\right)\left(\mathbf{N}_{2}\right)$. This molecule was synthesized according to a modified literature protocol $^{12}$ for spectroscopic comparison. This reaction was carried out in a $\mathrm{N}_{2}$ filled glovebox. In a $20 \mathrm{~mL}$ scintillation vial, $0.4 \% \mathrm{Na}(\mathrm{Hg})(0.037 \mathrm{~g} \mathrm{Na}, 1.609 \mathrm{mmol})$ was prepared in $5 \mathrm{~mL}$ THF and trimethylphosphine $(0.042 \mathrm{~mL}, 0.406 \mathrm{mmol})$ was added. A separate $20 \mathrm{~mL}$ scintillation vial was charged with ( $\left.{ }^{\mathrm{DIPP}} \mathrm{CNC}\right) \mathrm{FeBr}_{2}{ }^{13}$ $(0.200 \mathrm{~g}, 0.268 \mathrm{mmol})$ and $5 \mathrm{~mL}$ THF. The solution containing ( $\left.{ }^{\mathrm{DIPP}} \mathrm{CNC}\right) \mathrm{FeBr}_{2}$ was added dropwise to the vigorously stirred $\mathrm{Na}(\mathrm{Hg})$ solution. The mixture was stirred vigorously for $3 \mathrm{hr}$ at room temperature. The solution was decanted from the mercury pool and volatiles were removed in vacuo. The resulting material was extracted with $10 \mathrm{~mL}$ diethyl ether, filtered and dried to afford crude ( $\left.{ }^{\mathrm{DIPP}} \mathrm{CNC}\right) \mathrm{Fe}\left(\mathrm{PMe}_{3}\right)\left(\mathrm{N}_{2}\right)$. Purification was achieved by storing a saturated pentane solution of the material at $-35^{\circ} \mathrm{C}$ for 3 days and discarding any precipitates that formed. Removal of volatiles from the mother liquor afforded ( $\left.{ }^{\mathrm{DIPP}} \mathrm{CNC}\right) \mathrm{Fe}\left(\mathrm{PMe}_{3}\right)\left(\mathrm{N}_{2}\right)$. This material was used for spectroscopic analysis. ${ }^{1} \mathrm{H} \mathrm{NMR}\left(\mathrm{C}_{6} \mathrm{D}_{6}, 25{ }^{\circ} \mathrm{C}, 401 \mathrm{MHz}\right)$ $\delta=0.29\left(\mathrm{~d}, J=6.8,9 \mathrm{H}, \mathrm{P}\left(\mathrm{CH}_{3}\right)_{3}\right), 1.00\left(\mathrm{~d}, J=6.9,6 \mathrm{H}, \mathrm{CH}_{3}\right), 1.09\left(\mathrm{~d}, J=6.8,6 \mathrm{H}, \mathrm{CH}_{3}\right), 1.25(\mathrm{~d}, J=6.7,6 \mathrm{H}$, $\left.\mathrm{CH}_{3}\right), 1.36\left(\mathrm{~d}, J=6.8,6 \mathrm{H}, \mathrm{CH}_{3}\right), 3.05$ (heptet, $\left.J=6.8,2 \mathrm{H}, \mathrm{CH}\left(\mathrm{CH}_{3}\right)_{2}\right), 3.50$ (heptet, $\left.J=6.8,2 \mathrm{H}, \mathrm{CH}\left(\mathrm{CH}_{3}\right)_{2}\right), 6.77$ $(\mathrm{d}, J=2.2,2 \mathrm{H}, \mathrm{IM}-\mathrm{CH}), 7.03(\mathrm{~d}, J=7.6,2 \mathrm{H}, m-\mathrm{Ph}-\mathrm{CH}), 7.17-7.28(\mathrm{~m}, 7 \mathrm{H}$, overlapping $p$-pyr- $\mathrm{CH}, m$-pyr- $\mathrm{CH}$, $m$-Ph-CH, $p$-Ph-CH), 7.44 (d, $J=2.2,2 \mathrm{H}, \mathrm{IM}-\mathrm{CH}) .{ }^{31} \mathrm{P} \mathrm{NMR}\left(\mathrm{C}_{6} \mathrm{D}_{6}, 25{ }^{\circ} \mathrm{C}, 162 \mathrm{MHz}\right) \delta=19.40\left(P\left(\mathrm{CH}_{3}\right)_{3}\right) . \mathrm{UV}-$ Vis (THF, ambient temperature; $\lambda_{\max }$, molar absorptivity): $407 \mathrm{~nm}, 14700 \mathrm{M}^{-1} \mathrm{~cm}^{-1} ; 640 \mathrm{~nm}, 3400 \mathrm{M}^{-1} \mathrm{~cm}^{-1}$. Selected IR data $(\mathrm{KBr}) v=2023 \mathrm{~cm}^{-1}\left(\mathrm{~N}_{2}\right)$. 


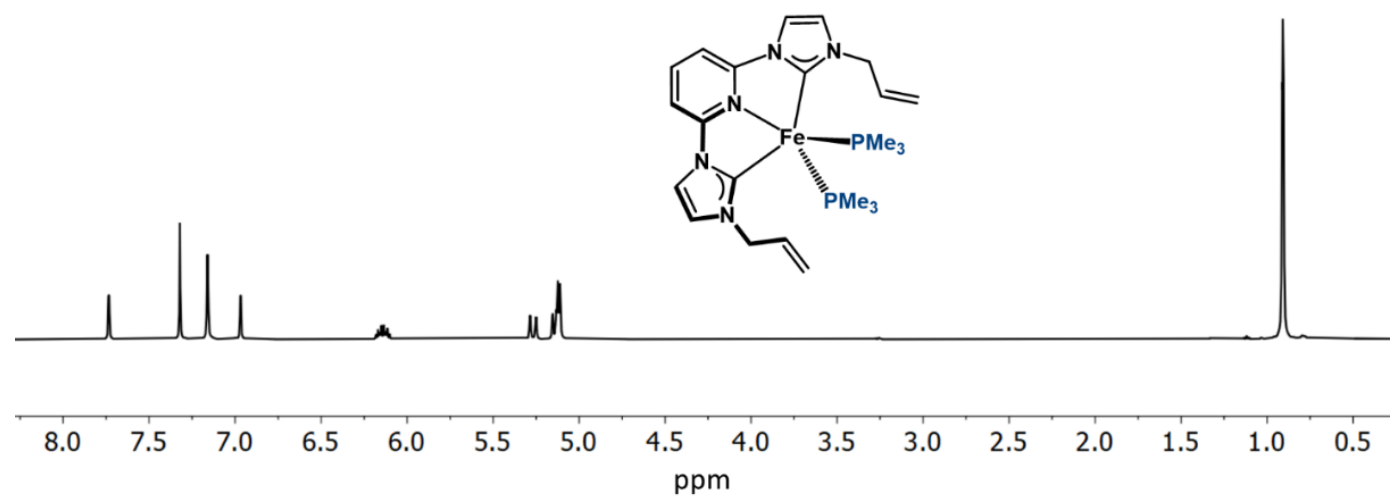

Figure S1 ${ }^{1} \mathrm{H}$ NMR spectrum $\left(\mathrm{C}_{6} \mathrm{D}_{6}, 25^{\circ} \mathrm{C}, 500 \mathrm{MHz}\right)$ of $\left({ }^{\text {allyl }} \mathrm{CNC}\right) \mathrm{Fe}\left(\mathrm{PMe}_{3}\right)_{2}\left(3-\mathrm{PMe}_{3}\right)$.
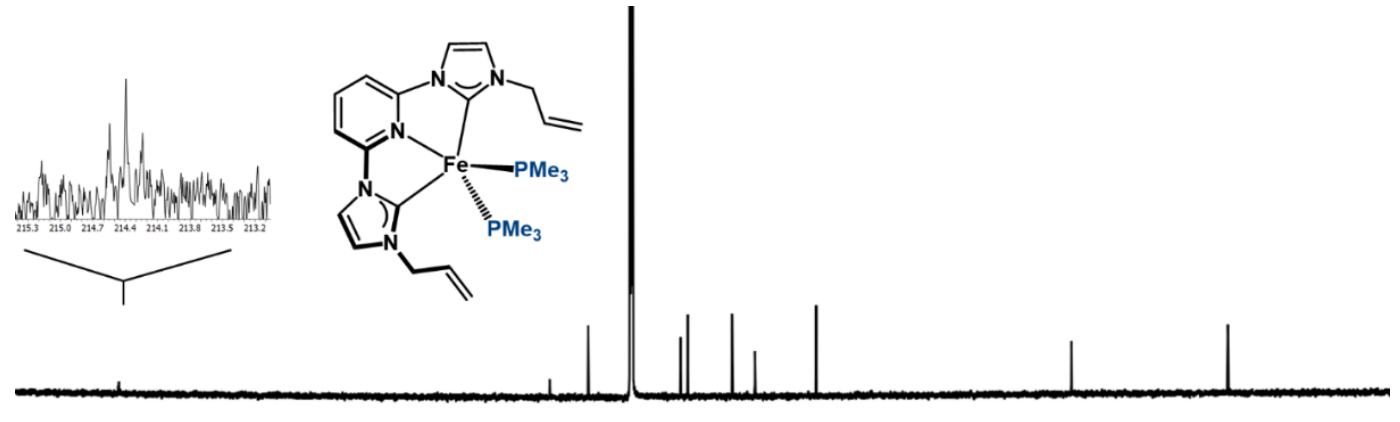

$\begin{array}{lllllllllllllllllllllll}130 & 220 & 210 & 200 & 190 & 180 & 170 & 160 & 150 & 140 & 130 & 120 & 110 & 100 & 90 & 80 & 70 & 60 & 50 & 40 & 30 & 20 & 10\end{array}$ ppm

Figure S2 ${ }^{13} \mathrm{C}$ NMR spectrum $\left(\mathrm{C}_{6} \mathrm{D}_{6}, 25^{\circ} \mathrm{C}, 126 \mathrm{MHz}\right)$ of $\left({ }^{\text {allyl }} \mathrm{CNC}\right) \mathrm{Fe}\left(\mathrm{PMe}_{3}\right)_{2}\left(3-\mathrm{PMe}_{3}\right)$.

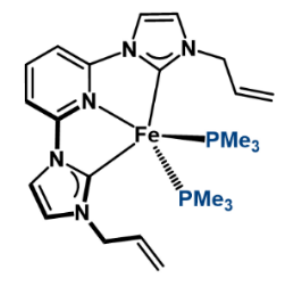

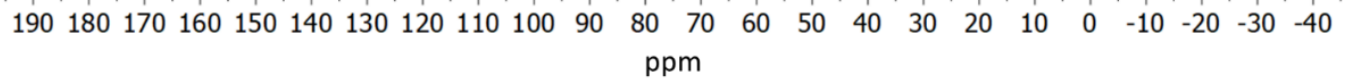

Figure S3 ${ }^{31} \mathrm{P}$ NMR spectrum $\left(\mathrm{C}_{6} \mathrm{D}_{6}, 25{ }^{\circ} \mathrm{C}, 202 \mathrm{MHz}\right)$ of $\left({ }^{\text {allyl }} \mathrm{CNC}\right) \mathrm{Fe}\left(\mathrm{PMe}_{3}\right)_{2}\left(3-\mathrm{PMe}_{3}\right)$. 


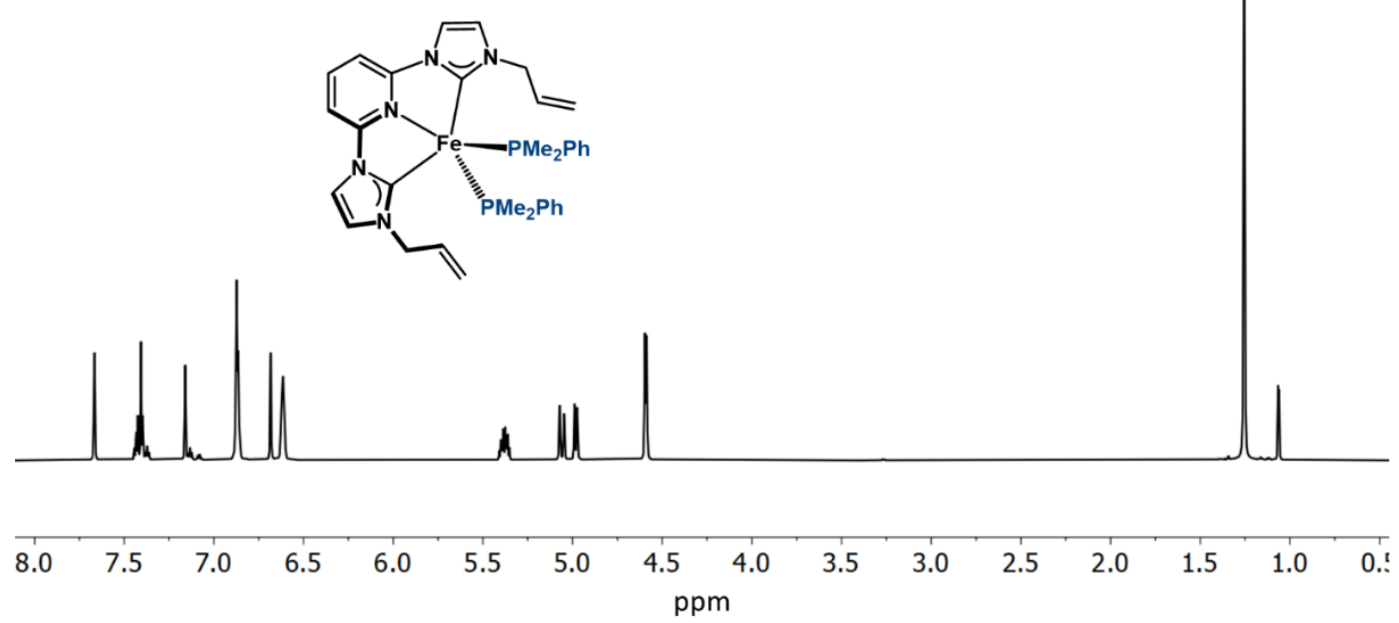

Figure S4 ${ }^{1} \mathrm{H}$ NMR spectrum $\left(\mathrm{C}_{6} \mathrm{D}_{6}, 25{ }^{\circ} \mathrm{C}, 700 \mathrm{MHz}\right)$ of $\left({ }^{\text {allyl } \mathrm{CNC}}\right) \mathrm{Fe}\left(\mathrm{PMe}_{2} \mathrm{Ph}\right)_{2}\left(3-\mathrm{PMe} \mathbf{P}_{2} \mathrm{Ph}\right)$.

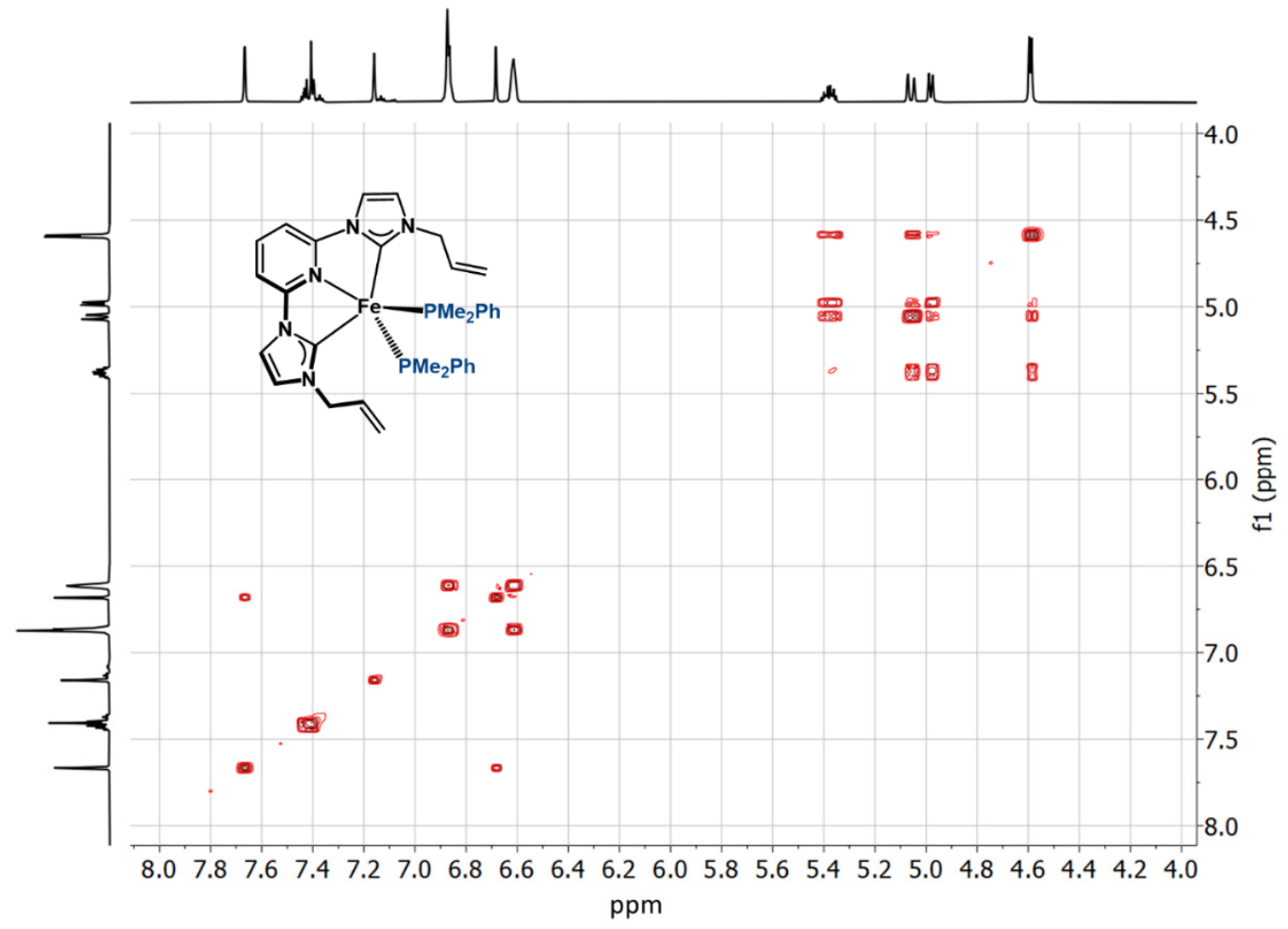

Figure $\mathbf{S 5}{ }^{1} \mathrm{H}-{ }^{1} \mathrm{H}$ COSY spectrum $\left(\mathrm{C}_{6} \mathrm{D}_{6}, 25{ }^{\circ} \mathrm{C}, 700 \mathrm{MHz}\right)$ of $\left({ }^{\text {allyl }} \mathrm{CNC}\right) \mathrm{Fe}\left(\mathrm{PMe}_{2} \mathrm{Ph}\right)_{2}\left(3-\mathrm{PMe} \mathbf{P}_{2} \mathrm{Ph}\right)$. 


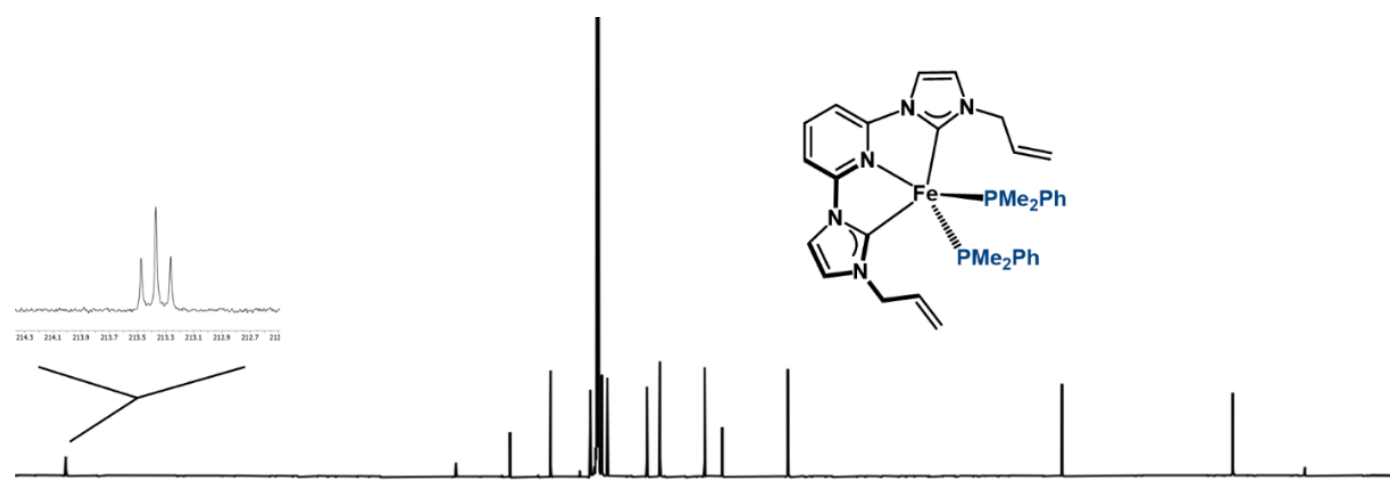

$\begin{array}{llllllllllllllllllllll}20 & 210 & 200 & 190 & 180 & 170 & 160 & 150 & 140 & 130 & 120 & \begin{array}{c}110 \\ \mathrm{ppm}\end{array} & 100 & 90 & 80 & 70 & 60 & 50 & 40 & 30 & 20 & 10\end{array}$

Figure $56{ }^{13} \mathrm{C}$ NMR spectrum $\left(\mathrm{C}_{6} \mathrm{D}_{6}, 25{ }^{\circ} \mathrm{C}, 126 \mathrm{MHz}\right)$ of (allyl $\left.\mathrm{CNC}\right) \mathrm{Fe}\left(\mathrm{PMe} \mathrm{Ph}_{2}\right)_{2}\left(3-\mathrm{PMe} \mathrm{P}_{2} \mathrm{Ph}\right)$.

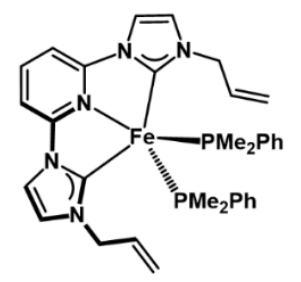

$\begin{array}{lllllllllllllllllllllllll}190 & 180 & 170 & 160 & 150 & 140 & 130 & 120 & 110 & 100 & 90 & 80 & 70 & 60 & 50 & 40 & 30 & 20 & 10 & 0 & -10 & -20 & -30 & -40 \\ \mathrm{ppm}\end{array}$

Figure $\mathbf{S 7}{ }^{31} \mathrm{P}$ NMR spectrum $\left(\mathrm{C}_{6} \mathrm{D}_{6}, 25{ }^{\circ} \mathrm{C}, 202 \mathrm{MHz}\right)$ of $\left({ }^{\text {allyl }} \mathrm{CNC}\right) \mathrm{Fe}\left(\mathrm{PMe}{ }_{2} \mathrm{Ph}\right)_{2}\left(3-\mathrm{PMe} \mathbf{P}_{2} \mathrm{Ph}\right)$.

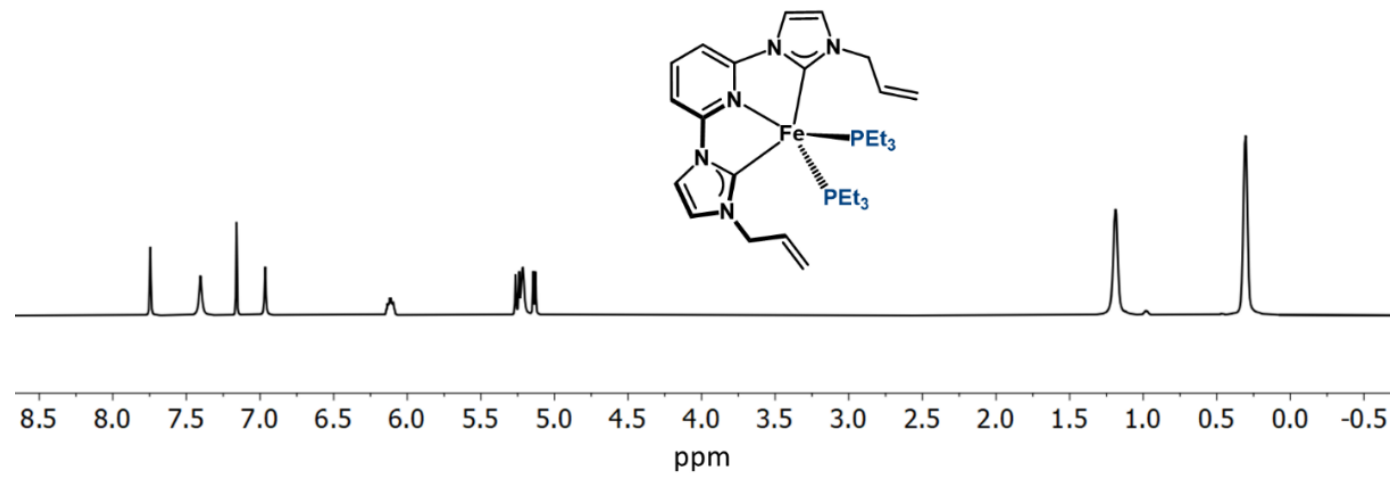

Figure S8 ${ }^{1} \mathrm{H}$ NMR spectrum $\left(\mathrm{C}_{6} \mathrm{D}_{6}, 25{ }^{\circ} \mathrm{C}, 700 \mathrm{MHz}\right)$ of $\left({ }^{\text {allyl }} \mathrm{CNC}\right) \mathrm{Fe}\left(\mathrm{PEt}_{3}\right)_{2}\left(3-\mathrm{PEt}_{3}\right)$. 


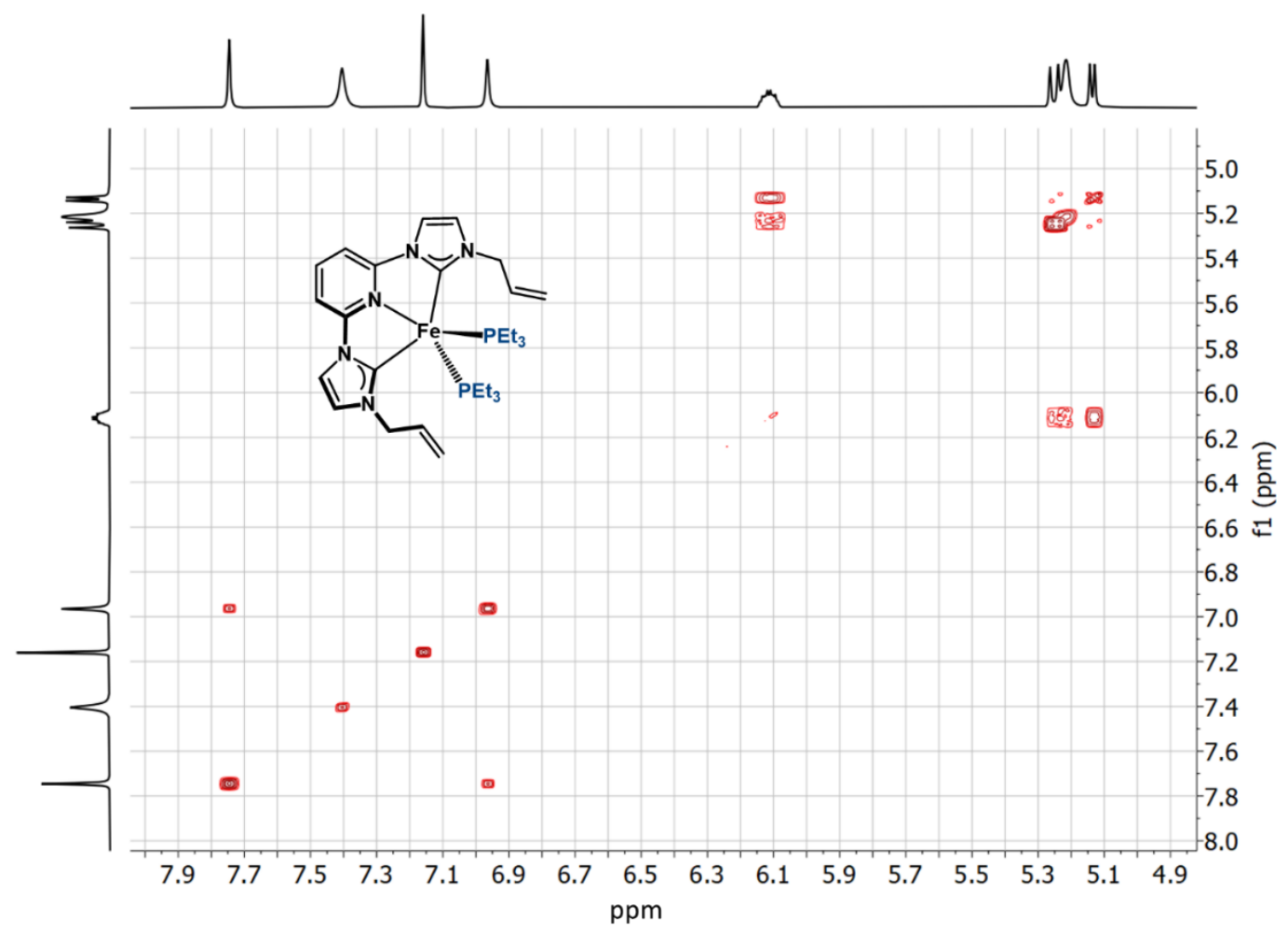

Figure $59{ }^{1} \mathrm{H}-{ }^{1} \mathrm{H}$ COSY spectrum $\left(\mathrm{C}_{6} \mathrm{D}_{6}, 25{ }^{\circ} \mathrm{C}, 700 \mathrm{MHz}\right)$ of $\left({ }^{\text {allyl }} \mathrm{CNC}\right) \mathrm{Fe}\left(\mathrm{PEt}_{3}\right)_{2}\left(3-\mathrm{PEt}_{3}\right)$.
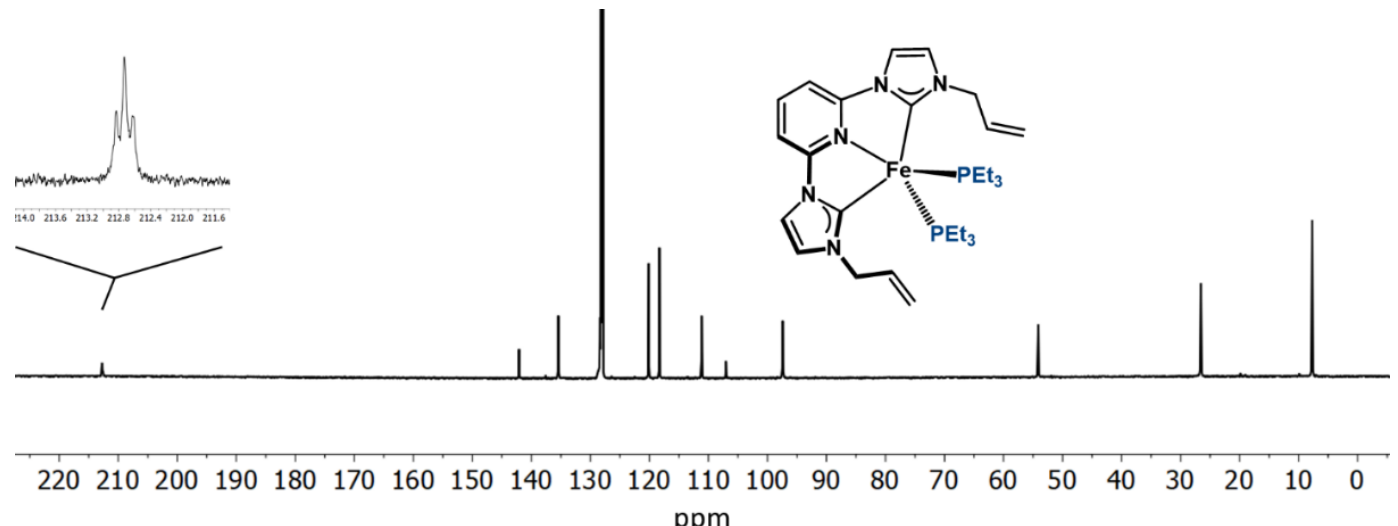

Figure $\mathbf{S} 10{ }^{13} \mathrm{C}$ NMR spectrum $\left(\mathrm{C}_{6} \mathrm{D}_{6}, 25{ }^{\circ} \mathrm{C}, 126 \mathrm{MHz}\right)$ of $\left({ }^{\text {allyly }} \mathrm{CNC}\right) \mathrm{Fe}\left(\mathrm{PEt}_{3}\right)_{2}\left(3-\mathrm{PEt}_{3}\right)$. 


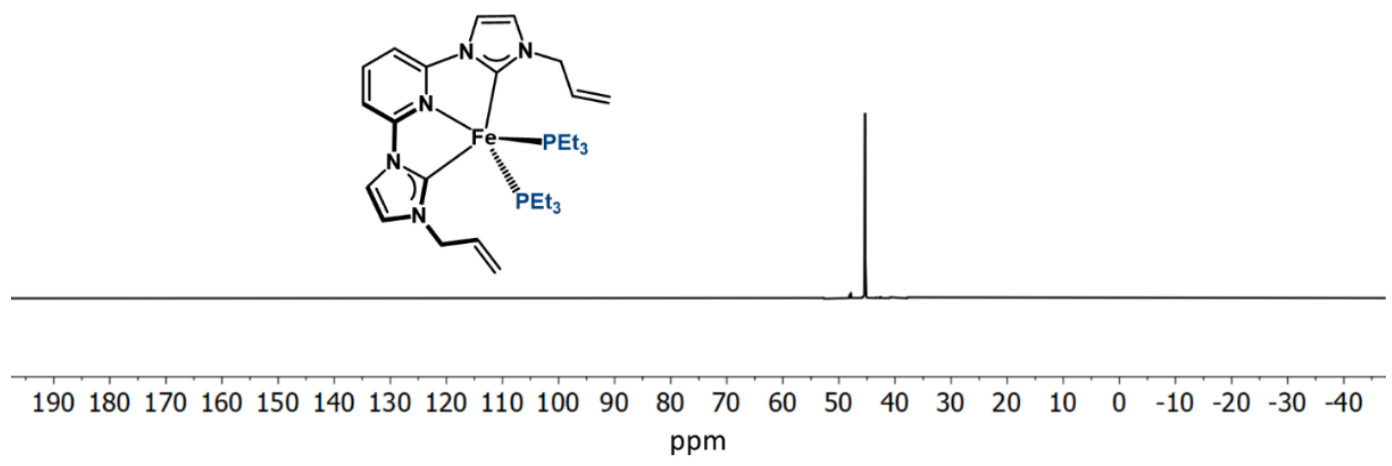

Figure $\mathrm{S} 11{ }^{31} \mathrm{P}$ NMR spectrum $\left(\mathrm{C}_{6} \mathrm{D}_{6}, 25{ }^{\circ} \mathrm{C}, 202 \mathrm{MHz}\right)$ of $\left({ }^{\mathrm{ally}} \mathrm{CNC}\right) \mathrm{Fe}\left(\mathrm{PEt}_{3}\right)_{2}\left(3-\mathrm{PEt}_{3}\right)$.

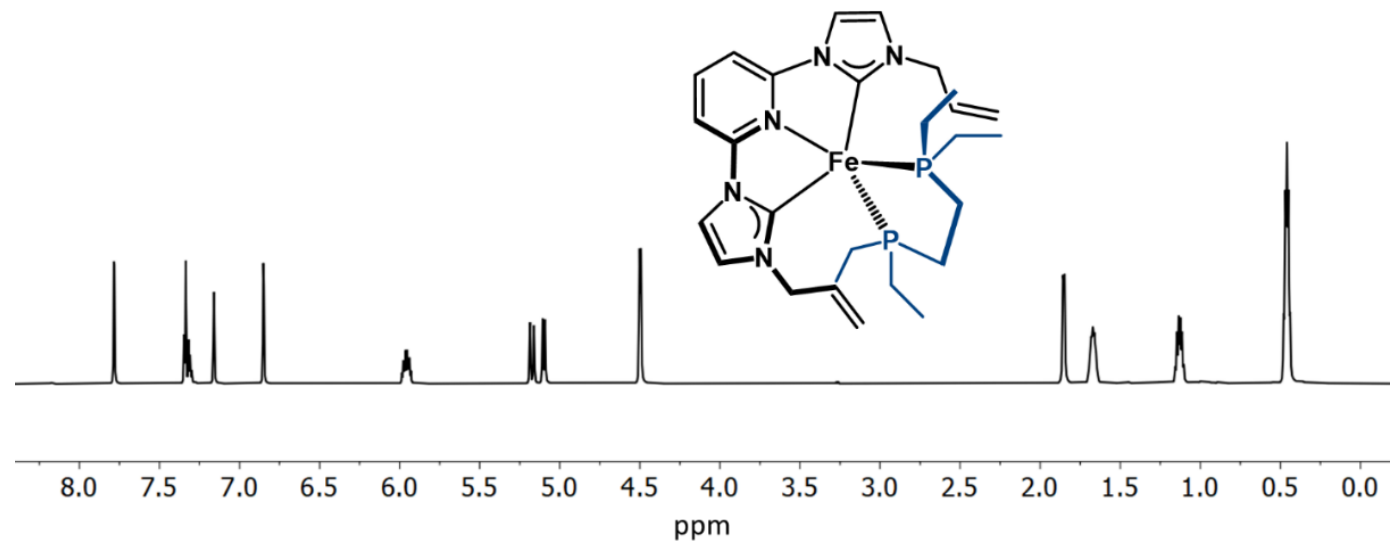

Figure S12 ${ }^{1} \mathrm{H}$ NMR spectrum $\left(\mathrm{C}_{6} \mathrm{D}_{6}, 25^{\circ} \mathrm{C}, 700 \mathrm{MHz}\right)$ of (allyl $\left.\mathrm{CNC}\right) \mathrm{Fe}$ (depe) (3-depe).

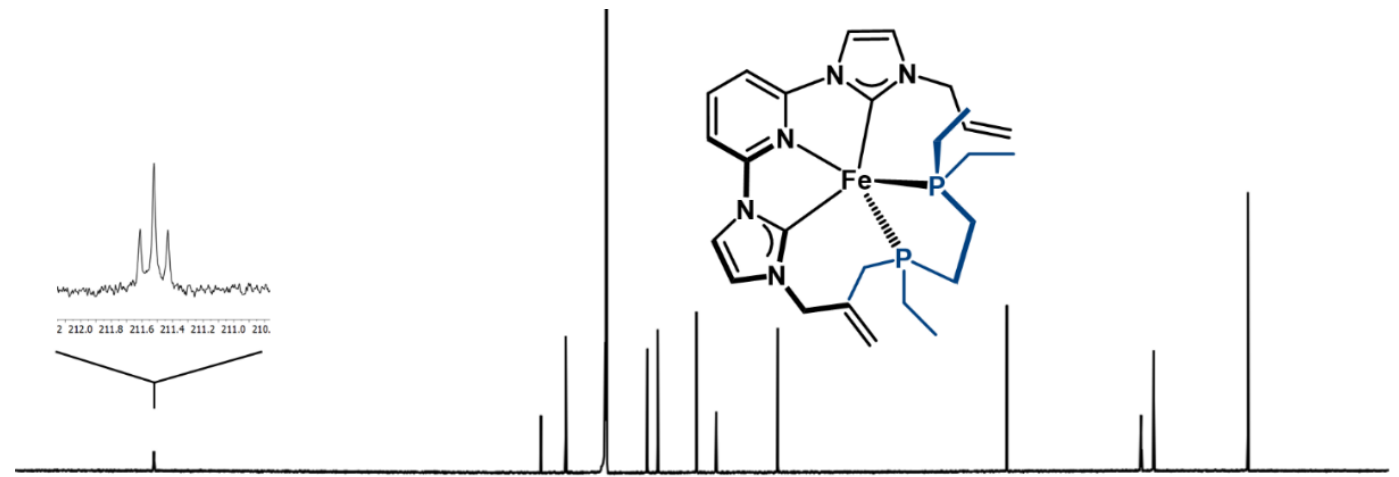

$230220210200190180170160150140130120110100 \quad 90 \quad 80 \quad 70 \quad 60 \quad 50 \quad 40 \quad 30 \begin{array}{lllll}10 & 0 & -10\end{array}$ ppm

Figure $\mathrm{S} 13{ }^{13} \mathrm{C}$ NMR spectrum $\left(\mathrm{C}_{6} \mathrm{D}_{6}, 25^{\circ} \mathrm{C}, 126 \mathrm{MHz}\right)$ of (allyl $\left.\mathrm{CNC}\right) \mathrm{Fe}($ depe) (3-depe). 


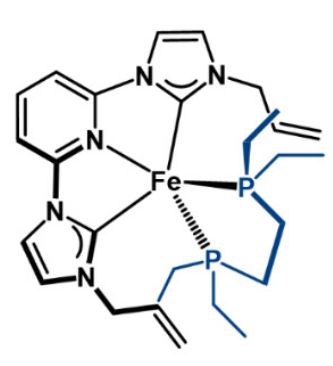

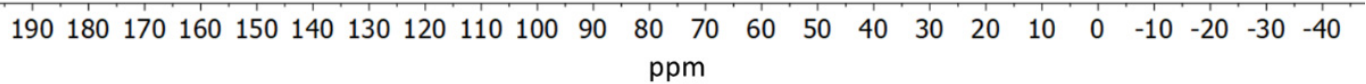

Figure S14 ${ }^{31} \mathrm{P}$ NMR spectrum $\left(\mathrm{C}_{6} \mathrm{D}_{6}, 25^{\circ} \mathrm{C}, 202 \mathrm{MHz}\right)$ of (allyl $\left.\mathrm{CNC}\right) \mathrm{Fe}($ depe) (3-depe).

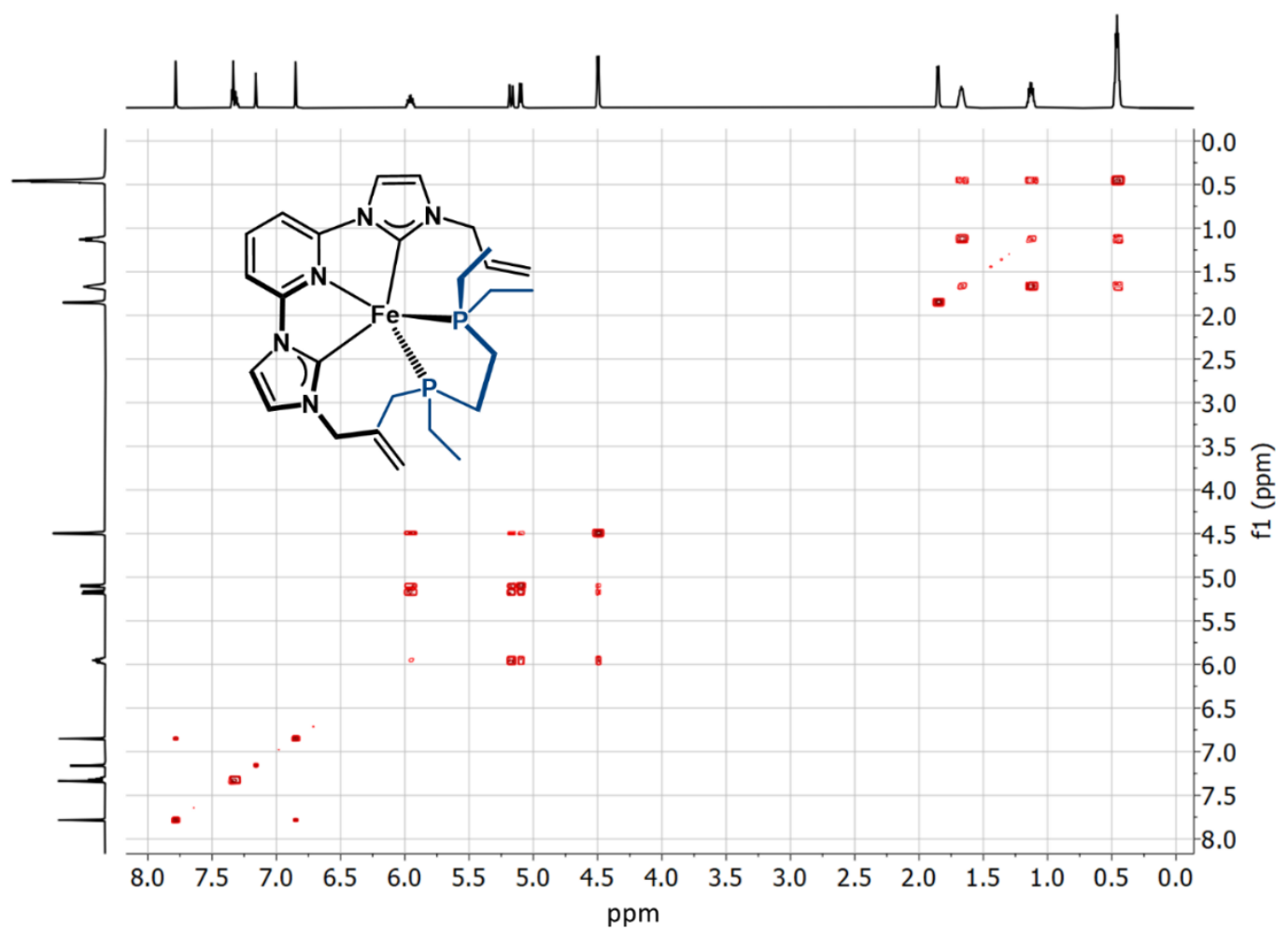

Figure $\mathrm{S} 15{ }^{1} \mathrm{H}-{ }^{1} \mathrm{H}$ COSY spectrum $\left(\mathrm{C}_{6} \mathrm{D}_{6}, 25{ }^{\circ} \mathrm{C}, 700 \mathrm{MHz}\right.$ ) of (allyl $\left.\mathrm{CNC}\right) \mathrm{Fe}($ depe) (3-depe). 

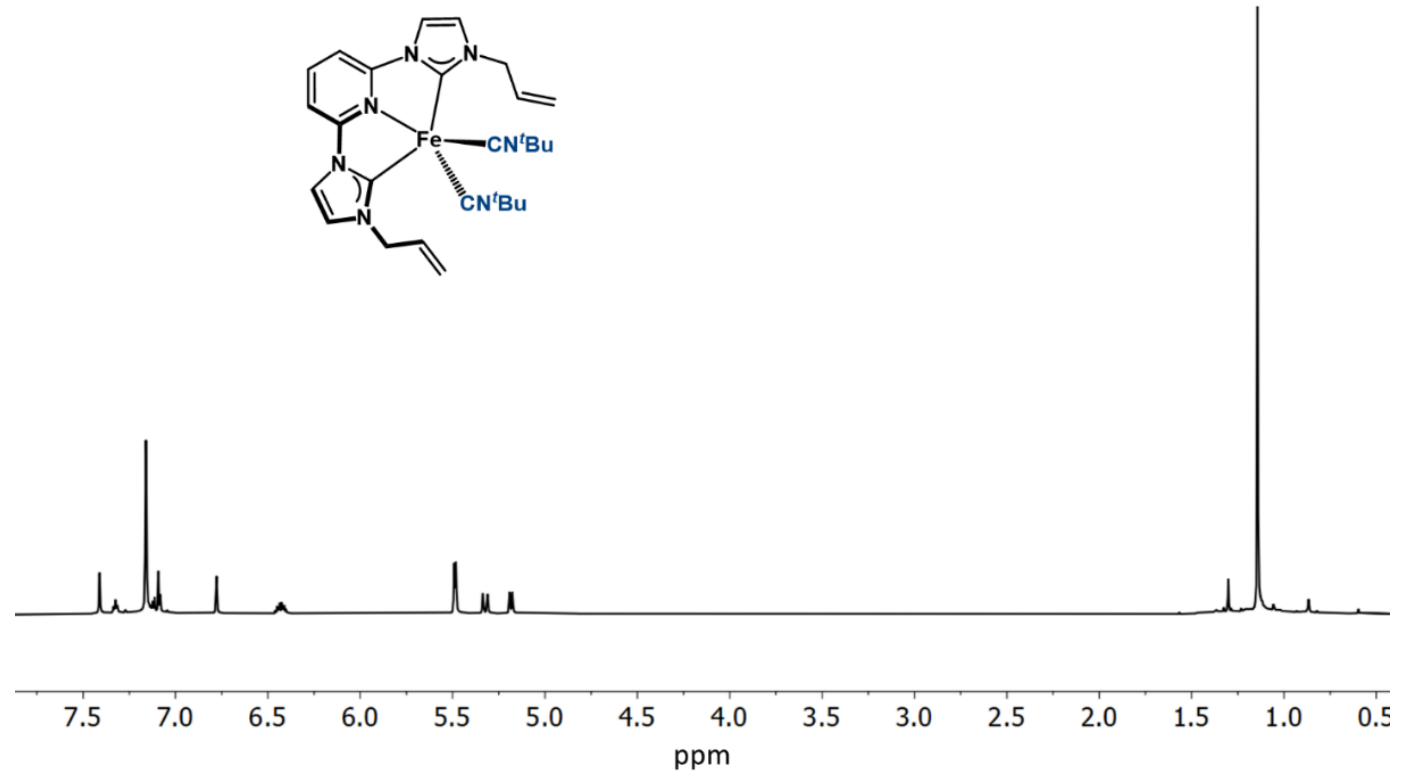

Figure $\mathrm{S} 16{ }^{1} \mathrm{H}$ NMR spectrum $\left(\mathrm{C}_{6} \mathrm{D}_{6}, 25^{\circ} \mathrm{C}, 500 \mathrm{MHz}\right)$ of $\left({ }^{\text {allyl }} \mathrm{CNC}\right) \mathrm{Fe}\left(\mathrm{CN}^{t} \mathrm{Bu}\right)_{2}\left(4^{-}{ }^{t} \mathrm{Bu}\right)$.

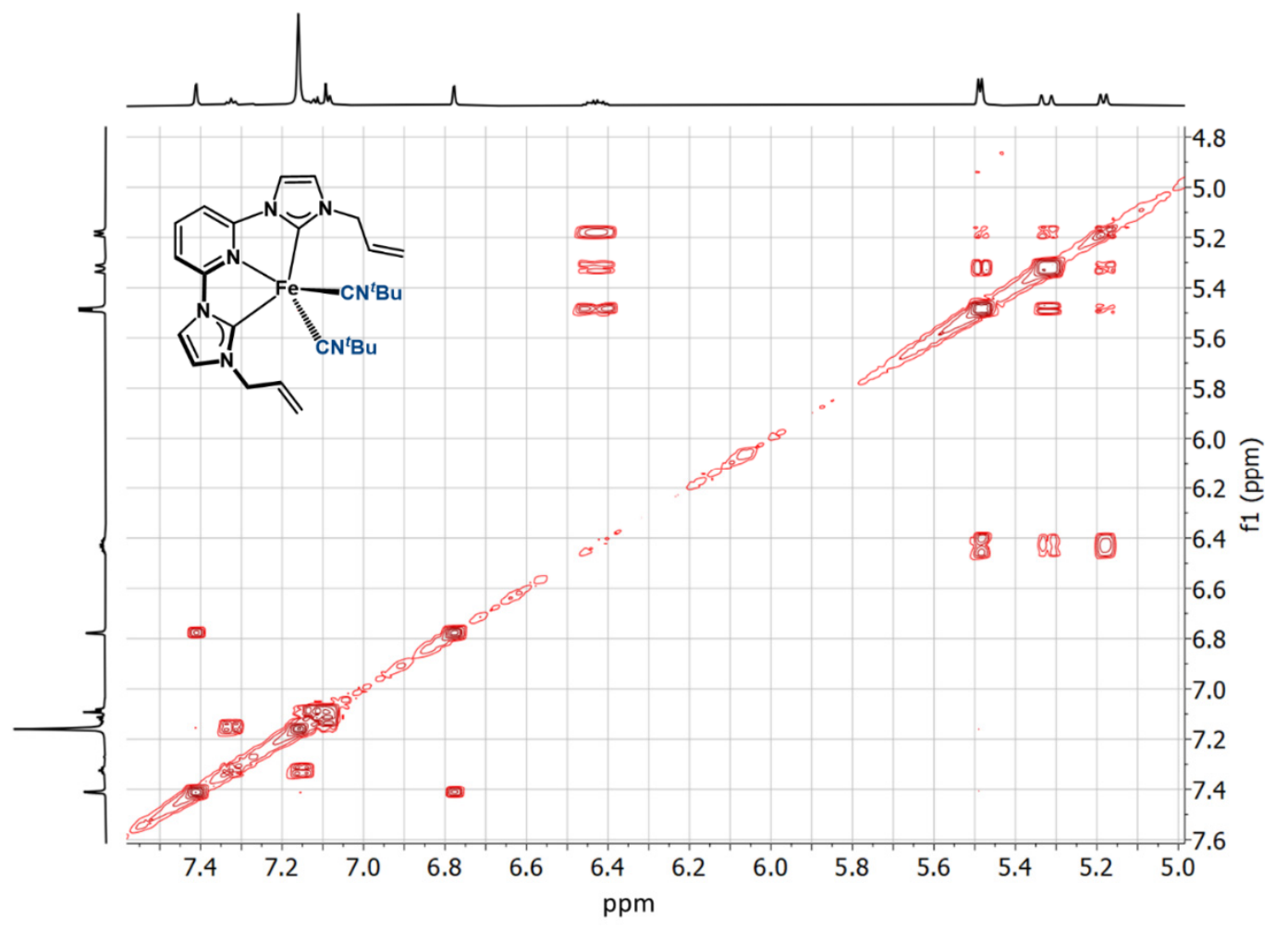

Figure $\mathbf{S 1 7}{ }^{1} \mathrm{H}-{ }^{1} \mathrm{H}$ COSY spectrum $\left(\mathrm{C}_{6} \mathrm{D}_{6}, 25^{\circ} \mathrm{C}, 700 \mathrm{MHz}\right)$ of $\left({ }^{\text {allyl }} \mathrm{CNC}\right) \mathrm{Fe}\left(\mathrm{CN}^{t} \mathrm{Bu}\right)_{2}\left(4^{\mathrm{t}}{ }^{\mathrm{B} u}\right)$. 


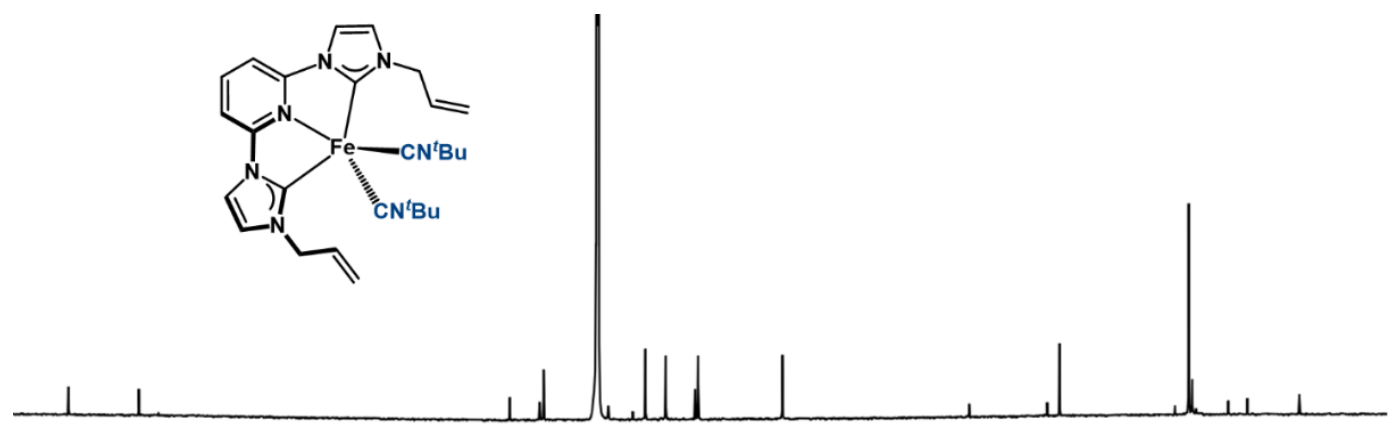

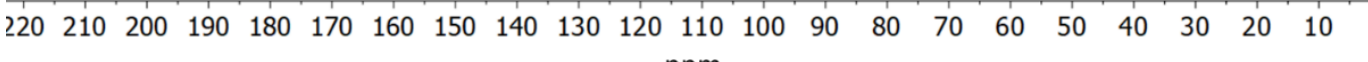
ppm

Figure $\mathbf{S 1 8}{ }^{13} \mathrm{C}$ NMR spectrum $\left(\mathrm{C}_{6} \mathrm{D}_{6}, 25^{\circ} \mathrm{C}, 126 \mathrm{MHz}\right)$ of $\left({ }^{\text {allyl }} \mathrm{CNC}\right) \mathrm{Fe}\left(\mathrm{CN}^{\mathrm{t}} \mathrm{Bu}\right)_{2}\left(4-{ }^{\mathrm{t}} \mathrm{Bu}\right)$.
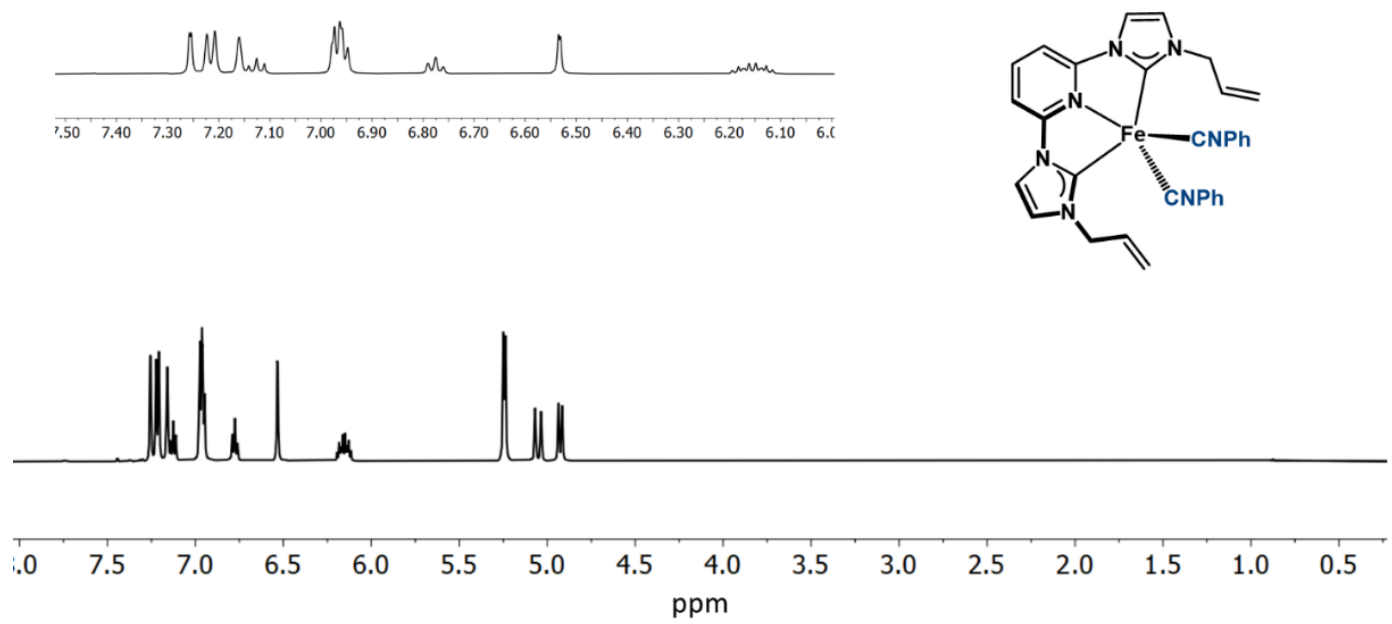

Figure $S 19{ }^{1} \mathrm{H}$ NMR spectrum $\left(\mathrm{C}_{6} \mathrm{D}_{6}, 25^{\circ} \mathrm{C}, 500 \mathrm{MHz}\right)$ of (allyl $\left.\mathrm{CNC}\right) \mathrm{Fe}(\mathrm{CNPh})_{2}(4-\mathrm{Ph})$.
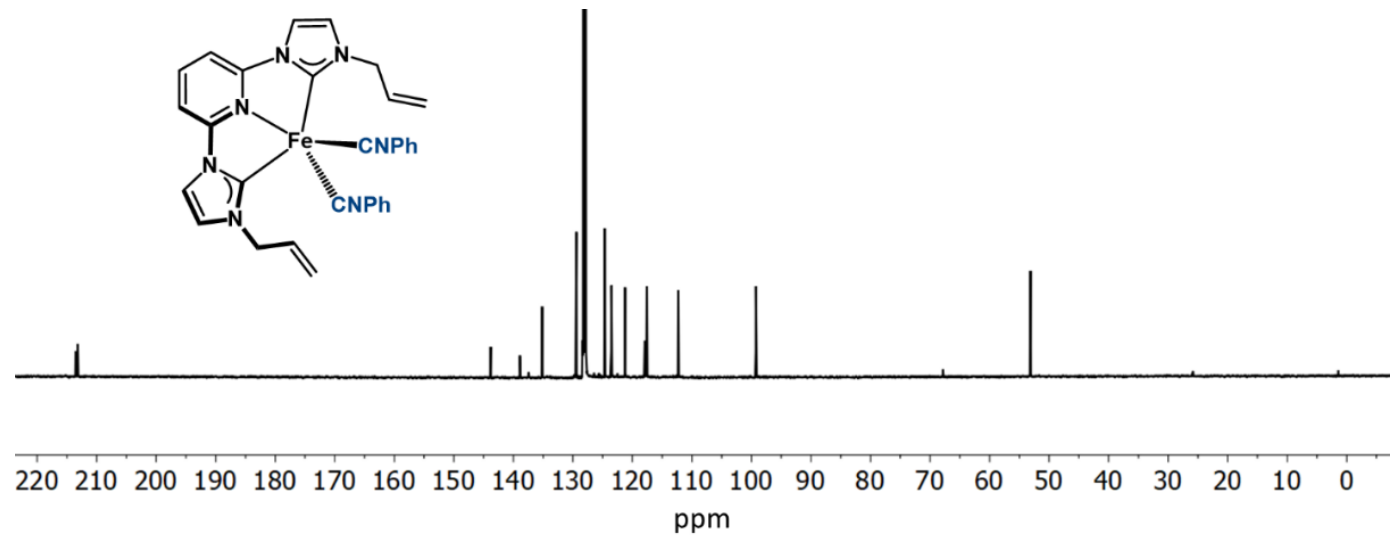

Figure $\mathbf{S 2 0}{ }^{13} \mathrm{C}$ NMR spectrum $\left(\mathrm{C}_{6} \mathrm{D}_{6}, 25{ }^{\circ} \mathrm{C}, 126 \mathrm{MHz}\right)$ of $\left({ }^{\text {allyl }} \mathrm{CNC}\right) \mathrm{Fe}(\mathrm{CNPh})_{2}(4-\mathrm{Ph})$. 


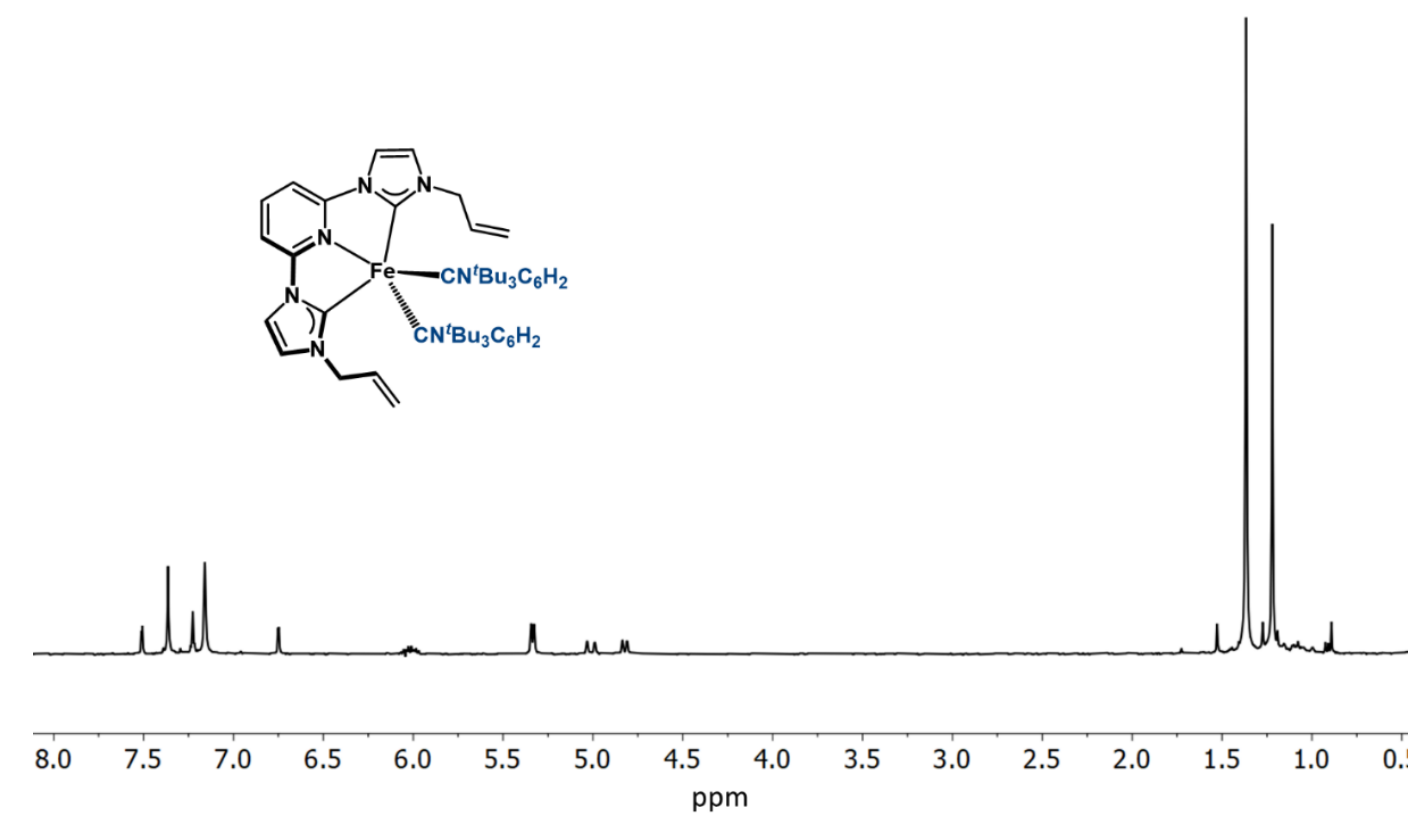

Figure S21 ${ }^{1} \mathrm{H}$ NMR spectrum $\left(\mathrm{C}_{6} \mathrm{D}_{6}, 25^{\circ} \mathrm{C}, 500 \mathrm{MHz}\right)$ of $\left({ }^{\text {allylyl }} \mathrm{CNC}\right) \mathrm{Fe}\left(\mathrm{CN}^{t} \mathrm{Bu}_{3} \mathrm{C}_{6} \mathrm{H}_{2}\right)_{2}\left(\mathbf{4}^{-}{ }^{t} \mathrm{Bu}_{3} \mathrm{C}_{6} \mathrm{H}_{2}\right)$.

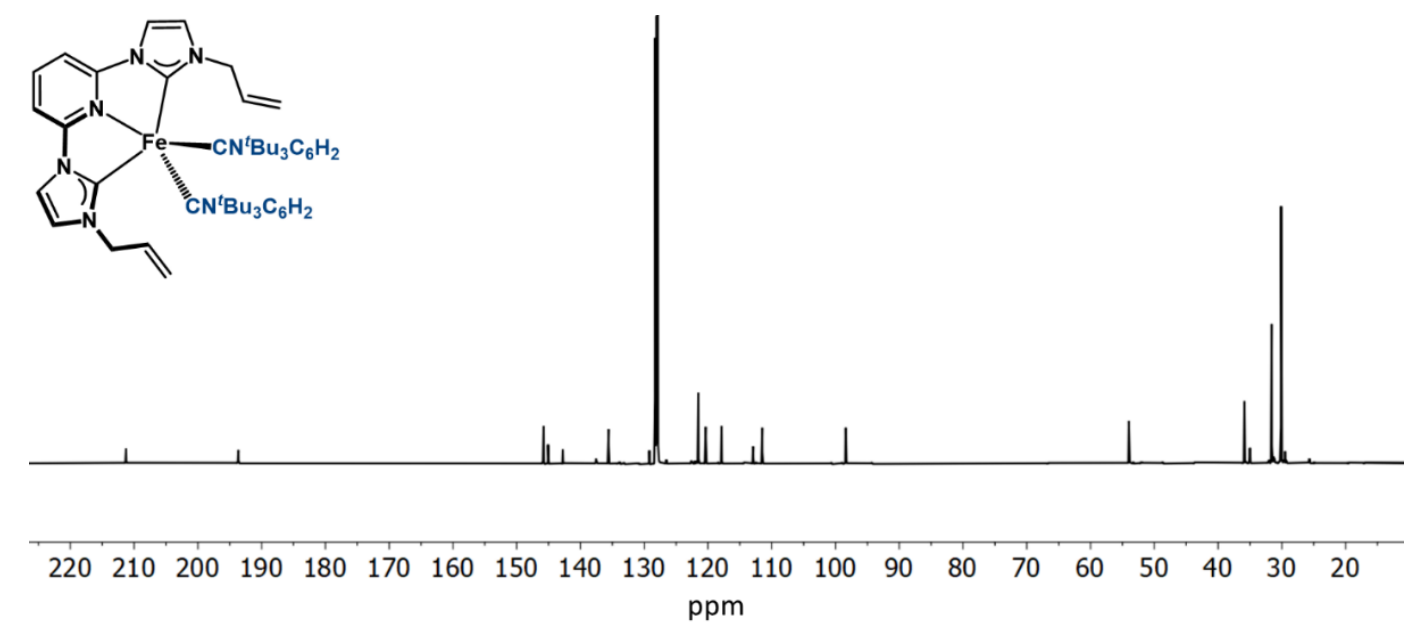

Figure S22 ${ }^{13} \mathrm{C}$ NMR spectrum $\left(\mathrm{C}_{6} \mathrm{D}_{6}, 25{ }^{\circ} \mathrm{C}, 126 \mathrm{MHz}\right)$ of $\left({ }^{\text {allyl }} \mathrm{CNC}\right) \mathrm{Fe}\left(\mathrm{CN}^{t} \mathrm{Bu}_{3} \mathrm{C}_{6} \mathrm{H}_{2}\right)_{2}\left(\mathbf{4}^{-}{ }^{t} \mathrm{Bu}_{3} \mathrm{C}_{6} \mathbf{H}_{2}\right)$. 


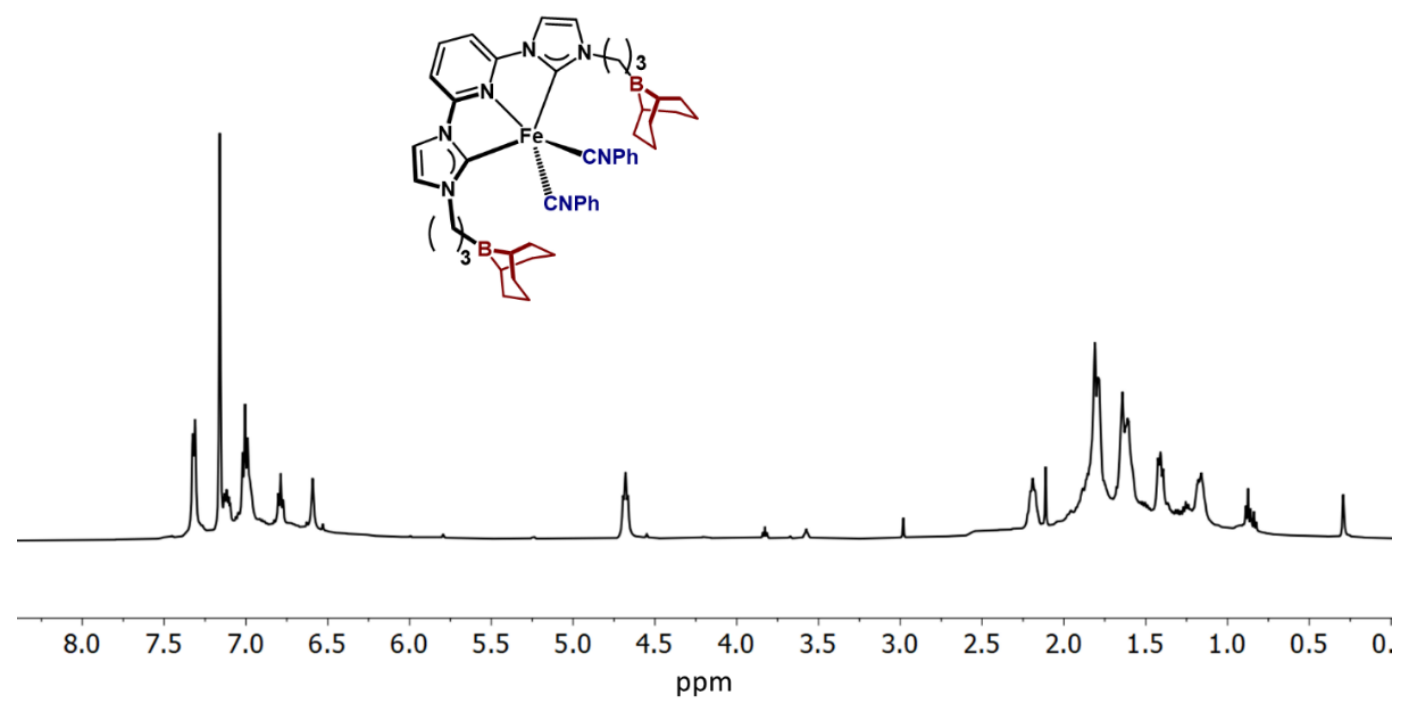

Figure S23 ${ }^{1} \mathrm{H}$ NMR spectrum $\left(\mathrm{C}_{6} \mathrm{D}_{6}, 25^{\circ} \mathrm{C}, 500 \mathrm{MHz}\right)$ of $\left({ }^{\mathrm{BBN}} \mathrm{CNC}\right) \mathrm{Fe}(\mathrm{CNPh})_{2}(5-\mathrm{Ph})$.

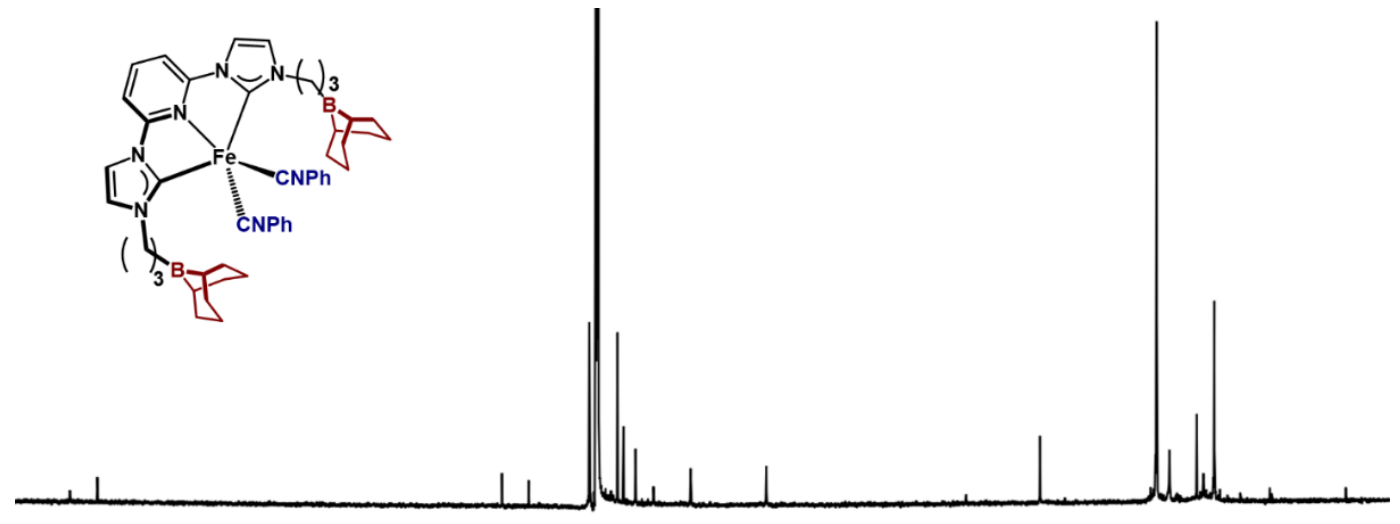

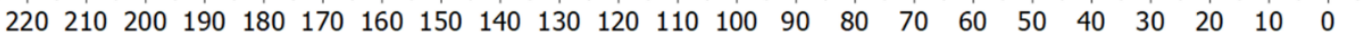
ppm

Figure S24 ${ }^{13} \mathrm{C}$ NMR spectrum $\left(\mathrm{C}_{6} \mathrm{D}_{6}, 25{ }^{\circ} \mathrm{C}, 126 \mathrm{MHz}\right)$ of $\left({ }^{\mathrm{BBN}} \mathrm{CNC}\right) \mathrm{Fe}(\mathrm{CNPh})_{2}(\mathbf{5}-\mathrm{Ph})$. 


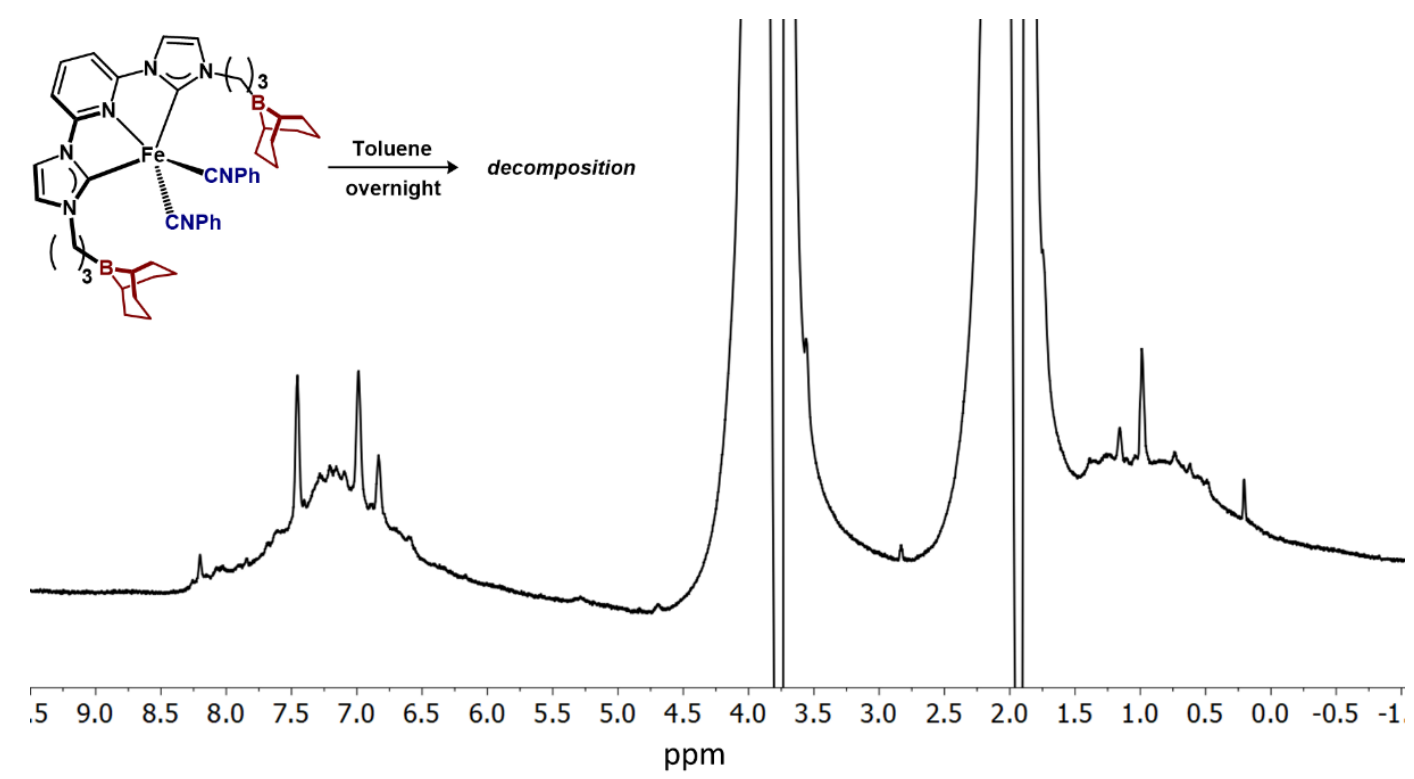

Figure S25 ${ }^{1} \mathrm{H}$ NMR spectrum $\left(\mathrm{THF}, 25{ }^{\circ} \mathrm{C}, 400 \mathrm{MHz}\right)$ of decomposition of $\left({ }^{\mathrm{BBN}} \mathrm{CNC}\right) \mathrm{Fe}(\mathrm{CNPh})_{2}(5-\mathrm{Ph})$.

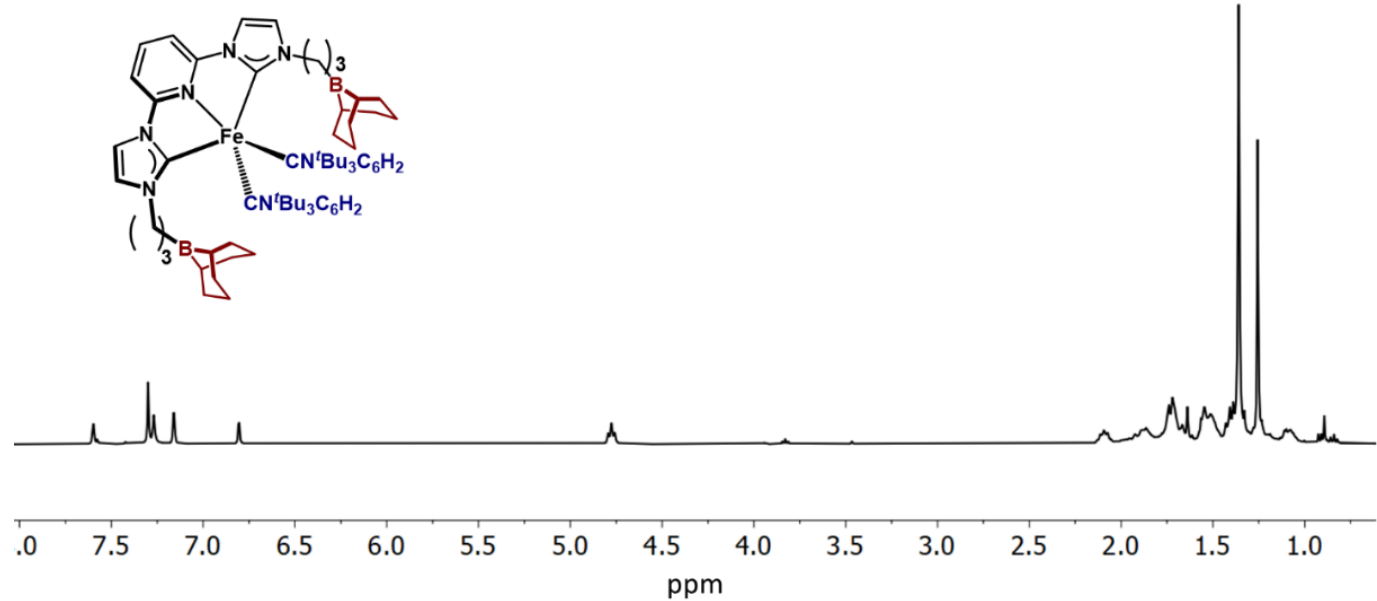

Figure S26 ${ }^{1} \mathrm{H}$ NMR spectrum $\left(\mathrm{C}_{6} \mathrm{D}_{6}, 25{ }^{\circ} \mathrm{C}, 400 \mathrm{MHz}\right)$ of $\left({ }^{\mathrm{BBN}} \mathrm{CNC}\right) \mathrm{Fe}\left(\mathrm{CN}^{t} \mathrm{Bu}_{3} \mathrm{C}_{6} \mathrm{H}_{2}\right)_{2}\left({ }^{-}{ }^{\mathrm{t}} \mathrm{Bu}_{3} \mathrm{C}_{6} \mathrm{H}_{2}\right)$. 


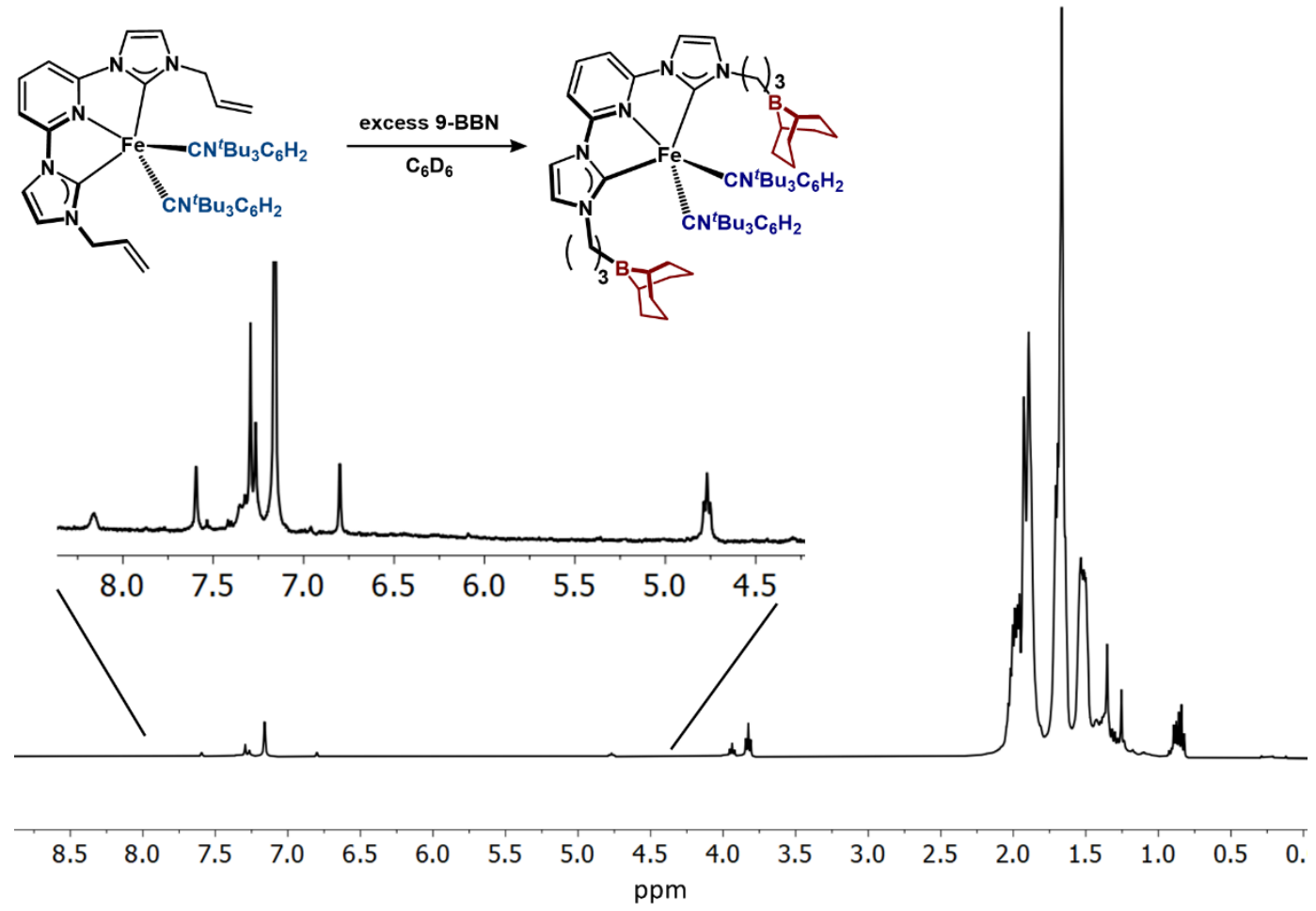

Figure S27 ${ }^{1} \mathrm{H}$ NMR spectrum $\left(\mathrm{C}_{6} \mathrm{D}_{6}, 25{ }^{\circ} \mathrm{C}, 400 \mathrm{MHz}\right)$ of crude reaction of (allyl $\left.\mathrm{CNC}\right) \mathrm{Fe}\left(\mathrm{CN}^{t} \mathrm{Bu}_{3} \mathrm{C}_{6} \mathrm{H}_{2}\right)_{2}$ (4${ }^{t} \mathrm{Bu}_{3} \mathrm{C}_{6} \mathrm{H}_{2}$ ) with excess ( 20 equiv.) 9-BBN to form ( $\left.{ }^{\mathrm{BBN}} \mathrm{CNC}\right) \mathrm{Fe}\left(\mathrm{CN}^{t} \mathrm{Bu}_{3} \mathrm{C}_{6} \mathrm{H}_{2}\right)_{2}\left({ }^{2}-{ }^{t} \mathrm{Bu}_{3} \mathrm{C}_{6} \mathrm{H}_{2}\right)$.
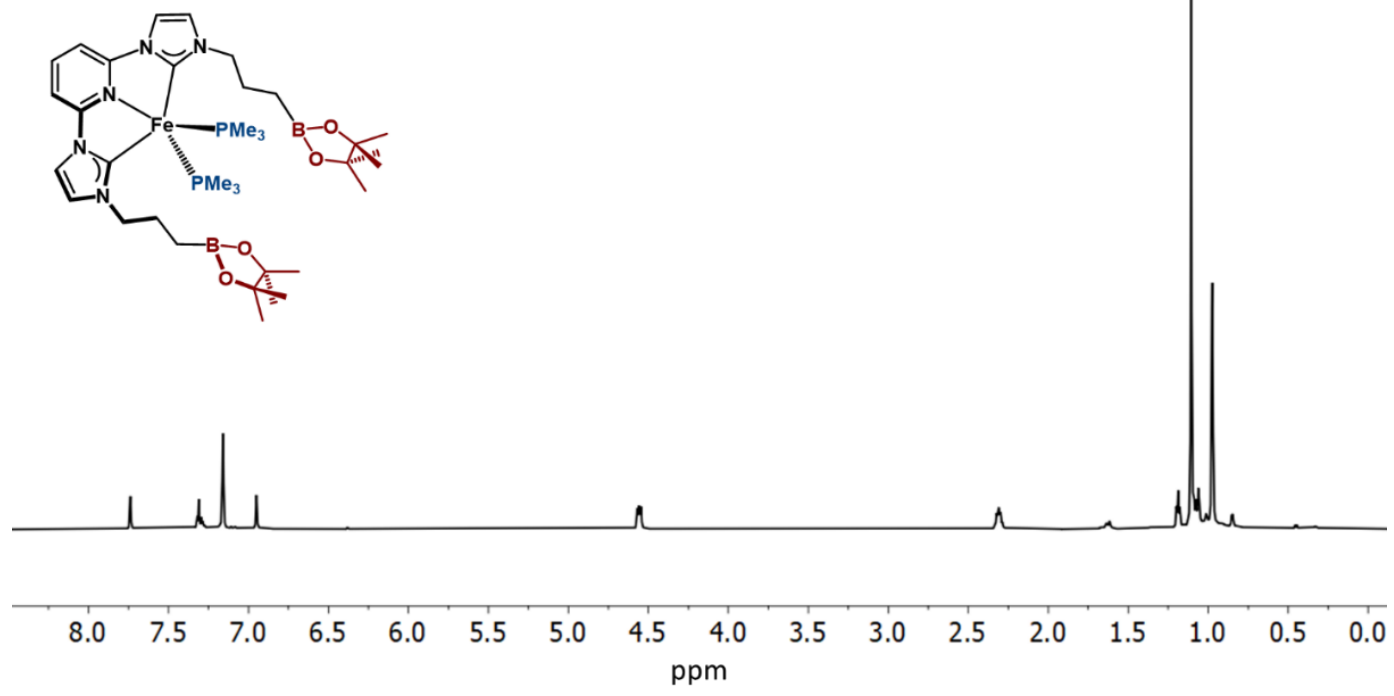

Figure S28 ${ }^{1} \mathrm{H}$ NMR spectrum $\left(\mathrm{C}_{6} \mathrm{D}_{6}, 25{ }^{\circ} \mathrm{C}, 700 \mathrm{MHz}\right)$ of $\left({ }^{\mathrm{BPin}} \mathrm{CNC}\right) \mathrm{Fe}\left(\mathrm{PMe}_{3}\right)_{2}\left(6-\mathrm{PMe}_{3}\right)$. 


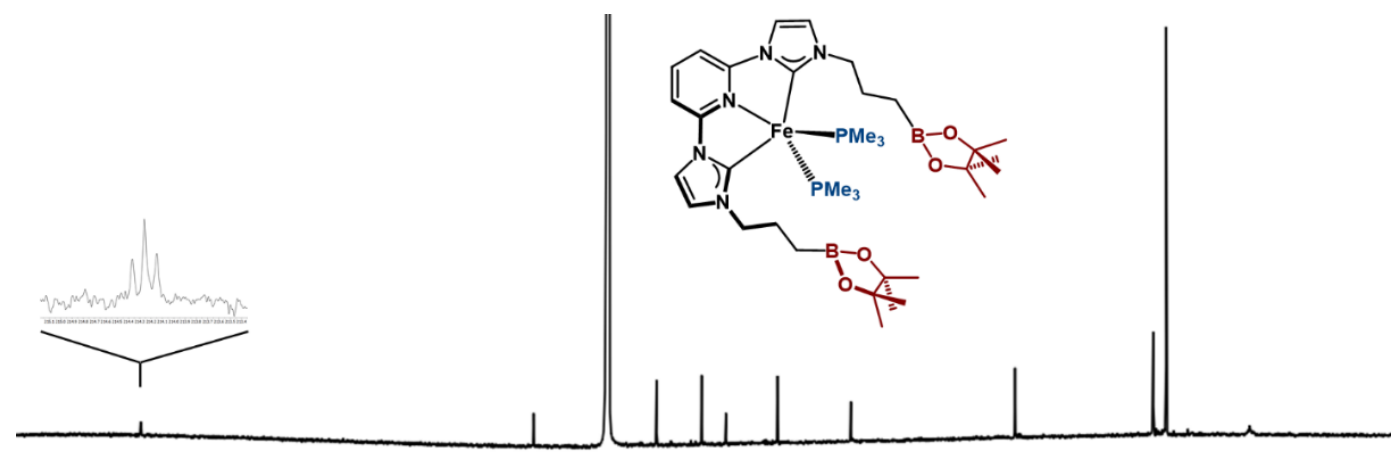

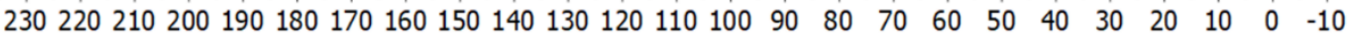
ppm

Figure S29 ${ }^{13} \mathrm{C}$ NMR spectrum $\left(\mathrm{C}_{6} \mathrm{D}_{6}, 25{ }^{\circ} \mathrm{C}, 126 \mathrm{MHz}\right)$ of $\left({ }^{\mathrm{BPin}} \mathrm{CNC}\right) \mathrm{Fe}\left(\mathrm{PMe}_{3}\right)_{2}\left(6-\mathrm{PMe}_{3}\right)$.
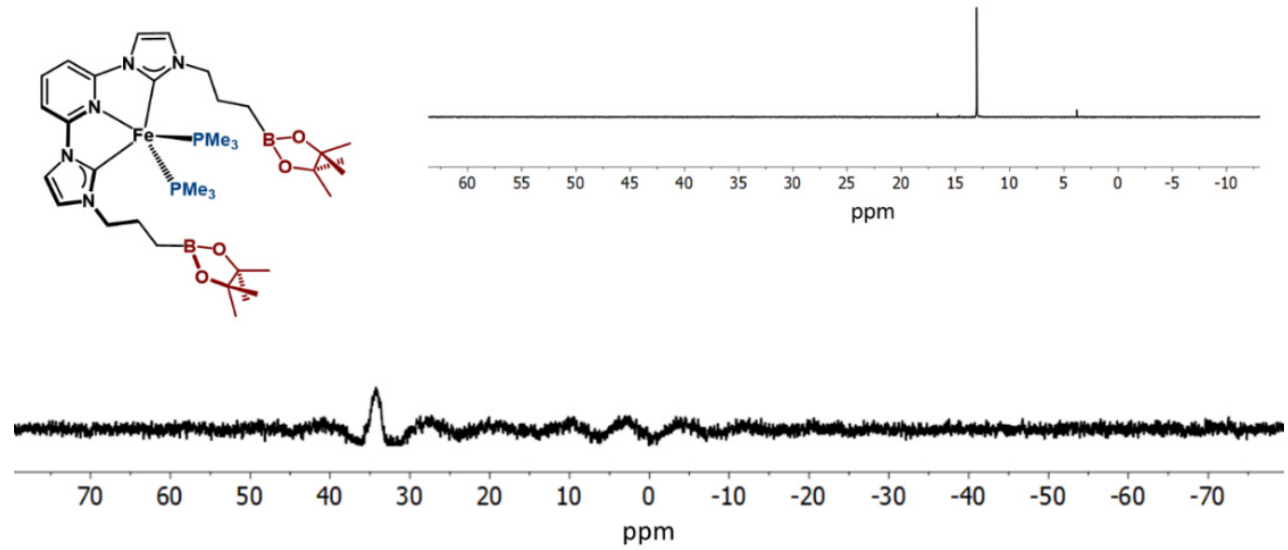

Figure S30 ${ }^{11} \mathrm{~B}$ NMR spectrum (bottom, $\mathrm{C}_{6} \mathrm{D}_{6}, 25{ }^{\circ} \mathrm{C}, 225 \mathrm{MHz}$ ) and ${ }^{31} \mathrm{P}$ NMR spectrum (top, $202 \mathrm{MHz}$ ) of $\left({ }^{\mathrm{BPin}} \mathrm{CNC}\right) \mathrm{Fe}\left(\mathrm{PMe}_{3}\right)_{2}\left(6-\mathrm{PMe}_{3}\right)$.

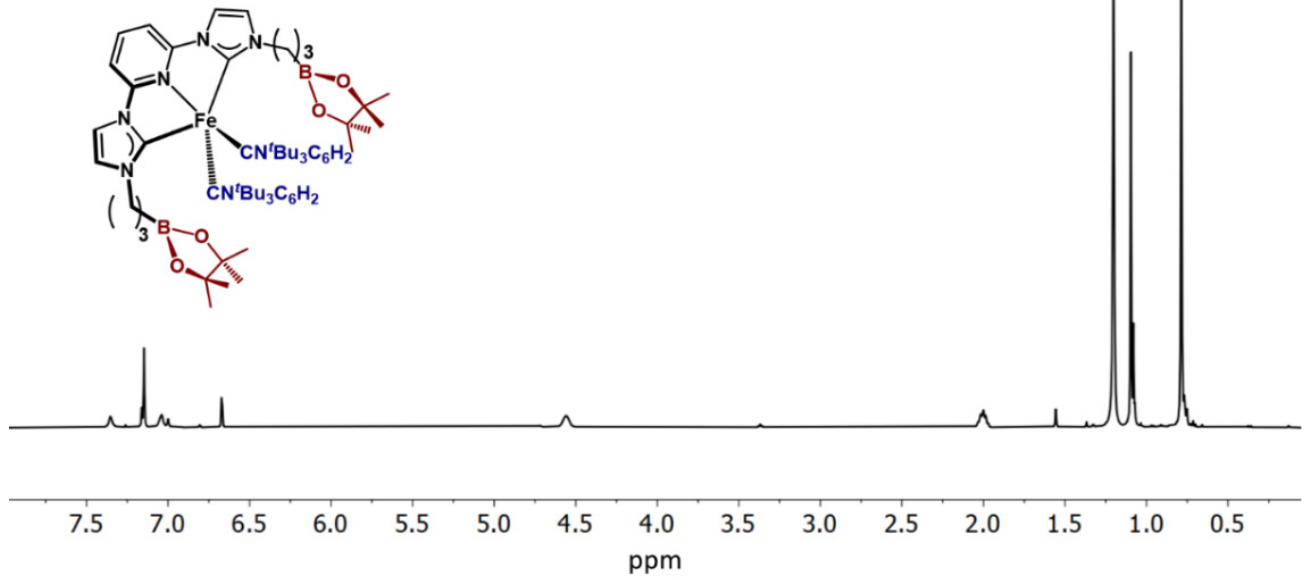

Figure $\mathrm{S} 31{ }^{1} \mathrm{H}$ NMR spectrum $\left(\mathrm{C}_{6} \mathrm{D}_{6}, 25{ }^{\circ} \mathrm{C}, 500 \mathrm{MHz}\right)$ of $\left({ }^{\mathrm{BPin}} \mathrm{CNC}\right) \mathrm{Fe}\left(\mathrm{CN}^{t} \mathrm{Bu}_{3} \mathrm{C}_{6} \mathrm{H}_{2}\right)_{2}\left(6-\mathrm{CN}^{t} \mathrm{Bu}_{3} \mathrm{C}_{6} \mathrm{H}_{2}\right)$. 

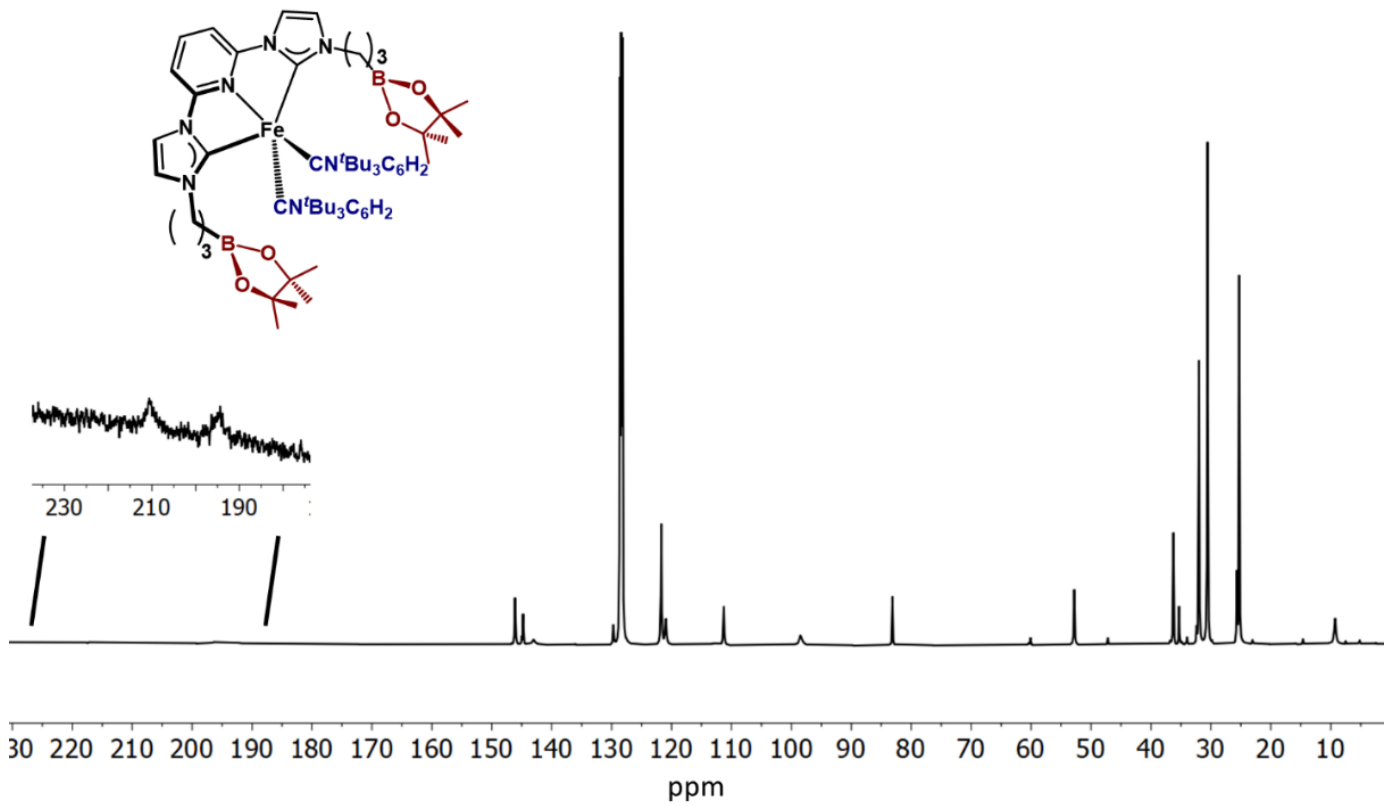

Figure $\mathrm{S} 32{ }^{13} \mathrm{C}$ NMR spectrum $\left(\mathrm{C}_{6} \mathrm{D}_{6}, 25{ }^{\circ} \mathrm{C}, 126 \mathrm{MHz}\right)$ of $\left({ }^{\mathrm{BPin}} \mathrm{CNC}\right) \mathrm{Fe}\left(\mathrm{CN}^{t} \mathrm{Bu}_{3} \mathrm{C}_{6} \mathrm{H}_{2}\right)_{2}\left(6-\mathrm{CN}^{t} \mathrm{Bu}_{3} \mathrm{C}_{6} \mathrm{H}_{2}\right)$.
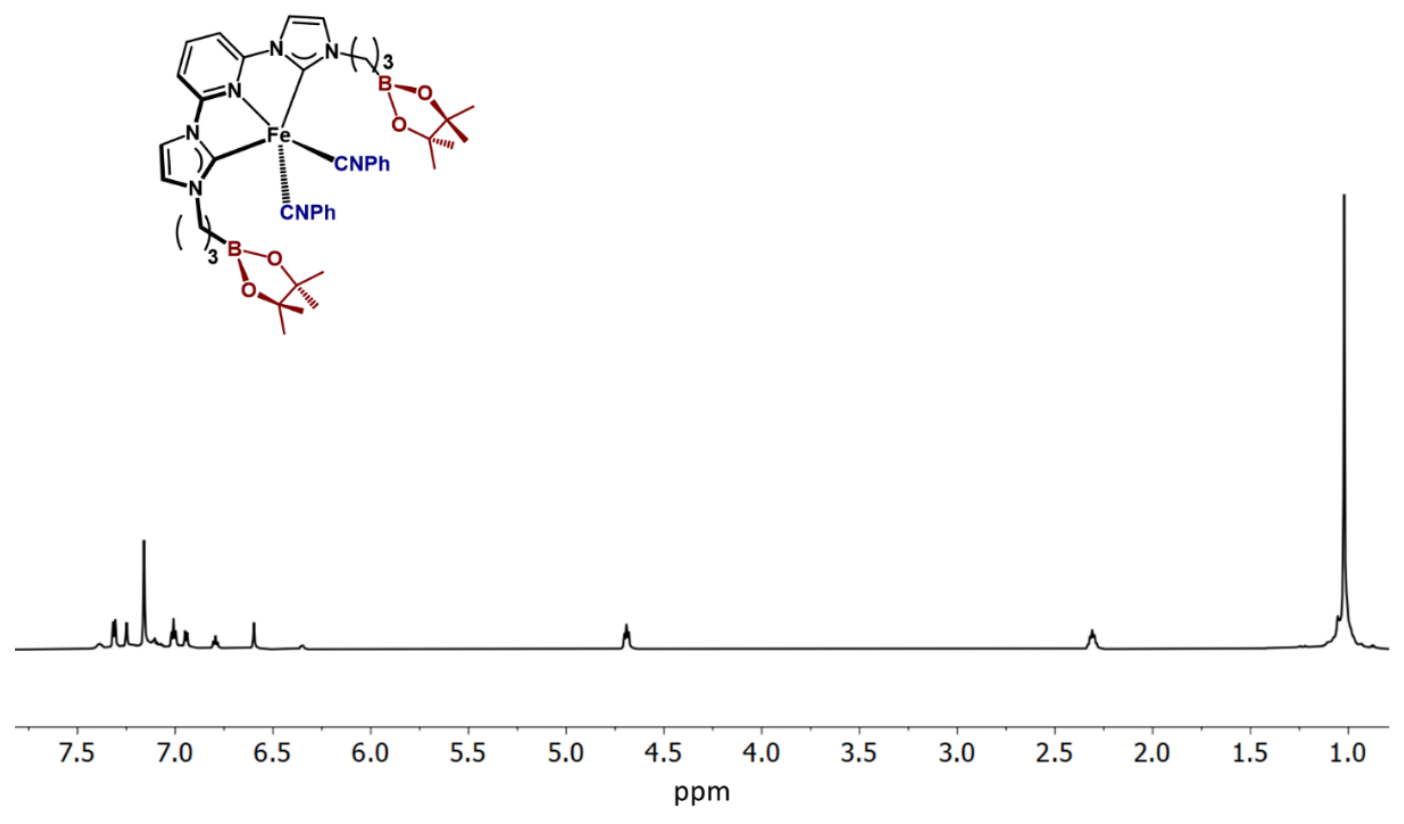

Figure $\mathrm{S} 33^{1} \mathrm{H}$ NMR spectrum $\left(\mathrm{C}_{6} \mathrm{D}_{6}, 25^{\circ} \mathrm{C}, 700 \mathrm{MHz}\right)$ of $\left({ }^{\mathrm{BPin}} \mathrm{CNC}\right) \mathrm{Fe}(\mathrm{CNPh})_{2}(6-\mathrm{CNPh})$. 


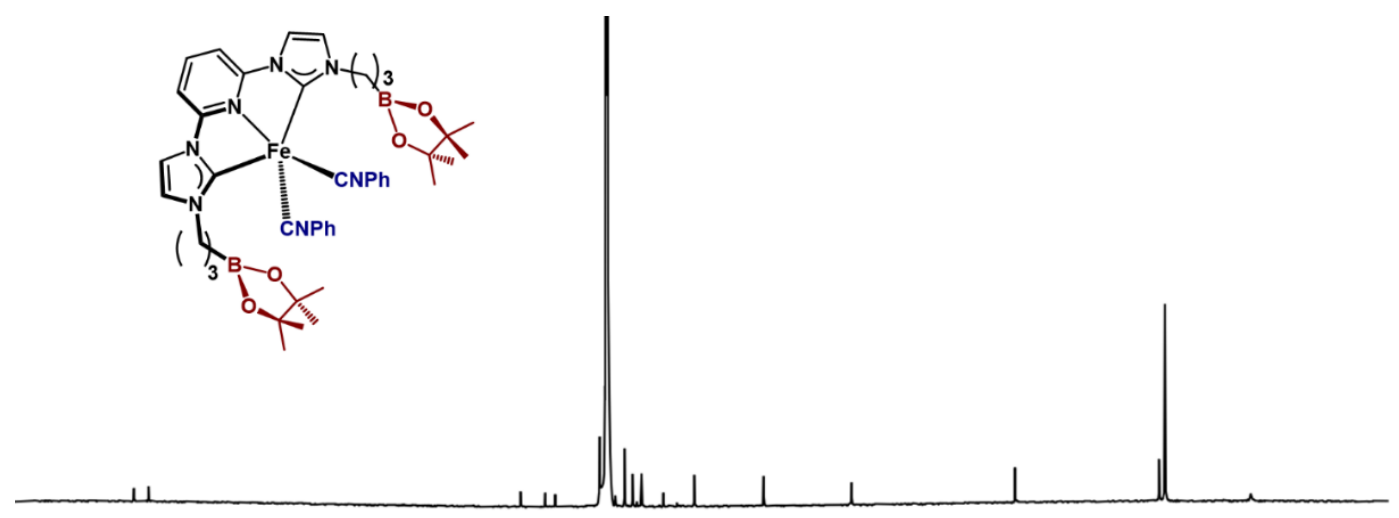

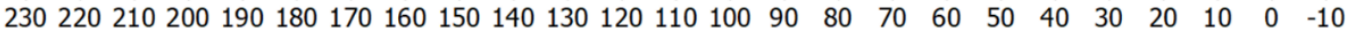
$\mathrm{ppm}$

Figure S34 ${ }^{13} \mathrm{C}$ NMR spectrum $\left(\mathrm{C}_{6} \mathrm{D}_{6}, 25^{\circ} \mathrm{C}, 126 \mathrm{MHz}\right)$ of $\left({ }^{\mathrm{BPin}} \mathrm{CNC}\right) \mathrm{Fe}(\mathrm{CNPh})_{2}(6-\mathrm{CNPh})$.

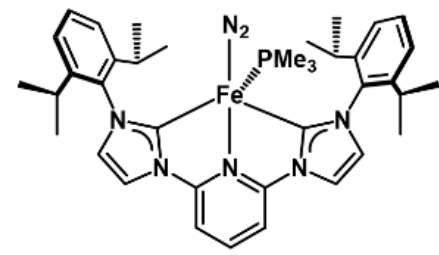

${ }^{1} \mathrm{H}$ NMR $\left(\mathrm{C}_{6} \mathrm{D}_{6}, 25^{\circ} \mathrm{C}\right)$

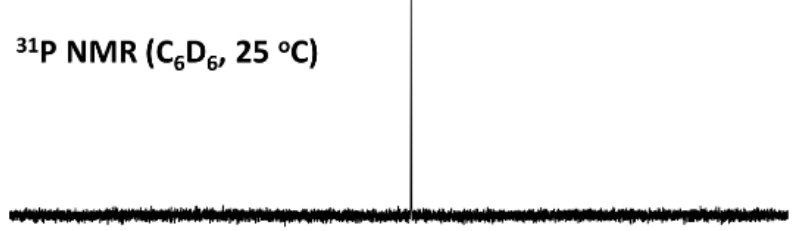

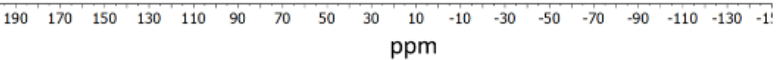

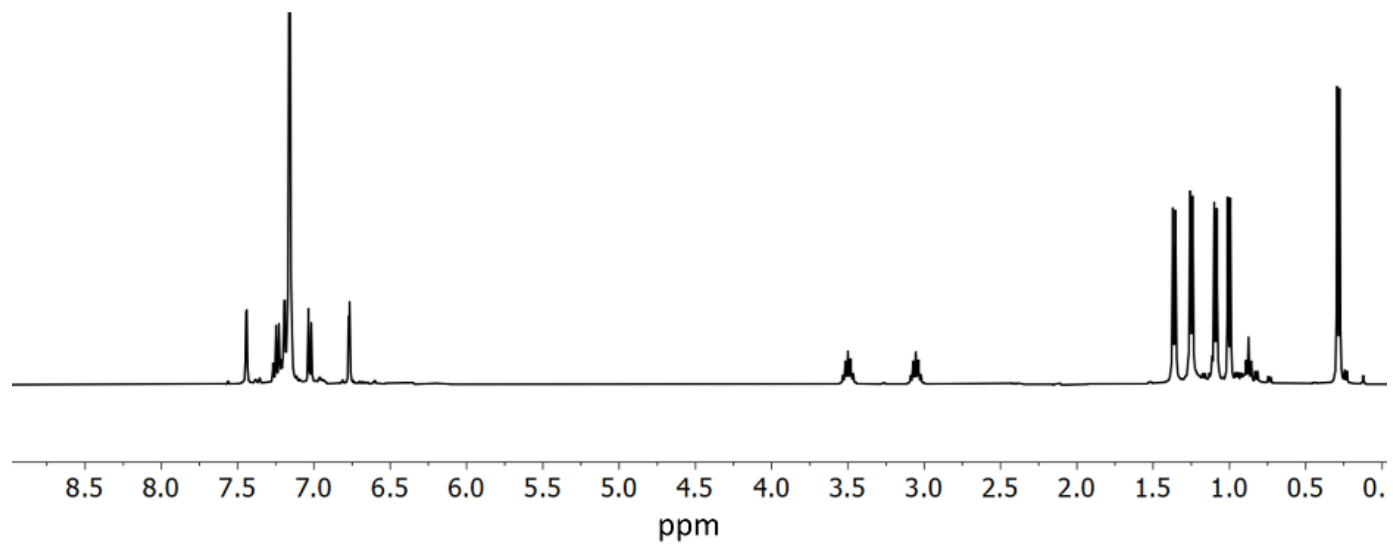

Figure $\mathrm{S} 35{ }^{1} \mathrm{H}$ NMR spectrum (bottom, $\mathrm{C}_{6} \mathrm{D}_{6}, 25{ }^{\circ} \mathrm{C}, 401 \mathrm{MHz}$ ) and ${ }^{31} \mathrm{P} \mathrm{NMR}$ spectrum (top, $162 \mathrm{MHz}$ ) of $\left({ }^{\mathrm{DIPP}} \mathrm{CNC}\right) \mathrm{Fe}\left(\mathrm{PMe}_{3}\right)\left(\mathrm{N}_{2}\right)$. 


\section{Failed Hydroboration Attempts}

Attempted hydroboration of (allyl $\mathrm{CNC}) \mathrm{Fe}\left(\mathrm{PMe}_{3}\right)_{2}$ (3-PMe $)_{3}$. A $20 \mathrm{~mL}$ scintillation vial was charged with

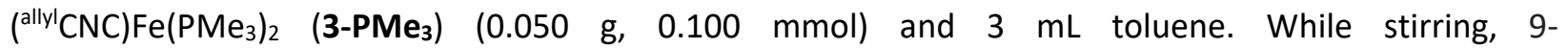
borabicyclo[3.3.1]nonane $(0.025 \mathrm{~g}, 0.205 \mathrm{mmol})$ was added. The reaction was stirred at room temperature. After $60 \mathrm{~min}$, an aliquot was removed and volatiles were removed in vacuo. The material was analyzed by NMR spectroscopy $\left(C_{6} D_{6}\right)$ and revealed the presence of starting material, numerous unidentified species, and the presence of the $\mathrm{Me}_{3} \mathrm{P} \rightarrow 9-\mathrm{BBN}$ adduct. The remaining material was heated to $60{ }^{\circ} \mathrm{C}$ for $90 \mathrm{~min}$, then analyzed by NMR spectroscopy. Analysis revealed that all (allyl $\left.\mathrm{CNC}\right) \mathrm{Fe}\left(\mathrm{PMe}_{3}\right)_{2}(3-$ $\mathrm{PMe}_{3}$ ) was consumed, the major phosphorus containing species was the $\mathrm{Me}_{3} \mathrm{P} \rightarrow 9-\mathrm{BBN}$ adduct, and numerous other unidentifiable species were present. Example spectra are provided below.

Attempted hydroboration of (allyl $\mathrm{CNC}) \mathrm{Fe}\left(\mathrm{PMe}_{2} \mathrm{Ph}\right)_{2}$ (3-PMe $\left.{ }_{2} \mathrm{Ph}\right)$. A $20 \mathrm{~mL}$ scintillation vial was charged with (allyl $\mathrm{CNC}) \mathrm{Fe}\left(\mathrm{PMe}_{2} \mathrm{Ph}\right)_{2}$ (3-PMe $\left.{ }_{2} \mathrm{Ph}\right)(0.019 \mathrm{~g}, 0.030 \mathrm{mmol})$ and $2 \mathrm{~mL}$ benzene. While stirring, 9borabicyclo[3.3.1]nonane $(0.010 \mathrm{~g}, 0.082 \mathrm{mmol})$ was added. The reaction was stirred at room temperature for $16 \mathrm{hr}$. An aliquot was removed and analyzed by NMR spectroscopy. Analysis revealed that all (allyl $\mathrm{CNC}) \mathrm{Fe}\left(\mathrm{PMe}_{2} \mathrm{Ph}\right)_{2}$ (3-PMe ${ }_{2} \mathrm{Ph}$ ) was consumed, the major phosphorus containing species was the $\mathrm{PMe}_{2} \mathrm{Ph} \rightarrow 9-\mathrm{BBN}$ adduct, and numerous other unidentifiable species were present. Example spectra are provided below. The remaining material was left in solution, undisturbed for $48 \mathrm{hr}$, resulting in the deposition of light orange crystals. These samples were analyzed by X-ray diffraction and revealed the material to be $\left[\left({ }^{\mathrm{BBN}} \mathrm{CNC}\right) \mathrm{Fe}\left(\mathrm{PMe}{ }_{2} \mathrm{Ph}\right)_{2}(\mathrm{H})(\mathrm{OH})\right]_{2}$ (see crystallographic information below). The NMR analysis suggests this structure does not represent the bulk of the material.

Attempted hydroboration of (allyl $\mathrm{CNC}) \mathrm{Fe}\left(\mathrm{PEt}_{3}\right)_{2}$ (3-PEt $)_{3}$. A $20 \mathrm{~mL}$ scintillation vial was charged with $\left({ }^{\text {allyl }} \mathrm{CNC}\right) \mathrm{Fe}\left(\mathrm{PEt}_{3}\right)_{2} \quad\left(3-\mathrm{PEt}_{3}\right) \quad(0.019 \mathrm{~g}, 0.033 \mathrm{mmol})$ and $2 \mathrm{~mL}$ benzene. While stirring, 9borabicyclo[3.3.1]nonane $(0.010 \mathrm{~g}, 0.082 \mathrm{mmol})$ was added. The reaction was stirred at room temperature for $16 \mathrm{hr}$. An aliquot was removed and analyzed by NMR spectroscopy. Analysis revealed that all (allyl $\mathrm{CNC}) \mathrm{Fe}\left(\mathrm{PEt}_{3}\right)_{2} \quad\left(3-\mathrm{PEt}_{3}\right)$ was consumed, the major phosphorus containing species was the $\mathrm{PEt}_{3} \rightarrow 9-\mathrm{BBN}$ adduct, and numerous other unidentifiable species were present. Example spectra are provided below.

Attempted hydroboration of (allyl $\mathrm{CNC}$ )Fe(depe) (3-depe). A $20 \mathrm{~mL}$ scintillation vial was charged with (allyl $\mathrm{CNC}$ )Fe(depe) (3-depe) $(0.025 \mathrm{~g}, 0.045 \mathrm{mmol})$ and $4 \mathrm{~mL}$ benzene. While stirring, 9borabicyclo[3.3.1]nonane $(0.012 \mathrm{~g}, 0.098 \mathrm{mmol})$ was added. The reaction was stirred at room temperature for $3 \mathrm{hr}$. An aliquot was removed and analyzed by NMR spectroscopy (longer stirring times resulted in precipitation of insoluble species that were not able to be identified). Analysis revealed that (allyl $\mathrm{CNC}$ )Fe(depe) (3-depe) was not fully consumed and that it was the major phosphorus containing species. The depe $\rightarrow 9-B B N$ adduct and numerous other unidentifiable species were present. Example spectra are provided below.

Attempted hydroboration of $\left({ }^{\text {allyly }} \mathrm{CNC}\right) \mathrm{Fe}\left(\mathrm{CN}^{t} \mathrm{Bu}\right)_{2}\left(4^{-}{ }^{t} \mathrm{Bu}\right)$. A $20 \mathrm{~mL}$ scintillation vial was charged with $\left({ }^{\text {allyl }} \mathrm{CNC}\right) \mathrm{Fe}\left(\mathrm{CN}^{t} \mathrm{Bu}\right)_{2} \quad\left(4^{-}{ }^{t} \mathrm{Bu}\right) \quad(0.015 \mathrm{~g}, \quad 0.029 \mathrm{mmol})$ and $6 \mathrm{~mL}$ toluene. While stirring, 9borabicyclo[3.3.1]nonane $(0.009 \mathrm{~g}, 0.074 \mathrm{mmol})$ was added and the solution stirred at room temperature for $24 \mathrm{hr}$. Volatiles were removed in vacuo. All components soluble in $\mathrm{C}_{6} \mathrm{D}_{6}$ were analyzed by $N M R$ spectroscopy. Analysis revealed multiple species present without evidence of the desired product of latestage hydroboration. While there is evidence of the 1,1-hydroboration byproduct, ${ }^{t} \mathrm{Bu}-\mathrm{N}=\mathrm{CH}(\mathrm{BBN})$, the complexity of the spectrum precludes a definitive identification. Example spectra are provided below. All components insoluble in $\mathrm{C}_{6} \mathrm{D}_{6}$ were further dried, dissolved in THF, and analyzed by NMR spectroscopy. This analysis did not aid in the identification of any byproducts. 


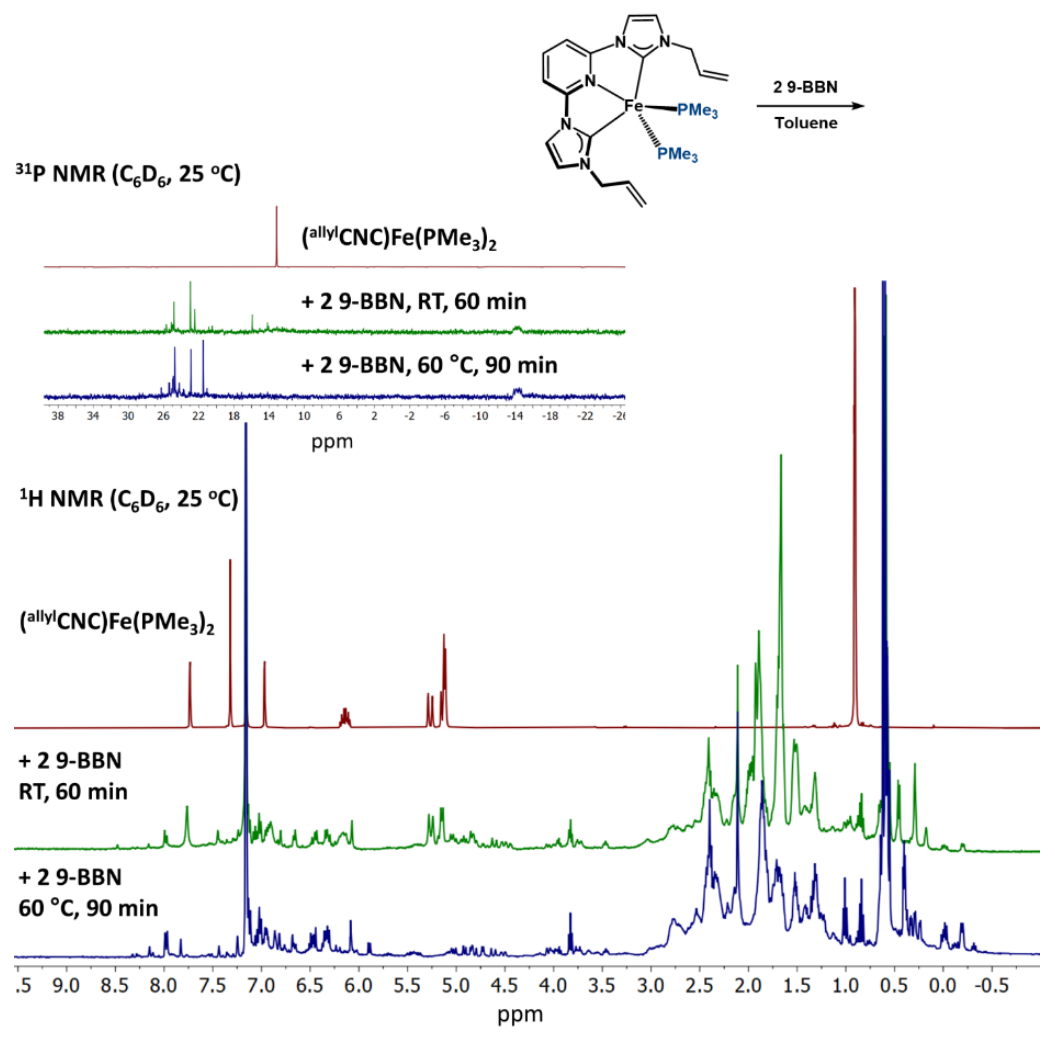

Figure S36 ${ }^{1} \mathrm{H}$ NMR spectra (bottom, $\mathrm{C}_{6} \mathrm{D}_{6}, 25{ }^{\circ} \mathrm{C}, 400 \mathrm{MHz}$ ) and ${ }^{31} \mathrm{P}$ NMR spectra (top, $162 \mathrm{MHz}$ ) corresponding to attempted hydroboration of $\left({ }^{\text {allyl }} \mathrm{CNC}\right) \mathrm{Fe}\left(\mathrm{PMe}_{3}\right)_{2}\left(3-\mathrm{PMe}_{3}\right)$ with 9-BBN.

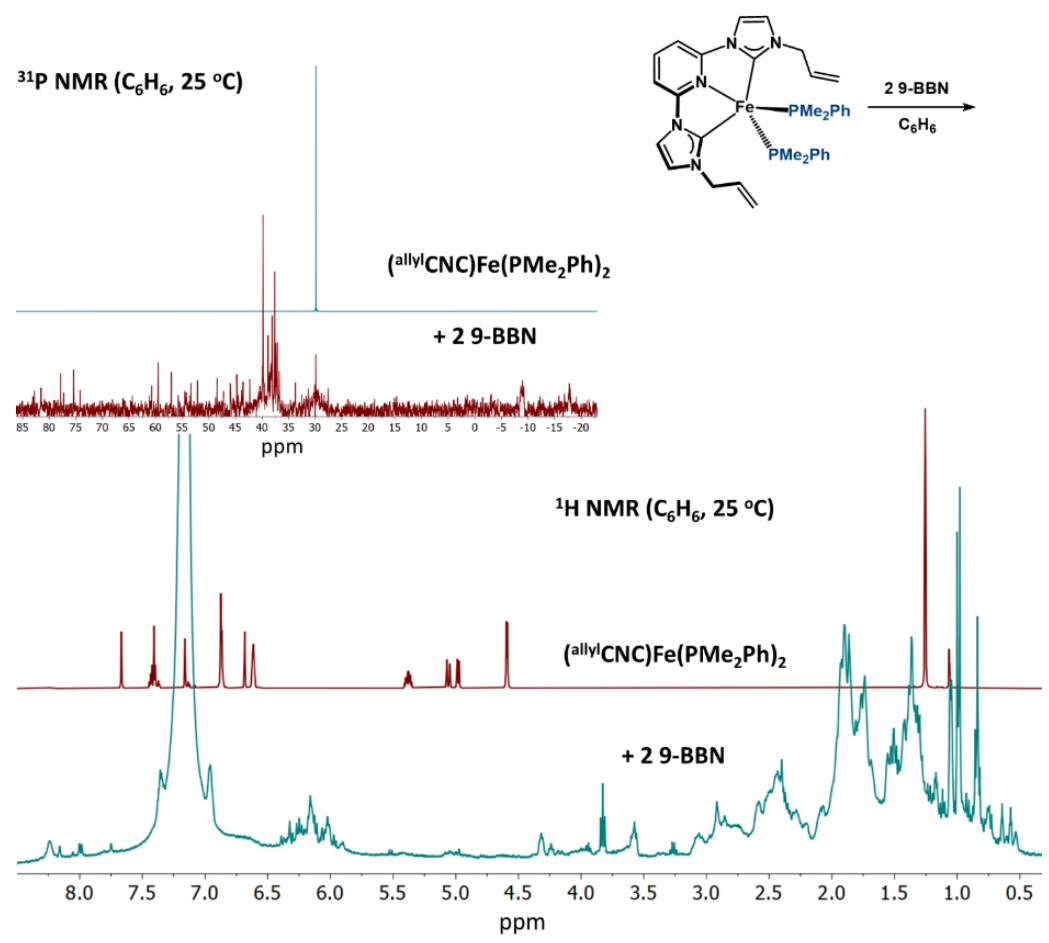

Figure S37 ${ }^{1} \mathrm{H}$ NMR spectra (bottom, $\mathrm{C}_{6} \mathrm{D}_{6}$ and $\mathrm{C}_{6} \mathrm{H}_{6}, 25{ }^{\circ} \mathrm{C}, 400 \mathrm{MHz}$ ) and ${ }^{31} \mathrm{P}$ NMR spectra (top, $162 \mathrm{MHz}$ ) corresponding to attempted hydroboration of (allyl $\mathrm{CNC}) \mathrm{Fe}\left(\mathrm{PMe}_{2} \mathrm{Ph}\right)_{2}\left(3-\mathrm{PMe}_{2} \mathrm{Ph}\right)$ with 9-BBN. 


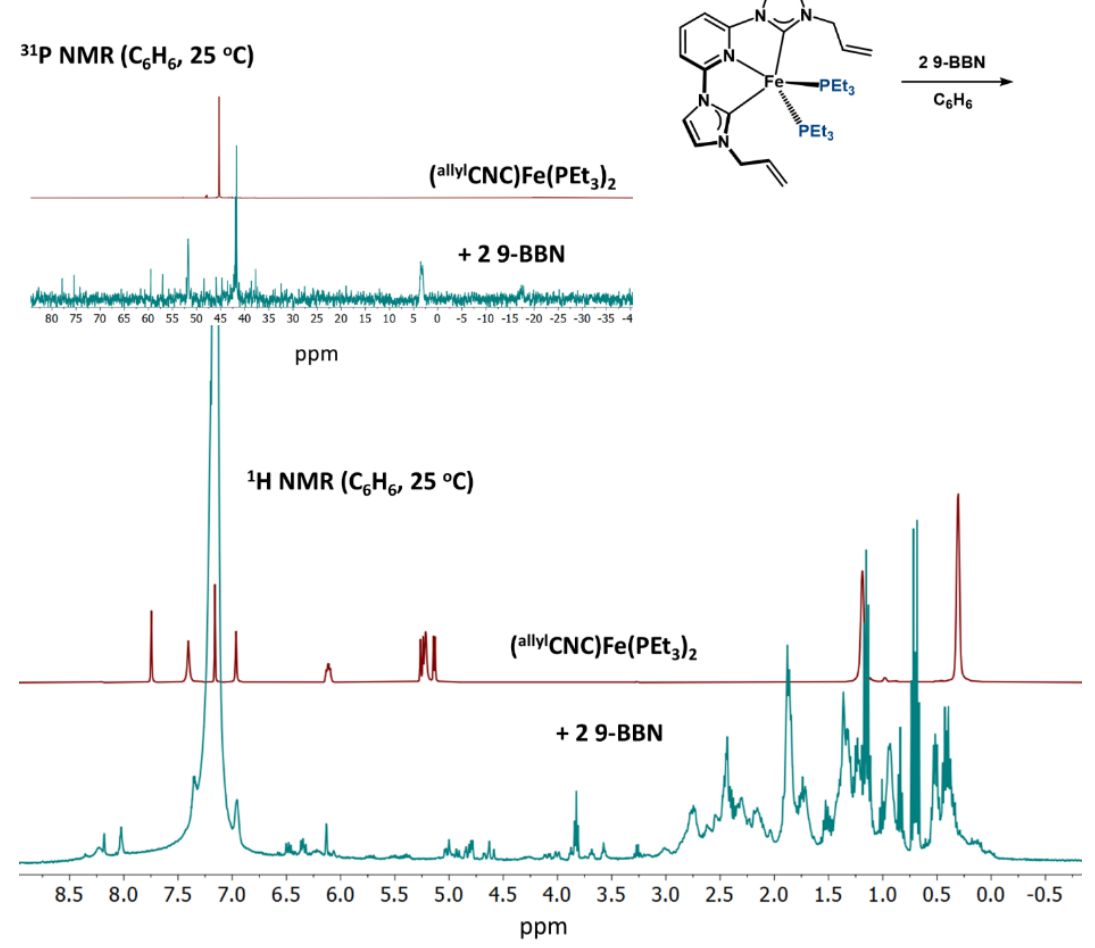

Figure S38 ${ }^{1} \mathrm{H}$ NMR spectra (bottom, $\mathrm{C}_{6} \mathrm{D}_{6}$ and $\mathrm{C}_{6} \mathrm{H}_{6}, 25{ }^{\circ} \mathrm{C}, 400 \mathrm{MHz}$ ) and ${ }^{31} \mathrm{P}$ NMR spectra (top, $162 \mathrm{MHz}$ ) corresponding to attempted hydroboration of $\left({ }^{a l l y l} \mathrm{CNC}\right) \mathrm{Fe}\left(\mathrm{PEt}_{3}\right)_{2}\left(3-\mathrm{PEt}_{3}\right)$ with 9-BBN.

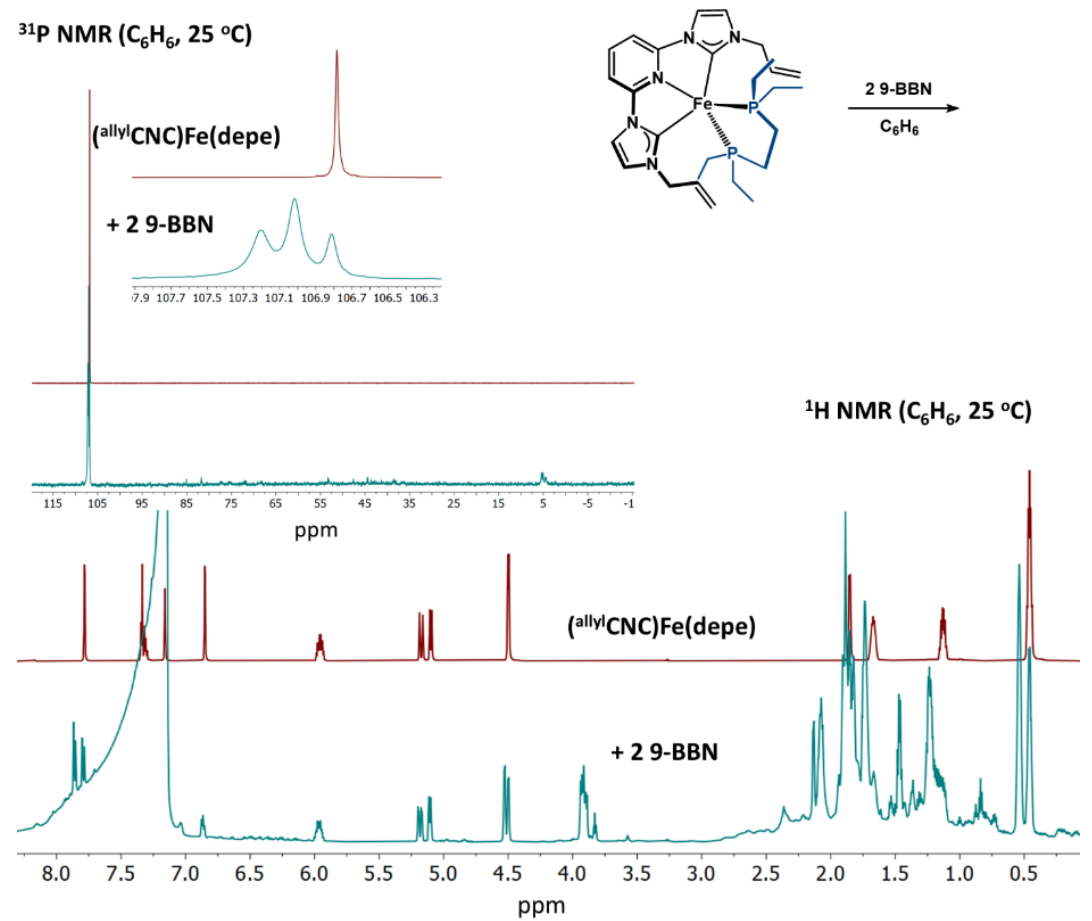

Figure S39 ${ }^{1} \mathrm{H}$ NMR spectra (bottom, $\mathrm{C}_{6} \mathrm{D}_{6}$ and $\mathrm{C}_{6} \mathrm{H}_{6}, 25{ }^{\circ} \mathrm{C}, 700 \mathrm{MHz}$ ) and ${ }^{31} \mathrm{P}$ NMR spectra (top, $283 \mathrm{MHz}$ ) corresponding to attempted hydroboration of (allyl $\mathrm{CNC}) \mathrm{Fe}$ (depe) (3-depe) with 9-BBN. 


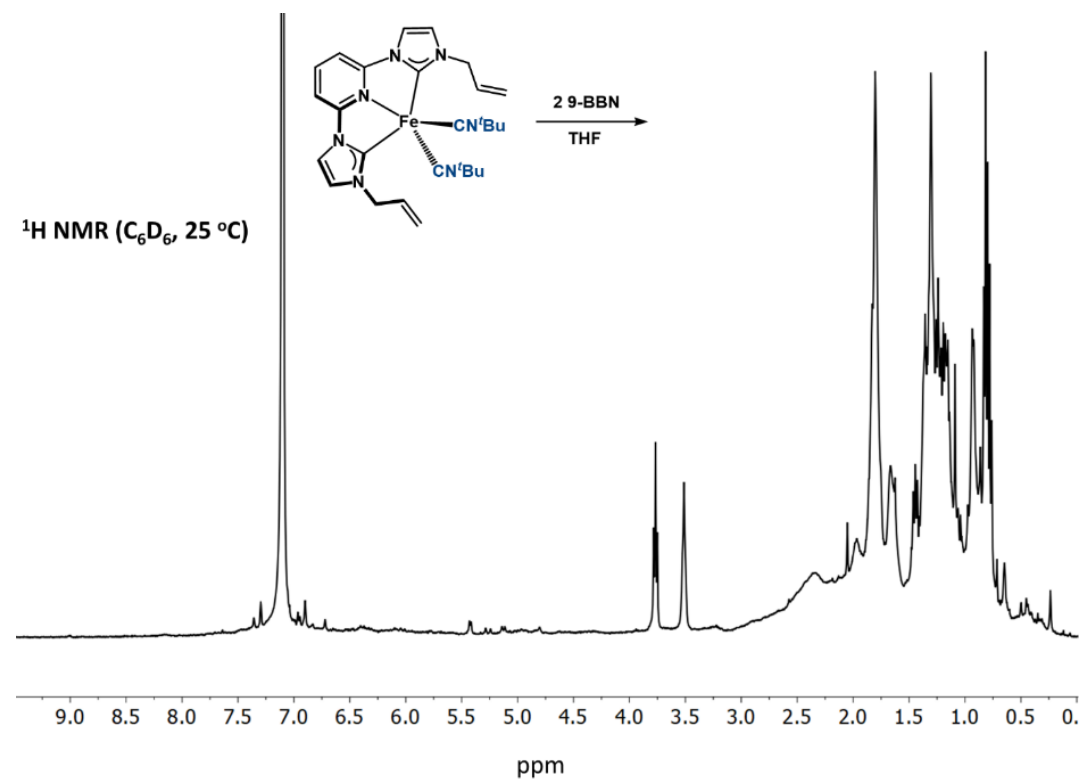

Figure S40 ${ }^{1} \mathrm{H}$ NMR spectrum $\left(\mathrm{C}_{6} \mathrm{D}_{6}, 25{ }^{\circ} \mathrm{C}, 400 \mathrm{MHz}\right)$ corresponding to attempted hydroboration of $\left({ }^{\text {allylyl }} \mathrm{CNC}\right) \mathrm{Fe}\left(\mathrm{CN}^{t} \mathrm{Bu}\right)_{2}\left(4-{ }^{\mathrm{t}} \mathrm{Bu}\right)$ with 9-BBN. 


\section{Acid/base adduct formation}

a) $\mathbf{P R}_{3}+$ 9-borabicyclo[3.3.1]nonane. Stock solutions of 9-borabicyclo[3.3.1]nonane (0.410 M) and each phosphine (solutions ranged $0.213-0.483 \mathrm{M}$ ) were prepared in $\mathrm{C}_{6} \mathrm{D}_{6}$. A $0.100 \mathrm{~mL}$ aliquot of 9-borabicyclo[3.3.1]nonane (4.1 $\mathrm{x}$ $10^{-5} \mathrm{~mol}$ ) was diluted with $0.700 \mathrm{~mL} \mathrm{C}_{6} \mathrm{D}_{6}$. To this solution, the phosphine (0.9 equivalents) was added. After $1 \mathrm{hr}$, the solution was investigated by NMR spectroscopy. Each combination revealed data that is consistent with Lewis acid/base adduct formation. Crude spectra are provided below. Select NMR resonances are provided. $\mathrm{PMe}_{3}:{ }^{1} \mathrm{H} \mathrm{NMR}$ $\left(400 \mathrm{MHz}, \mathrm{C}_{6} \mathrm{D}_{6}\right) \delta=0.61\left(\mathrm{~d}, J=9.4 \mathrm{~Hz}, 9 \mathrm{H}, \mathrm{P}\left(\mathrm{CH}_{3}\right)_{3}\right) .{ }^{31} \mathrm{P} \mathrm{NMR}\left(162 \mathrm{MHz}, \mathrm{C}_{6} \mathrm{D}_{6}\right) \delta=-13.67(\mathrm{~m}) .{ }^{11} \mathrm{~B} \mathrm{NMR}(128 \mathrm{MHz}$, $\left.\mathrm{C}_{6} \mathrm{D}_{6}\right) \delta=-14.30(\mathrm{q}, J=47.2 \mathrm{~Hz}) . \mathrm{PEt}_{3}:{ }^{1} \mathrm{H}$ NMR $\left(400 \mathrm{MHz}, \mathrm{C}_{6} \mathrm{D}_{6}\right) \delta=0.98\left(\mathrm{dt}, J=14.8,7.6 \mathrm{~Hz}, 9 \mathrm{H}, \mathrm{P}\left(\mathrm{CH}_{2} \mathrm{CH}_{3}\right)_{3}\right), 1.22(\mathrm{q}$, $\left.J=7.7 \mathrm{~Hz}, 6 \mathrm{H}, \mathrm{P}\left(\mathrm{CH}_{2} \mathrm{CH}_{3}\right)_{3}\right) .{ }^{31} \mathrm{P} \mathrm{NMR}\left(162 \mathrm{MHz}, \mathrm{C}_{6} \mathrm{D}_{6}\right) \delta=3.51(\mathrm{~m}) .{ }^{11} \mathrm{~B} \mathrm{NMR}\left(128 \mathrm{MHz}, \mathrm{C}_{6} \mathrm{D}_{6}\right) \delta=-16.06(\mathrm{~m}) . \mathrm{PMe} \mathrm{P}_{2} \mathrm{Ph}:$ ${ }^{1} \mathrm{H} N M R\left(400 \mathrm{MHz}, \mathrm{C}_{6} \mathrm{D}_{6}\right) \delta=0.99\left(\mathrm{~d}, J=9.1 \mathrm{~Hz}, 6 \mathrm{H}, \mathrm{P}\left(\mathrm{CH}_{3}\right)_{2} \mathrm{Ph}\right) .{ }^{31} \mathrm{P} \mathrm{NMR}\left(162 \mathrm{MHz}, \mathrm{C}_{6} \mathrm{D}_{6}\right) \delta=-8.90(\mathrm{~m}) .{ }^{11} \mathrm{~B}$ NMR (128 $\left.\mathrm{MHz}, \mathrm{C}_{6} \mathrm{D}_{6}\right) \delta=-14.35(\mathrm{~m})$. depe: ${ }^{31} \mathrm{P}$ NMR $\left(162 \mathrm{MHz}, \mathrm{C}_{6} \mathrm{D}_{6}\right) \delta=4.02$ (broad), 4.95 (broad). ${ }^{11} \mathrm{~B}$ NMR (128 MHz, $\left.\mathrm{C}_{6} \mathrm{D}_{6}\right)$ $\delta=-15.69$ (broad).

b) $\mathbf{P R}_{3}+\mathbf{P h}\left(\mathrm{CH}_{2}\right)_{2} \mathbf{B B N}$. Stock solutions of $\mathrm{PhCH}_{2} \mathrm{CH}_{2} \mathrm{BBN}(0.230 \mathrm{M})$ and each phosphine (solutions ranged $0.213-$ $0.483 \mathrm{M})$ were prepared in $\mathrm{C}_{6} \mathrm{D}_{6}$. A $0.100 \mathrm{~mL}$ aliquot of $\mathrm{PhCH}_{2} \mathrm{CH}_{2} \mathrm{BBN}\left(2.3 \times 10^{-5}\right.$ mol) was diluted with $0.700 \mathrm{~mL}$ $\mathrm{C}_{6} \mathrm{D}_{6}$. To this solution, the phosphine (0.9 equivalents) was added. After $1 \mathrm{hr}$, the solution was investigated by NMR spectroscopy. Each combination revealed data that is consistent with Lewis acid/base adduct formation. Spectra are provided below. Crude spectra are provided below. Select NMR resonances are provided. $\mathrm{PMe}_{3}:{ }^{1} \mathrm{H} \mathrm{NMR}(400 \mathrm{MHz}$, $\left.\mathrm{C}_{6} \mathrm{D}_{6}\right) \delta=0.64\left(\mathrm{~d}, J=8.0 \mathrm{~Hz}, 9 \mathrm{H}, \mathrm{P}\left(\mathrm{CH}_{3}\right)_{3}\right) .{ }^{31} \mathrm{P} \mathrm{NMR}\left(162 \mathrm{MHz}, \mathrm{C}_{6} \mathrm{D}_{6}\right) \delta=-16.73 .{ }^{11} \mathrm{~B} \mathrm{NMR}\left(128 \mathrm{MHz}, \mathrm{C}_{6} \mathrm{D}_{6}\right) \delta=0.09$ (broad). PEt $_{3}:{ }^{1} \mathrm{H}$ NMR $\left(400 \mathrm{MHz}, \mathrm{C}_{6} \mathrm{D}_{6}\right) \delta=0.88\left(\mathrm{dt}, J=13.5,7.7 \mathrm{~Hz}, 9 \mathrm{H}, \mathrm{P}\left(\mathrm{CH}_{2} \mathrm{CH}_{3}\right)_{3}\right), 1.25$ (qd, $\left.J=7.7,2.3 \mathrm{~Hz}, 6 \mathrm{H}, \mathrm{P}\left(\mathrm{CH}_{2} \mathrm{CH}_{3}\right)_{3}\right)$. ${ }^{31} \mathrm{P}\left\{{ }^{1} \mathrm{H}\right\}$ NMR $\left(162 \mathrm{MHz}, \mathrm{C}_{6} \mathrm{D}_{6}\right) \delta=-13.36 .{ }^{11} \mathrm{~B} \mathrm{NMR}\left(128 \mathrm{MHz}, \mathrm{C}_{6} \mathrm{D}_{6}\right) \delta=49.18$. $\mathrm{PMe}_{2} \mathrm{Ph}:{ }^{1} \mathrm{H}$ NMR $\left(400 \mathrm{MHz}, \mathrm{C}_{6} \mathrm{D}_{6}\right) \delta=$ $\left.1.04\left(\mathrm{~d}, J=2.6 \mathrm{~Hz}, 6 \mathrm{H}, \mathrm{P}\left(\mathrm{CH}_{3}\right)_{2} \mathrm{Ph}\right)\right) .{ }^{31} \mathrm{P}\left\{{ }^{1} \mathrm{H}\right\} \mathrm{NMR}\left(162 \mathrm{MHz}, \mathrm{C}_{6} \mathrm{D}_{6}\right) \delta=-27.01 .{ }^{11} \mathrm{~B} \mathrm{NMR}\left(128 \mathrm{MHz}, \mathrm{C}_{6} \mathrm{D}_{6}\right) \delta=20.89$. depe: ${ }^{1} \mathrm{H}$ NMR $\left(400 \mathrm{MHz}, \mathrm{C}_{6} \mathrm{D}_{6}\right) \delta=0.93\left(\mathrm{dt}, J=14.8,7.6 \mathrm{~Hz}, 9 \mathrm{H}, \mathrm{P}\left(\mathrm{CH}_{2} \mathrm{CH}_{3}\right)_{3}\right), 1.27\left(\mathrm{q}, J=7.7 \mathrm{~Hz}, 6 \mathrm{H}, \mathrm{P}\left(\mathrm{CH}_{2} \mathrm{CH}_{3}\right)_{3}\right)$. ${ }^{31} \mathrm{P}\left\{{ }^{1} \mathrm{H}\right\}$ NMR (162 MHz, $\left.\mathrm{C}_{6} \mathrm{D}_{6}\right) \delta=-15.05 .{ }^{11} \mathrm{~B} \mathrm{NMR}\left(128 \mathrm{MHz}, \mathrm{C}_{6} \mathrm{D}_{6}\right) \delta=67.73$.

c) CNR + 9-borabicyclo[3.3.1]nonane. Stock solutions of 9-borabicyclo[3.3.1]nonane (0.410 M) and each isocyanide (solutions ranged $0.166-0.442 \mathrm{M}$ ) were prepared in $\mathrm{C}_{6} \mathrm{D}_{6}$. A $0.100 \mathrm{~mL}$ aliquot of 9-borabicyclo[3.3.1]nonane (4.1 $\mathrm{x}$ $10^{-5} \mathrm{~mol}$ ) was diluted with $0.700 \mathrm{~mL} \mathrm{C}_{6} \mathrm{D}_{6}$. To this solution, the isocyanide ( 0.9 equivalents) was added. After $1 \mathrm{hr}$, the solution was investigated by NMR spectroscopy. Each combination revealed data that is consistent with Lewis acid/base adduct formation and/or 1,1-hydroboration of the isocyanide. Crude spectra are provided below. Select NMR resonances are provided. $\mathrm{CN}^{t} \mathrm{Bu}:{ }^{1} \mathrm{H}$ NMR $\left(400 \mathrm{MHz}, \mathrm{C}_{6} \mathrm{D}_{6}\right) \delta=0.80\left(\mathrm{~s}, 9 \mathrm{H}, \mathrm{C}\left(\mathrm{CH}_{3}\right)_{3}\right.$, acid/base adduct), 1.34 (s, $9 \mathrm{H},=\mathrm{NC}\left(\mathrm{CH}_{3}\right)_{3}, 1,1$-hydroboration), $9.04\left(\mathrm{~s}, 1 \mathrm{H}, \mathrm{N}=\mathrm{CH}(\mathrm{BBN}), 1\right.$,1-hydroboration). ${ }^{11} \mathrm{~B} \mathrm{NMR}\left(128 \mathrm{MHz}, \mathrm{C}_{6} \mathrm{D}_{6}\right) \delta=-15.89$ (acid/base adduct). CNPh: ${ }^{1} \mathrm{H}$ NMR (400 MHz, $\left.\mathrm{C}_{6} \mathrm{D}_{6}\right) \delta=9.30$ (s, 1H, N=CH(BBN), 1,1-hydroboration). ${ }^{11} \mathrm{~B}$ NMR (128 $\left.\mathrm{MHz}, \mathrm{C}_{6} \mathrm{D}_{6}\right) \delta=-15.38$ (acid/base adduct). $\mathrm{CN}^{t} \mathrm{Bu}_{3} \mathrm{C}_{6} \mathrm{H}_{2}$ : (the acid/base adduct and 1,1-hydroboration product form in 1:1 ratio; the NMR assignments are unclear). ${ }^{1} \mathrm{H} \mathrm{NMR}\left(400 \mathrm{MHz}, \mathrm{C}_{6} \mathrm{D}_{6}\right) \delta=1.12\left(\mathrm{~s}, 9 \mathrm{H}, p-\mathrm{C}\left(\mathrm{CH}_{3}\right)_{3}\right), 1.40(\mathrm{~s}, 9 \mathrm{H}, p-$ $\left.\mathrm{C}\left(\mathrm{CH}_{3}\right)_{3}\right), 1.53\left(\mathrm{~s}, 18 \mathrm{H}, \mathrm{o}-\mathrm{C}\left(\mathrm{CH}_{3}\right)_{3}\right), 1.60\left(\mathrm{~s}, 18 \mathrm{H}, \mathrm{o}-\mathrm{C}\left(\mathrm{CH}_{3}\right)_{3}\right), 7.40(\mathrm{~s}, 2 \mathrm{H}, \mathrm{m}-\mathrm{Ph}-\mathrm{CH}), 7.58(\mathrm{~s}, 2 \mathrm{H}, \mathrm{m}-\mathrm{Ph}-\mathrm{CH}), 8.84(\mathrm{~s}, 1 \mathrm{H}$, $\mathrm{N}=\mathrm{CH}(\mathrm{BBN}), 1,1$-hydroboration). ${ }^{11} \mathrm{~B} N \mathrm{NMR}\left(128 \mathrm{MHz}, \mathrm{C}_{6} \mathrm{D}_{6}\right) \delta=-15.08$ (acid/base adduct).

d) $\mathrm{CNR}+\mathrm{Ph}\left(\mathrm{CH}_{2}\right)_{2} \mathrm{BBN}$. Stock solutions of $\mathrm{PhCH}_{2} \mathrm{CH}_{2} \mathrm{BBN}(0.230 \mathrm{M})$ and each isocyanide (solutions ranged $0.166-$ $0.442 \mathrm{M})$ were prepared in $\mathrm{C}_{6} \mathrm{D}_{6}$. A $0.100 \mathrm{~mL}$ aliquot of $\mathrm{PhCH}_{2} \mathrm{CH}_{2} \mathrm{BBN}\left(2.3 \times 10^{-5}\right.$ mol) was diluted with $0.700 \mathrm{~mL}$ $\mathrm{C}_{6} \mathrm{D}_{6}$. To this solution, the isocyanide ( 0.9 equivalents) was added. After $1 \mathrm{hr}$, the solution was investigated by NMR spectroscopy. Each combination revealed data that is consistent with Lewis acid/base adduct formation. Crude spectra are provided below. Select NMR resonances are provided. $\mathrm{CN}^{t} \mathrm{Bu}:{ }^{1} \mathrm{H} N \mathrm{NMR}\left(400 \mathrm{MHz}, \mathrm{C}_{6} \mathrm{D}_{6}\right) \delta=0.68(\mathrm{~s}, 9 \mathrm{H}$, $\left.\mathrm{C}\left(\mathrm{CH}_{3}\right)_{3}\right) .{ }^{11} \mathrm{~B}$ NMR (128 MHz, $\left.\mathrm{C}_{6} \mathrm{D}_{6}\right) \delta=-17.28$ (acid/base adduct). CNPh: ${ }^{11} \mathrm{~B}$ NMR $\left(128 \mathrm{MHz}, \mathrm{C}_{6} \mathrm{D}_{6}\right) \delta=-16.50$ (acid/base adduct). $\mathrm{CN}^{t} \mathrm{Bu}_{3} \mathrm{C}_{6} \mathrm{H}_{2}:{ }^{1} \mathrm{H}$ NMR $\left(400 \mathrm{MHz}, \mathrm{C}_{6} \mathrm{D}_{6}\right) \delta=1.17\left(\mathrm{~s}, 9 \mathrm{H}, p-\mathrm{C}\left(\mathrm{CH}_{3}\right)_{3}\right), 1.40\left(\mathrm{~s}, 18 \mathrm{H}, o-\mathrm{C}\left(\mathrm{CH}_{3}\right)_{3}\right), 7.37(\mathrm{~s}$, $2 \mathrm{H}, \mathrm{m}-\mathrm{Ph}-\mathrm{CH}) .{ }^{11} \mathrm{~B} N \mathrm{NMR}\left(128 \mathrm{MHz}, \mathrm{C}_{6} \mathrm{D}_{6}\right) \delta=-15.62$ (acid/base adduct). 


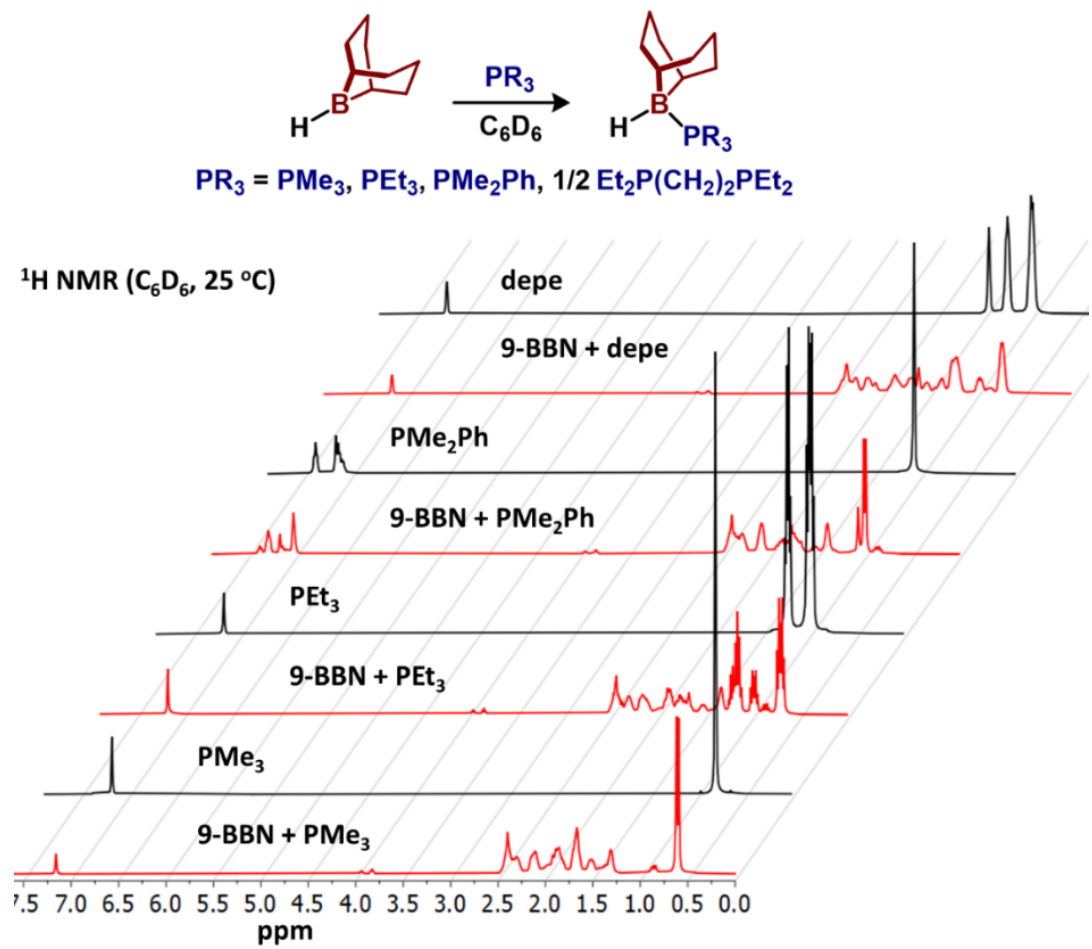

Figure S41 ${ }^{1} \mathrm{H}$ NMR spectra $\left(\mathrm{C}_{6} \mathrm{D}_{6}, 25{ }^{\circ} \mathrm{C}, 400 \mathrm{MHz}\right)$ corresponding to reaction between 9-BBN and $\mathrm{PR}_{3}$ (red). Authentic ${ }^{1} \mathrm{H}$ NMR spectra of $\mathrm{PR}_{3}$ are provided (black).

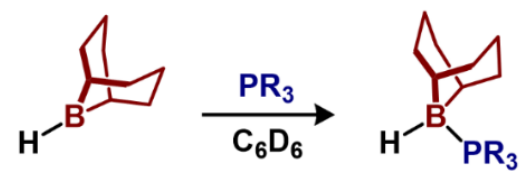

$$
\mathrm{PR}_{3}=\mathrm{PMe}_{3}, \mathrm{PEt}_{3}, \mathrm{PMe}_{2} \mathrm{Ph}, 1 / 2 \mathrm{Et}_{2} \mathrm{P}\left(\mathrm{CH}_{2}\right)_{2} \mathrm{PEt}_{2}
$$

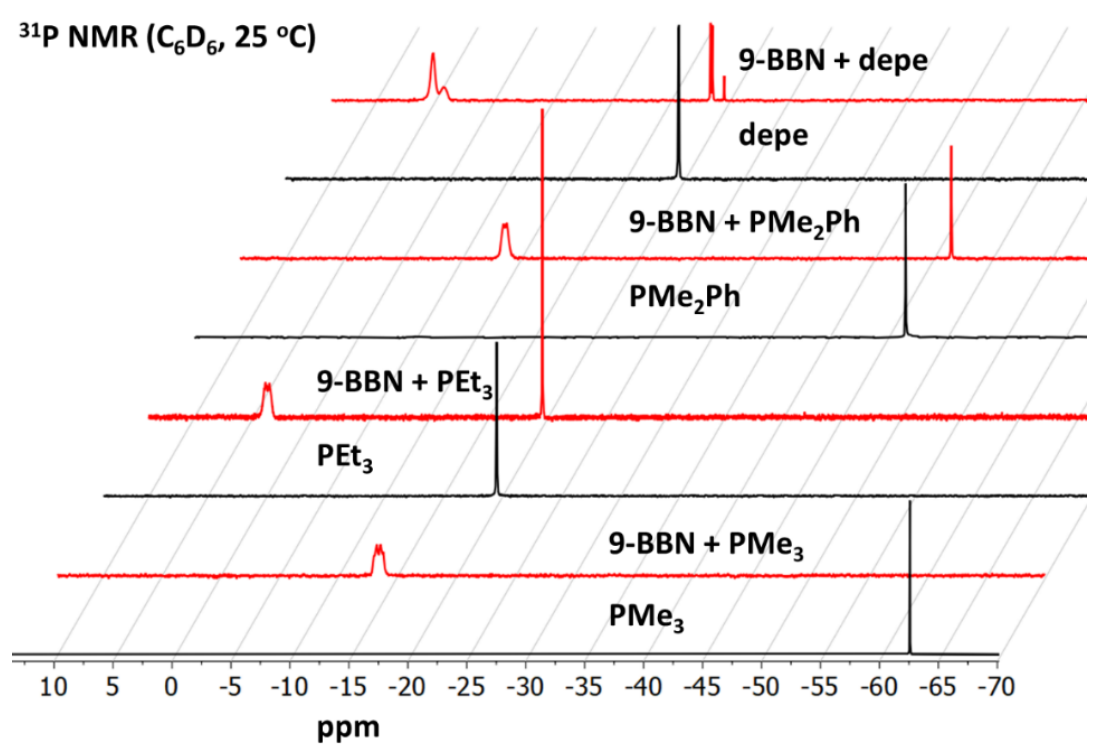

Figure S42 ${ }^{31} \mathrm{P}$ NMR spectra $\left(\mathrm{C}_{6} \mathrm{D}_{6}, 25{ }^{\circ} \mathrm{C}, 202 \mathrm{MHz}\right)$ corresponding to reaction between 9-BBN and $\mathrm{PR}_{3}$ (red). Authentic ${ }^{31} \mathrm{P}$ NMR spectra of $\mathrm{PR}_{3}$ are provided (black). 

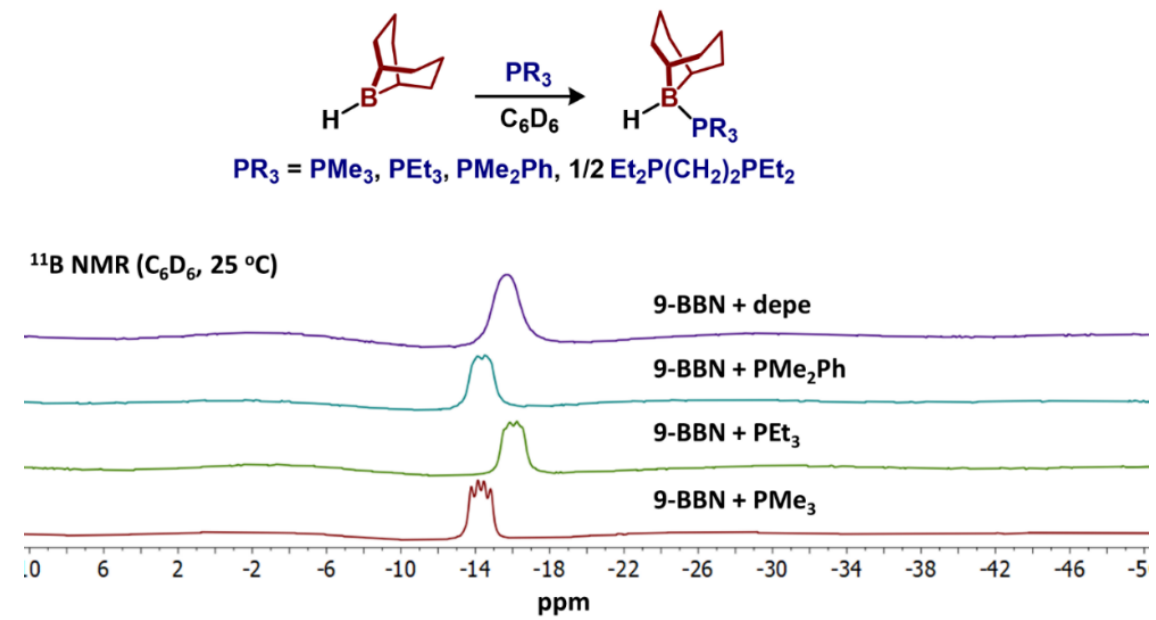

Figure $\mathrm{S} 43{ }^{11} \mathrm{~B}$ NMR spectra $\left(\mathrm{C}_{6} \mathrm{D}_{6}, 25{ }^{\circ} \mathrm{C}, 128 \mathrm{MHz}\right)$ corresponding to reaction between 9-BBN and $\mathrm{PR}_{3}$.

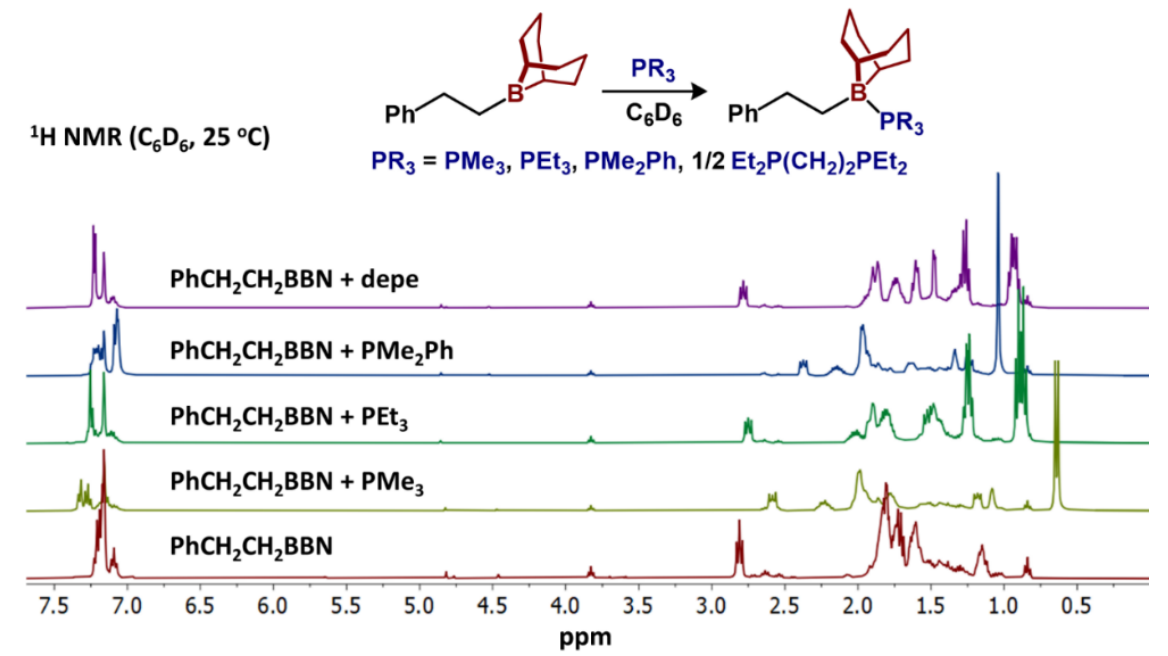

Figure $\mathrm{S44}{ }^{1} \mathrm{H}$ NMR spectra $\left(\mathrm{C}_{6} \mathrm{D}_{6}, 25{ }^{\circ} \mathrm{C}, 400 \mathrm{MHz}\right)$ corresponding to reaction between $\mathrm{Ph}\left(\mathrm{CH}_{2}\right)_{2} \mathrm{BBN}$ and $\mathrm{PR}_{3}$. 


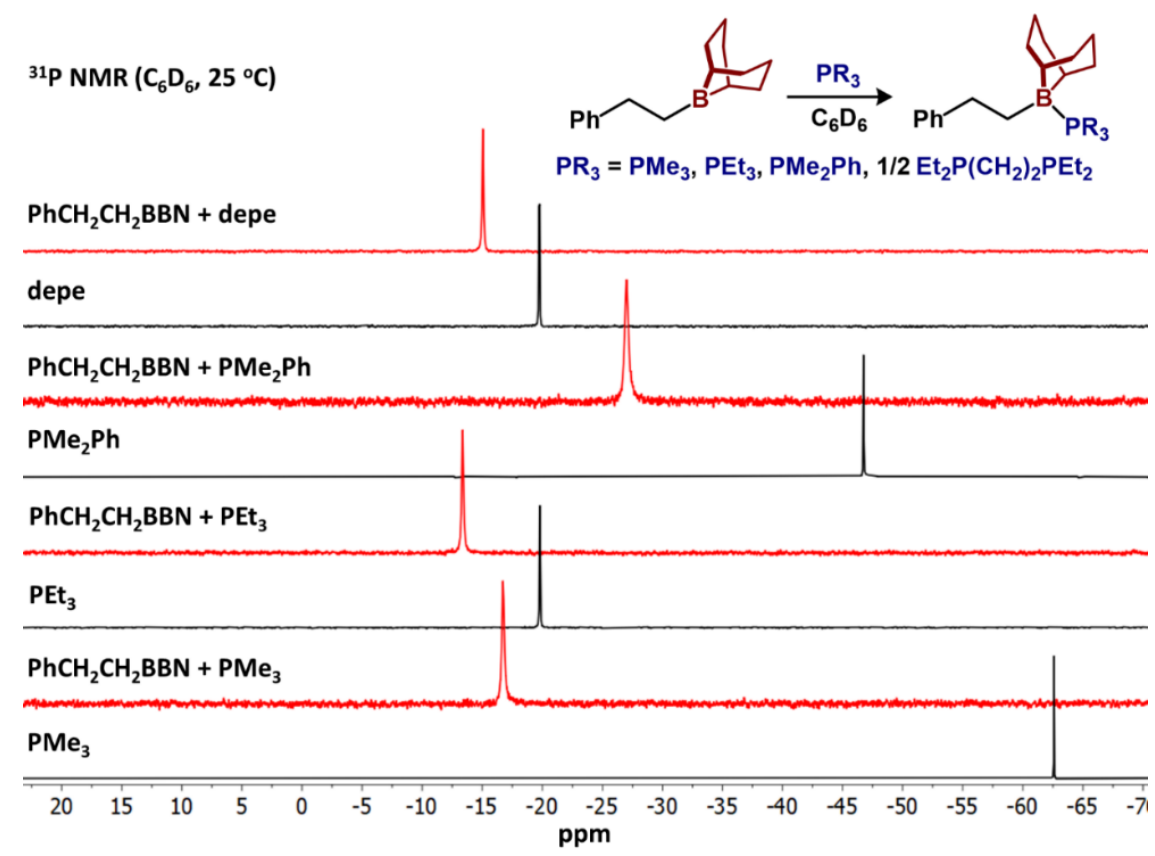

Figure $\mathrm{S45}{ }^{31} \mathrm{P}$ NMR spectra $\left(\mathrm{C}_{6} \mathrm{D}_{6}, 25{ }^{\circ} \mathrm{C}, 202 \mathrm{MHz}\right)$ corresponding to reaction between $\mathrm{Ph}\left(\mathrm{CH}_{2}\right)_{2} \mathrm{BBN}$ and $\mathrm{PR}_{3}$ (red). Authentic ${ }^{31} \mathrm{P} N M R$ spectra of $\mathrm{PR}_{3}$ are provided (black).

${ }^{11} B$ NMR $\left(C_{6} D_{6}, 25^{\circ} \mathrm{C}\right)$

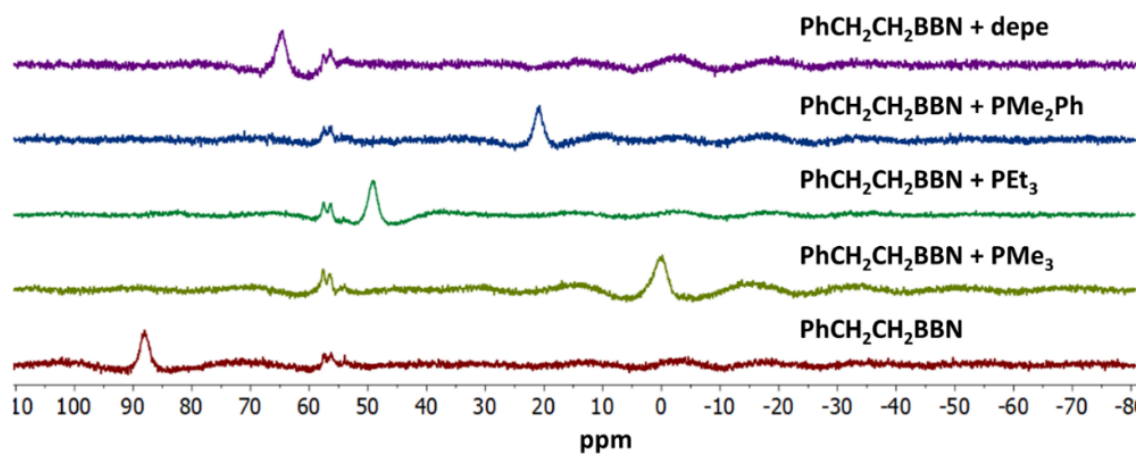

Figure S46 ${ }^{11} \mathrm{~B}$ NMR spectra $\left(\mathrm{C}_{6} \mathrm{D}_{6}, 25{ }^{\circ} \mathrm{C}, 128 \mathrm{MHz}\right)$ corresponding to reaction between $\mathrm{Ph}\left(\mathrm{CH}_{2}\right)_{2} \mathrm{BBN}$ and $\mathrm{PR}_{3}$. 


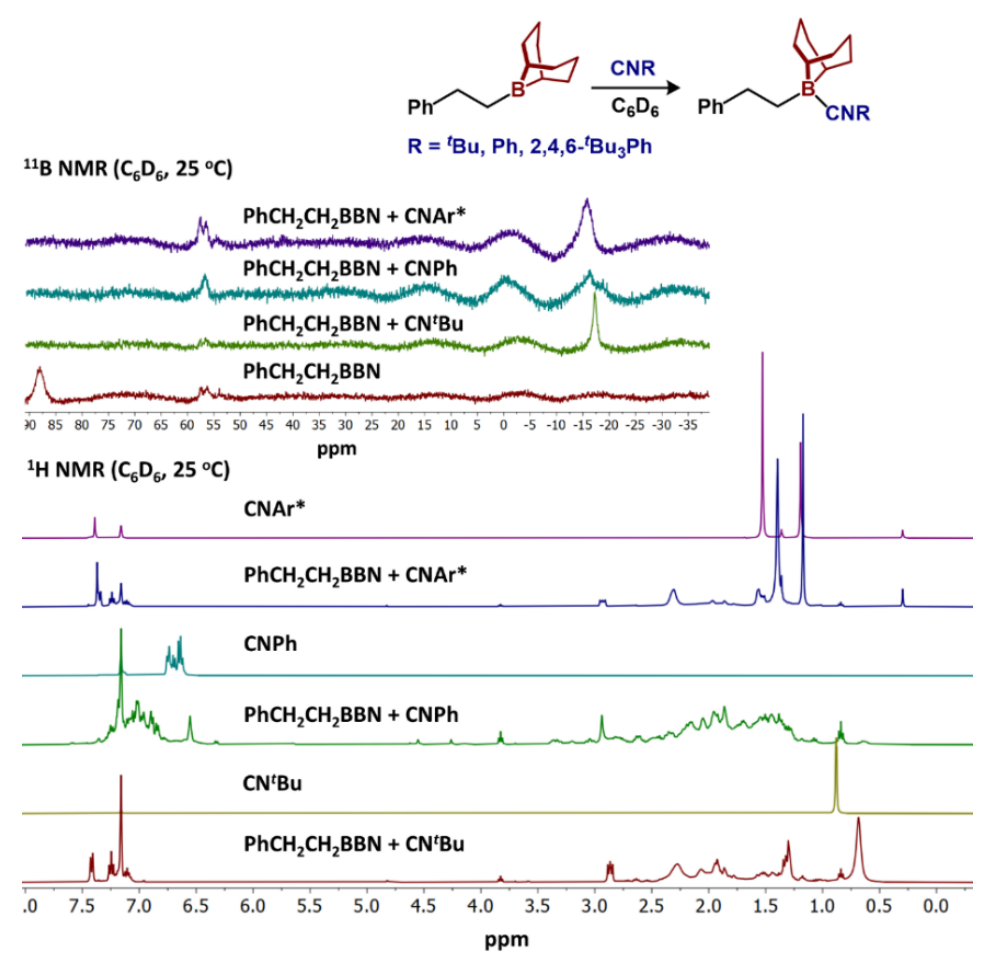

Figure S47 ${ }^{1} \mathrm{H}$ NMR spectra (bottom, $\mathrm{C}_{6} \mathrm{D}_{6}, 25{ }^{\circ} \mathrm{C}, 400 \mathrm{MHz}$ ) and ${ }^{11} \mathrm{~B} \mathrm{NMR}$ spectra (top, $128 \mathrm{MHz}$ ) corresponding to the reactions between $\mathrm{Ph}\left(\mathrm{CH}_{2}\right)_{2} \mathrm{BBN}$ and CNR. Authentic ${ }^{1} \mathrm{H}$ NMR spectra of $\mathrm{CNR}$ are provided.

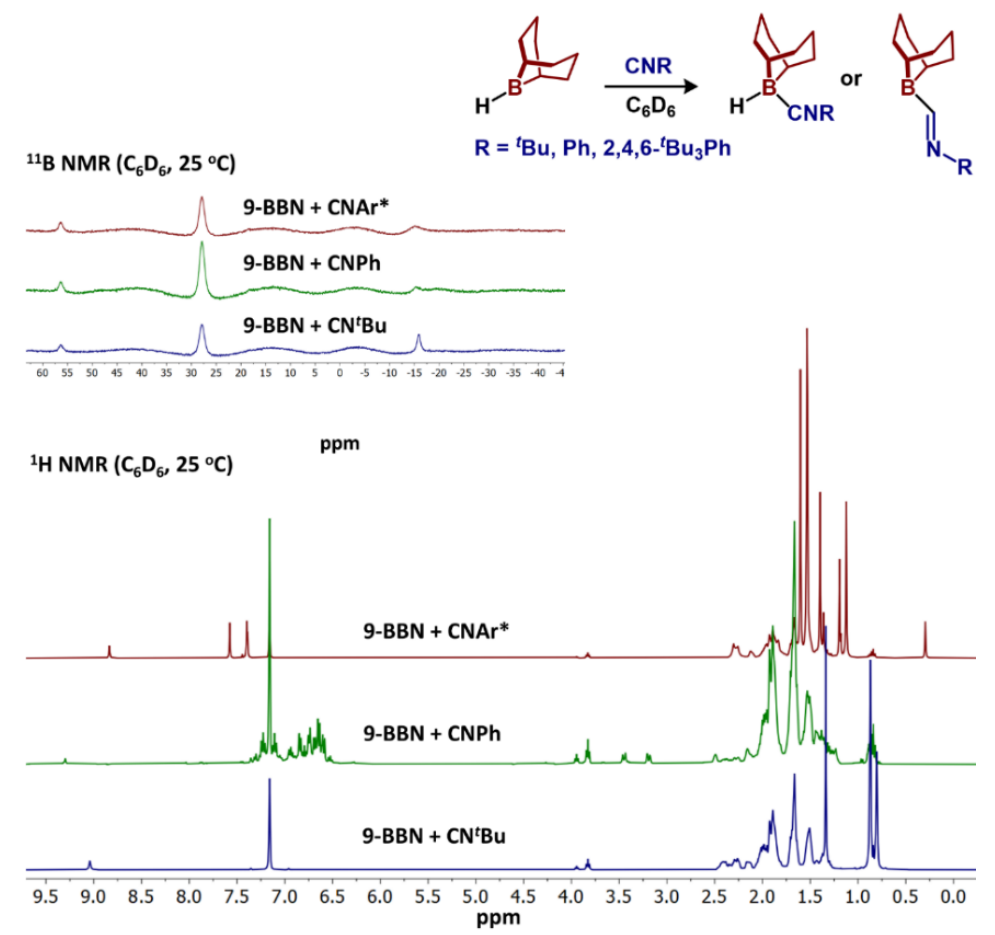

Figure S48 ${ }^{1} \mathrm{H}$ NMR spectra (bottom, $\mathrm{C}_{6} \mathrm{D}_{6}, 25{ }^{\circ} \mathrm{C}, 400 \mathrm{MHz}$ ) and ${ }^{11} \mathrm{~B} \mathrm{NMR}$ spectra (top, $128 \mathrm{MHz}$ ) corresponding to reaction between 9-BBN and CNR. 


\section{Control Reactions involving $\left({ }^{\mathrm{BPin}} \mathrm{CNC}\right) \mathrm{Fe}\left(\mathrm{PMe}_{3}\right)_{2}\left(6-\mathrm{PMe}_{3}\right)$.}

Stability of ( $\left.{ }^{\mathrm{BPin}} \mathrm{CNC}\right) \mathrm{Fe}\left(\mathrm{PMe}_{3}\right)_{2}$ (6-PMe $\left.{ }_{3}\right)$ in cyclohexane. In a $\mathrm{N}_{2}$ filled glovebox, a J-Young NMR tube was charged with $0.600 \mathrm{~mL}$ of a $1.0326 \times 10^{-3} \mathrm{M}$ stock solution of $\left({ }^{\mathrm{BPin}} \mathrm{CNC}\right) \mathrm{Fe}\left(\mathrm{PMe}_{3}\right)_{2}\left(6-\mathrm{PMe}_{3}\right)$ in cyclohexane. The tube was sealed and initial ${ }^{1} \mathrm{H}$ and ${ }^{31} \mathrm{P}$ NMR spectra were obtained. The tube was protected from light and left undisturbed in a $\mathrm{N}_{2}$ glovebox for 6 days. After 6 days, ${ }^{1} \mathrm{H}$ and ${ }^{31} \mathrm{P}$ NMR spectra were obtained and reveal minimal degradation. See spectra below.

\section{Reaction between ( $\left.{ }^{\mathrm{BPin}} \mathrm{CNC}\right) \mathrm{Fe}\left(\mathrm{PMe}_{3}\right)_{2}\left(6-\mathrm{PMe}_{3}\right)$ and $\mathrm{PhCH}_{2} \mathrm{CH}_{2} \mathrm{BBN}$.}

1a) Reaction between one equivalent each of $\left({ }^{\mathrm{BPin}} \mathrm{CNC}\right) \mathrm{Fe}\left(\mathrm{PMe}_{3}\right)_{2}$ (6-PMe $\left.\mathbf{P}_{3}\right)$ and $\mathrm{PhCH}_{2} \mathrm{CH}_{2} \mathrm{BBN}$ in benzene$d_{6}\left(C_{6} D_{6}\right)$ : In a $N_{2}$ filled glovebox, a $20 \mathrm{~mL}$ scintillation vial was charged with $0.600 \mathrm{~mL} \mathrm{C}_{6} \mathrm{D}_{6}$. From a stock solution (6.884 $\times 10^{-3} \mathrm{M}$ in $\left.\mathrm{C}_{6} \mathrm{D}_{6}\right),\left({ }^{\mathrm{BPin}} \mathrm{CNC}\right) \mathrm{Fe}\left(\mathrm{PMe}_{3}\right)_{2}\left(6-\mathrm{PMe}_{3}\right)(0.150 \mathrm{~mL}, 0.001 \mathrm{mmol})$ was added to the vial. From a stock solution (0.2631 $\mathrm{M}$ in $\left.\mathrm{C}_{6} \mathrm{D}_{6}\right), \mathrm{PhCH}_{2} \mathrm{CH}_{2} \mathrm{BBN}(0.004 \mathrm{~mL}, 0.001 \mathrm{mmol})$ was next added to the vial. The contents of the vial were transferred to a J-Young NMR tube and sealed. After $8 \mathrm{hr}$, the NMR tube was removed from the glovebox and ${ }^{1} \mathrm{H}$ and ${ }^{31} \mathrm{P}$ NMR spectra were obtained.

1b) The tube from the aforementioned reaction was then subjected to three freeze-pump-thaw cycles on a Schlenk line, resulting in a final internal pressure of 0.140 Torr. The tube was left attached to the Schlenk line for $16 \mathrm{hr}$, then ${ }^{1} \mathrm{H}$ and ${ }^{31} \mathrm{P}$ NMR spectra were obtained. Representative spectra of the reaction before and after the freeze-pump-thaw cycles are provided below.

2) Reaction between one equivalent each of ( $\left.{ }^{\mathrm{BPin}} \mathrm{CNC}\right) \mathrm{Fe}\left(\mathrm{PMe}_{3}\right)_{2}\left(6-\mathrm{PMe}_{3}\right)$ and $\mathrm{PhCH}_{2} \mathrm{CH}_{2} \mathrm{BBN}$ in benzene$d_{6}\left(\mathrm{C}_{6} \mathrm{D}_{6}\right)$ under static vacuum (0.140 Torr): In a $\mathrm{N}_{2}$ filled glovebox, a $20 \mathrm{~mL}$ scintillation vial was charged

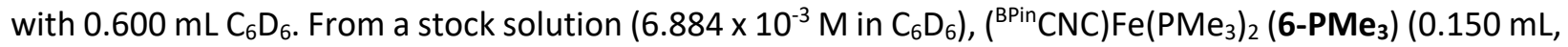

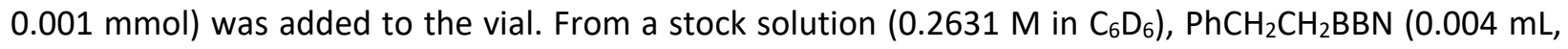
$0.001 \mathrm{mmol}$ ) was next added to the vial. The contents of the vial were transferred to a J-Young NMR tube and sealed. The tube was removed from the glovebox, attached to a Schlenk line, and then subjected to three freeze-pump-thaw cycles, resulting in a final internal pressure of 0.140 Torr. The tube was left attached to the Schlenk line for $8 \mathrm{hr}$, then ${ }^{1} \mathrm{H}$ and ${ }^{31} \mathrm{P}$ NMR spectra were obtained. Representative spectra are provided below.

3) Reaction between $\left({ }^{\mathrm{BPin}} \mathrm{CNC}\right) \mathrm{Fe}\left(\mathrm{PMe}_{3}\right)_{2}\left(6-\mathrm{PMe}_{3}\right)$ and two equivalents $\mathrm{PhCH}_{2} \mathrm{CH}_{2} \mathrm{BBN}$ in benzene- $d_{6}$ $\left(\mathrm{C}_{6} \mathrm{D}_{6}\right)$ : In a $\mathrm{N}_{2}$ filled glovebox, a $20 \mathrm{~mL}$ scintillation vial was charged with $0.200 \mathrm{~mL} \mathrm{C}_{6} \mathrm{D}_{6}$. From a stock solution $\left(6.884 \times 10^{-3} \mathrm{M}\right.$ in $\left.\mathrm{C}_{6} \mathrm{D}_{6}\right),\left({ }^{\mathrm{BPin}} \mathrm{CNC}\right) \mathrm{Fe}\left(\mathrm{PMe}_{3}\right)_{2}\left(6-\mathrm{PMe}_{3}\right)(0.400 \mathrm{~mL}, 0.0028 \mathrm{mmol})$ was added to the vial. From a stock solution (0.2631 $\mathrm{M}$ in $\left.\mathrm{C}_{6} \mathrm{D}_{6}\right), \mathrm{PhCH}_{2} \mathrm{CH}_{2} \mathrm{BBN}(0.021 \mathrm{~mL}, 0.0055 \mathrm{mmol})$ was next added to the vial. The contents of the vial were transferred to a J-Young NMR tube and sealed. After $10 \mathrm{~min},{ }^{1} \mathrm{H}$ and ${ }^{31} \mathrm{P}$ NMR spectra were obtained revealing the presence of three species: $\left({ }^{\mathrm{BPin}} \mathrm{CNC}\right) \mathrm{Fe}\left(\mathrm{PMe}_{3}\right)_{2}\left(6-\mathrm{PMe}_{3}\right)$, $\left({ }^{B P i n} \mathrm{CNC}\right) \mathrm{Fe}\left(\mathrm{PMe}_{3}\right)\left(\mathrm{N}_{2}\right)$, and $\mathrm{Ph}\left(\mathrm{CH}_{2}\right)_{2} \mathrm{BBN}-\mathrm{PMe}_{3}$. Spectra are provided below. The tube was brought back into a glovebox, poured into a $20 \mathrm{~mL}$ scintillation vial, and volatiles were removed in vacuo. The material was combined with dry $\mathrm{KBr}$ and pressed into a pellet. Analysis by infrared spectroscopy is consistent with the presence of an $\mathrm{N}_{2}$ ligand $\left(1975 \mathrm{~cm}^{-1}\right)$. 
4) Reaction between $\left({ }^{\mathrm{BPin}} \mathrm{CNC}\right) \mathrm{Fe}\left(\mathrm{PMe}_{3}\right)_{2}\left(6-\mathrm{PMe}_{3}\right)$ and two equivalents $\mathrm{PhCH}_{2} \mathrm{CH}_{2} \mathrm{BBN}$ in cyclohexane $\left(\mathrm{C}_{6} \mathrm{H}_{12}\right)$ : A $20 \mathrm{~mL}$ scintillation vial was charged with $\left({ }^{\mathrm{BPin}} \mathrm{CNC}\right) \mathrm{Fe}\left(\mathrm{PMe}_{3}\right)_{2}\left(6-\mathrm{PMe}_{3}\right)\left(1.000 \mathrm{~mL}, 1.033 \times 10^{-3} \mathrm{M}\right.$ stock solution in cyclohexane, $0.001 \mathrm{mmol})$ and $\mathrm{PhCH}_{2} \mathrm{CH}_{2} \mathrm{BBN}\left(0.133 \mathrm{~mL}, 1.548 \times 10^{-2} \mathrm{M}\right.$ stock solution in cyclohexane, $0.002 \mathrm{mmol}$ ). The solution was transferred to a J-Young NMR tube and analysis by NMR spectroscopy was performed after 30 min of mixing. Authentic NMR spectra of both starting materials (stock solution concentration) were also obtained for comparison. The ${ }^{1} \mathrm{H}$ NMR analysis is consistent with formation of $\left({ }^{\mathrm{BPin}} \mathrm{CNC}\right) \mathrm{Fe}\left(\mathrm{PMe}_{3}\right)\left(\mathrm{N}_{2}\right)$ from $\left({ }^{\mathrm{BPin}} \mathrm{CNC}\right) \mathrm{Fe}\left(\mathrm{PMe}_{3}\right)_{2}\left(6-\mathrm{PMe}_{3}\right)$. Spectra are provided below. The $\mathrm{PMe}_{3} \rightarrow \mathrm{Ph}\left(\mathrm{CH}_{2}\right)_{2} \mathrm{BBN}$ adduct is challenging to observe due to the highly dilute conditions and potential equilibrium processes.

Reaction between ( $\left.{ }^{\mathrm{BPin}} \mathrm{CNC}\right) \mathrm{Fe}\left(\mathrm{PMe}_{3}\right)_{2}$ (6-PMe $)_{3}$ ) and 9-BBN (2 equiv.) in cyclohexane. $\mathrm{A} 20 \mathrm{~mL}$ scintillation vial was charged with ( $\left.{ }^{\mathrm{BPin}} \mathrm{CNC}\right) \mathrm{Fe}\left(\mathrm{PMe}_{3}\right)_{2}\left(6-\mathrm{PMe}_{3}\right)\left(1.000 \mathrm{~mL}, 1.033 \times 10^{-3} \mathrm{M}\right.$ stock solution in cyclohexane, $0.001 \mathrm{mmol})$ and 9-BBN $\left(0.043 \mathrm{~mL}, 4.835 \times 10^{-2} \mathrm{M}\right.$ stock solution in cyclohexane, $\left.0.002 \mathrm{mmol}\right)$. The solution was transferred to a J-Young NMR tube and analysis by NMR spectroscopy was performed after 40 min of mixing. Authentic NMR spectra of both starting materials (stock solution concentration) were also obtained for comparison. The spectral analysis reveals no change to the $\left({ }^{\mathrm{BPin}} \mathrm{CNC}\right) \mathrm{Fe}\left(\mathrm{PMe}_{3}\right)_{2}\left(6-\mathrm{PMe}_{3}\right)$ starting material after $40 \mathrm{~min}$. Subsequent NMR spectra obtained after $48 \mathrm{hr}$ and $96 \mathrm{hr}$ show the appearance of a new species, $\left({ }^{\mathrm{BPin}} \mathrm{CNC}\right) \mathrm{Fe}\left(\mathrm{PMe}_{3}\right)\left(\mathrm{N}_{2}\right)$. The solution was dried, mixed with dry $\mathrm{KBr}$, and pressed into a $\mathrm{KBr}$ pellet. Analysis by infrared spectroscopy is consistent with the presence of a dinitrogen ligand $\left(1974 \mathrm{~cm}^{-1}\right)$. This matches the stretching frequency observed in the material from the reaction with $\mathrm{PhCH}_{2} \mathrm{CH}_{2} \mathrm{BBN}$ (1975 $\mathrm{cm}^{-1} ; \mathrm{KBr}$; spectrum provided below).

Protonation of $\left({ }^{\mathrm{BPin}} \mathrm{CNC}\right) \mathrm{Fe}\left(\mathrm{PMe}_{3}\right)_{2}\left(6-\mathrm{PMe}_{3}\right)$ to generate $\left[\right.$ trans $\left.-\left({ }^{\mathrm{BPin}} \mathrm{CNC}\right) \mathrm{Fe}\left(\mathrm{PMe}_{3}\right)_{2} \mathrm{H}\right]\left[\mathrm{BAr}{ }_{24}\right] . \mathrm{A} 20 \mathrm{~mL}$ scintillation vial was charged with $\left({ }^{\mathrm{BPin}} \mathrm{CNC}\right) \mathrm{Fe}\left(\mathrm{PMe}_{3}\right)_{2}\left(6-\mathrm{PMe}_{3}\right)\left(0.600 \mathrm{~mL}, 6.8844 \times 10^{-3} \mathrm{M}\right.$ stock solution

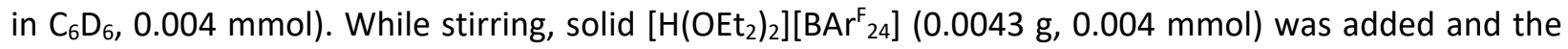
solution transferred to a J-Young NMR tube and sealed. After $10 \mathrm{~min}$, NMR spectra were recorded. Analysis revealed a mixture of cis- and $\left[\right.$ trans- $\left.\left({ }^{\mathrm{BPin}} \mathrm{CNC}\right) \mathrm{Fe}\left(\mathrm{PMe}_{3}\right)_{2}(\mathrm{H})\right]\left[\mathrm{BAr}{ }_{24}\right]$ (1:2 ratio). Select NMR data for $\left[\mathrm{cis}-\left({ }^{\mathrm{BPin}} \mathrm{CNC}\right) \mathrm{Fe}\left(\mathrm{PMe}_{3}\right)_{2}(\mathrm{H})\right]\left[\mathrm{BAr}{ }_{24}\right]:{ }^{1} \mathrm{H} \mathrm{NMR}\left(\mathrm{C}_{6} \mathrm{D}_{6}, 25{ }^{\circ} \mathrm{C}, 700 \mathrm{MHz}\right) \delta=-12.90\left(\mathrm{dd},{ }^{2} J_{\mathrm{P}, \mathrm{H}}=113.7,18.9\right.$, Fe- $H$ ). ${ }^{31} \mathrm{P}$ NMR $\left(\mathrm{C}_{6} \mathrm{D}_{6}, 25{ }^{\circ} \mathrm{C}, 283 \mathrm{MHz}\right) \delta=10.76\left(\mathrm{dd},{ }^{2} J_{\mathrm{P}, \mathrm{P}}=37.7\right), 14.05$ (dd, $\left.{ }^{2} J_{\mathrm{P}, \mathrm{P}}=39.1\right)$. The J-Young tube was stored at room temperature in the dark for $16 \mathrm{hr}$, then reanalyzed by NMR spectroscopy. Analysis revealed primarily $\left[\right.$ trans- $\left.\left({ }^{\mathrm{BPin}} \mathrm{CNC}\right) \mathrm{Fe}\left(\mathrm{PMe}_{3}\right)_{2}(\mathrm{H})\right]\left[\mathrm{BAr}{ }_{24}{ }_{24}{ }^{1} \mathrm{H}\right.$ NMR $\left(\mathrm{C}_{6} \mathrm{D}_{6}, 25{ }^{\circ} \mathrm{C}, 700 \mathrm{MHz}\right) \delta=-12.98\left(\mathrm{t},{ }^{2} J_{\mathrm{P}, \mathrm{H}}\right.$ $=72.1,1 \mathrm{H}, \mathrm{Fe}-\mathrm{H}), 0.16\left(\mathrm{t}, J=3.4,18 \mathrm{H}, \mathrm{P}\left(\mathrm{CH}_{3}\right)_{3}\right), 0.92\left(\mathrm{t}, J=7.6,2 \mathrm{H}, \mathrm{B}-\mathrm{CH}_{2}\right), 1.12\left(\mathrm{~s}, 24 \mathrm{H}, \mathrm{BPin}-\mathrm{CH}_{3}\right), 1.79$ (p, $\left.J=8.5,2 \mathrm{H}, \mathrm{CH}_{2} \mathrm{CH}_{2} \mathrm{CH}_{2}\right), 3.89\left(\mathrm{t}, J=8.7,2 \mathrm{H}, \mathrm{N}-\mathrm{CH}_{2}\right), 6.38$ (d, J = 2.2, 2H, IM-CH), 6.50 (d, J = 7.9, 2H, m-pyr$\mathrm{CH}), 6.88(\mathrm{~d}, J=2.3,2 \mathrm{H}, \mathrm{IM}-\mathrm{CH}), 6.98(\mathrm{t}, J=7.9,1 \mathrm{H}, p$-pyr- $\mathrm{CH}), 7.64(\mathrm{~s}, 4 \mathrm{H}, p-\mathrm{Ar}-\mathrm{CH}), 8.42(\mathrm{~s}, 8 \mathrm{H}, o-\mathrm{Ar}-\mathrm{CH})$. ${ }^{31} \mathrm{P} N M R\left(\mathrm{C}_{6} \mathrm{D}_{6}, 25^{\circ} \mathrm{C}, 283 \mathrm{MHz}\right) \delta=24.85\left(\mathrm{~d},{ }^{2} \mathrm{~J}_{\mathrm{P}, \mathrm{H}}=72.1, P\left(\mathrm{CH}_{3}\right)_{3}\right) .{ }^{11} \mathrm{~B}$ NMR $\left(\mathrm{C}_{6} \mathrm{D}_{6}, 25^{\circ} \mathrm{C}, 283 \mathrm{MHz}\right) \delta=-5.89$ $\left(B A r_{4}\right), 33.74\left(B_{2} \mathrm{C}_{2} \mathrm{Me}_{4}\right)$. MALDI-TOF for [M]-H $\left(\mathrm{C}_{35} \mathrm{H}_{62} \mathrm{~N}_{5} \mathrm{O}_{2} \mathrm{~B}_{2} \mathrm{P}_{2} \mathrm{Fe}_{1}-\mathrm{H}\right)$ : Calc. 755.3734; Found 755.499. 


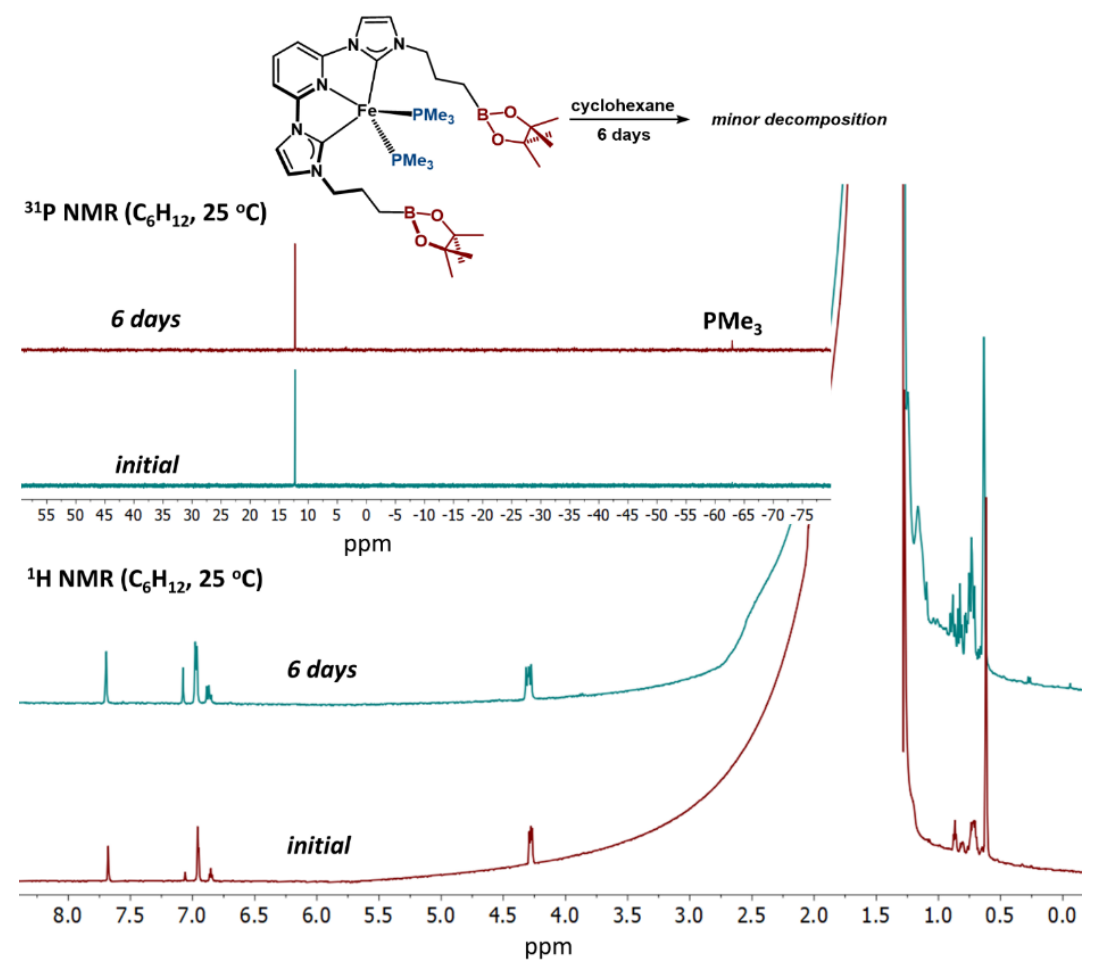

Figure S49 ${ }^{1} \mathrm{H}$ NMR spectra (bottom, $\mathrm{C}_{6} \mathrm{H}_{12}, 25{ }^{\circ} \mathrm{C}, 400 \mathrm{MHz}$ ) and ${ }^{31} \mathrm{P} \mathrm{NMR}$ spectra (top, $162 \mathrm{MHz}$ ) corresponding to $\left({ }^{\mathrm{BPin}} \mathrm{CNC}\right) \mathrm{Fe}\left(\mathrm{PMe}_{3}\right)_{2}\left(6-\mathrm{PMe}_{3}\right)$ at an initial time point and after 6 days. Minimal degradation is observed. 


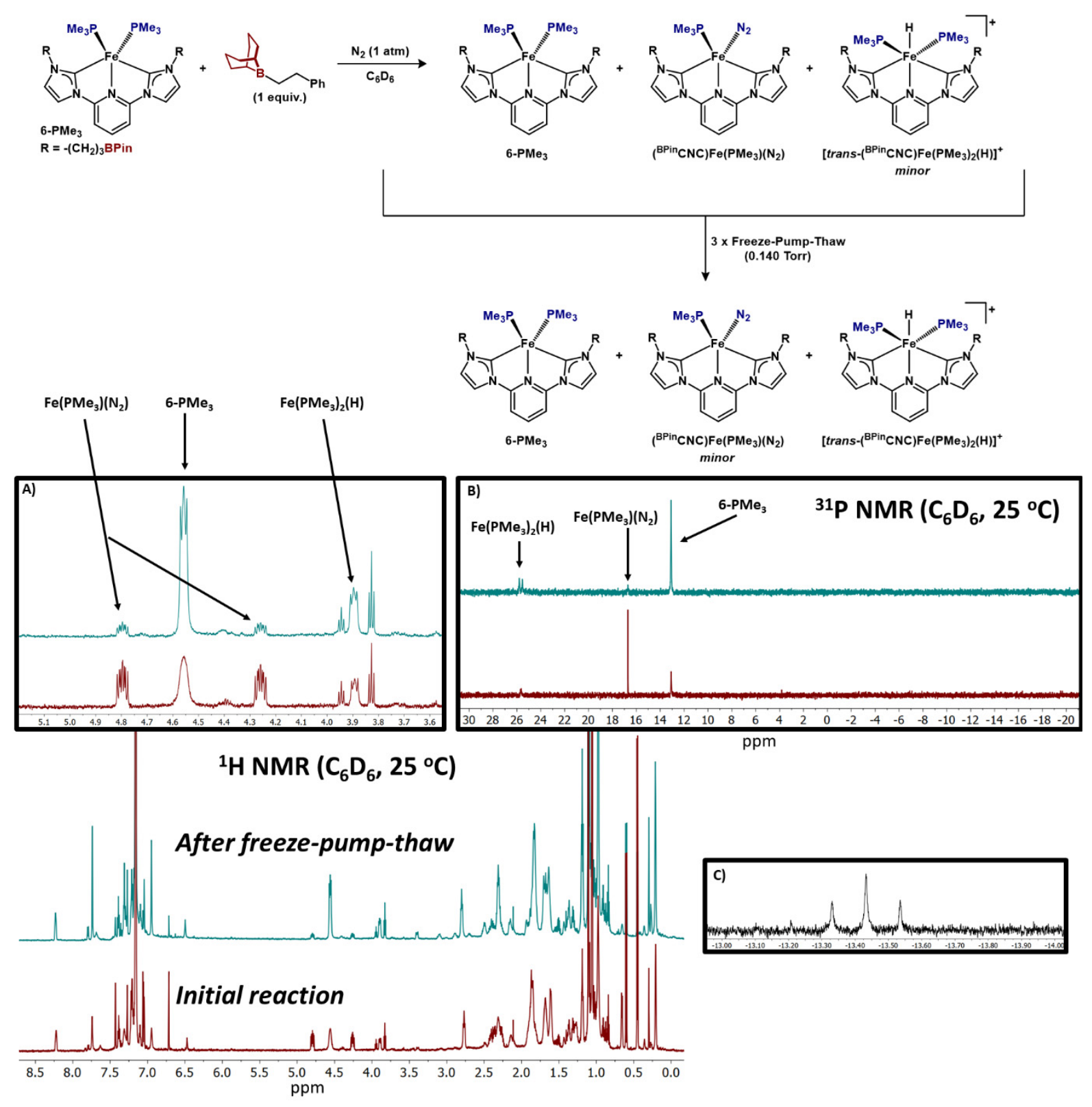

Figure $\mathrm{S} 50 \mathrm{NMR}$ spectra $\left(\mathrm{C}_{6} \mathrm{D}_{6}, 25^{\circ} \mathrm{C}, 400 \mathrm{MHz}\right)$ corresponding to the reaction scheme at the top of the figure. The bottom left displays the initial reaction stacked with the result of subjecting the J-Young tube to $3 \times$ freeze-pump-thaw cycles (0.140 Torr). Inset A displays diagnostic ${ }^{1} \mathrm{H}$ NMR resonances for each of the three species observed in solution. Inset $B$ is the corresponding ${ }^{31} \mathrm{P}$ NMR spectra (162 MHz). Inset $C$ displays the diagnostic hydride resonance for $\left[\left({ }^{\mathrm{BPin}} \mathrm{CNC}\right) \mathrm{Fe}\left(\mathrm{PMe}_{3}\right)_{2}(\mathrm{H})\right]^{+} \cdot{ }^{14}$ 

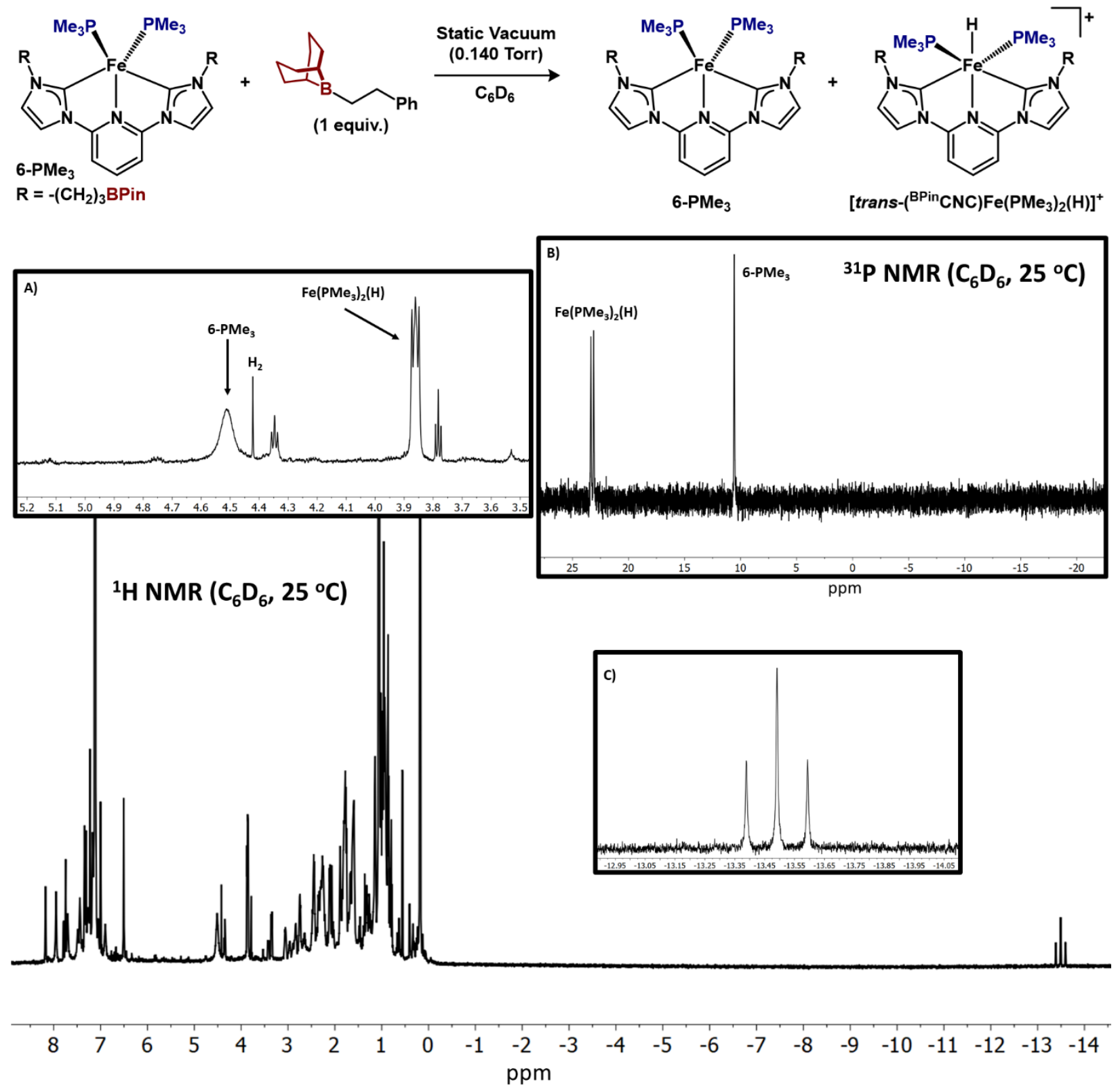

Figure S51 NMR spectra $\left(\mathrm{C}_{6} \mathrm{D}_{6}, 25^{\circ} \mathrm{C}, 700 \mathrm{MHz}\right)$ corresponding to the reaction scheme at the top of the figure. The bottom left displays the full ${ }^{1} \mathrm{H}$ NMR spectrum. Inset A displays diagnostic ${ }^{1} \mathrm{H}$ NMR resonances for each of the two species iron-containing species observed in solution. Inset $B$ is the corresponding ${ }^{31} \mathrm{P}$ NMR spectra (283 MHz). Inset $C$ displays the diagnostic hydride resonance for $\left[\left({ }^{\mathrm{BPin}} \mathrm{CNC}\right) \mathrm{Fe}\left(\mathrm{PMe}_{3}\right)_{2}(\mathrm{H})\right]^{+} .^{13}$ 


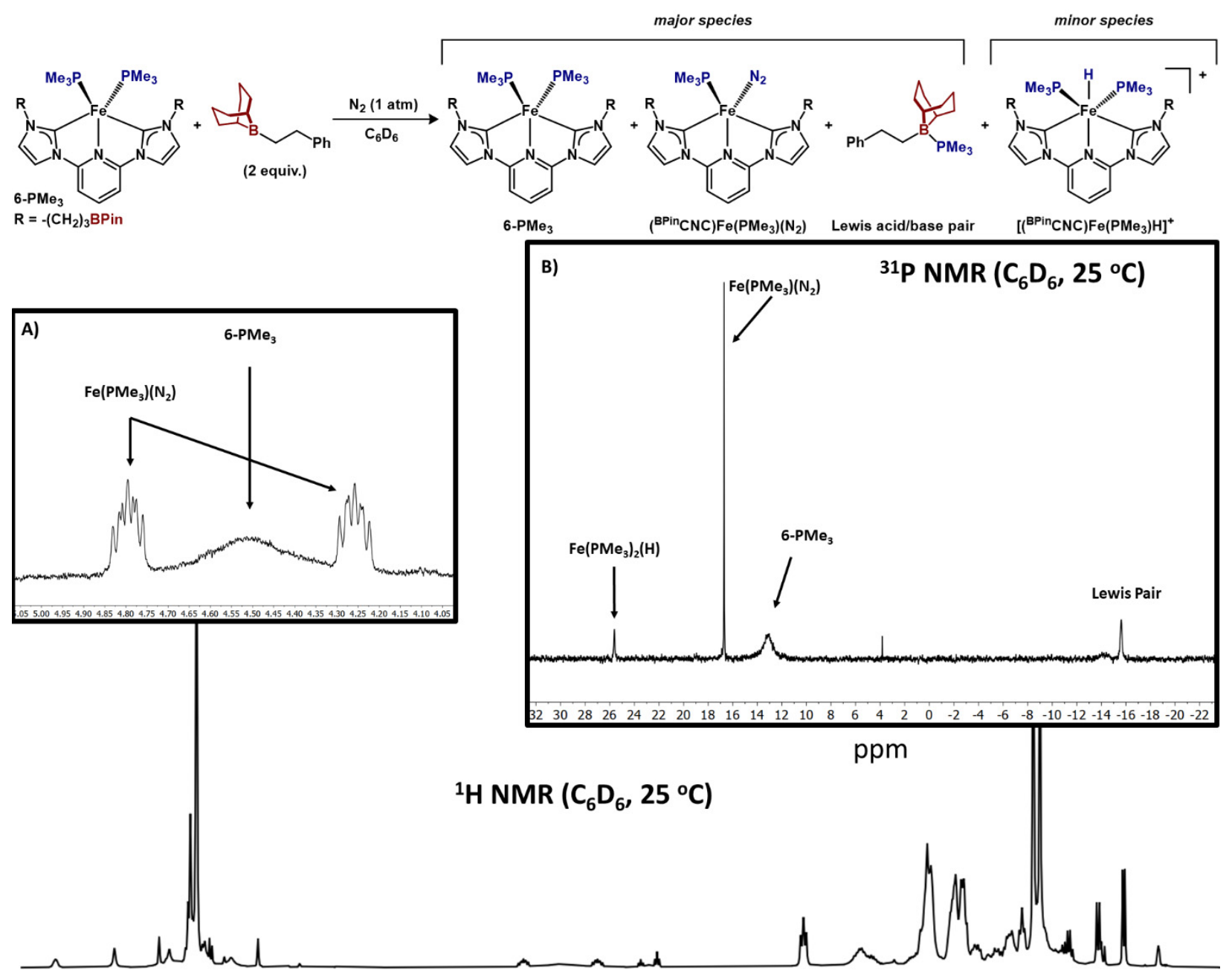

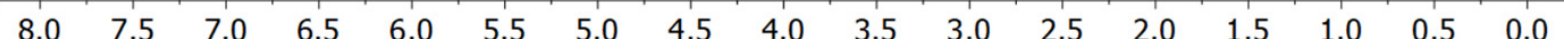

Figure S52 NMR spectra $\left(\mathrm{C}_{6} \mathrm{D}_{6}, 25^{\circ} \mathrm{C}, 700 \mathrm{MHz}\right)$ corresponding to the reaction scheme at the top of the figure. The bottom displays the full ${ }^{1} \mathrm{H}$ NMR spectrum. Inset A displays diagnostic ${ }^{1} \mathrm{H}$ NMR resonances for each of the two major species iron-containing species observed in solution. Inset $B$ is the corresponding ${ }^{31} \mathrm{P}$ NMR spectra $(283 \mathrm{MHz})$. 

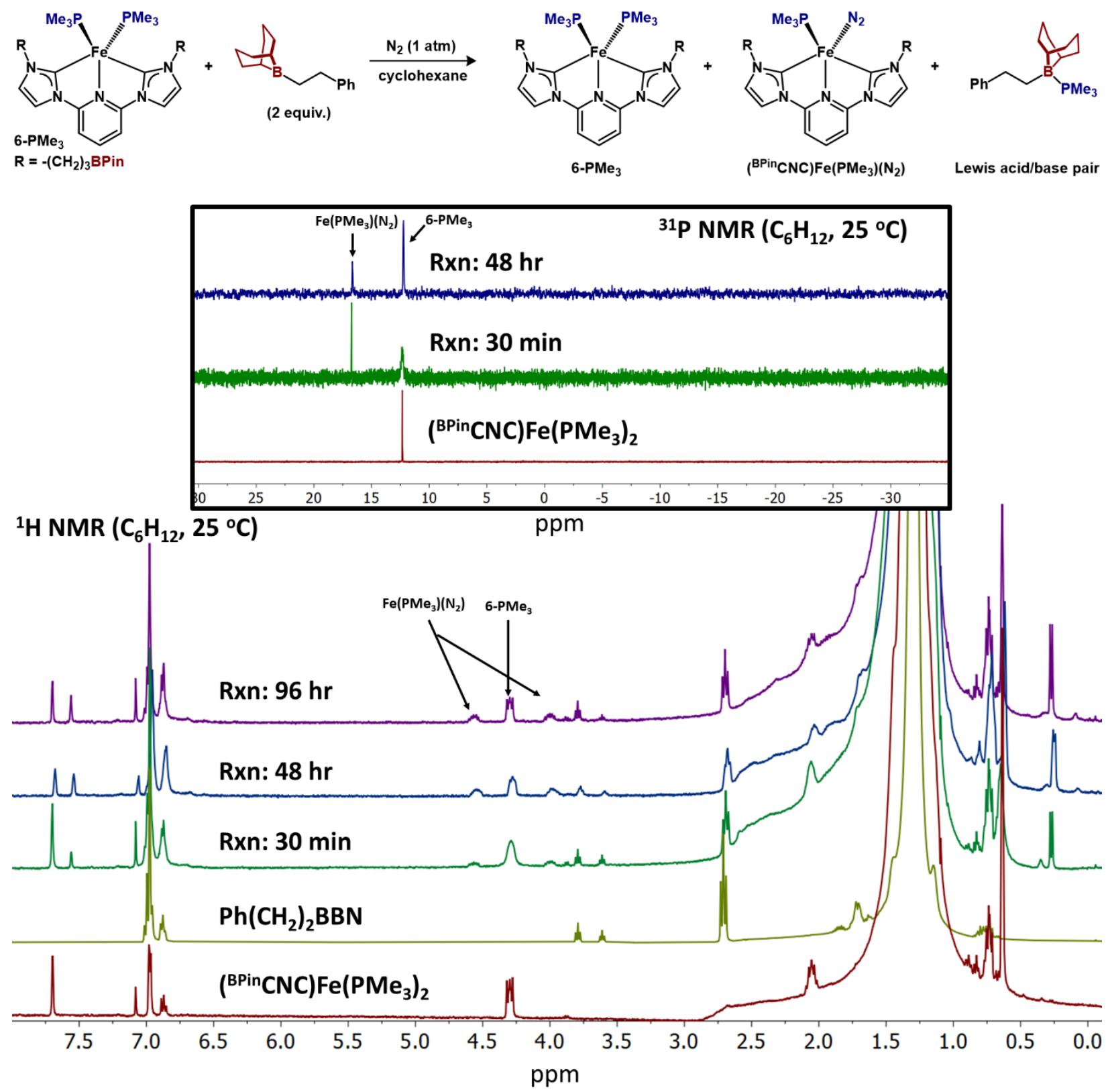

Figure S53 NMR spectra $\left(\mathrm{C}_{6} \mathrm{H}_{12}, 25^{\circ} \mathrm{C}, 400 \mathrm{MHz}\right)$ corresponding to the reaction scheme at the top of the figure. The bottom displays the full ${ }^{1} \mathrm{H}$ NMR spectrum of the two starting materials and three different time points of the reaction. The inset is the corresponding ${ }^{31} \mathrm{P}$ NMR spectra (162 MHz). 

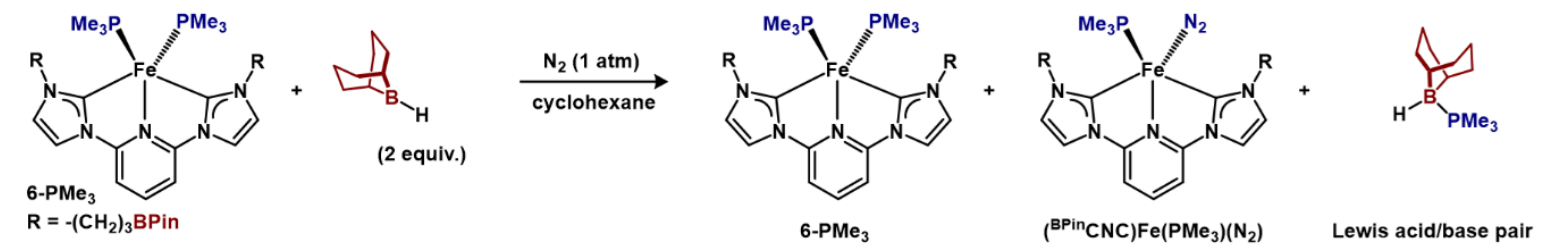

${ }^{31}$ P NMR $\left(\mathrm{C}_{6} \mathrm{H}_{12}, 25^{\circ} \mathrm{C}\right)$
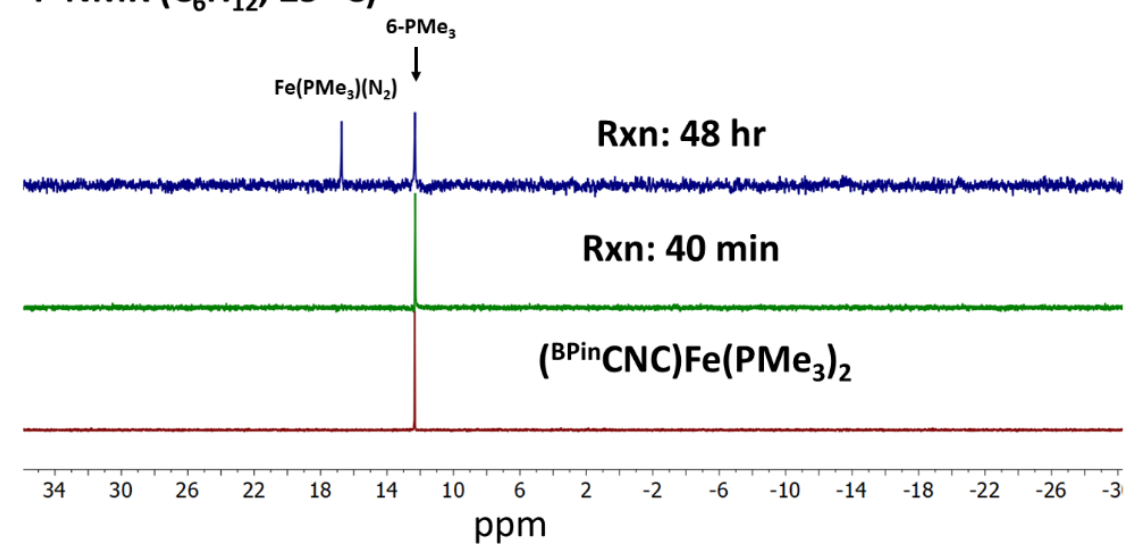

\section{Rxn: 40 min}

${ }^{1} \mathrm{H}$ NMR $\left(\mathrm{C}_{6} \mathrm{H}_{12}, 25^{\circ} \mathrm{C}\right)$

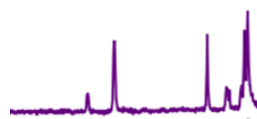

Rxn: $96 \mathrm{hr}$
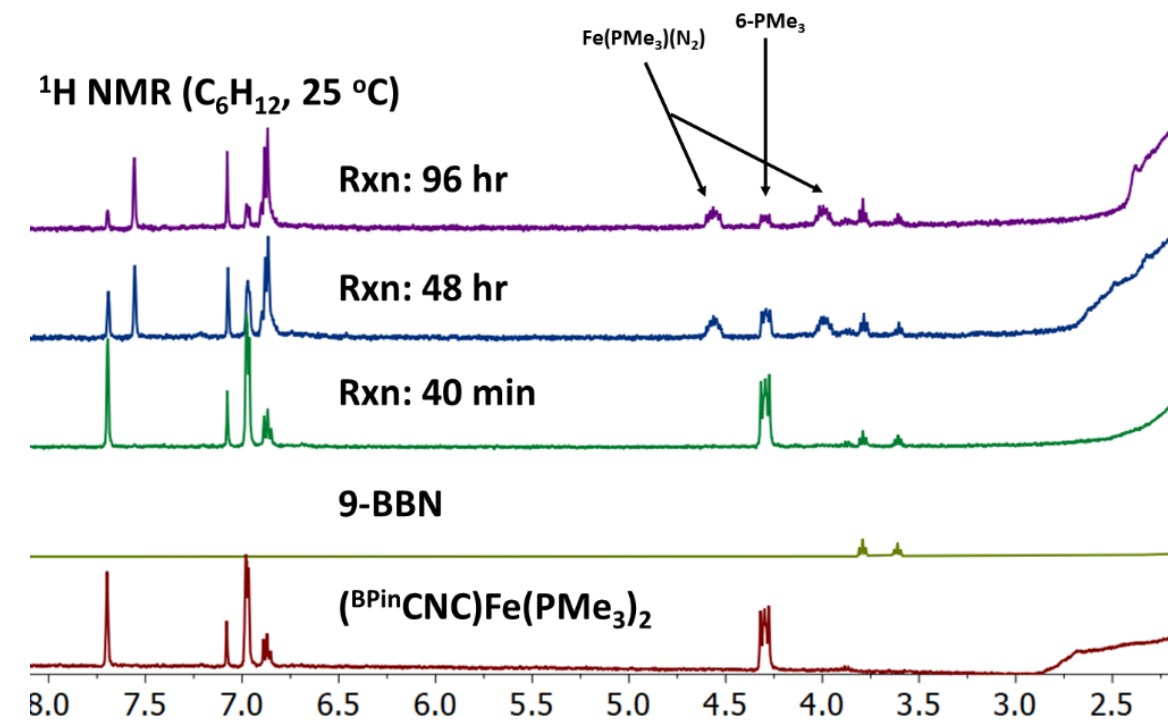

$$
\text { ppm }
$$

Figure S54 ${ }^{1} \mathrm{H}$ NMR spectra (bottom, cyclohexane, $25^{\circ} \mathrm{C}, 400 \mathrm{MHz}$ ) and ${ }^{31} \mathrm{P}$ NMR spectra (top, $162 \mathrm{MHz}$ ) corresponding to addition of $9-\mathrm{BBN}$ to $\left({ }^{\mathrm{BPin}} \mathrm{CNC}\right) \mathrm{Fe}\left(\mathrm{PMe}_{3}\right)_{2}\left(6-\mathrm{PMe}_{3}\right)$. The reaction was performed in cyclohexane. 

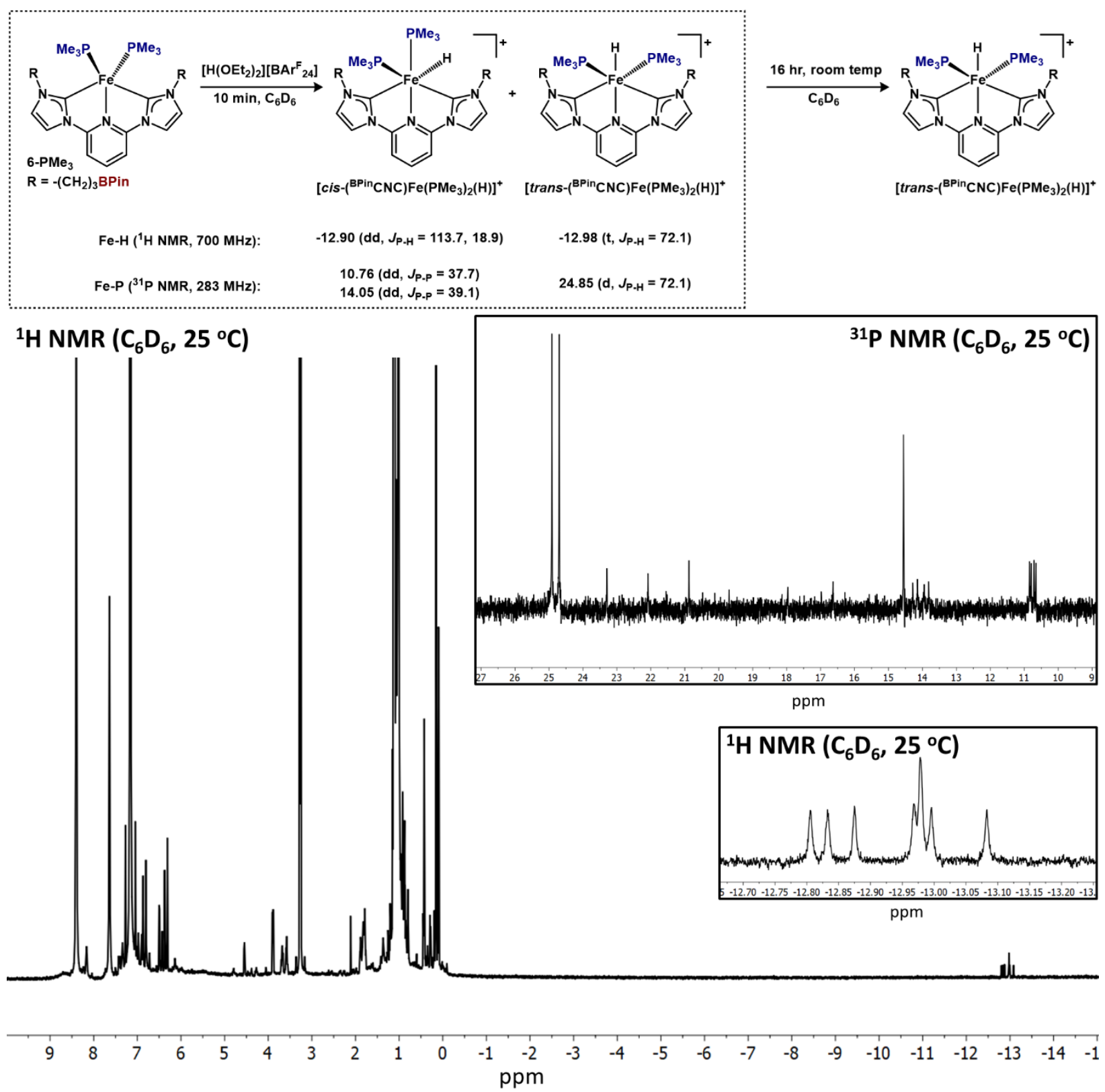

Figure S55 ${ }^{1} \mathrm{H}$ NMR spectrum $\left(\mathrm{C}_{6} \mathrm{D}_{6}, 25{ }^{\circ} \mathrm{C}, 700 \mathrm{MHz}\right)$ and ${ }^{31} \mathrm{P}$ NMR spectrum (inset, $283 \mathrm{MHz}$ ) corresponding to a mixture of cis- and $\left[\right.$ trans- $\left.\left({ }^{\mathrm{BPin}} \mathrm{CNC}\right) \mathrm{Fe}\left(\mathrm{PMe}_{3}\right)_{2}(\mathrm{H})\right]\left[\mathrm{BAr}{ }_{24}\right]$ generated by the addition of $\left[\mathrm{H}\left(\mathrm{OEt}_{2}\right)_{2}\right]\left[\mathrm{BAr}_{24}{ }_{24}\right]$ to 6-PMe $\mathrm{PM}_{3}$. The reaction is depicted at the top of the figure. These crude spectra were recorded ca. $10 \mathrm{~min}$ after addition of the acid. 


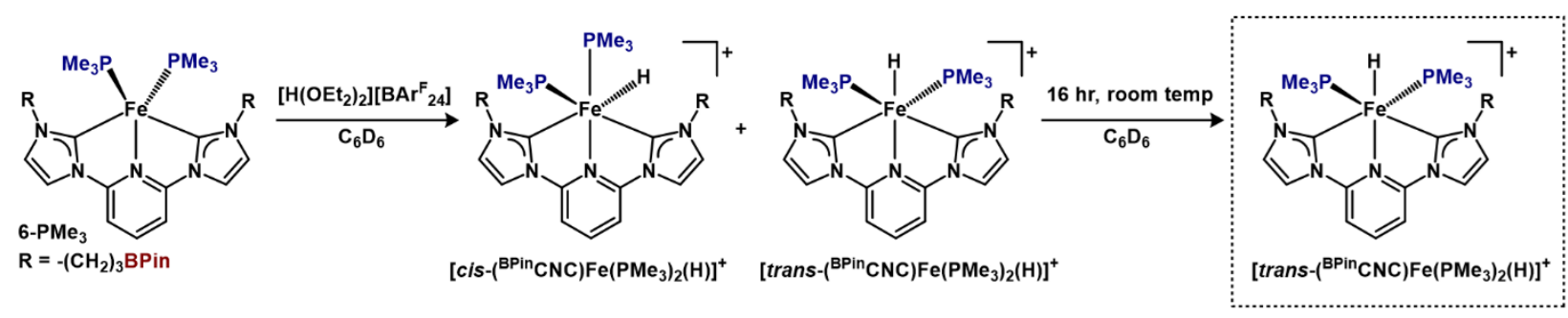

$\mathrm{Fe}-\mathrm{H}\left({ }^{1} \mathrm{H}\right.$ NMR, $\left.700 \mathrm{MHz}\right): \quad-12.90\left(\mathrm{dd}, J_{\mathrm{P}-\mathrm{H}}=113.7,18.9\right) \quad-12.98\left(\mathrm{t}, J_{\mathrm{P}-\mathrm{H}}=72.1\right)$

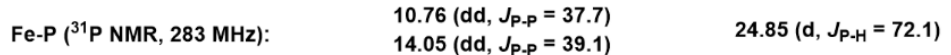

${ }^{1} \mathrm{H}$ NMR $\left(C_{6} D_{6}, 25^{\circ} \mathrm{C}\right)$

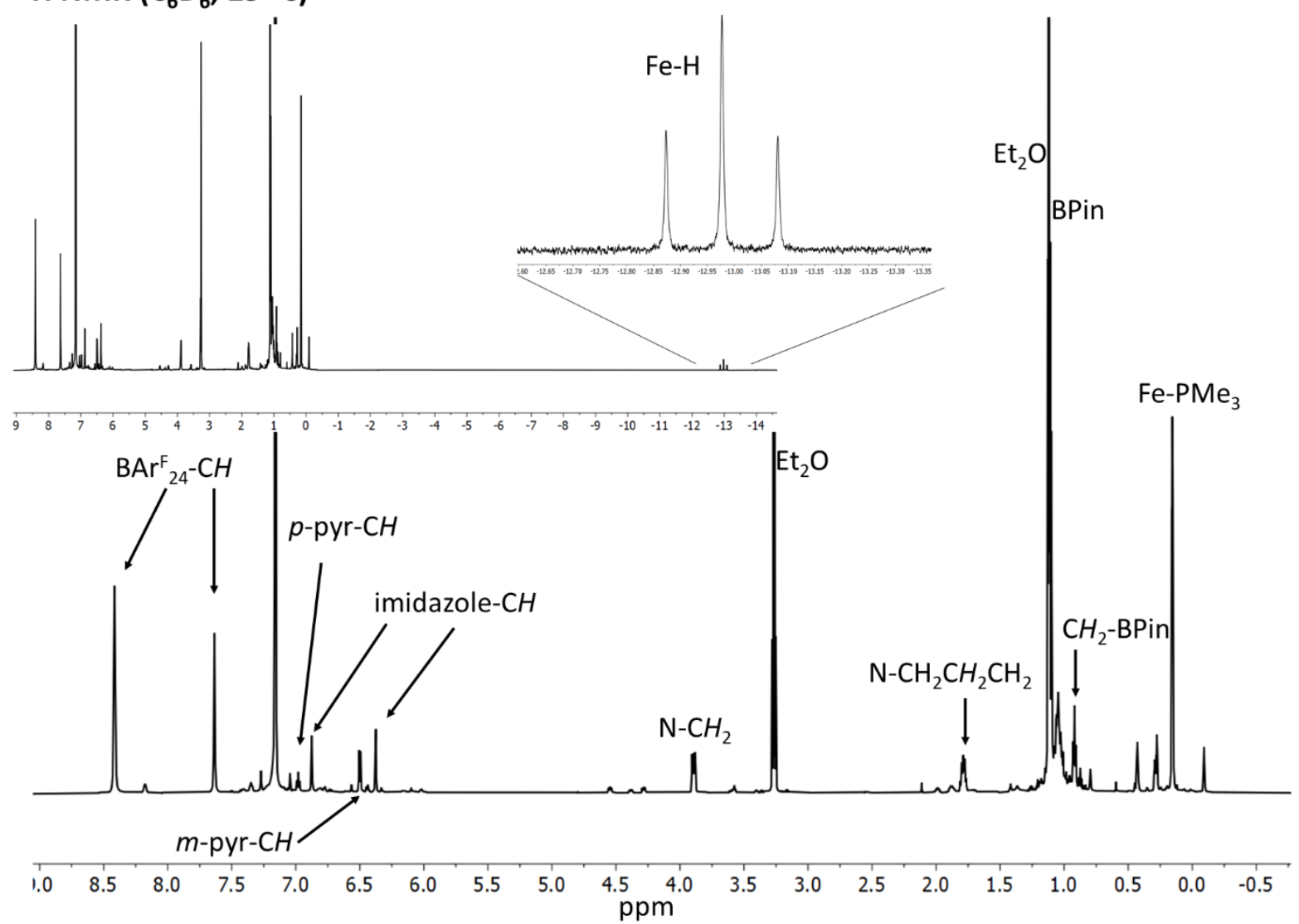

Figure S56 ${ }^{1} \mathrm{H} \quad \mathrm{NMR}$ spectrum $\left(\mathrm{C}_{6} \mathrm{D}_{6}, 25{ }^{\circ} \mathrm{C}, \quad 700 \mathrm{MHz}\right)$ corresponding to [trans$\left.\left({ }^{\mathrm{BPin}} \mathrm{CNC}\right) \mathrm{Fe}\left(\mathrm{PMe}_{3}\right)_{2}(\mathrm{H})\right]\left[\mathrm{BAr}{ }_{24}\right]$ generated by the addition of $\left[\mathrm{H}\left(\mathrm{OEt}_{2}\right)_{2}\right]\left[\mathrm{BAr}^{\mathrm{F}}{ }_{24}\right]$ to 6-PMe $\mathbf{P}_{3}$. The reaction is depicted at the top of the figure. This crude spectrum was recorded $16 \mathrm{hr}$ after addition of the acid. 

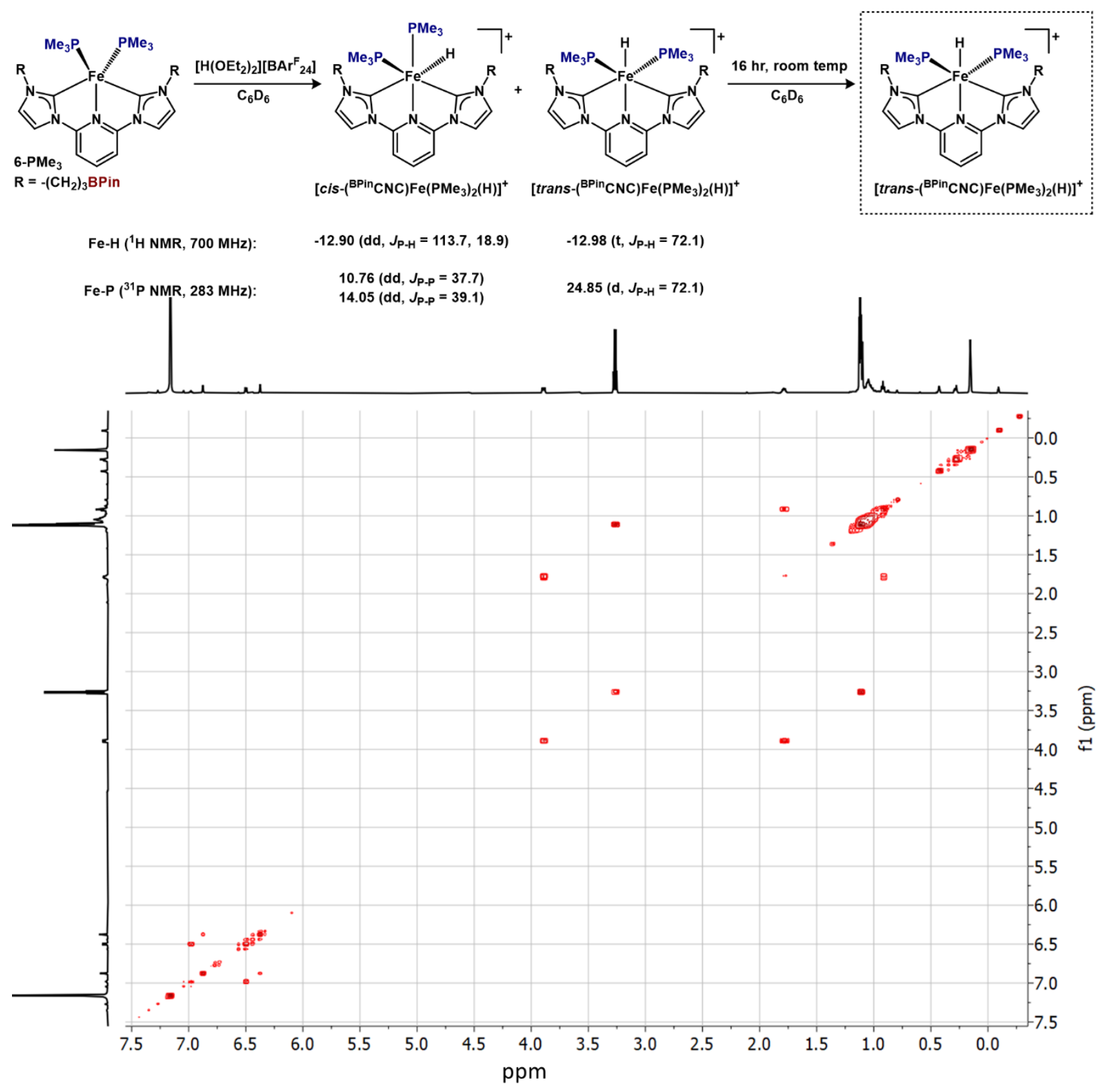

Figure S57 $\quad{ }^{1} \mathrm{H}-{ }^{1} \mathrm{H} \quad$ COSY spectrum $\left(\mathrm{C}_{6} \mathrm{D}_{6}, \quad 25 \quad{ }^{\circ} \mathrm{C}, \quad 700 \mathrm{MHz}\right)$ corresponding to [trans$\left.\left({ }^{\mathrm{BPin}} \mathrm{CNC}\right) \mathrm{Fe}\left(\mathrm{PMe}_{3}\right)_{2}(\mathrm{H})\right]\left[\mathrm{BAr}{ }_{24}\right]$ generated by the addition of $\left[\mathrm{H}\left(\mathrm{OEt}_{2}\right)_{2}\right]\left[\mathrm{BAr}^{\mathrm{F}}{ }_{24}\right]$ to 6-PMe $\mathbf{P}_{3}$. The reaction is depicted at the top of the figure. This crude spectrum was recorded $16 \mathrm{hr}$ after addition of the acid. 

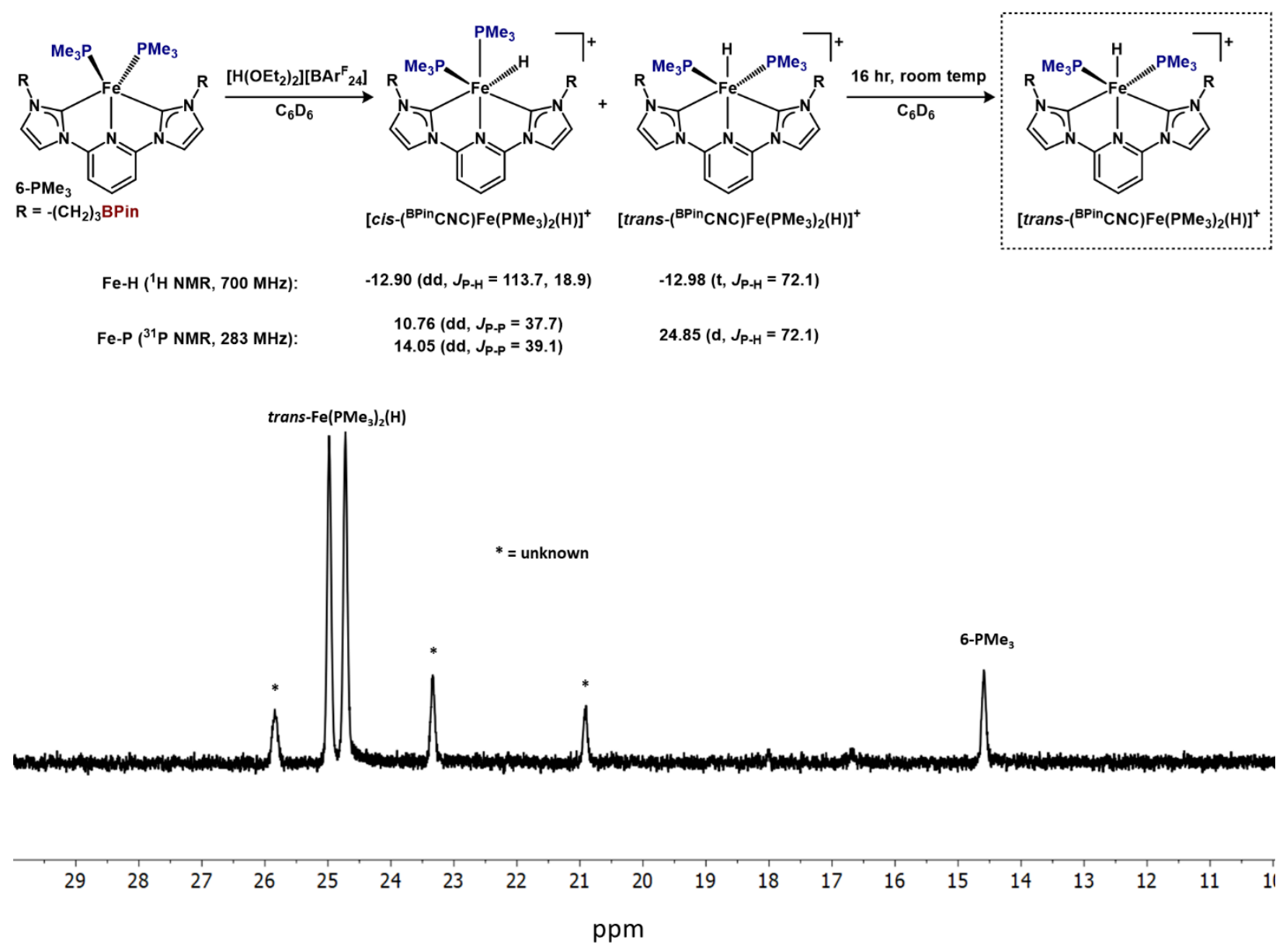

Figure S58 ${ }^{31} \mathrm{P} \quad \mathrm{NMR}$ spectrum $\left(\mathrm{C}_{6} \mathrm{D}_{6}, 25{ }^{\circ} \mathrm{C}, \quad 700 \mathrm{MHz}\right)$ corresponding to [trans$\left.\left({ }^{\mathrm{BPin}} \mathrm{CNC}\right) \mathrm{Fe}\left(\mathrm{PMe}_{3}\right)_{2}(\mathrm{H})\right]\left[\mathrm{BAr}_{24}{ }_{24}\right]$ generated by the addition of $\left[\mathrm{H}\left(\mathrm{OEt}_{2}\right)_{2}\right]\left[\mathrm{BAr}^{\mathrm{F}}{ }_{24}\right]$ to 6-PMe $\mathbf{P}_{3}$. The reaction is depicted at the top of the figure. This crude spectrum was recorded $16 \mathrm{hr}$ after addition of the acid. 


\section{Ligand Substitution Reactions}

\section{Formation of ( $\left.{ }^{\mathrm{BPin}} \mathrm{CNC}\right) \mathrm{Fe}(\mathrm{CO})_{2}$ from $\left({ }^{\mathrm{BPin}} \mathrm{CNC}\right) \mathrm{Fe}\left(\mathrm{PMe}_{3}\right)_{2}\left(6-\mathrm{PMe}_{3}\right)$.}

A) Reaction in benzene- $d_{6}\left(\mathrm{C}_{6} \mathrm{D}_{6}\right)$ : A J-Young NMR tube was charged with ( $\left.{ }^{\mathrm{BPin}} \mathrm{CNC}\right) \mathrm{Fe}\left(\mathrm{PMe}_{3}\right)_{2}\left(6-\mathrm{PMe}_{3}\right)$ $(0.001 \mathrm{~g}, 0.001 \mathrm{mmol})$ and $0.6 \mathrm{~mL} \mathrm{C}_{6} \mathrm{D}_{6}$. Initial ${ }^{1} \mathrm{H}$ and ${ }^{31} \mathrm{P}$ NMR spectra were obtained. The tube was charged with $20 \mathrm{psig} \mathrm{CO}$. After $10 \mathrm{~min},{ }^{1} \mathrm{H}$ and ${ }^{31} \mathrm{P}$ NMR spectra were acquired and revealed complete conversion to $\left({ }^{\mathrm{BPin}} \mathrm{CNC}\right) \mathrm{Fe}(\mathrm{CO})_{2}$ and displacement of $\mathrm{PMe}_{3}{ }^{1}$ Three freeze-pump-thaw cycles were performed on the J-Young tube and the solution left for $24 \mathrm{hr} .{ }^{1} \mathrm{H}$ and ${ }^{31} \mathrm{P} \mathrm{NMR}$ spectra were acquired and revealed no change had occurred; only $\left({ }^{\mathrm{BPin}} \mathrm{CNC}\right) \mathrm{Fe}(\mathrm{CO})_{2}$ and uncoordinated $\mathrm{PMe}_{3}$ are observed. Example spectra a provided below.

B) Reaction in cyclohexane $\left(\mathrm{C}_{6} \mathrm{H}_{12}\right)$ : A J-Young NMR tube was charged with $0.600 \mathrm{~mL}$ of $\left({ }^{\mathrm{BPin}} \mathrm{CNC}\right) \mathrm{Fe}(\mathrm{PMe})_{2}$ $\left(6-\mathrm{PMe}_{3}\right)\left(1.033 \times 10^{-3} \mathrm{M}\right.$ stock solution in cyclohexane). Initial ${ }^{1} \mathrm{H}$ and ${ }^{31} \mathrm{P}$ NMR spectra were obtained. The tube was charged with $15 \mathrm{psig} \mathrm{CO}$. After $10 \mathrm{~min},{ }^{1} \mathrm{H}$ and ${ }^{31} \mathrm{P}$ NMR spectra were acquired and revealed complete conversion to $\left({ }^{\mathrm{BPin}} \mathrm{CNC}\right) \mathrm{Fe}(\mathrm{CO})_{2}$ and displacement of $\mathrm{PMe}_{3}{ }^{1}$ Example spectra a provided below.

Sequential addition of $\mathrm{CN}^{t} \mathrm{Bu}$ and $\mathrm{CO}$ to (allyl $\left.\mathrm{CNC}\right) \mathrm{Fe}\left(\mathrm{PMe}_{3}\right)_{2}$ (3-PMe $)_{3}$. A J-Young NMR tube was charged with (allyl $\mathrm{CNC}) \mathrm{Fe}\left(\mathrm{PMe}_{3}\right)_{2}\left(3-\mathrm{PMe}_{3}\right)(0.0057 \mathrm{~g}, 0.011 \mathrm{mmol})$ and $0.600 \mathrm{~mL} \mathrm{C} \mathrm{C}_{6}$. To this solution, tert-butyl isocyanide $\left(0.442 \mathrm{M}\right.$ stock solution in $\mathrm{C}_{6} \mathrm{D}_{6}, 0.052 \mathrm{~mL}, 0.022 \mathrm{mmol}$ ) was added. The tube was sealed. After 7 days, the solution was analyzed by NMR spectroscopy. The data is consistent with formation of $\left({ }^{\text {allyl }} \mathrm{CNC}\right) \mathrm{Fe}\left(\mathrm{PMe}_{3}\right)\left(\mathrm{CN}^{t} \mathrm{Bu}\right)$. One equivalent of each $\mathrm{CN}^{t} \mathrm{Bu}$ and $\mathrm{PMe}_{3}$ is observed. The J-Young NMR tube was then pressurized with 25 psig CO. After 7 days, the solution was analyzed by NMR spectroscopy. The data is consistent with formation of (allyl $\mathrm{CNC}) \mathrm{Fe}(\mathrm{CO})_{2} .{ }^{1}$ Two equivalents of $\mathrm{CN}^{t} \mathrm{Bu}$ and $\mathrm{PMe}_{3}$ are observed. Representative spectra are included below. 


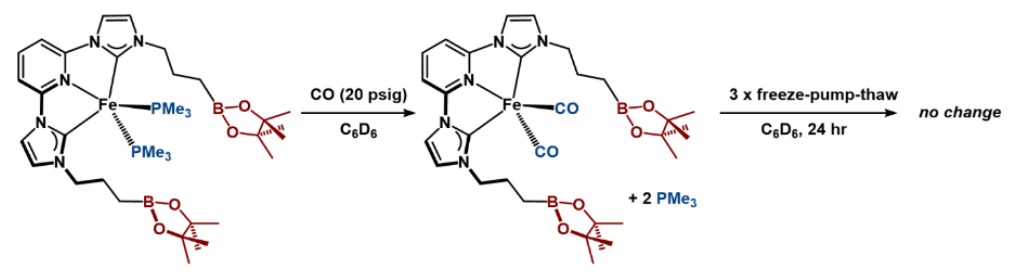

${ }^{31}$ P NMR $\left(C_{6} D_{6}, 25^{\circ} \mathrm{C}\right)$

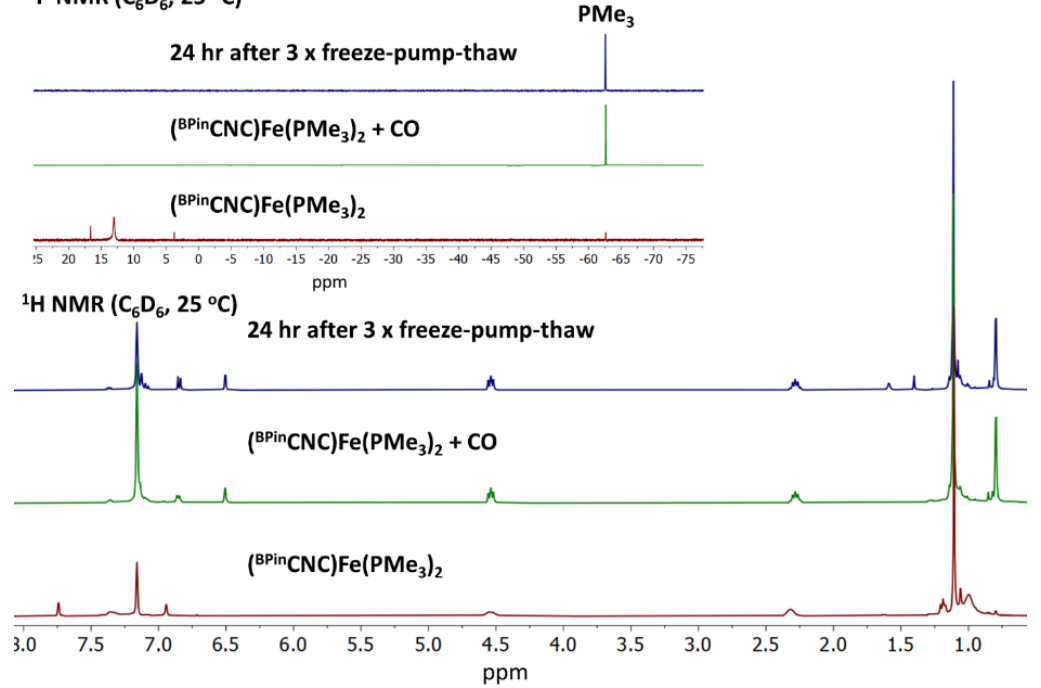

Figure S59 ${ }^{1} \mathrm{H}$ NMR spectra (bottom, $\mathrm{C}_{6} \mathrm{D}_{6}, 25{ }^{\circ} \mathrm{C}, 400 \mathrm{MHz}$ ) and ${ }^{31} \mathrm{P} \mathrm{NMR}$ spectra (top, $162 \mathrm{MHz}$ ) corresponding to addition of $\mathrm{CO}(20 \mathrm{psig})$ to $\left({ }^{\mathrm{BPin}} \mathrm{CNC}\right) \mathrm{Fe}\left(\mathrm{PMe}_{3}\right)_{2}\left(6-\mathrm{PMe}_{3}\right)$.

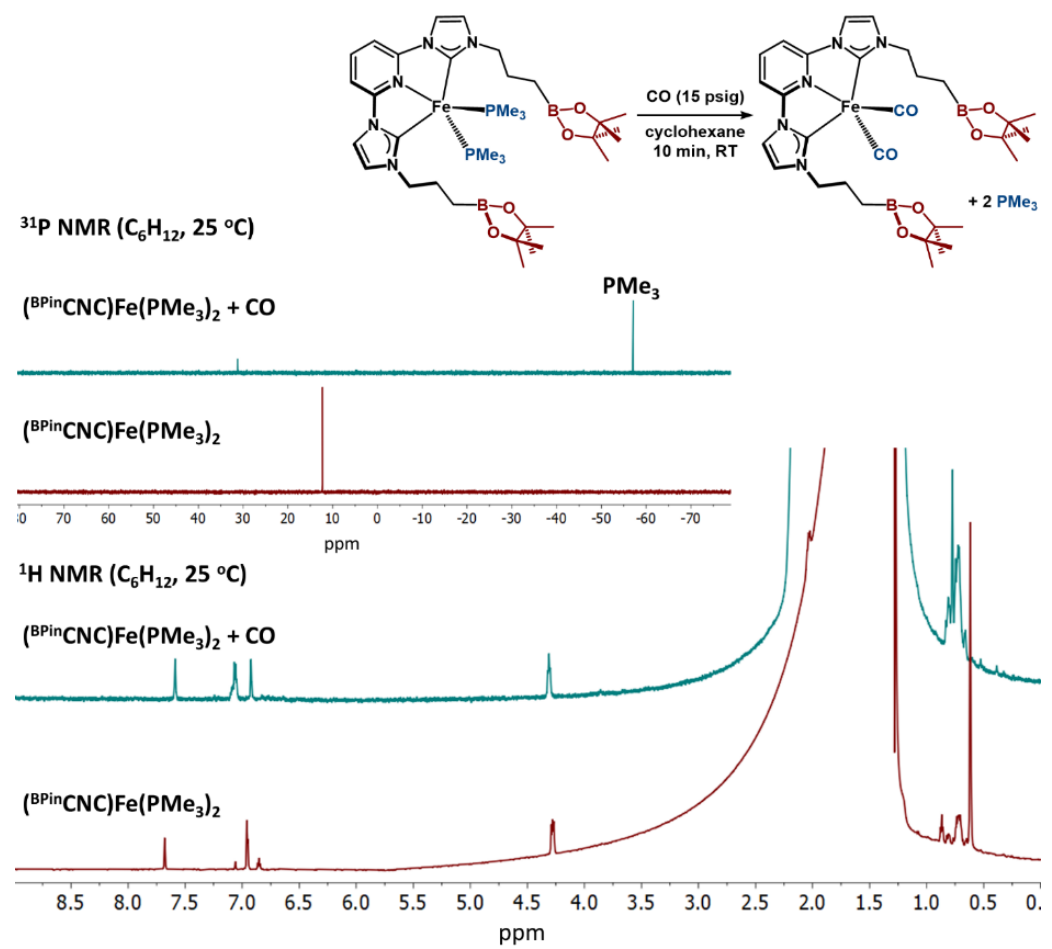

Figure S60 ${ }^{1} \mathrm{H}$ NMR spectra (bottom, $\mathrm{C}_{6} \mathrm{H}_{12}, 25{ }^{\circ} \mathrm{C}, 700 \mathrm{MHz}$ ) and ${ }^{31} \mathrm{P} \mathrm{NMR}$ spectra (top, $283 \mathrm{MHz}$ ) corresponding to addition of $\mathrm{CO}(15 \mathrm{psig})$ to $\left({ }^{\mathrm{BPin}} \mathrm{CNC}\right) \mathrm{Fe}\left(\mathrm{PMe}_{3}\right)_{2}\left(6-\mathrm{PMe}_{3}\right)$. 


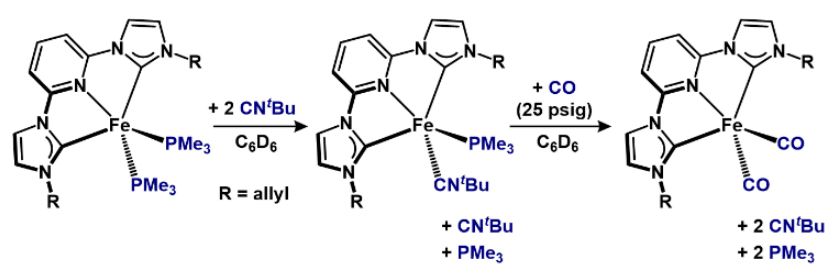

${ }^{1} \mathrm{H}$ NMR $\left(C_{6} D_{6}, 25^{\circ} \mathrm{C}\right)$

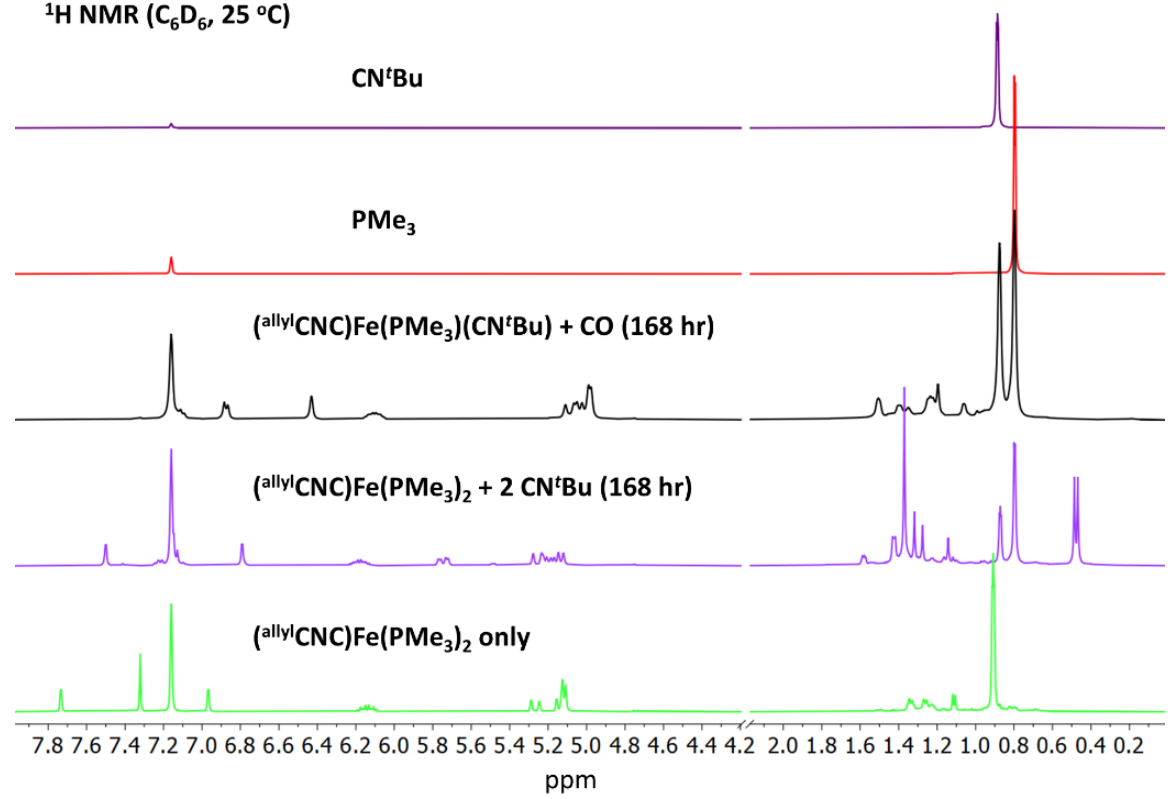

Figure $\mathrm{S} 61{ }^{1} \mathrm{H}$ NMR spectra $\left(\mathrm{C}_{6} \mathrm{D}_{6}, 25^{\circ} \mathrm{C}, 401 \mathrm{MHz}\right)$ of (allyl $\left.\mathrm{CNC}\right) \mathrm{Fe}\left(\mathrm{PMe}_{3}\right)_{2}\left(3-\mathrm{PMe}_{3}\right)$ (bottom), after addition of 2 equiv. $\mathrm{CN}^{t} \mathrm{Bu}$ (purple), and after addition of $\mathrm{CO}$ (black). Authentic spectra of $\mathrm{PMe}_{3}$ (red) and $\mathrm{CN}^{t} \mathrm{Bu}$ (top) are included.

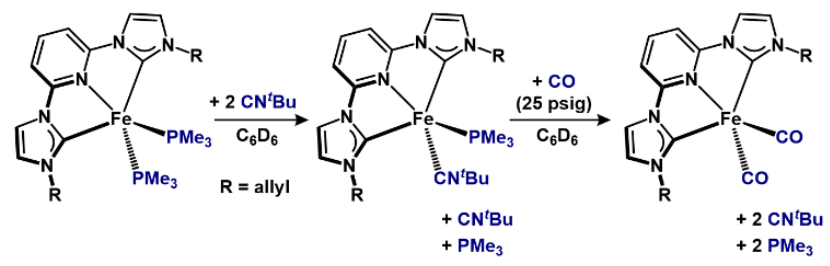

${ }^{31} \mathrm{P}$ NMR $\left(C_{6} D_{6}, 25^{\circ} \mathrm{C}\right)$

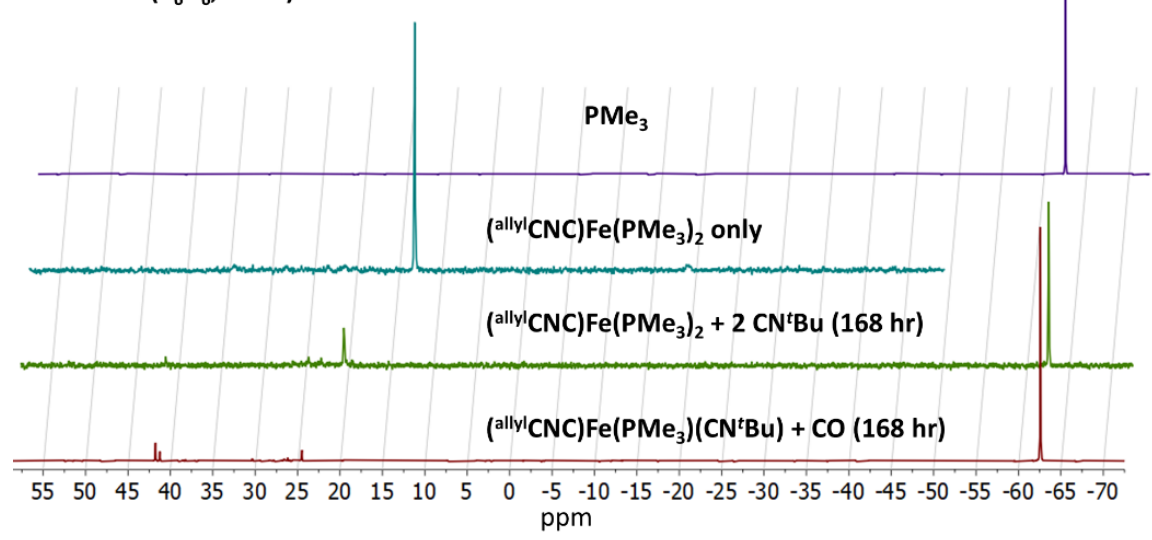

Figure S62 ${ }^{31} \mathrm{P}$ NMR spectra $\left(\mathrm{C}_{6} \mathrm{D}_{6}, 25{ }^{\circ} \mathrm{C}, 162 \mathrm{MHz}\right)$ of (allyl $\left.\mathrm{CNC}\right) \mathrm{Fe}\left(\mathrm{PMe}_{3}\right)_{2}$ (3-PMe $)$, after addition of 2 equiv. $\mathrm{CN}^{t} \mathrm{Bu}$ (green), and after addition of $\mathrm{CO}$ (red). An authentic spectrum of $\mathrm{PMe}_{3}$ (top) is included. 


\section{FLP-Type Chemistry observed with (allyl $\mathrm{CNC}) \mathrm{Fe}(\mathrm{CO})_{2}(1)$.}

Reaction between (allyl $\mathrm{CNC}) \mathrm{Fe}(\mathrm{CO})_{2}(\mathbf{1})$ and $\mathrm{HB}\left(\mathrm{C}_{6} \mathrm{~F}_{5}\right)_{2}$ or $\mathrm{B}\left(\mathrm{C}_{6} \mathrm{~F}_{5}\right)_{3}$ (in situ). A J-Young NMR tube was charged with $1.000 \mathrm{~mL}$ of a stock solution (DME, $8.68 \times 10^{-3} \mathrm{M}, 0.0087 \mathrm{mmol}$ ) of (allyl $\left.\mathrm{CNC}\right) \mathrm{Fe}(\mathrm{CO})_{2}(\mathbf{1})$. To this, $\mathrm{HB}\left(\mathrm{C}_{6} \mathrm{~F}_{5}\right)_{2}(0.159 \mathrm{M}$ stock solution in $\mathrm{DME}, 0.056 \mathrm{~mL}, 0.0088 \mathrm{mmol})$ or $\mathrm{B}\left(\mathrm{C}_{6} \mathrm{~F}_{5}\right)_{3}(0.150 \mathrm{M}$ stock solution in DME, $0.059 \mathrm{~mL}, 0.0089 \mathrm{mmol}$ ) was added and the NMR tube was sealed. After $24 \mathrm{hr}$, the solution was investigated by NMR spectroscopy. The solution was then evaporated to dryness under reduced pressure, mixed with dry $\mathrm{KBr}$, and investigated by infrared spectroscopy. Crude, comparative spectra are provided below.

Reaction between (ally' $\mathrm{CNC}) \mathrm{Fe}(\mathrm{CO})_{2}(1)$ and $\mathrm{B}\left(\mathrm{C}_{6} \mathrm{~F}_{5}\right)_{3}$ to generate cis-(allyl $\left.\mathrm{CNC}\right) \mathrm{Fe}(\mathrm{CO})_{2}\left(\mathrm{C}_{6} \mathrm{~F}_{4}-\mathrm{BF}\left(\mathrm{C}_{6} \mathrm{~F}_{5}\right)_{2}\right)$ (isolation). A $20 \mathrm{~mL}$ scintillation vial was charged with (allyl $\mathrm{CNC}) \mathrm{Fe}(\mathrm{CO})_{2}(\mathbf{1})(0.052 \mathrm{~g}, 0.129 \mathrm{mmol})$ and $8 \mathrm{~mL}$ $\mathrm{C}_{6} \mathrm{H}_{6}$ to form a deep red solution. While stirring, $\mathrm{B}\left(\mathrm{C}_{6} \mathrm{~F}_{5}\right)_{3}(0.068 \mathrm{~g}, 0.132 \mathrm{mmol})$ was added resulting in the immediate precipitation of a dark red gummy solid. The reaction was stirred for $18 \mathrm{hr}$, then the liquor was decanted and discarded. The remaining material was washed with $2 \times 10 \mathrm{~mL} \mathrm{C}_{6} \mathrm{H}_{6}$ and $10 \mathrm{~mL} n$-pentane and dried to afford red solid assigned as cis-( $\left.{ }^{\text {allyl }} \mathrm{CNC}\right) \mathrm{Fe}(\mathrm{CO})_{2}\left(\mathrm{C}_{6} \mathrm{~F}_{4}-\mathrm{BF}\left(\mathrm{C}_{6} \mathrm{~F}_{5}\right)_{2}\right)$. This material is not analytically pure ( $0.102 \mathrm{~g}$ obtained); further purification attempts were unsuccessful. Single, $\mathrm{X}$-ray quality crystals were obtained by diffusing $n$-pentane into a DME solution of cis-( $\left.{ }^{\text {allyl }} \mathrm{CNC}\right) \mathrm{Fe}(\mathrm{CO})_{2}\left(\mathrm{C}_{6} \mathrm{~F}_{4}-\mathrm{BF}\left(\mathrm{C}_{6} \mathrm{~F}_{5}\right)_{2}\right)$ at room temperature. Representative spectra are provided below. Diagnostic spectroscopic features (tentative assignment): Selected IR data $(\mathrm{KBr}) \mathrm{v}=2053,1999 \mathrm{~cm}^{-1}(\mathrm{CO}) \cdot{ }^{19} \mathrm{~F} \mathrm{NMR}$ (DME, $\left.25^{\circ} \mathrm{C}, 376 \mathrm{MHz}\right) \delta$ $=-188.84$ (broad, B-F). ${ }^{11} \mathrm{~B}$ NMR (DME, $25{ }^{\circ} \mathrm{C}, 128 \mathrm{MHz}$ ) $\delta=-24.86$ (d, $J_{B, F}=90 \mathrm{~Hz}$ ). HR LC-MS of [M]-F $\left(\mathrm{C}_{37} \mathrm{H}_{17} \mathrm{~N}_{5} \mathrm{~F}_{15} \mathrm{O}_{2} \mathrm{~B}_{1} \mathrm{Fe}_{1}-\mathrm{F}\right)$ : calc. 896.0601; found 896.0599.
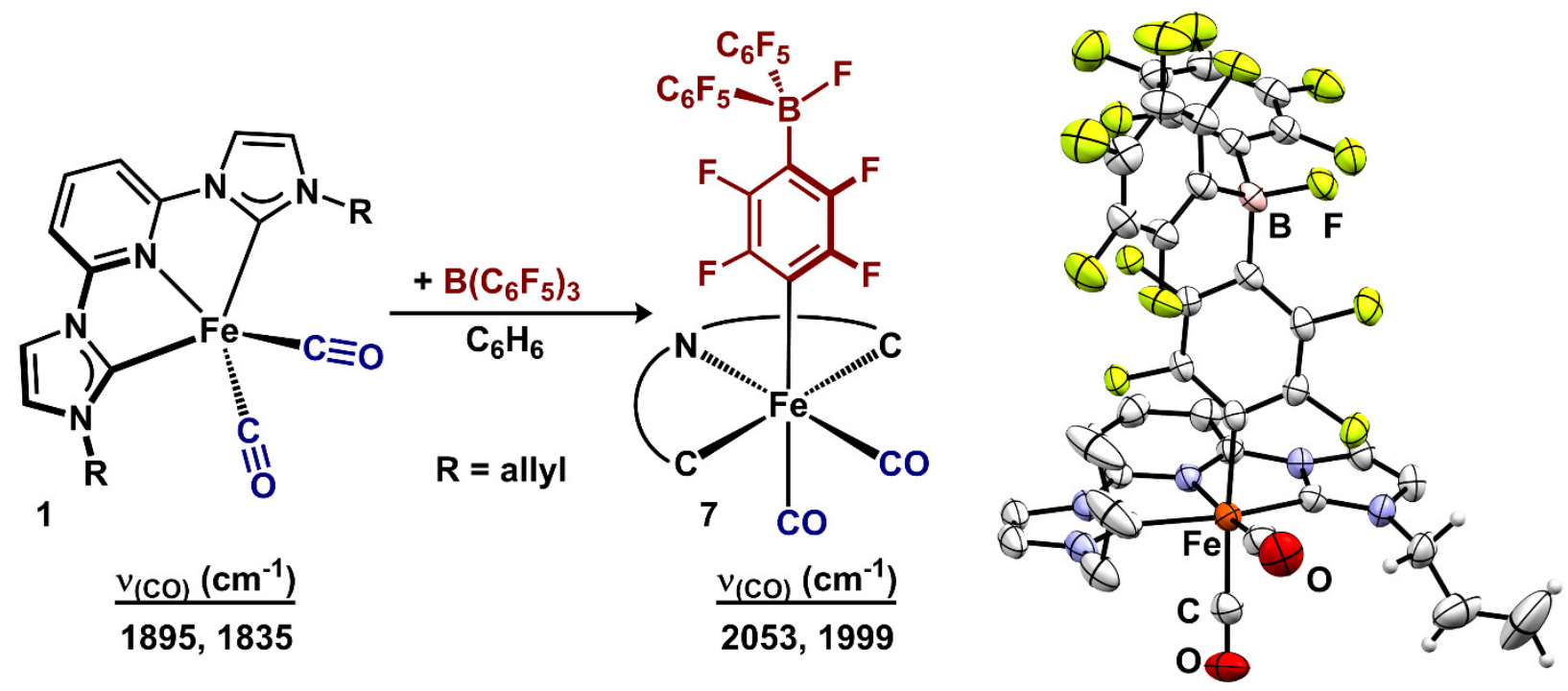

Figure S63. Reaction of 1 with a strong Lewis acid, $B\left(\mathrm{C}_{6} \mathrm{~F}_{5}\right)_{3}$, results in formation of cis(allyl $\mathrm{CNC}) \mathrm{Fe}(\mathrm{CO})_{2}\left(\mathrm{C}_{6} \mathrm{~F}_{4}-\mathrm{BF}\left(\mathrm{C}_{6} \mathrm{~F}_{5}\right)_{2}\right)$. Molecular structure of cis-(allyl $\left.\mathrm{CNC}\right) \mathrm{Fe}(\mathrm{CO})_{2}\left(\mathrm{C}_{6} \mathrm{~F}_{4}-\mathrm{BF}\left(\mathrm{C}_{6} \mathrm{~F}_{5}\right)_{2}\right)$ displayed with $50 \%$ probability ellipsoids. All $\mathrm{H}$-atoms (except those attached to one allylic moiety) are omitted for clarity. 


\section{Discussion of FLP type chemistry observed with (allyl $\mathrm{CNC}) \mathrm{Fe}(\mathrm{CO})_{2}(1)$.}

In our previous exploration of the late-stage hydroboration of $\mathbf{1}$, we did not encounter compatibility issues and the primary coordination environment at iron remained unperturbed. ${ }^{1}$ Through the course of our current study, we became interested if there was a limit whereby the primary coordination environment-and particularly the ancillary carbonyl ligands-would no longer tolerate the hydroboration conditions. To probe this question, we treated a THF solution of $\mathbf{1}$ with a potent hydroborating agent with high Lewis acidity, $\mathrm{HB}\left(\mathrm{C}_{6} \mathrm{~F}_{5}\right)_{2} \cdot{ }^{15}$ Upon addition of two equivalents of the borane, a rapid color change from dark red to deep purple was noted and the crude mixture was analyzed by NMR spectroscopy. To our surprise, the ${ }^{1} \mathrm{H}$ NMR spectrum displayed the presence of allylic resonances remaining; however, the data suggested multiple species had formed. We initially hypothesized that the highly Lewis acidic $\mathrm{HB}\left(\mathrm{C}_{6} \mathrm{~F}_{5}\right)_{2}$ may be engaging in Lewis acid/base chemistry rather than hydroboration reactivity.

To probe whether Lewis acid/base adducts were preventing the hydroboration, and to simplify our analysis, we investigated the reactivity of 1 with $\mathrm{B}\left(\mathrm{C}_{6} \mathrm{~F}_{5}\right)_{3}$ (Figure above). This strong, exogenous Lewis acid has commonly been employed for activating small molecule substrates at metals. ${ }^{16}$ Treating a red, toluene solution of 1 with a single equivalent of $B\left(\mathrm{C}_{6} \mathrm{~F}_{5}\right)_{3}$ resulted in the immediate precipitation of a purple solid which was analyzed by IR ( $\mathrm{KBr}$ ) spectroscopy. Compound cis-(allyl $\mathrm{CNC}) \mathrm{Fe}(\mathrm{CO})_{2}\left(\mathrm{C}_{6} \mathrm{~F}_{4}-\mathrm{BF}\left(\mathrm{C}_{6} \mathrm{~F}_{5}\right)_{2}\right)$ displayed two dominant $v(C O)$ stretches at 2053 and $1999 \mathrm{~cm}^{-1}$. These bands are shifted to considerably higher energy than those in the starting material $\left(1895,1835 \mathrm{~cm}^{-1}\right)$ suggesting that adduct formation of the type Fe-CO$\mathrm{B}\left(\mathrm{C}_{6} \mathrm{~F}_{5}\right)_{3}$ did not occur. We have not been able to isolate cis-(allyl $\left.\mathrm{CNC}\right) \mathrm{Fe}(\mathrm{CO})_{2}\left(\mathrm{C}_{6} \mathrm{~F}_{4}-\mathrm{BF}\left(\mathrm{C}_{6} \mathrm{~F}_{5}\right)_{2}\right)$ in analytically pure form. The molecular structure of cis-( $\left.{ }^{\text {allyl }} \mathrm{CNC}\right) \mathrm{Fe}(\mathrm{CO})_{2}\left(\mathrm{C}_{6} \mathrm{~F}_{4}-\mathrm{BF}\left(\mathrm{C}_{6} \mathrm{~F}_{5}\right)_{2}\right)$ was determined by analysis of single crystals obtained from 1,2-dimethoxyethane solution and revealed the formation of a new Fe- $C$ bond $(2.071(3) \AA)$ via formal C-F oxidative addition at the para-position of the Lewis acid to form cis(allyl $\mathrm{CNC}) \mathrm{Fe}(\mathrm{CO})_{2}\left(\mathrm{C}_{6} \mathrm{~F}_{4}-\mathrm{BF}\left(\mathrm{C}_{6} \mathrm{~F}_{5}\right)_{2}\right)$.

The result of the addition of $\mathrm{B}\left(\mathrm{C}_{6} \mathrm{~F}_{5}\right)_{3}$ to 1 is net $\mathrm{C}-\mathrm{F}$ oxidative addition to form cis- $\left({ }^{\text {allyl }} \mathrm{CNC}\right) \mathrm{Fe}(\mathrm{CO})_{2}\left(\mathrm{C}_{6} \mathrm{~F}_{4^{-}}\right.$ $\left.B F\left(C_{6} F_{5}\right)_{2}\right)$. The fluoride is captured by the Lewis acidic borane $\left(B-F=1.444(3) \AA ; \Sigma B \alpha=323.5(2)^{\circ}\right)$. An analogous cooperative $\mathrm{C}-\mathrm{F}$ bond activation was demonstrated with homobimetallic $\left[\mathrm{MN}\left(\mathrm{P}^{i} \mathrm{Pr}_{2}\right)_{2}\right]_{2}$ complexes $(\mathrm{M}=\mathrm{Sn}, \mathrm{Ge})^{17}$ and mirrors aromatic substitution reactions described in the context of frustrated Lewis pairs. ${ }^{18}$ The new Fe-aryl bond (2.071(3) $\AA$ ) is the longest of the five iron-carbon bonds within the molecule. The $\mathrm{Fe}-\mathrm{C}_{\mathrm{co}}$ distance of the carbonyl that is trans to the new aryl ligand is considerably longer (1.814(3) $\AA$ ) than its cis counterpart (1.769(3) $\AA$ ), while the Fe- $C_{C N C}$ distances (1.967(3), 1.969(2) $\AA$ ) are typical of low-spin octahedral iron complexes of this type. ${ }^{19}$ 


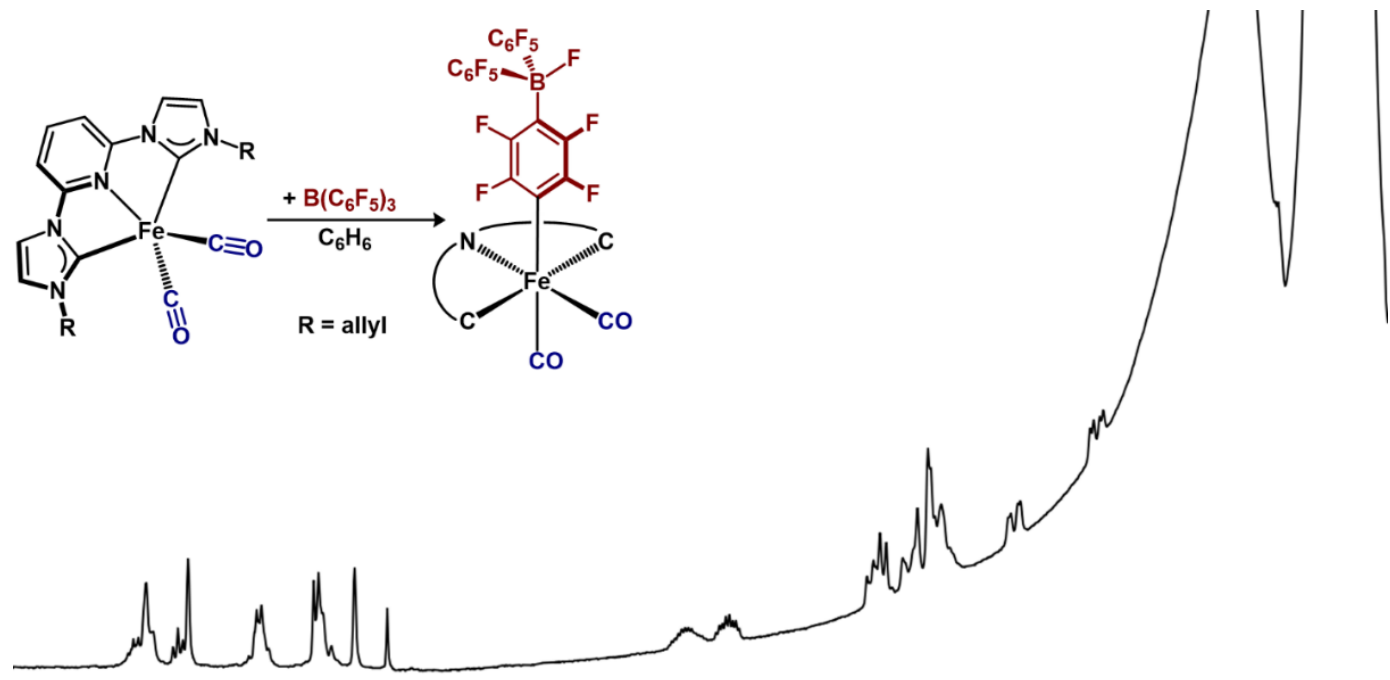

8.8 8.6 8.4 8.2 8.0 7.8 7.6 7.4 7.2 7.0 6.8 6.6 6.4 6.2 6.0 5.8 5.6 5.4 5.2 5.0 4.8 4.6 4.4 4.2 4.0 3.8 3.6 3.4 ppm

Figure S64 ${ }^{1} \mathrm{H}$ NMR spectrum (DME, $25{ }^{\circ} \mathrm{C}, 400 \mathrm{MHz}$ ) of isolated material from reaction between $\left({ }^{\text {allyl }} \mathrm{CNC}\right) \mathrm{Fe}(\mathrm{CO})_{2}(\mathbf{1})$ and $\mathrm{B}\left(\mathrm{C}_{6} \mathrm{~F}_{5}\right)_{3}$.
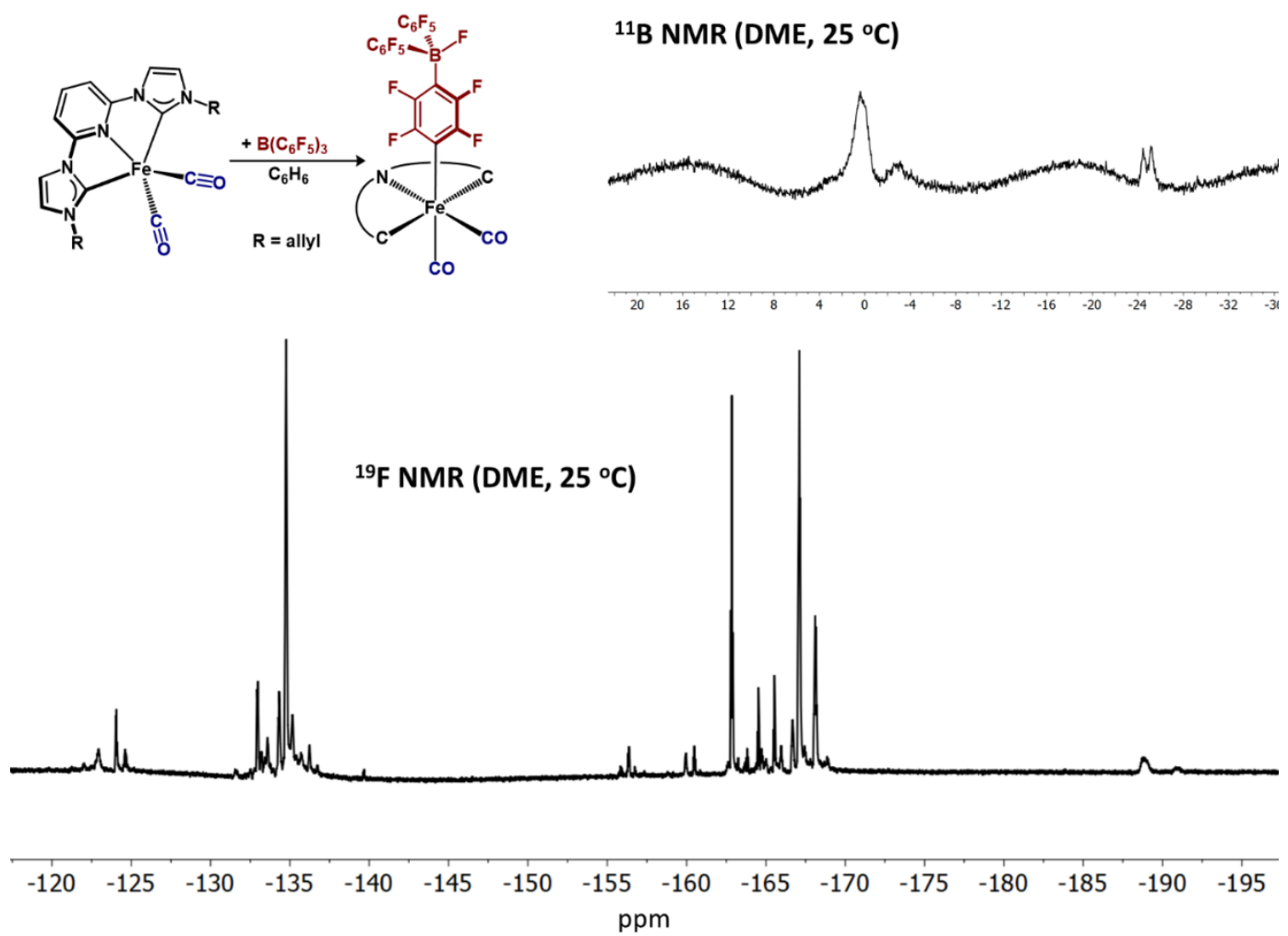

Figure $\mathrm{S} 65{ }^{19} \mathrm{~F}$ NMR spectrum (DME, $25{ }^{\circ} \mathrm{C}, 376 \mathrm{MHz}$ ) of isolated material from reaction between $\left({ }^{\text {allyl }} \mathrm{CNC}\right) \mathrm{Fe}(\mathrm{CO})_{2}(\mathbf{1})$ and $\mathrm{B}\left(\mathrm{C}_{6} \mathrm{~F}_{5}\right)_{3}$. The inset displays the corresponding ${ }^{11} \mathrm{~B} N \mathrm{NMR}$ spectrum $\left(\mathrm{DME}, 25{ }^{\circ} \mathrm{C}, 128\right.$ $\mathrm{MHz}$ ). 


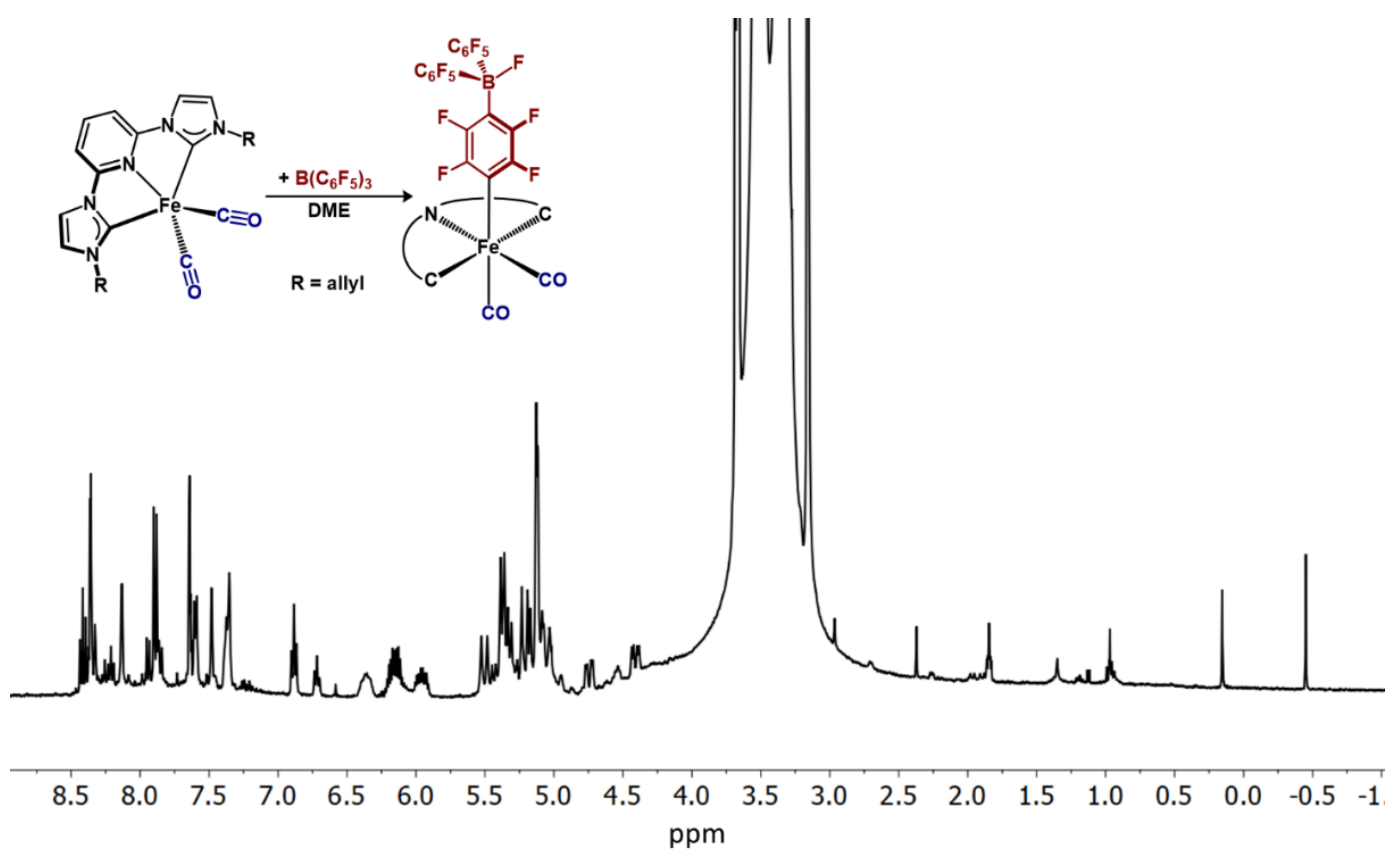

Figure S66 In situ ${ }^{1} \mathrm{H}$ NMR spectrum (DME, $25{ }^{\circ} \mathrm{C}, 400 \mathrm{MHz}$ ) from reaction between $\left({ }^{\text {allyl }} \mathrm{CNC}\right) \mathrm{Fe}(\mathrm{CO})_{2}(\mathbf{1})$ and $\mathrm{B}\left(\mathrm{C}_{6} \mathrm{~F}_{5}\right)_{3}$. 


\section{Infrared Spectra}

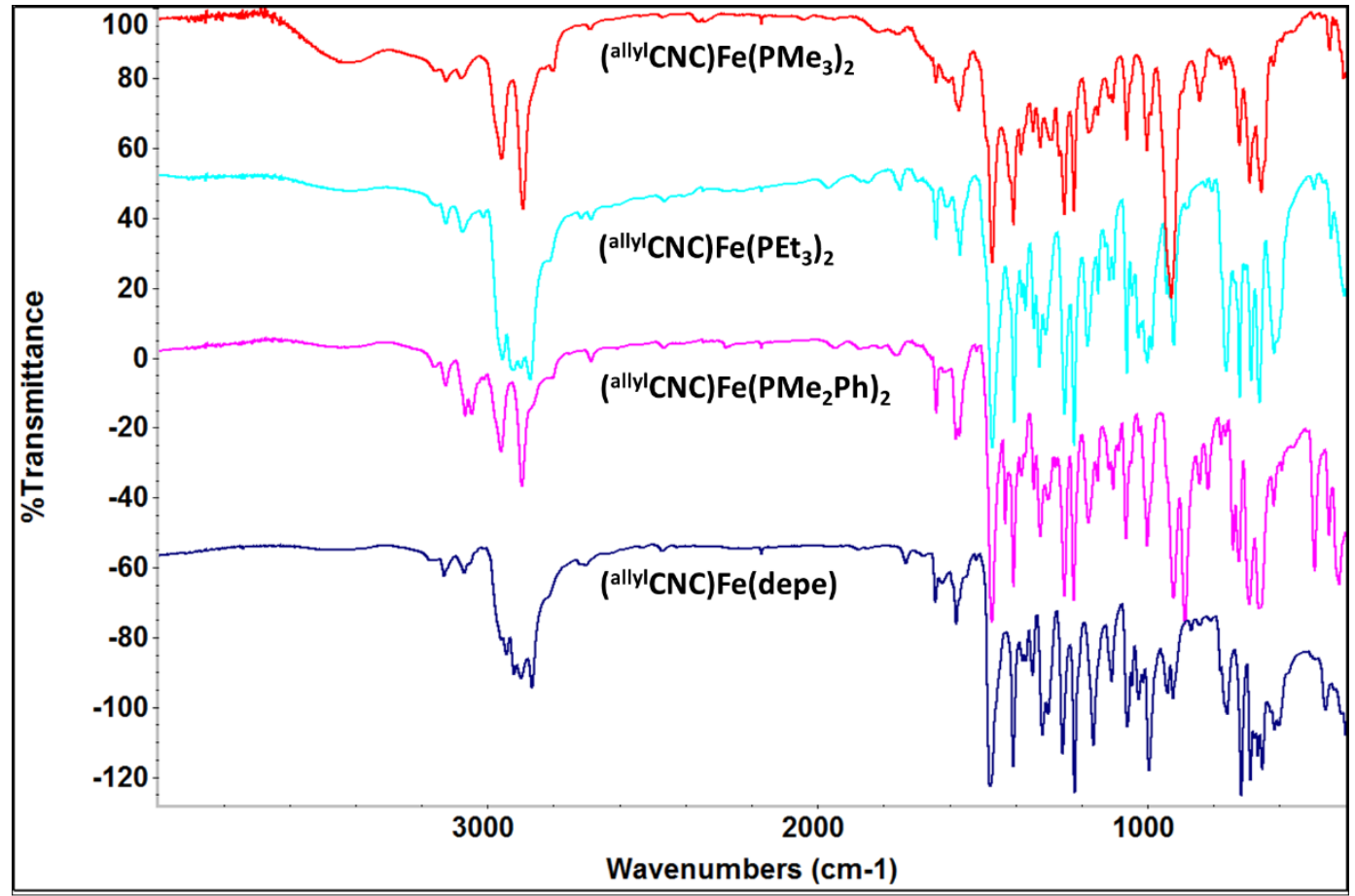

Figure $\mathbf{S 6 7}$ Infrared spectra ( $\mathrm{KBr}$ ), from top to bottom, of (allyl $\mathrm{CNC}) \mathrm{Fe}\left(\mathrm{PMe}_{3}\right)_{2}\left(3-\mathrm{PMe}_{3}\right),\left({ }^{\text {allyl }} \mathrm{CNC}\right) \mathrm{Fe}(\mathrm{PEt})_{2}$ (3-PEt 3 ), ( $\left.{ }^{\text {allyl }} \mathrm{CNC}\right) \mathrm{Fe}\left(\mathrm{PMe}{ }_{2} \mathrm{Ph}\right)_{2}$ (3-PMe $\left.2 \mathrm{Ph}\right)$, and (allyl $\left.\mathrm{CNC}\right) \mathrm{Fe}($ depe) (3-depe).

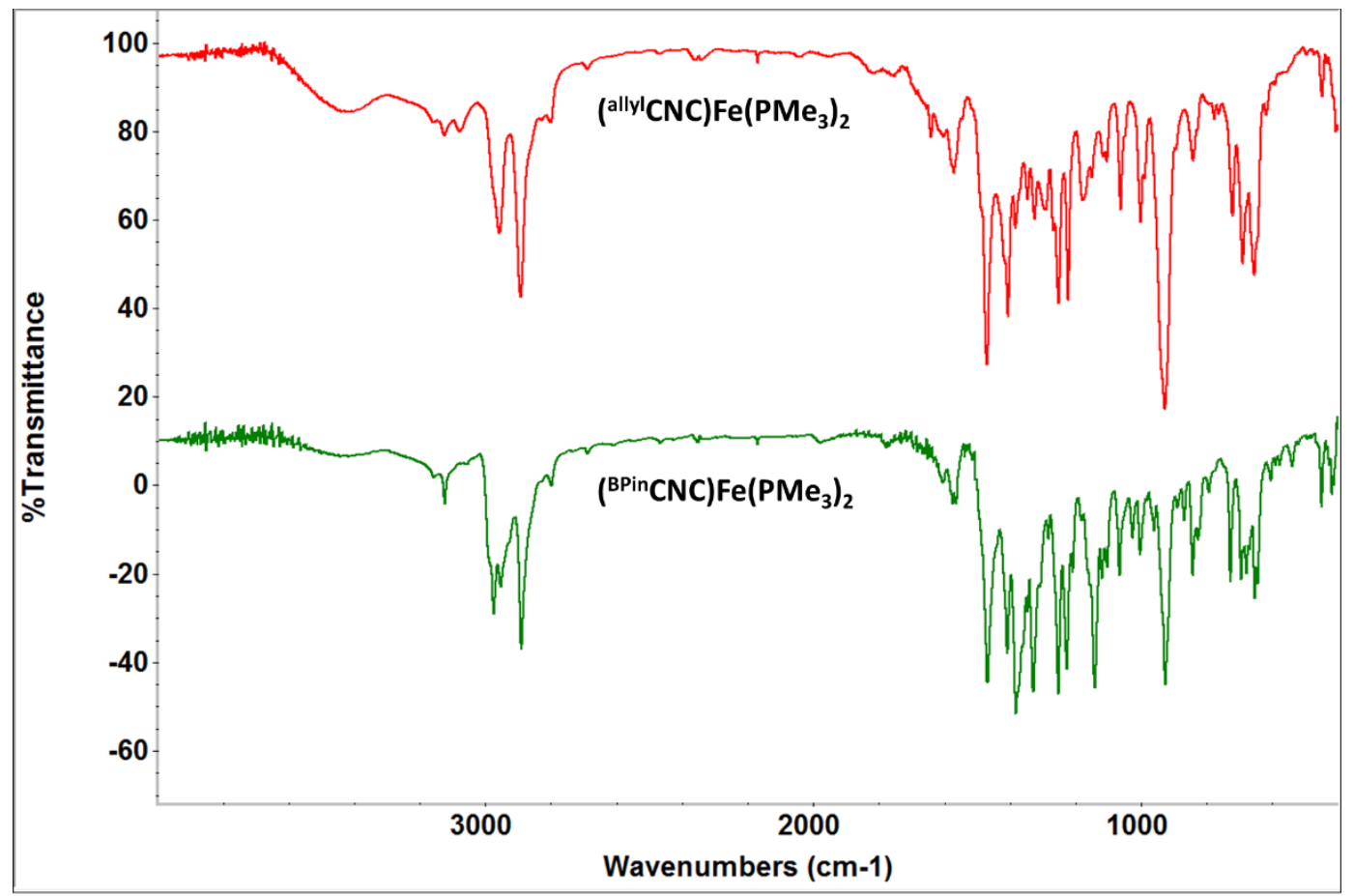

Figure S68 Infrared spectra (KBr) of ( $\left.{ }^{\text {allyl }} \mathrm{CNC}\right) \mathrm{Fe}\left(\mathrm{PMe}_{3}\right)_{2}\left(3-\mathrm{PMe}_{3}\right)$ (top) and ( $\left.{ }^{\mathrm{BPin}} \mathrm{CNC}\right) \mathrm{Fe}\left(\mathrm{PMe}_{3}\right)_{2}\left(6-\mathrm{PMe}_{3}\right)$ (bottom). 


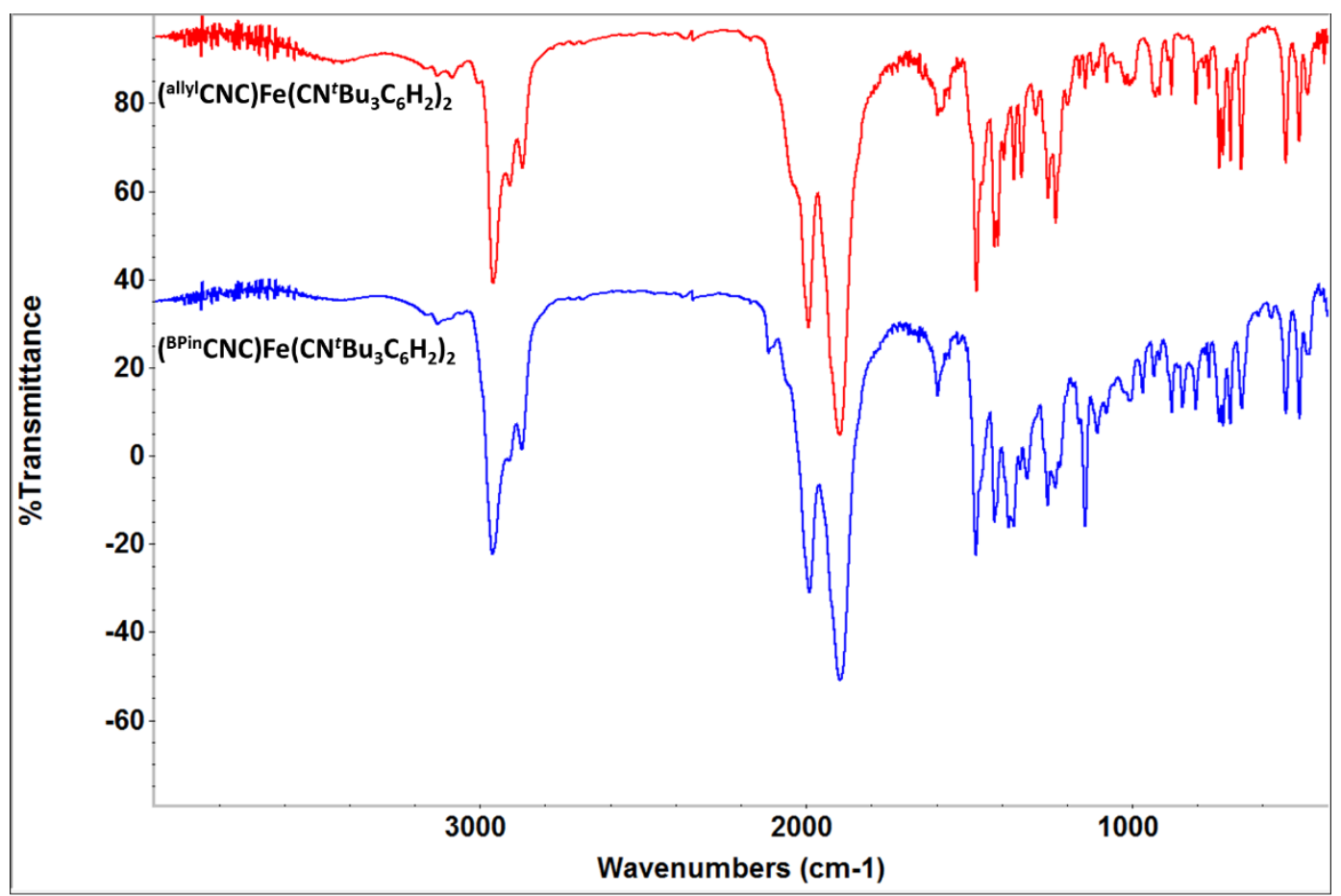

Figure S69 Infrared spectra $(\mathrm{KBr})$ of $\quad\left({ }^{\text {allyl }} \mathrm{CNC}\right) \mathrm{Fe}\left(\mathrm{CNN}^{t} \mathrm{Bu}_{3} \mathrm{C}_{6} \mathrm{H}_{2}\right)_{2} \quad\left(4_{-}{ }^{t} \mathrm{Bu}_{3} \mathrm{C}_{6} \mathrm{H}_{2}\right)$ (top) and $\left({ }^{B P i n} \mathrm{CNC}\right) \mathrm{Fe}\left(\mathrm{CN}^{t} \mathrm{Bu}_{3} \mathrm{C}_{6} \mathrm{H}_{2}\right)_{2}\left(6-\mathrm{CN}^{t} \mathrm{Bu}_{3} \mathrm{C}_{6} \mathrm{H}_{2}\right)$ (bottom).

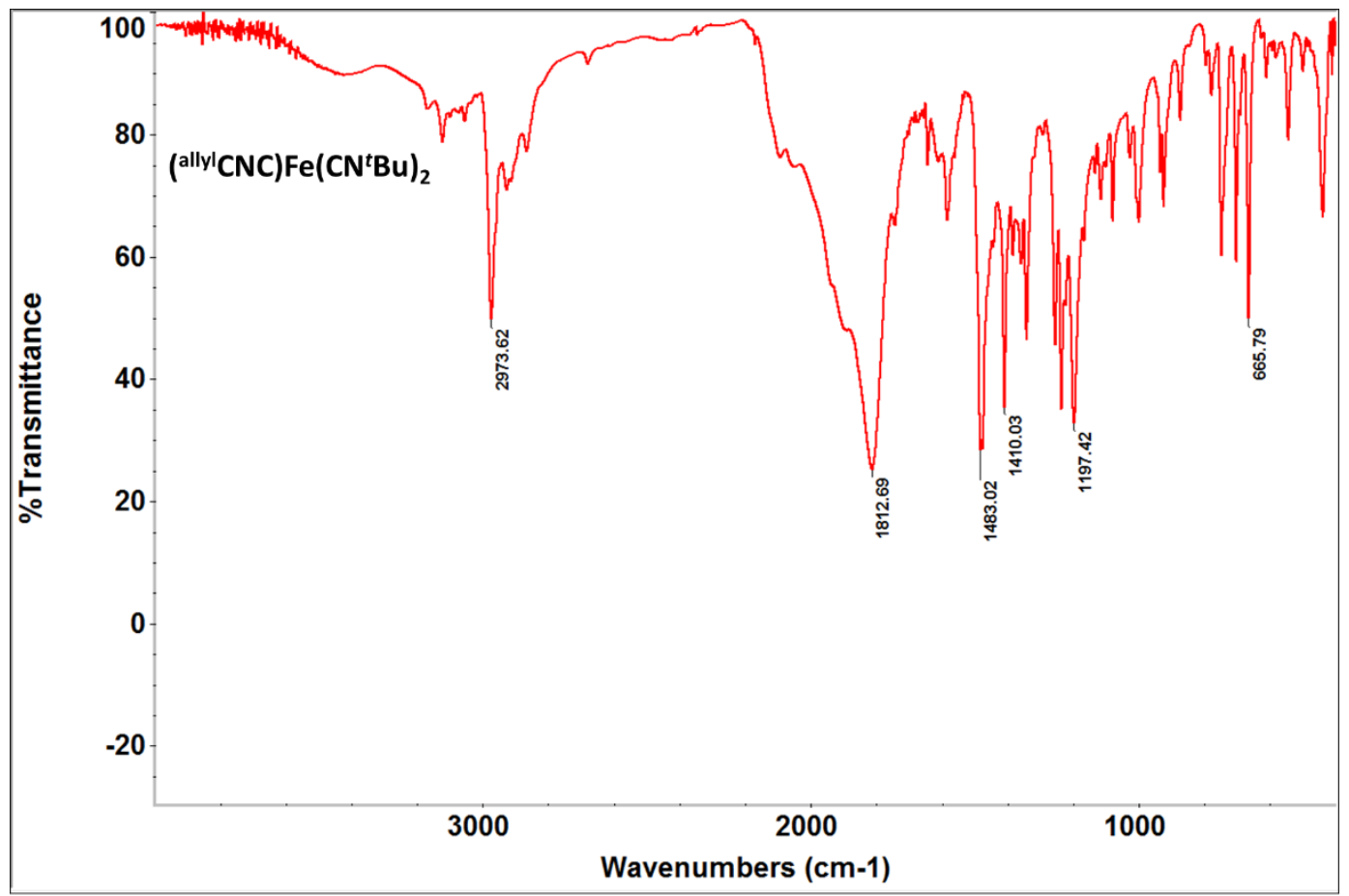

Figure S70 Infrared spectrum $(\mathrm{KBr})$ of $\left({ }^{\text {allyl }} \mathrm{CNC}\right) \mathrm{Fe}\left(\mathrm{CN}^{t} \mathrm{Bu}\right)_{2}\left(4^{-}{ }^{\mathrm{B} u}\right)$. 


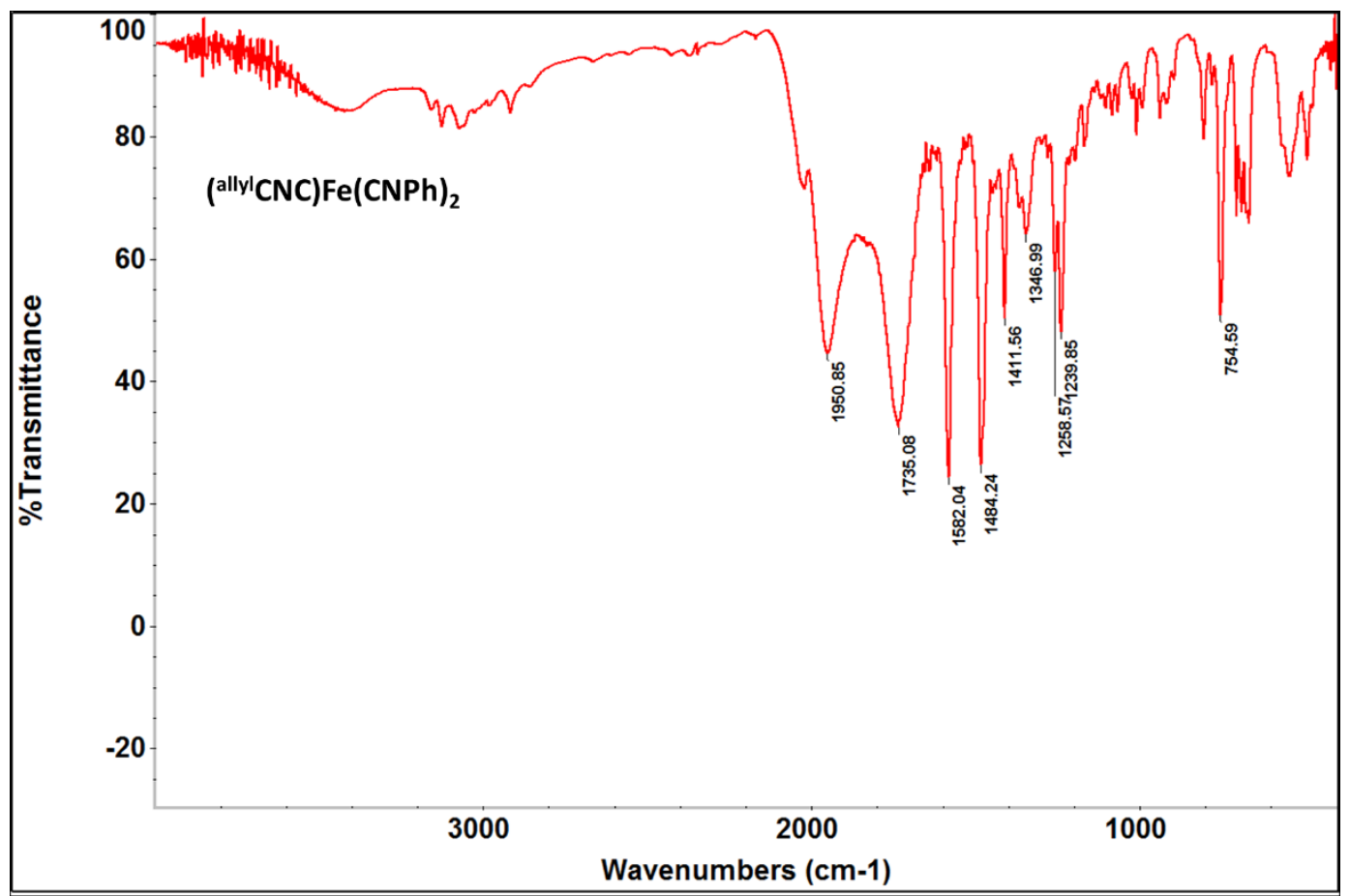

Figure S71 Infrared spectrum (KBr) of ( $\left.{ }^{\text {allyl }} \mathrm{CNC}\right) \mathrm{Fe}(\mathrm{CNPh})_{2}(4-\mathrm{Ph})$.

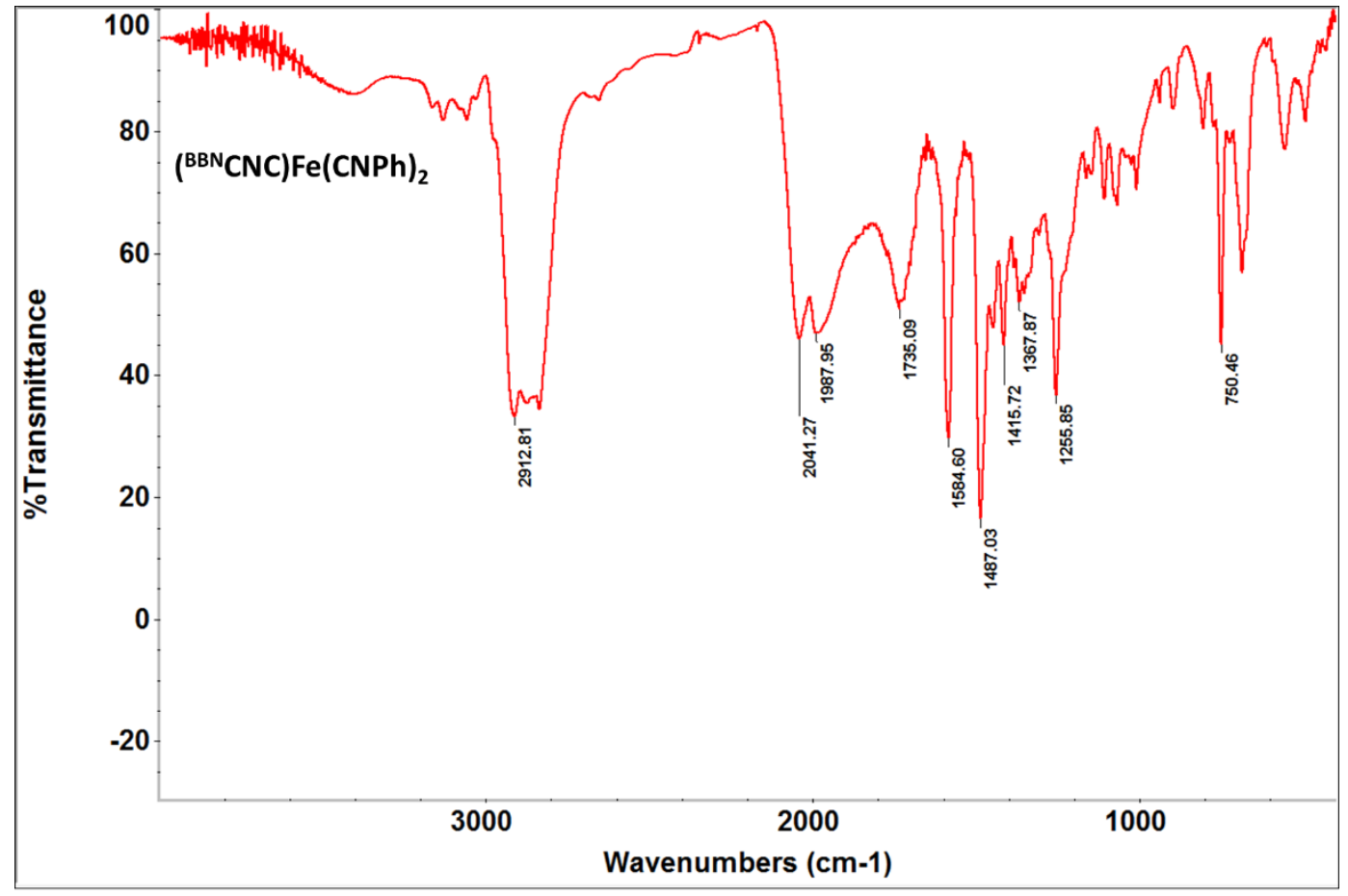

Figure S72 Infrared spectrum $(\mathrm{KBr})$ of $\left({ }^{\mathrm{BBN}} \mathrm{CNC}\right) \mathrm{Fe}(\mathrm{CNPh})_{2}(\mathbf{5}-\mathrm{Ph})$. 


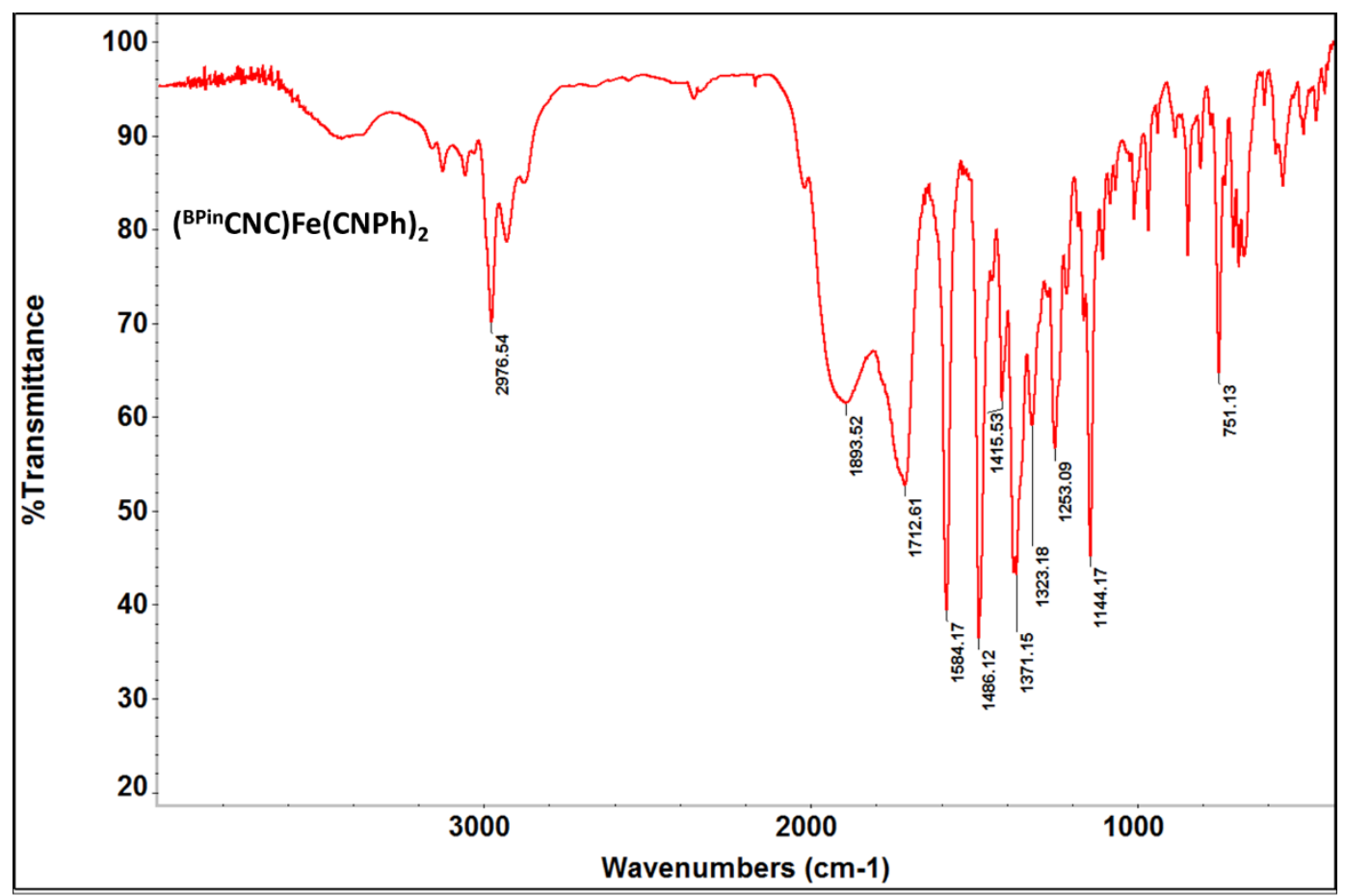

Figure S73 Infrared spectrum $(\mathrm{KBr})$ of $\left({ }^{\mathrm{BPin}} \mathrm{CNC}\right) \mathrm{Fe}(\mathrm{CNPh})_{2}(6-\mathrm{CNPh})$.

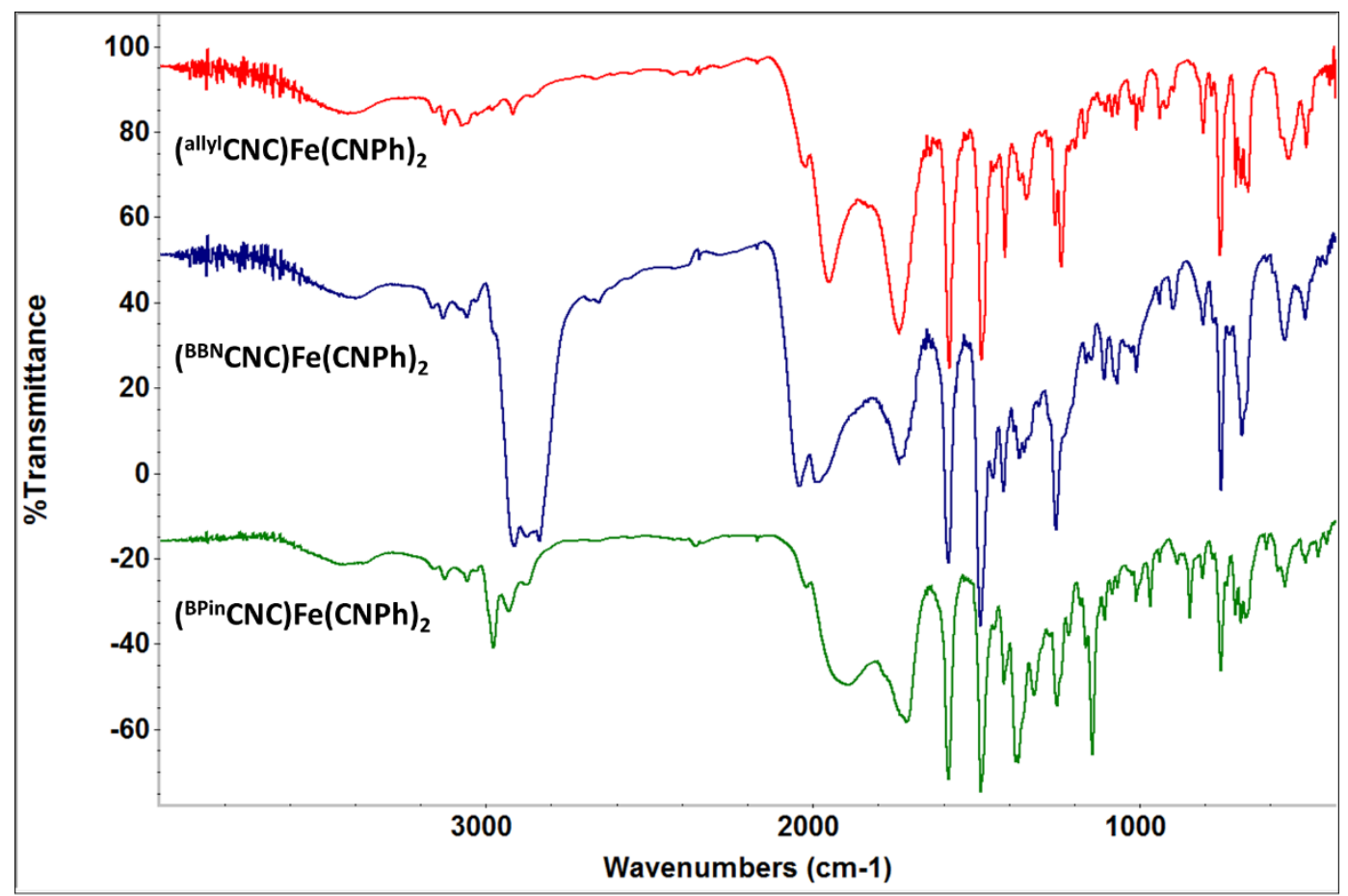

Figure S74 Infrared spectra (KBr), from top to bottom, of ( $\left.{ }^{\text {allyl }} \mathrm{CNC}\right) \mathrm{Fe}(\mathrm{CNPh})_{2}(4-\mathrm{Ph}),\left({ }^{\mathrm{BBN}} \mathrm{CNC}\right) \mathrm{Fe}(\mathrm{CNPh})_{2}(5-$ $\mathrm{Ph})$, and $\left({ }^{\mathrm{BPin}} \mathrm{CNC}\right) \mathrm{Fe}(\mathrm{CNPh})_{2}(6-\mathrm{CNPh})$. 


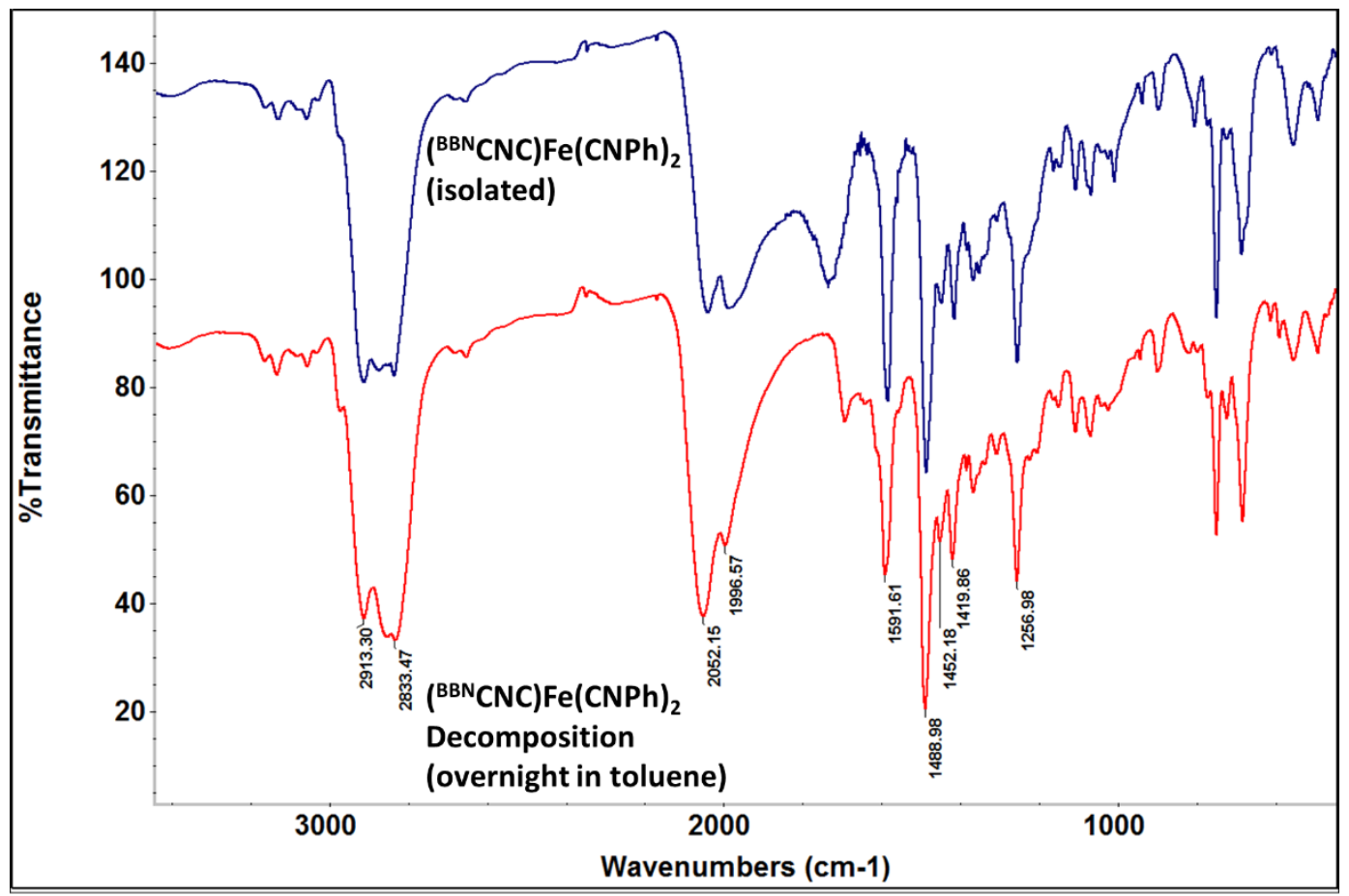

Figure S75 Infrared spectra $(\mathrm{KBr})$ of $\left({ }^{\mathrm{BBN}} \mathrm{CNC}\right) \mathrm{Fe}(\mathrm{CNPh})_{2}$ (5-Ph) (top) and its decomposition product (bottom).

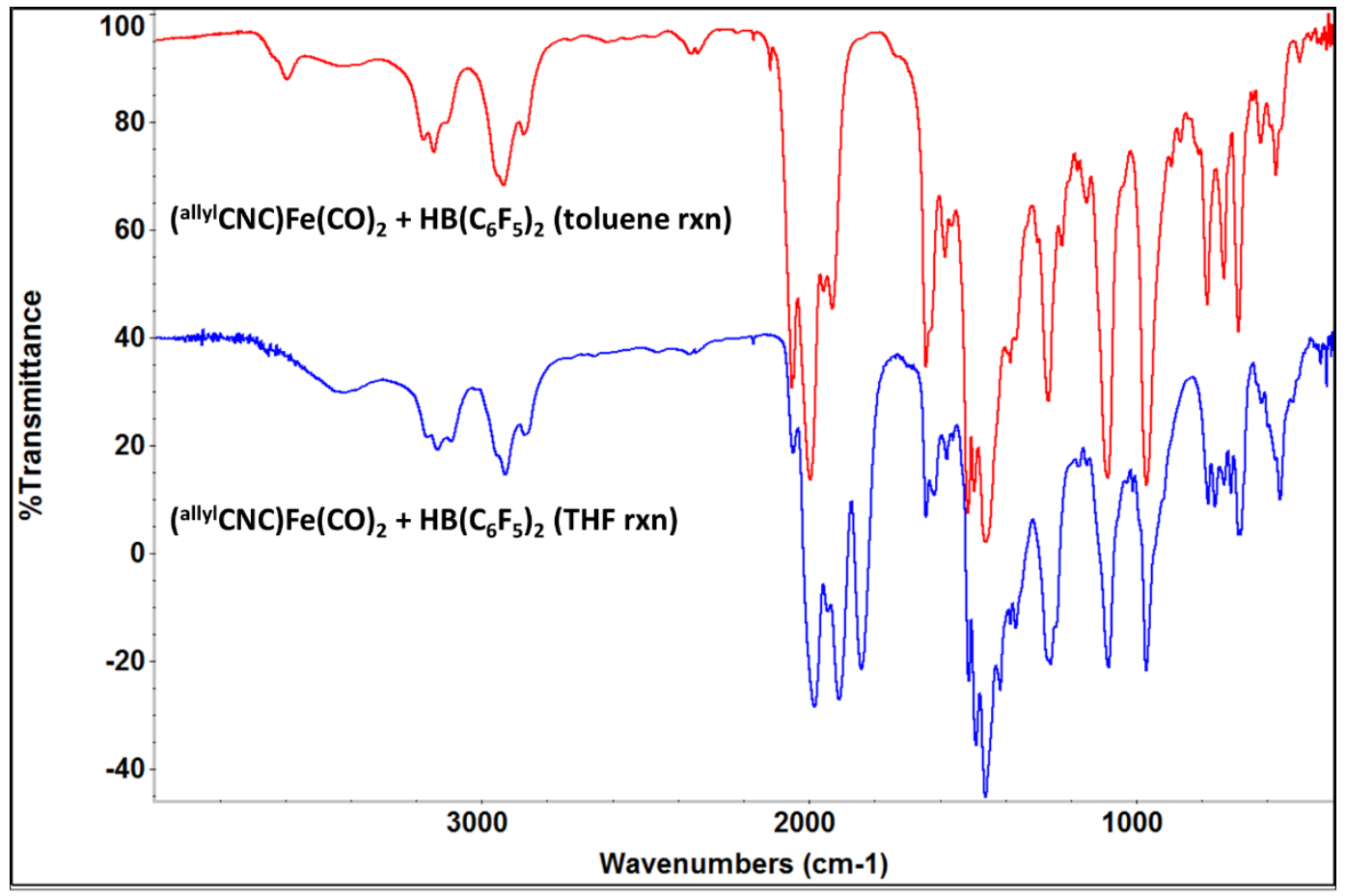

Figure S76 Infrared spectra $(\mathrm{KBr})$ of the reaction between $\left({ }^{\text {allyl }} \mathrm{CNC}\right) \mathrm{Fe}(\mathrm{CO})_{2}(\mathbf{1})$ and $\mathrm{HB}\left(\mathrm{C}_{6} \mathrm{~F}_{5}\right)_{2}$ carried out in toluene (top) and THF (bottom). 


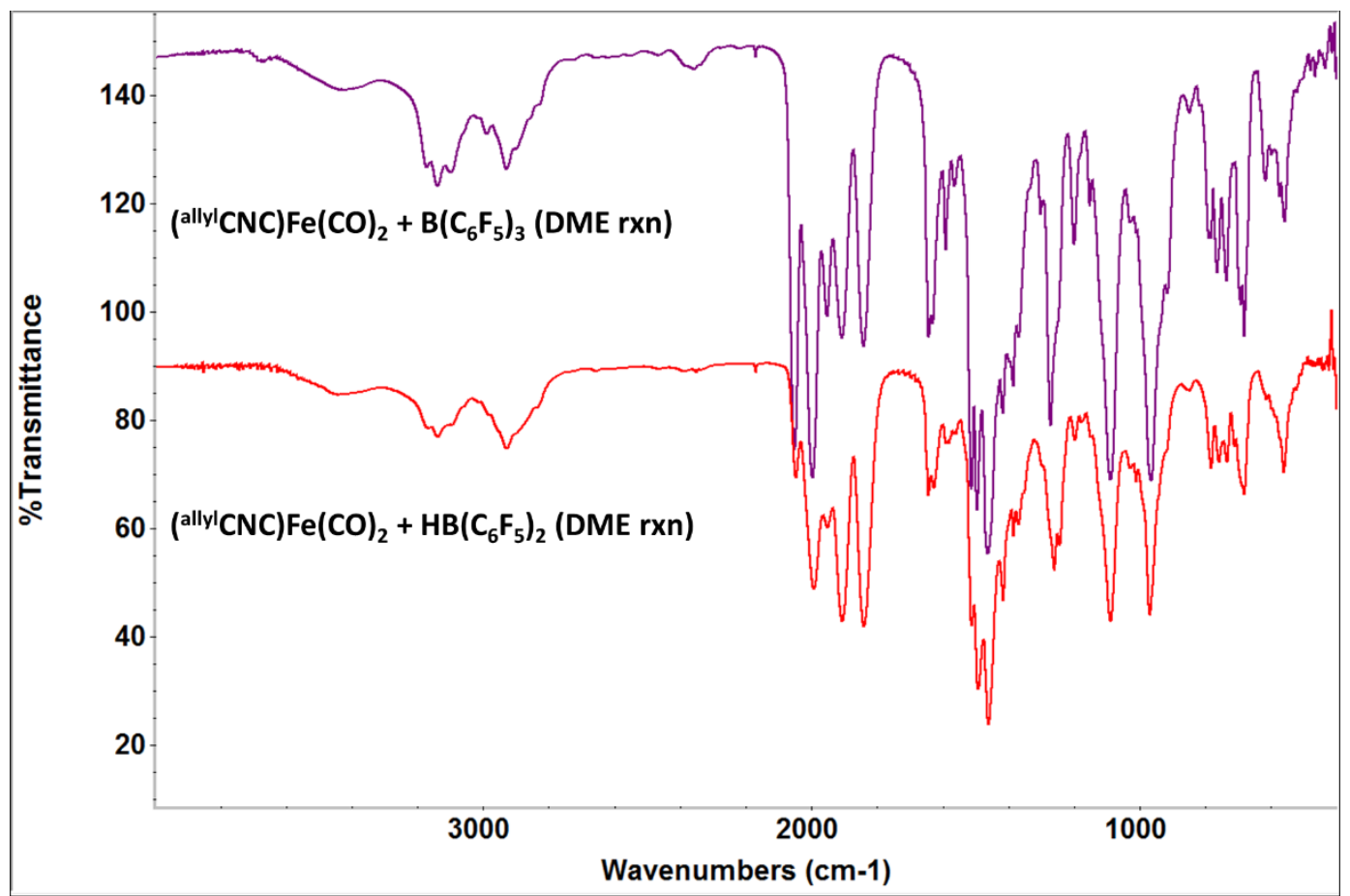

Figure S77 Infrared spectra ( $\mathrm{KBr}$ ) comparing the reactions between (allyl $\mathrm{CNC}) \mathrm{Fe}(\mathrm{CO})_{2}(\mathbf{1})$ and $\mathrm{B}\left(\mathrm{C}_{6} \mathrm{~F}_{5}\right)_{3}$ (top) vs (allyl $\mathrm{CNC}$ ) Fe(CO) $)_{2}(\mathbf{1})$ and $\mathrm{HB}\left(\mathrm{C}_{6} \mathrm{~F}_{5}\right)_{2}$ (bottom). Both reactions were performed in $\mathrm{DME}$ and the crude mixtures were analyzed.

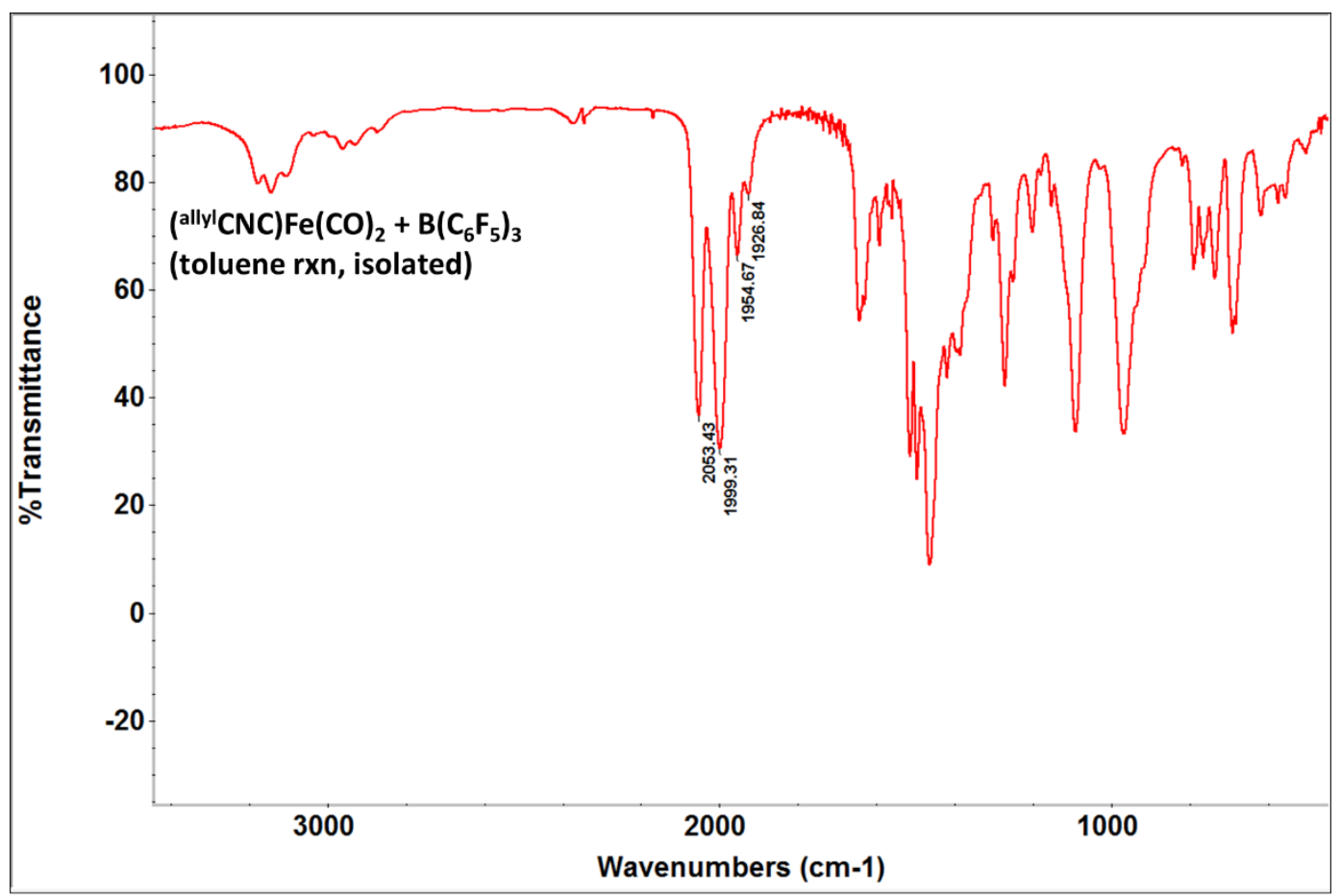

Figure S78 Infrared spectrum ( $\mathrm{KBr}$ ) of the reaction between ( $\left.{ }^{\mathrm{allyl}} \mathrm{CNC}\right) \mathrm{Fe}(\mathrm{CO})_{2}(\mathbf{1})$ and $\mathrm{B}\left(\mathrm{C}_{6} \mathrm{~F}_{5}\right)_{3}$ in toluene to generate cis-(allyl $\mathrm{CNC}) \mathrm{Fe}(\mathrm{CO})_{2}\left(\mathrm{C}_{6} \mathrm{~F}_{4}-\mathrm{BF}\left(\mathrm{C}_{6} \mathrm{~F}_{5}\right)_{2}\right)$. The material isolated by this method is not analytically pure. 


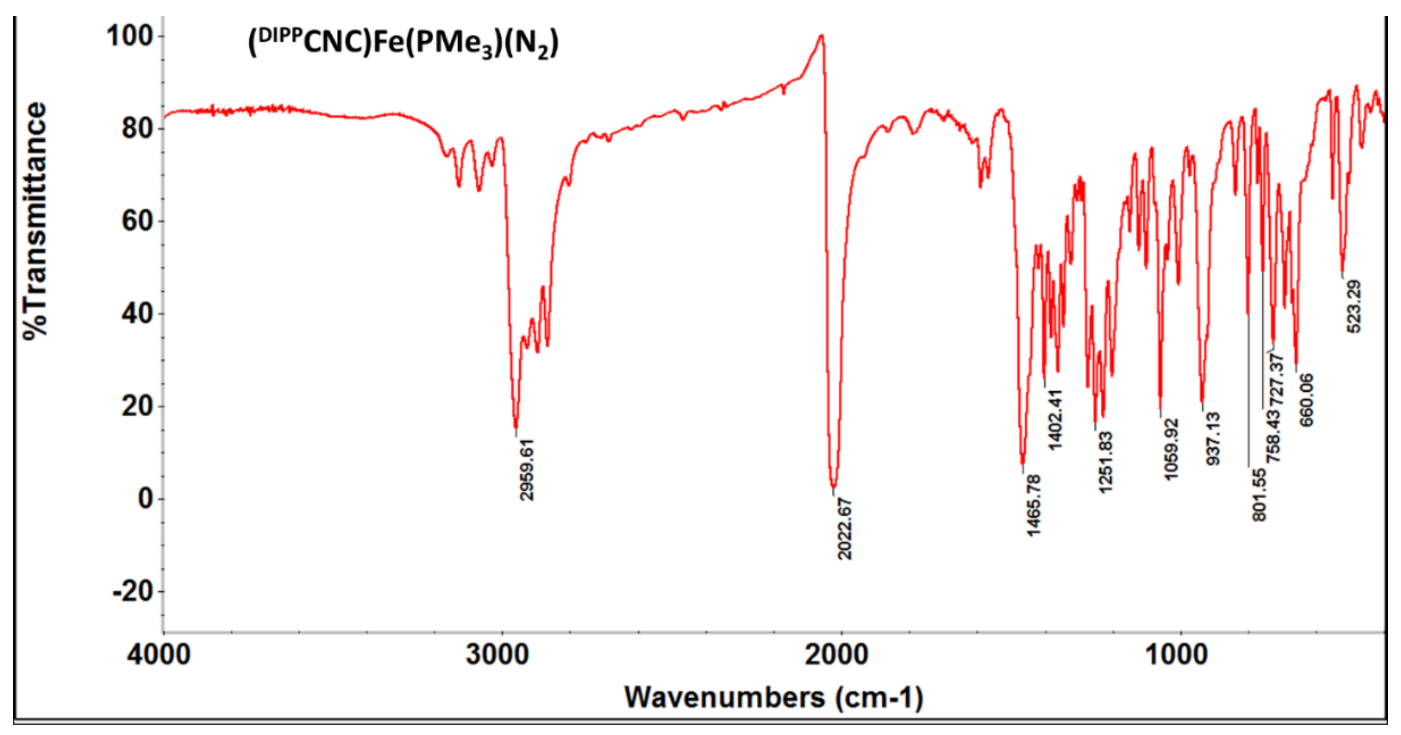

Figure $\mathbf{S 7 9}$ Infrared spectrum $(\mathrm{KBr})$ of $\left({ }^{\mathrm{DIPP}} \mathrm{CNC}\right) \mathrm{Fe}\left(\mathrm{N}_{2}\right)\left(\mathrm{PMe}_{3}\right)$.

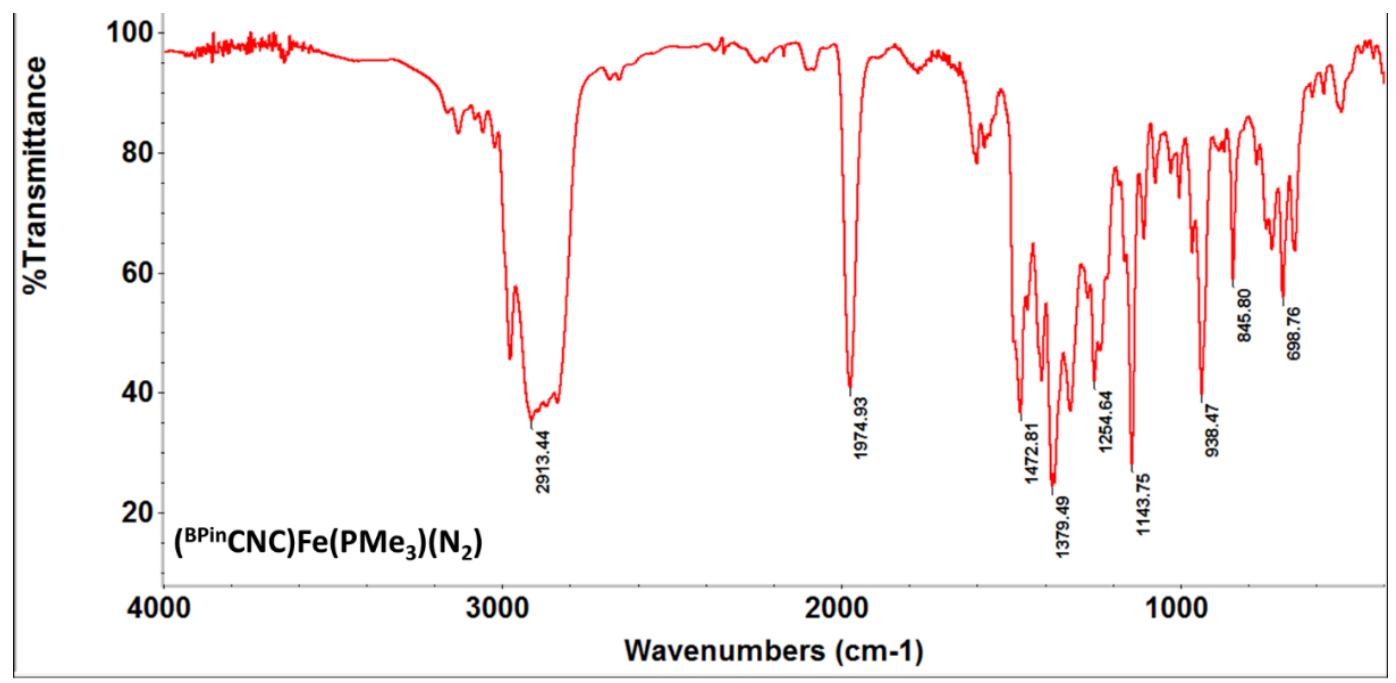

Figure $\mathbf{S 8 0}$ Infrared spectrum $(\mathrm{KBr})$ of $\left({ }^{\mathrm{BPin}} \mathrm{CNC}\right) \mathrm{Fe}\left(\mathrm{N}_{2}\right)\left(\mathrm{PMe}_{3}\right)$. This material was obtained as described above. 


\section{Electronic Absorption Spectra}

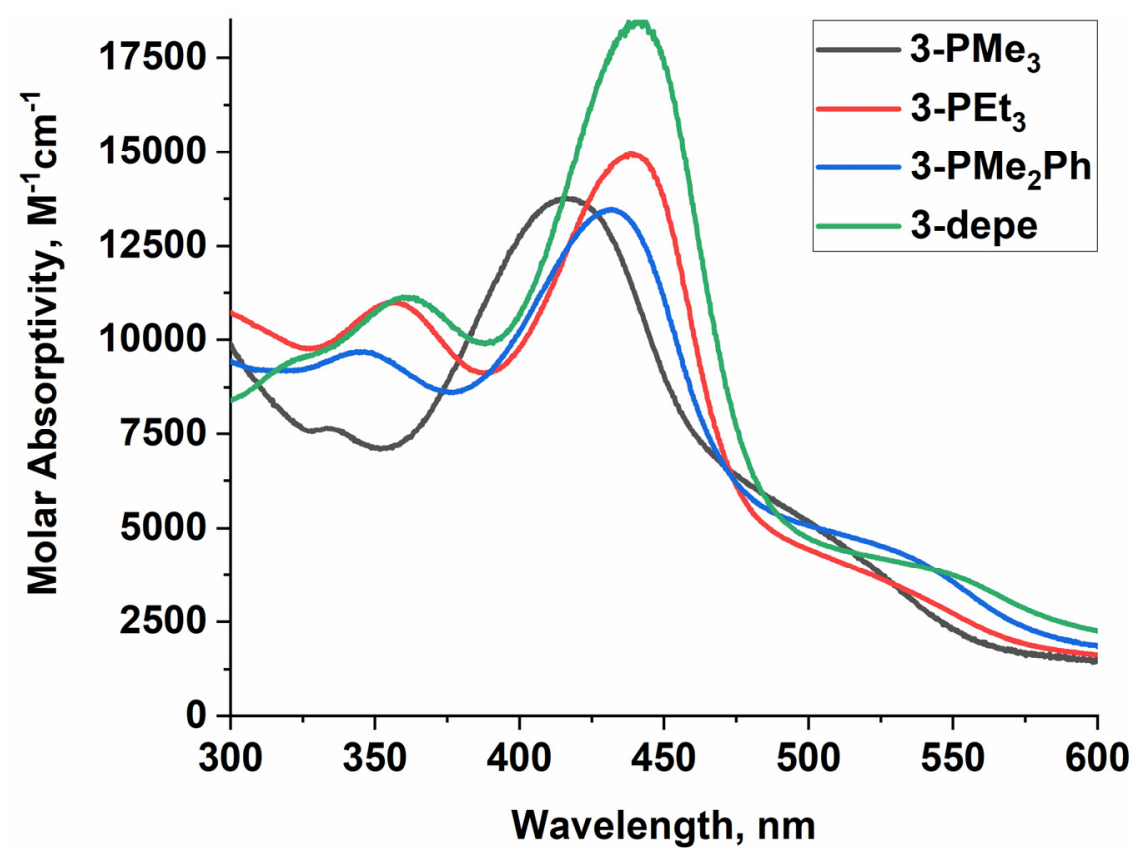

Figure S81 Electronic absorption spectra of ( $\left.{ }^{\text {allyl }} \mathrm{CNC}\right) \mathrm{Fe}\left(\mathrm{PR}_{3}\right)_{2}$ (3) $\left(\mathrm{PR}_{3}=\mathrm{PMe}_{3}, \mathrm{PEt}_{3}, \mathrm{PMe}_{2} \mathrm{Ph}, 1 / 2\right.$ depe) recorded in THF at ambient temperature.

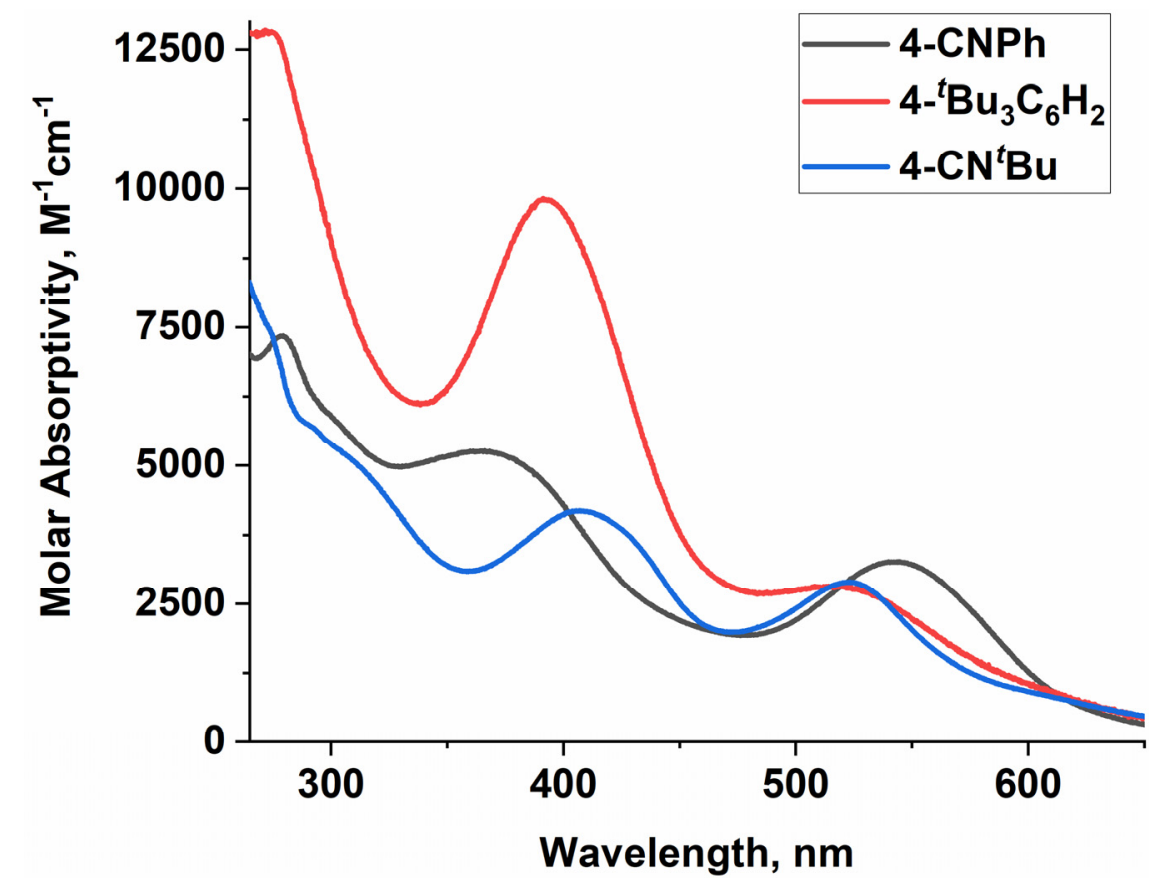

Figure S82 Electronic absorption spectra of $\left({ }^{\text {allyl }} \mathrm{CNC}\right) \mathrm{Fe}(\mathrm{CNR})_{2}(4)\left(\mathrm{CNR}=\mathrm{CN}^{t} \mathrm{Bu}, \mathrm{CNPh}, \mathrm{CN}^{t} \mathrm{Bu}_{3} \mathrm{Ph}\right)$ recorded in THF at ambient temperature. 


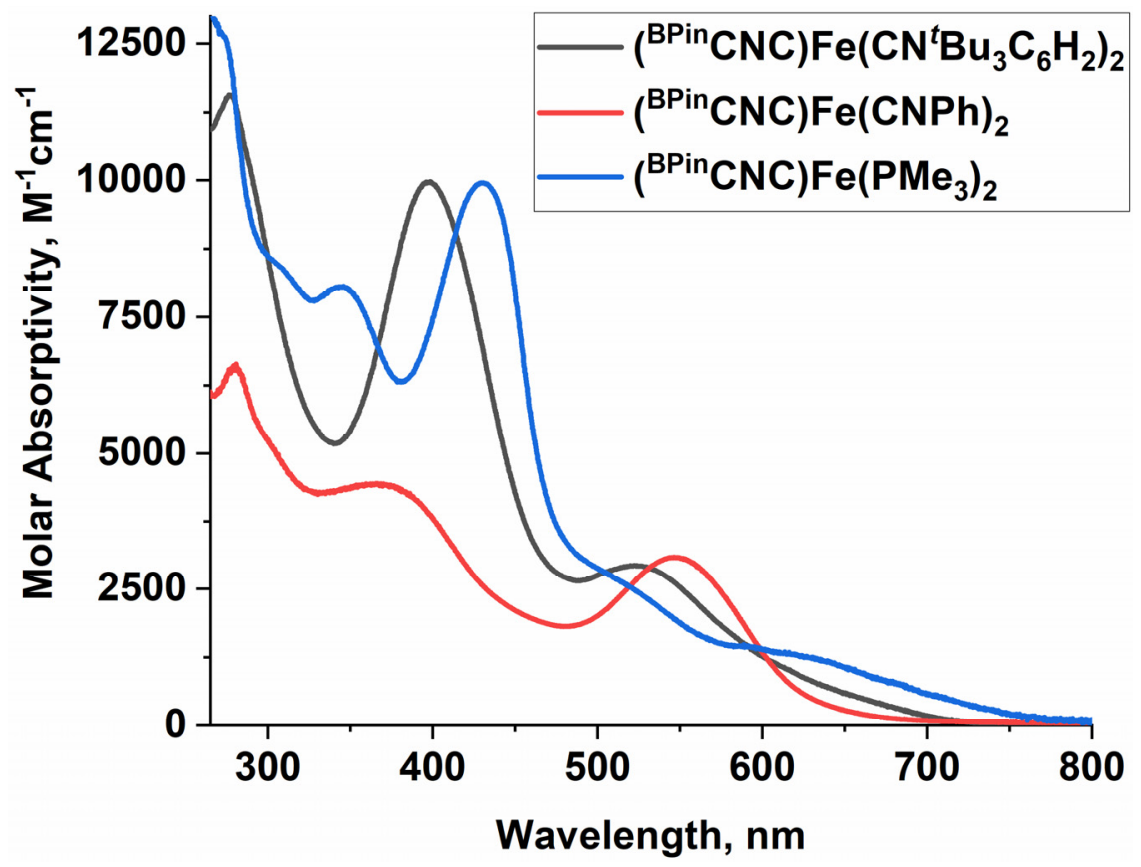

Figure S83 Electronic absorption spectra of $\left({ }^{\mathrm{BPin}} \mathrm{CNC}\right) \mathrm{Fe}(\mathrm{L})_{2}(6)\left(\mathrm{L}=\mathrm{CN}^{t} \mathrm{Bu}_{3} \mathrm{Ph}, \mathrm{CNPh}, \mathrm{PMe}_{3}\right)$ recorded in $\mathrm{THF}$ at ambient temperature.

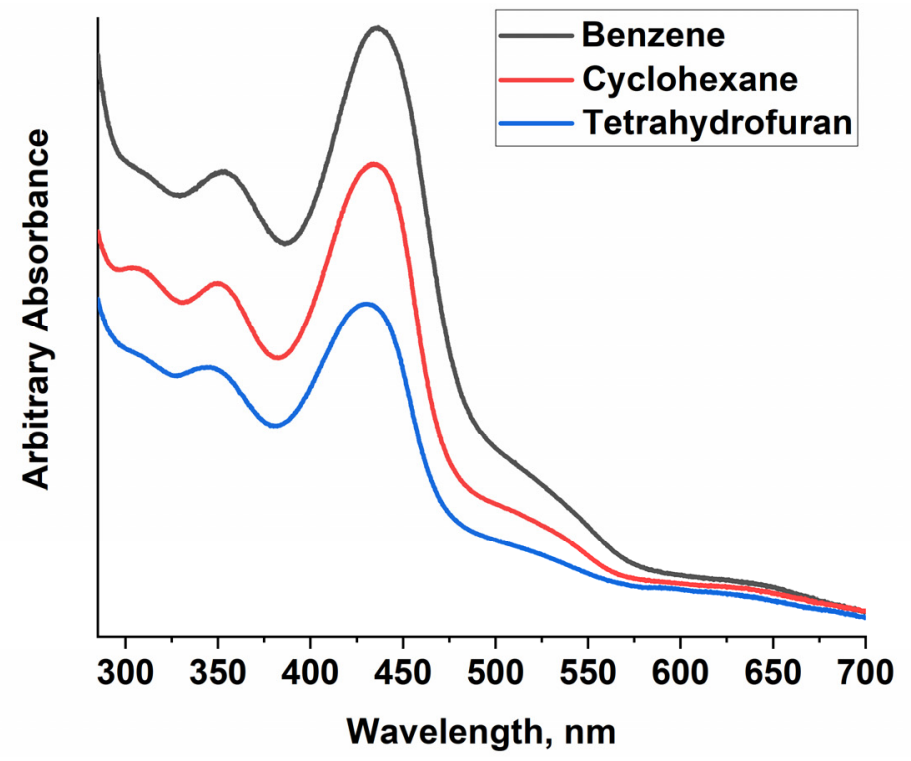

Figure S84 Electronic absorption spectra of $\left({ }^{\mathrm{BPin}} \mathrm{CNC}\right) \mathrm{Fe}\left(\mathrm{PMe}_{3}\right)_{2}$ (6-PMe $)$ recorded in benzene (black), cyclohexane (red), and THF (blue) at ambient temperature. 


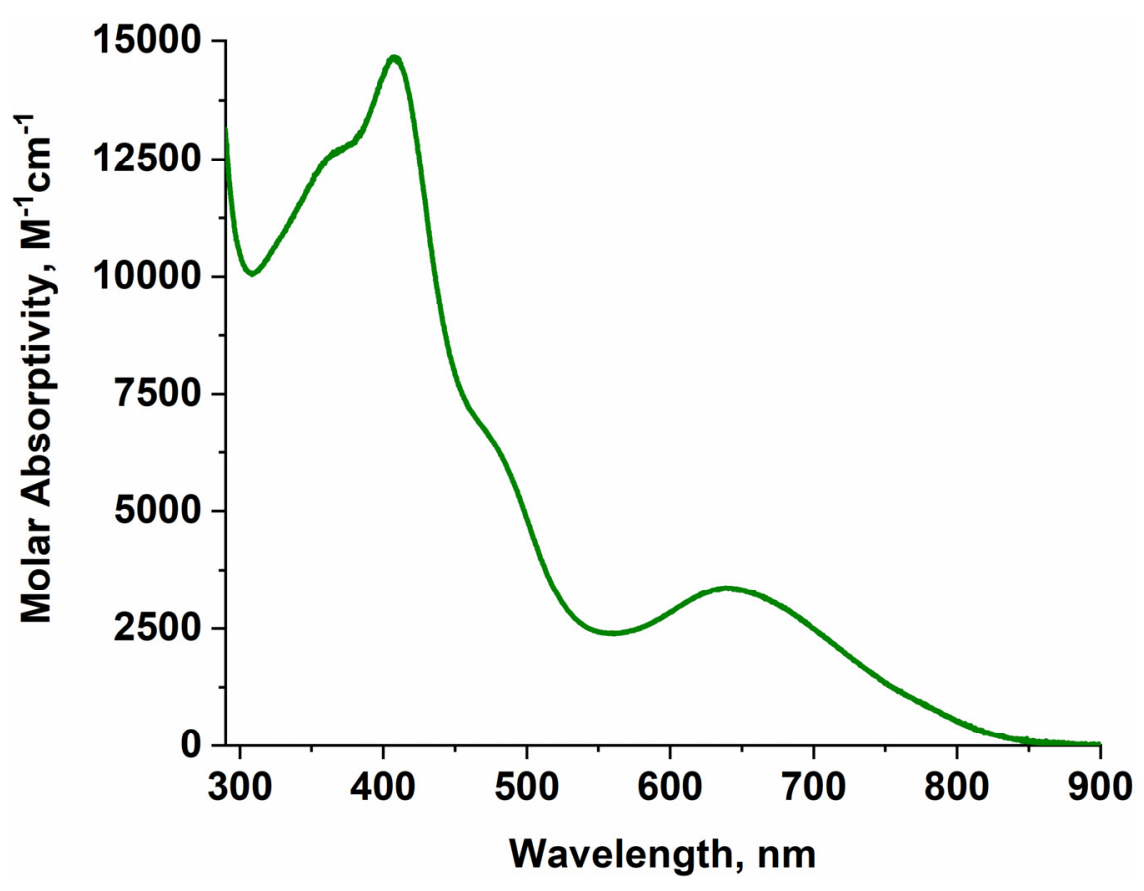

Figure S85 Electronic absorption spectrum of $\left({ }^{\mathrm{DIPP}} \mathrm{CNC}\right) \mathrm{Fe}\left(\mathrm{PMe}_{3}\right)\left(\mathrm{N}_{2}\right)$ recorded in benzene at ambient temperature.

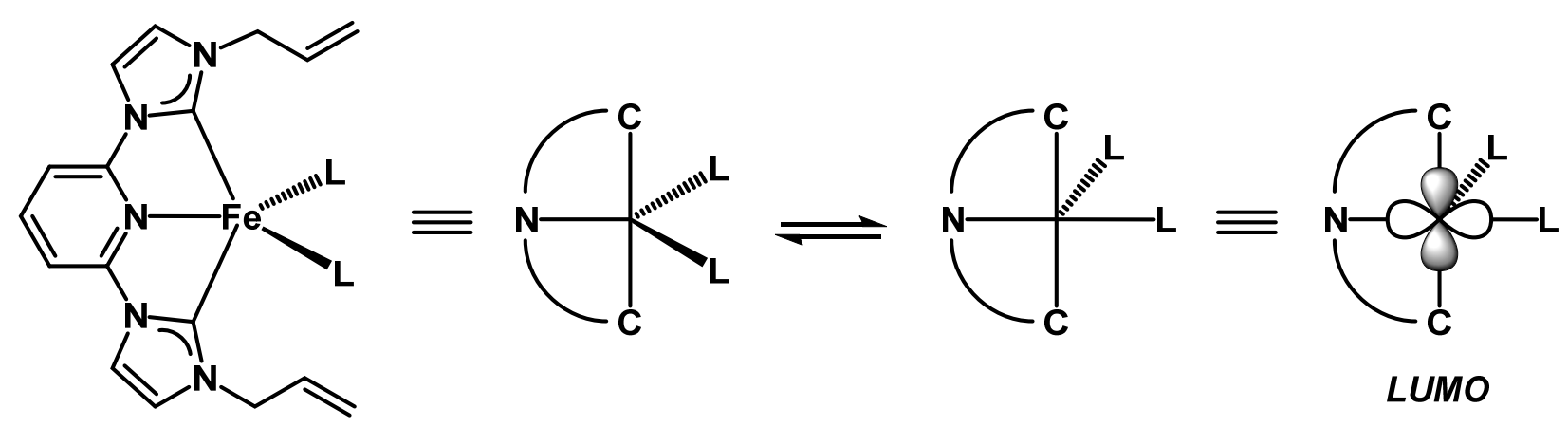

Scheme 1 Representation of LUMO in Fe(0) complexes. For MO diagram, see references. ${ }^{20}$ 
Compound: ( $\left.{ }^{\text {allyl }} \mathrm{CNC}\right) \mathrm{Fe}\left(\mathrm{PMe}_{3}\right)_{2}\left(3-\mathrm{PMe}_{3}\right)$

Local Name: JK1170

CCDC Number: 2084280

Table S1. Crystallographic details for ( $\left.{ }^{\text {allyl }} \mathrm{CNC}\right) \mathrm{Fe}\left(\mathrm{PMe}_{3}\right)_{2}\left(3-\mathrm{PMe}_{3}\right)$

\begin{tabular}{|c|c|}
\hline \multicolumn{2}{|l|}{ Crystal data } \\
\hline Chemical formula & $\mathrm{C}_{23} \mathrm{H}_{35} \mathrm{FeN}_{5} \mathrm{P}_{2}$ \\
\hline$M_{\mathrm{r}}$ & 499.35 \\
\hline Crystal system, space group & Monoclinic, $C c$ \\
\hline Temperature (K) & 85 \\
\hline$a, b, c(\AA)$ & $9.7150(4), 30.1880(8), 9.2926(3)$ \\
\hline$\beta\left(^{\circ}\right)$ & $114.537(4)$ \\
\hline$V\left(\AA^{3}\right)$ & $2479.19(16)$ \\
\hline$Z$ & 4 \\
\hline Radiation type & $\mathrm{Cu} K \alpha$ \\
\hline$\mu\left(\mathrm{mm}^{-1}\right)$ & 6.25 \\
\hline Crystal size (mm) & $0.09 \times 0.03 \times 0.02$ \\
\hline \multicolumn{2}{|l|}{ Data collection } \\
\hline Diffractometer & Abstract diffractometer \\
\hline Absorption correction & $\begin{array}{l}\text { Multi-scan CrysAlis PRO 1.171.38.41 (Rigaku Oxford Diffraction, } \\
\text { 2015) Empirical absorption correction using spherical harmonics, } \\
\text { implemented in SCALE3 ABSPACK scaling algorithm. }\end{array}$ \\
\hline$T_{\min }, T_{\max }$ & $0.829,1.000$ \\
\hline $\begin{array}{l}\text { No. of measured, independent and } \\
\text { observed }[I>2 s(I)] \text { reflections }\end{array}$ & $18895,4149,3642$ \\
\hline$R_{\text {int }}$ & 0.064 \\
\hline$(\sin \theta / \lambda)_{\max }\left(\AA^{-1}\right)$ & 0.607 \\
\hline \multicolumn{2}{|l|}{ Refinement } \\
\hline$R\left[F^{2}>2 \sigma\left(F^{2}\right)\right], w R\left(F^{2}\right), S$ & $0.055,0.142,1.03$ \\
\hline No. of reflections & 4149 \\
\hline No. of parameters & 315 \\
\hline No. of restraints & 77 \\
\hline $\mathrm{H}$-atom treatment & H-atom parameters constrained \\
\hline$\Delta \rho_{\max }, \Delta \rho_{\min }\left(\mathrm{e} \AA^{-3}\right)$ & $0.37,-0.60$ \\
\hline Absolute structure & Refined as an inversion twin. \\
\hline Absolute structure parameter & $-0.009(10)$ \\
\hline
\end{tabular}

Computer programs: CrysAlis PRO 1.171.38.41 (Rigaku OD, 2015), SHELXS97 (Sheldrick, 2008), SHELXL2014/7 (Sheldrick, 2014). 


\section{Refinement details:}

Refined as a 2-component inversion twin. An allyl substituent was modelled as disordered over two positions. Disordered fragments were refined to have similar geometries (SAME). The $\mathrm{U}^{\mathrm{ij}}$ components of atoms closer than $2.0 \AA$ A were restrained to be similar (SIMU). Subject to these conditions, the occupancies of the major and minor moieties are $0.592(18)$ to $0.408(18)$.

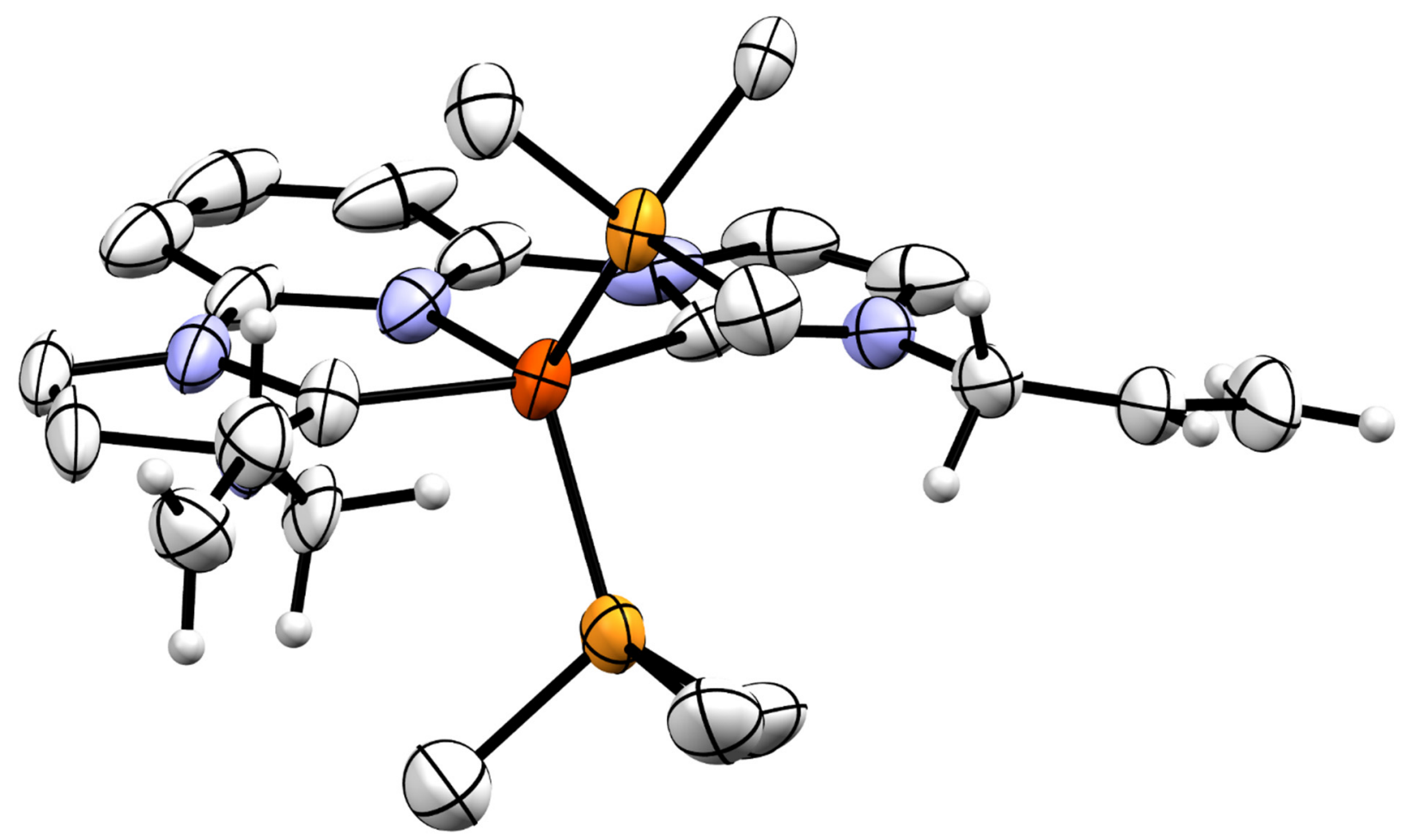

Figure S86 Molecular structure of (allyl $\mathrm{CNC}) \mathrm{Fe}\left(\mathrm{PMe}_{3}\right)_{2}\left(3-\mathrm{PMe}_{3}\right)$ displayed with $50 \%$ probability ellipsoids. All $\mathrm{H}$-atoms not attached to allyl fragments are omitted for clarity. The minor disordered moieties are omitted for clarity. 
Compound: (allyl $\mathrm{CNC}) \mathrm{Fe}\left(\mathrm{PEt}_{3}\right)_{2}\left(3-\mathrm{PEt}_{3}\right)$

Local Name: JK5268

CCDC Number: 2084289

Table S2. Crystallographic details for $\left({ }^{\text {allyl }} \mathrm{CNC}\right) \mathrm{Fe}\left(\mathrm{PEt}_{3}\right)_{2}\left(\right.$ (3-PEt $\left.{ }_{3}\right)$

\begin{tabular}{|c|c|}
\hline \multicolumn{2}{|l|}{ Crystal data } \\
\hline Chemical formula & $\mathrm{C}_{29} \mathrm{H}_{47} \mathrm{FeN}_{5} \mathrm{P}_{2}$ \\
\hline$M_{\mathrm{r}}$ & 583.50 \\
\hline Crystal system, space group & Monoclinic, $P 2_{1} / c$ \\
\hline Temperature (K) & 150 \\
\hline$a, b, c(\AA)$ & $31.6726(19), 9.6405$ (6), 20.7201 (13) \\
\hline$\beta\left(^{\circ}\right)$ & $105.5155(19)$ \\
\hline$V\left(\AA^{3}\right)$ & $6096.1(7)$ \\
\hline$Z$ & 8 \\
\hline Radiation type & Mo $K \alpha$ \\
\hline$\mu\left(\mathrm{mm}^{-1}\right)$ & 0.63 \\
\hline Crystal size $(\mathrm{mm})$ & $0.31 \times 0.27 \times 0.11$ \\
\hline \multicolumn{2}{|l|}{ Data collection } \\
\hline Diffractometer & Bruker AXS D8 Quest CMOS diffractometer \\
\hline Absorption correction & $\begin{array}{l}\text { Multi-scan SADABS 2016/2: Krause, L., Herbst-Irmer, R., Sheldrick } \\
\text { G.M. \& Stalke D., J. Appl. Cryst. } 48 \text { (2015) 3-10 }\end{array}$ \\
\hline$T_{\min }, T_{\max }$ & $0.561,0.746$ \\
\hline $\begin{array}{l}\text { No. of measured, independent and } \\
\text { observed }[I>2 s(I)] \text { reflections }\end{array}$ & $64095,15017,10750$ \\
\hline$R_{\text {int }}$ & 0.059 \\
\hline$(\sin \theta / \lambda)_{\max }\left(\AA^{-1}\right)$ & 0.668 \\
\hline \multicolumn{2}{|l|}{ Refinement } \\
\hline$R\left[F^{2}>2 \sigma\left(F^{2}\right)\right], w R\left(F^{2}\right), S$ & $0.052,0.124,1.07$ \\
\hline No. of reflections & 15017 \\
\hline No. of parameters & 699 \\
\hline No. of restraints & 37 \\
\hline $\mathrm{H}$-atom treatment & $\mathrm{H}$-atom parameters constrained \\
\hline$\Delta \rho_{\max }, \Delta \rho_{\min }\left(\mathrm{e} \AA^{-3}\right)$ & $0.52,-0.53$ \\
\hline
\end{tabular}

Computer programs: Apex3 v2018.7-2 (Bruker, 2018), SAINT V8.38A (Bruker, 2018), SHELXS97 (Sheldrick, 2008), SHELXL2018/3 (Sheldrick, 2015, 2018), SHELXLE Rev1030 (Hübschle et al., 2011). 


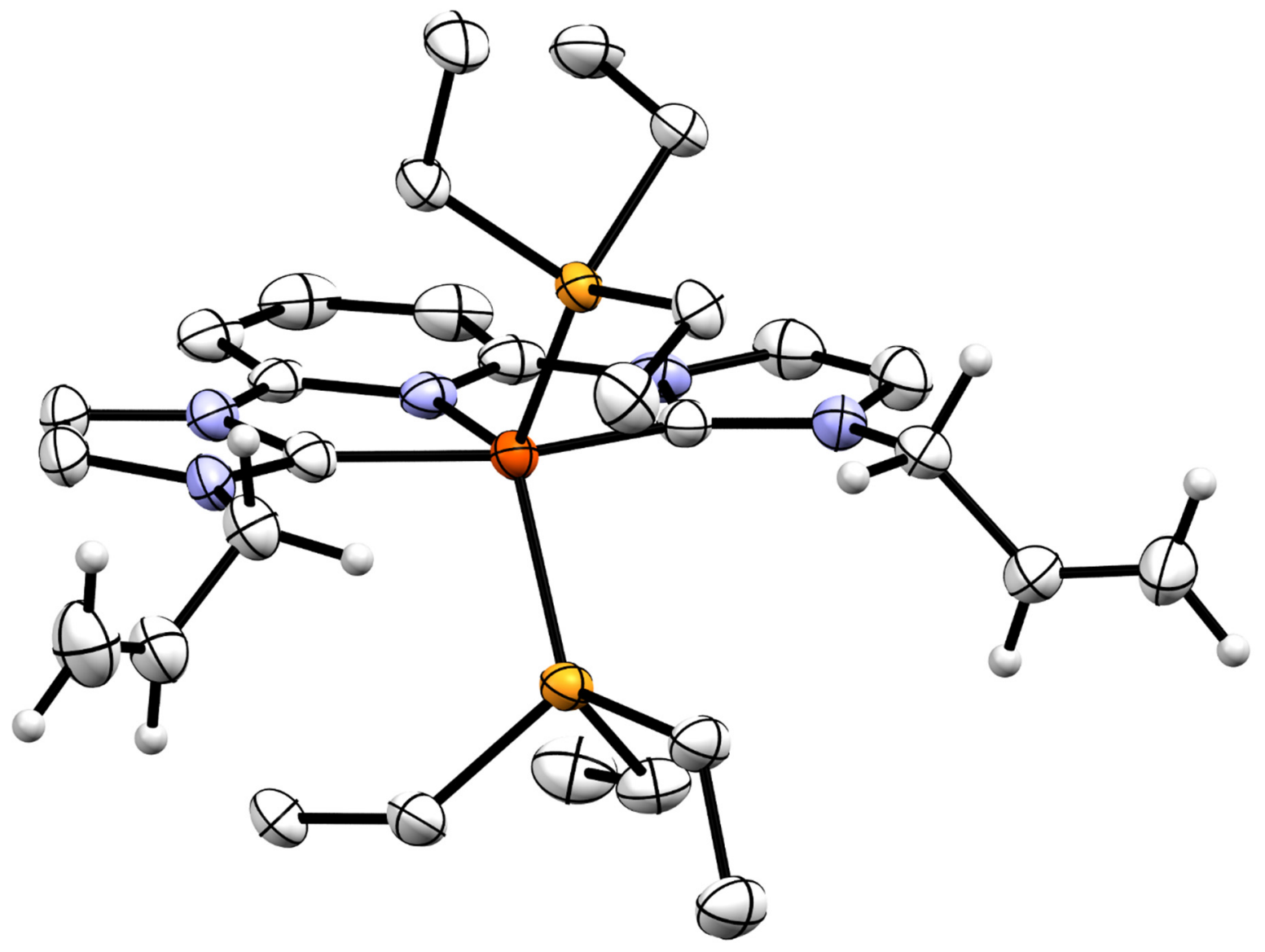

Figure S87 Molecular structure of $\left({ }^{\text {allyl }} \mathrm{CNC}\right) \mathrm{Fe}\left(\mathrm{PEt}_{3}\right)_{2}$ (3-PEt $\left.\mathrm{P}_{3}\right)$ displayed with $50 \%$ probability ellipsoids. All $\mathrm{H}$-atoms not attached to allyl fragments are omitted for clarity. 
Compound: ( $\left.{ }^{\text {allyl}} \mathrm{CNC}\right) \mathrm{Fe}($ depe) (3-depe)

Local Name: JK5269

CCDC Number: 2084291

Table S3. Crystallographic details for ( ${ }^{\text {allyl }} \mathrm{CNC}$ )Fe(depe) (3-depe)

\begin{tabular}{|c|c|}
\hline \multicolumn{2}{|l|}{ Crystal data } \\
\hline Chemical formula & $\mathrm{C}_{27} \mathrm{H}_{41} \mathrm{FeN}_{5} \mathrm{P}_{2}$ \\
\hline$M_{\mathrm{r}}$ & 553.44 \\
\hline Crystal system, space group & Orthorhombic, $P 2_{1} 2_{1} 2_{1}$ \\
\hline Temperature (K) & 150 \\
\hline$a, b, c(\AA)$ & 11.0800 (7), $19.1404(11), 38.350$ (2) \\
\hline$V\left(\AA^{3}\right)$ & $8133.0(9)$ \\
\hline$Z$ & 12 \\
\hline Radiation type & Mo $K \alpha$ \\
\hline$\mu\left(\mathrm{mm}^{-1}\right)$ & 0.70 \\
\hline Crystal size $(\mathrm{mm})$ & $0.55 \times 0.37 \times 0.21$ \\
\hline \multicolumn{2}{|l|}{ Data collection } \\
\hline Diffractometer & Bruker AXS D8 Quest CMOS diffractometer \\
\hline Absorption correction & $\begin{array}{l}\text { Multi-scan TWINABS 2012/1: Krause, L., Herbst-Irmer, R., Sheldrick } \\
\text { G.M. \& Stalke D. (2015). J. Appl. Cryst. } 48 \text { 3-10. }\end{array}$ \\
\hline$T_{\min }, T_{\max }$ & $0.670,0.747$ \\
\hline $\begin{array}{l}\text { No. of measured, independent and } \\
\text { observed }[I>2 s(I)] \text { reflections }\end{array}$ & $389927,64843,59513$ \\
\hline$R_{\text {int }}$ & 0.040 \\
\hline$(\sin \theta / \lambda)_{\max }\left(\AA^{-1}\right)$ & 0.771 \\
\hline \multicolumn{2}{|l|}{ Refinement } \\
\hline$R\left[F^{2}>2 \sigma\left(F^{2}\right)\right], w R\left(F^{2}\right), S$ & $0.049,0.193,1.04$ \\
\hline No. of reflections & 64843 \\
\hline No. of parameters & 960 \\
\hline $\mathrm{H}$-atom treatment & $\mathrm{H}$-atom parameters constrained \\
\hline$\Delta \rho_{\max }, \Delta \rho_{\min }\left(\mathrm{e} \AA^{-3}\right)$ & $1.80,-1.83$ \\
\hline Absolute structure & $\begin{array}{l}\text { Flack } x \text { determined using } 9950 \text { quotients }[(1+)-(I-)] /[(I+)+(I-)] \\
\text { (Parsons, Flack and Wagner, Acta Cryst. B69 (2013) 249-259). }\end{array}$ \\
\hline Absolute structure parameter & $-0.020(3)$ \\
\hline
\end{tabular}

Computer programs: Apex3 v2018.7-2 (Bruker, 2018), SAINT V8.38A (Bruker, 2018), SHELXS97 (Sheldrick,

2008), SHELXL2018/3 (Sheldrick, 2015, 2018), SHELXLE Rev1030 (Hübschle et al., 2011). 
The crystal under investigation was found to be a non-merohedric three component twin, emulating a double-the-volume approximate hexagonal structure with $a=22.1315, b=22.1412, c=38.3525$. The structure was originally solved in a monoclinic setting of this larger cell twinned by pseudomerohedry by two $120^{\circ}$ rotations, which established one of the $22 \AA$ axes to be only half that length. The three components were transformed into the smaller cell, and integrated as a non-merohedric twin with one third of reflections being overlapped between all three components, and two thirds of reflections being non-overlapped. The three components were integrated using Saint and corrected for absorption using twinabs, resulting in the following statistics:

113336 data (15592 unique) involve domain 1 only, mean l/sigma 12.9

113455 data (15579 unique) involve domain 2 only, mean I/sigma 12.4

113150 data (15577 unique) involve domain 3 only, mean I/sigma 11.3

87 data (82 unique) involve 2 domains, mean I/sigma 50.0

52231 data (17964 unique) involve 3 domains, mean I/sigma 43.1

34 data (34 unique) involve 4 domains, mean I/sigma 36.2

64 data (64 unique) involve 5 domains, mean I/sigma 34.4

2 data ( 2 unique) involve 6 domains, mean I/sigma 12.4

16 data (16 unique) involve 7 domains, mean I/sigma 42.5

The exact twin matrices identified by the integration program were found to be:

Twin Laws, Sample 1 of 1

Transforms h1.1(1)->h1.2(2)

$-0.50005-0.501310 .00001$

$1.49598-0.500050 .00001$

$-0.000020 .000071 .00000$

Transforms h1.1(1)->h1.3(3)

$0.49938-0.50153-0.00006$

$-1.49665-0.499380 .00004$

$-0.000560 .00027-1.00000$

Transforms h1.2(2)->h1.3(3)

$0.500570 .50114-0.00007$

$1.49547-0.500570 .00003$

$-0.00021-0.00046-1.00000$ 
The structure was solved using direct methods with only the non-overlapping reflections of component 1. The structure was refined using the hklf 5 routine with all reflections of all components (including the overlapping ones), resulting in BASF values of 0.3429(9) and 0.2962(9).

The $R_{\text {int }}$ value given is for all reflections and is based on agreement between observed single and composite intensities and those calculated from refined unique intensities and twin fractions (TWINABS (Sheldrick, 2012)).

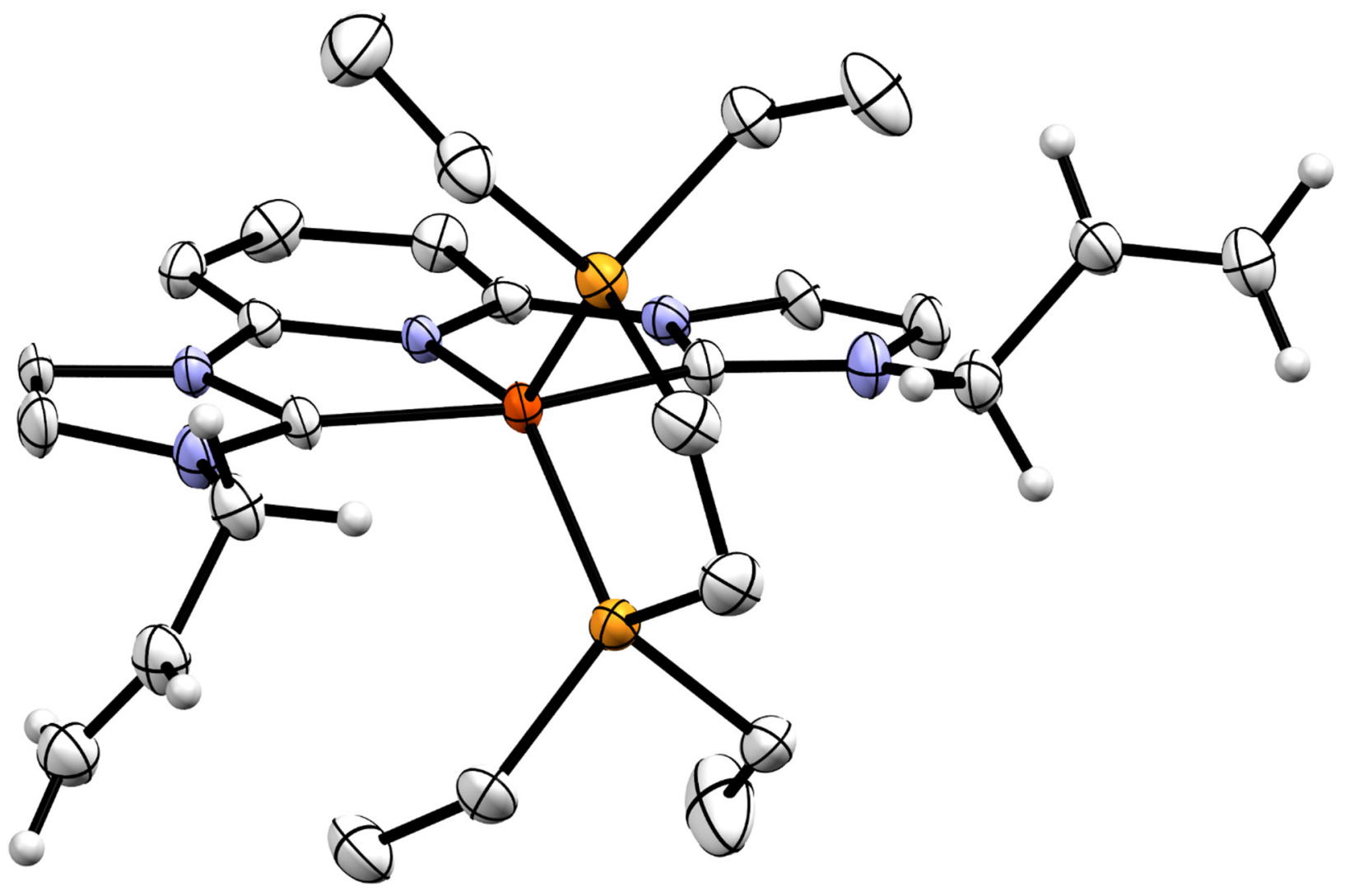

Figure S88 Molecular structure of (allyl CNC)Fe(depe) (3-depe) displayed with 50\% probability ellipsoids. All $\mathrm{H}$-atoms not attached to allyl fragments are omitted for clarity. Only one of the three independent molecules in the unit cell is displayed. 
Compound: [trans-( $\left.\left.{ }^{\text {allyl }} \mathrm{CNC}\right) \mathrm{FeBr}\left(\mathrm{PMe}_{3}\right)_{2}\right]\left[\mathrm{BPh}_{4}\right]$

Local Name: JK1172

CCDC Number: 2084284

Table S4. Crystallographic details for [trans-(allyl $\left.\mathrm{CNC}) \mathrm{FeBr}\left(\mathrm{PMe}_{3}\right)_{2}\right]\left[\mathrm{BPh}_{4}\right]$

\begin{tabular}{|c|c|}
\hline \multicolumn{2}{|l|}{ Crystal data } \\
\hline Chemical formula & $\mathrm{C}_{23} \mathrm{H}_{35} \mathrm{BrFeN}{ }_{5} \mathrm{P}_{2} \cdot \mathrm{C}_{24} \mathrm{H}_{20} \mathrm{~B} \cdot \mathrm{C}_{4} \mathrm{H}_{8} \mathrm{O}$ \\
\hline$M_{\mathrm{r}}$ & 970.57 \\
\hline Crystal system, space group & Triclinic, $P \overline{1}$ \\
\hline Temperature (K) & 85 \\
\hline$a, b, c(\AA)$ & $10.1812(2), 13.7593(2), 18.8328(3)$ \\
\hline$\alpha, \beta, \gamma\left({ }^{\circ}\right)$ & $108.954(1), 90.795(2), 102.513(1)$ \\
\hline$V\left(\AA^{3}\right)$ & $2425.74(7)$ \\
\hline$Z$ & 2 \\
\hline Radiation type & $\mathrm{Cu} K \alpha$ \\
\hline$\mu\left(\mathrm{mm}^{-1}\right)$ & 4.40 \\
\hline Crystal size $(\mathrm{mm})$ & $0.13 \times 0.12 \times 0.04$ \\
\hline \multicolumn{2}{|l|}{ Data collection } \\
\hline Diffractometer & Abstract diffractometer \\
\hline Absorption correction & $\begin{array}{l}\text { Multi-scan CrysAlis PRO 1.171.38.41 (Rigaku Oxford Diffraction, } \\
\text { 2015) Empirical absorption correction using spherical harmonics, } \\
\text { implemented in SCALE3 ABSPACK scaling algorithm. }\end{array}$ \\
\hline$T_{\min }, T_{\max }$ & $0.555,1.000$ \\
\hline $\begin{array}{l}\text { No. of measured, independent and } \\
\text { observed }[I>2 s(I)] \text { reflections }\end{array}$ & $36329,8784,8467$ \\
\hline$R_{\text {int }}$ & 0.033 \\
\hline$(\sin \theta / \lambda)_{\max }\left(\AA^{-1}\right)$ & 0.607 \\
\hline \multicolumn{2}{|l|}{ Refinement } \\
\hline$R\left[F^{2}>2 \sigma\left(F^{2}\right)\right], w R\left(F^{2}\right), S$ & $0.041,0.114,1.06$ \\
\hline No. of reflections & 8784 \\
\hline No. of parameters & 565 \\
\hline $\mathrm{H}$-atom treatment & $\mathrm{H}$-atom parameters constrained \\
\hline$\Delta \rho_{\max }, \Delta \rho_{\min }\left(\mathrm{e} \AA^{-3}\right)$ & $0.75,-1.10$ \\
\hline
\end{tabular}

Computer programs: CrysAlis PRO 1.171.38.41 (Rigaku OD, 2015), SHELXS97 (Sheldrick, 2008), SHELXL2014/7 (Sheldrick, 2014). 


\section{Comment on Structure}

In all instances where complexes of the type ( $\left.{ }^{\text {allyl }} \mathrm{CNC}\right) \mathrm{Fe}\left(\mathrm{PR}_{3}\right)_{2}(3)$ were successfully synthesized, an initial color change was noted (light orange to orange-red) upon addition of the phosphine to $\left[\left({ }^{\text {allyl }} \mathrm{CNC}\right) \mathrm{Fe}(\mathrm{MeCN})_{3}\right]\left[\mathrm{BPh}_{4}\right]_{2}$. This color change was noted for $\mathrm{PR}_{3}=\mathrm{PMe}_{3}, \mathrm{PEt}_{3}, \mathrm{PMe}_{2} \mathrm{Ph}$, and depe.

In all instances where a color change was not noted upon addition of the phosphine to $\left[\left({ }^{(\text {llylyl }} \mathrm{CNC}\right) \mathrm{Fe}(\mathrm{MeCN})_{3}\right]\left[\mathrm{BPh}_{4}\right]_{2}$, the desired complex of the type $\left({ }^{\text {allyl }} \mathrm{CNC}\right) \mathrm{Fe}\left(\mathrm{PR}_{3}\right)_{2}(3)$ was not able to be formed. This was observed when $\mathrm{PR}_{3}=\mathrm{PPh}_{3}$ and $\mathrm{PC} \mathrm{y}_{3}$.

We hypothesized that pre-coordination of the phosphine occurs to form species of the type [trans$\left.\left({ }^{\text {allyl }} \mathrm{CNC}\right) \mathrm{Fe}\left(\mathrm{PR}_{3}\right)_{2}(\mathrm{MeCN})\right]\left[\mathrm{BPh}_{4}\right]_{2}$. We were unable, however, to obtain structural evidence of this type of species. Therefore, co-crystallization attempts were undertaken. Single, X-ray quality crystals of [trans$\left.\left({ }^{\text {allyl }} \mathrm{CNC}\right) \mathrm{FeBr}\left(\mathrm{PMe}_{3}\right)_{2}\right]\left[\mathrm{BPh}_{4}\right]$ were obtained by diffusing $n$-pentane into a THF mixture of $\left[\left({ }^{\text {allyl }} \mathrm{CNC}\right) \mathrm{Fe}(\mathrm{MeCN})_{3}\right]\left[\mathrm{BPh}_{4}\right]_{2}, \mathrm{PMe}_{3}$ (2 equiv.), and $[\mathrm{Bu} 4 \mathrm{~N}][\mathrm{Br}]$ (1 equiv.) at room temperature.

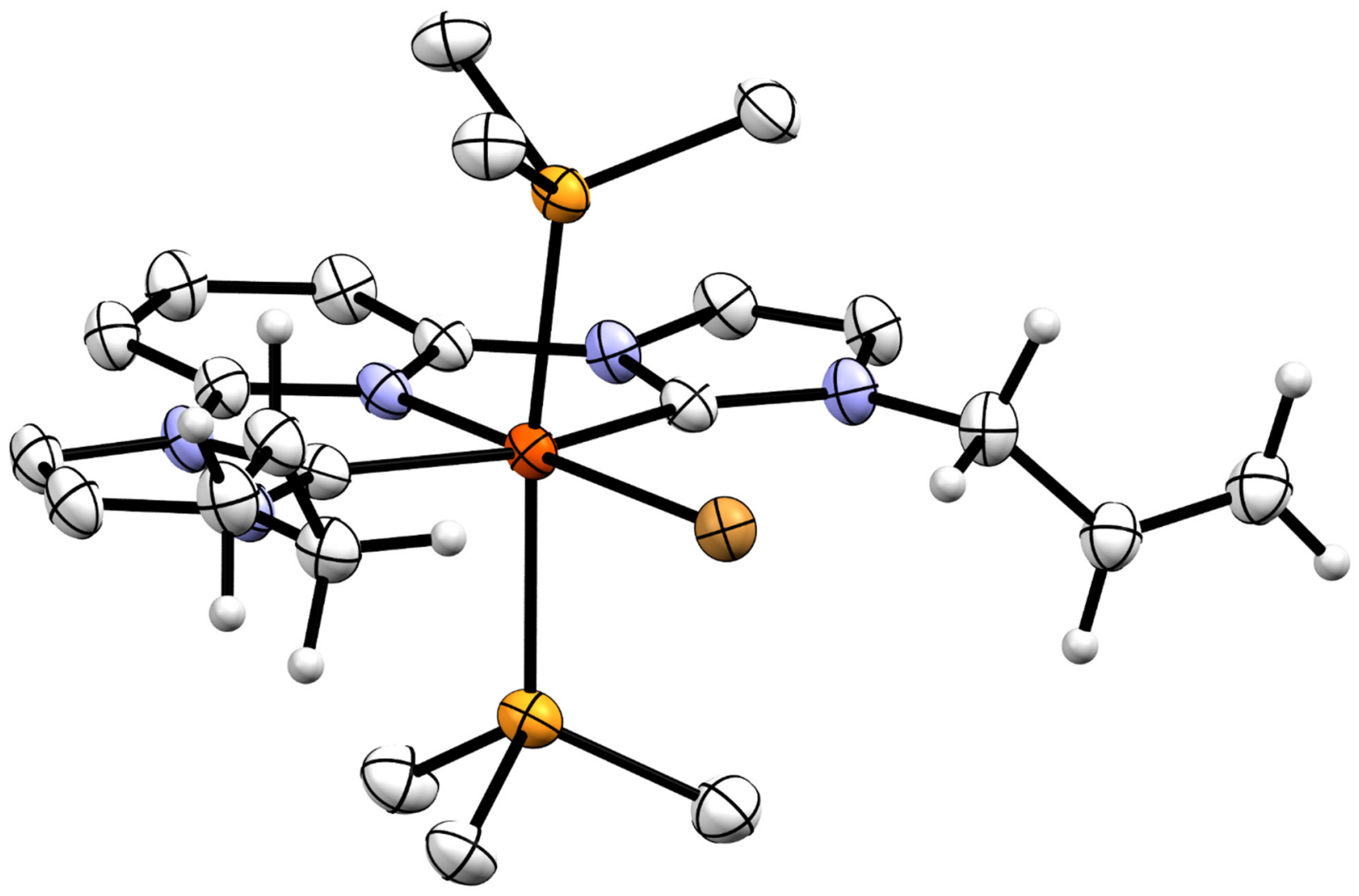

Figure S89 Molecular structure of the cationic portion of [trans-( $\left.\left.{ }^{\text {allyl }} \mathrm{CNC}\right) \mathrm{FeBr}\left(\mathrm{PMe}_{3}\right)_{2}\right]\left[\mathrm{BPh}_{4}\right]$ displayed with $50 \%$ probability ellipsoids. All $\mathrm{H}$-atoms are omitted except those of the allyl moiety. A co-crystallized tetrahydrofuran solvent molecule is omitted for clarity. Selected bond distances and angles: $\mathrm{Fe}-\mathrm{C}_{\text {carbene }}=$

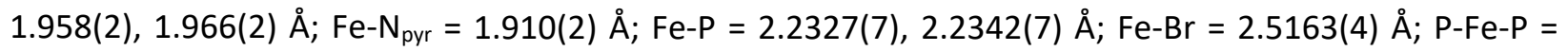
$170.91(3)^{\circ}$. 
Compound: (allyl $\mathrm{CNC}) \mathrm{Fe}(\mathrm{CNPh})_{2}(4-\mathrm{Ph})$

Local Name: JK2132

CCDC Number: 2084282

Table S5. Crystallographic details for ( $\left.{ }^{\text {allyl }} \mathrm{CNC}\right) \mathrm{Fe}(\mathrm{CNPh})_{2}$ (4-Ph)

\begin{tabular}{|c|c|}
\hline \multicolumn{2}{|l|}{ Crystal data } \\
\hline Chemical formula & $\mathrm{C}_{31} \mathrm{H}_{27} \mathrm{FeN}_{7}$ \\
\hline$M_{r}$ & 553.44 \\
\hline Crystal system, space group & Triclinic, $P \overline{1}$ \\
\hline Temperature (K) & 150 \\
\hline$a, b, c(\AA)$ & $9.7500(4), 10.9849(5), 13.2969(6)$ \\
\hline$\alpha, \beta, \gamma\left(^{\circ}\right)$ & 92.9123 (18), 94.8796 (18), 103.0317 (18) \\
\hline$V\left(\AA^{3}\right)$ & $1378.83(11)$ \\
\hline$Z$ & 2 \\
\hline Radiation type & $\mathrm{Cu} K \alpha$ \\
\hline$\mu\left(\mathrm{mm}^{-1}\right)$ & 4.64 \\
\hline Crystal size (mm) & $0.35 \times 0.18 \times 0.03$ \\
\hline \multicolumn{2}{|l|}{ Data collection } \\
\hline Diffractometer & Bruker AXS D8 Quest CMOS diffractometer \\
\hline Absorption correction & $\begin{array}{l}\text { Multi-scan SADABS 2016/2: Krause, L., Herbst-Irmer, R., Sheldrick } \\
\text { G.M. \& Stalke D., J. Appl. Cryst. } 48 \text { (2015) 3-10 }\end{array}$ \\
\hline$T_{\min }, T_{\max }$ & $0.040,0.178$ \\
\hline $\begin{array}{l}\text { No. of measured, independent and } \\
\text { observed }[I>2 s(I)] \text { reflections }\end{array}$ & $12874,5433,4658$ \\
\hline$R_{\text {int }}$ & 0.108 \\
\hline$(\sin \theta / \lambda)_{\max }\left(\AA^{-1}\right)$ & 0.638 \\
\hline \multicolumn{2}{|l|}{ Refinement } \\
\hline$R\left[F^{2}>2 \sigma\left(F^{2}\right)\right], w R\left(F^{2}\right), S$ & $0.084,0.233,1.08$ \\
\hline No. of reflections & 5433 \\
\hline No. of parameters & 372 \\
\hline No. of restraints & 50 \\
\hline $\mathrm{H}$-atom treatment & $\mathrm{H}$-atom parameters constrained \\
\hline$\Delta \rho_{\max }, \Delta \rho_{\min }\left(\mathrm{e} \AA^{-3}\right)$ & $0.60,-1.06$ \\
\hline
\end{tabular}

Computer programs: Apex3 v2016.9-0 (Bruker, 2016), SAINT V8.37A (Bruker, 2016), SHELXS97 (Sheldrick, 2008), SHELXL2017/1 (Sheldrick, 2017), SHELXLE Rev859 (Hübschle et al., 2011). 


\section{Refinement details:}

One allyl group was refined as disordered by rotation around the $\mathrm{C}-\mathrm{C}$ single bond. The two disordered moieties were restrained to have similar geometries. $U^{\mathrm{ij}}$ components of ADPs for disordered atoms closer to each other than $2.0 \AA$ were restrained to be similar. Subject to these conditions the occupancy ratio refined to $0.564(14)$ to $0.436(14)$.

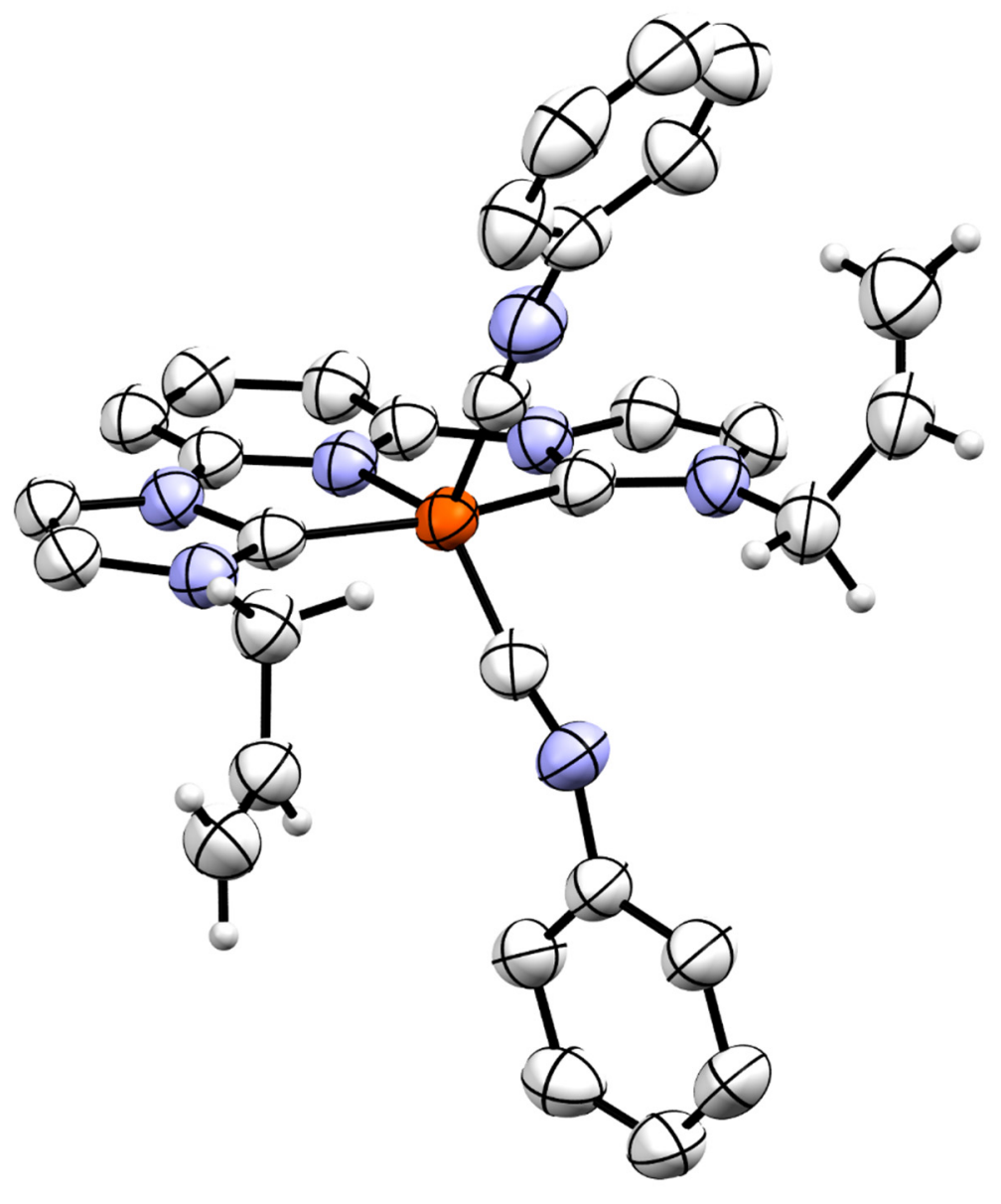

Figure $\mathbf{S 9 0}$ Molecular structure of (allyl $\mathrm{CNC}) \mathrm{Fe}(\mathrm{CNPh})_{2}$ (4-Ph) displayed with $50 \%$ probability ellipsoids. All $\mathrm{H}$-atoms are omitted except those attached to the allyl moieties. Disordered fragments are omitted for clarity. 
Compound: ( $\left.{ }^{\text {allyl }} \mathrm{CNC}\right) \mathrm{Fe}\left(\mathrm{CN}\left(2,4,6-{ }^{t} \mathrm{Bu}_{3} \mathrm{C}_{6} \mathrm{H}_{2}\right)\right)_{2}\left({ }^{4}-{ }^{t} \mathrm{Bu}_{3} \mathrm{C}_{6} \mathrm{H}_{2}\right)$

Local Name: JK269

CCDC Number: 2084286

Table S6. Crystallographic details for ( $\left.{ }^{\text {allylyl }} \mathrm{CNC}\right) \mathrm{Fe}\left(\mathrm{CN}\left(2,4,6-{ }^{\mathrm{t}} \mathrm{Bu}_{3} \mathrm{C}_{6} \mathrm{H}_{2}\right)\right)_{2}\left(4-{ }^{t} \mathrm{Bu}_{3} \mathrm{C}_{6} \mathrm{H}_{2}\right)$

\begin{tabular}{|c|c|}
\hline \multicolumn{2}{|l|}{ Crystal data } \\
\hline Chemical formula & $\mathrm{C}_{55} \mathrm{H}_{75} \mathrm{FeN}_{7}$ \\
\hline$M_{\mathrm{r}}$ & 890.07 \\
\hline Crystal system, space group & Triclinic, $P \overline{1}$ \\
\hline Temperature (K) & 150 \\
\hline$a, b, c(\AA)$ & $10.779(4), 26.526(7), 37.943(10)$ \\
\hline$\alpha, \beta, \gamma\left({ }^{\circ}\right)$ & 104.235 (12), 95.135 (17), 94.278 (13) \\
\hline$V\left(\AA^{3}\right)$ & $10421(5)$ \\
\hline$Z$ & 8 \\
\hline Radiation type & $\mathrm{Cu} K \alpha$ \\
\hline$\mu\left(\mathrm{mm}^{-1}\right)$ & 2.63 \\
\hline Crystal size (mm) & $0.35 \times 0.15 \times 0.06$ \\
\hline \multicolumn{2}{|l|}{ Data collection } \\
\hline Diffractometer & Bruker AXS D8 Quest CMOS diffractometer \\
\hline Absorption correction & $\begin{array}{l}\text { Multi-scan SADABS 2016/2: Krause, L., Herbst-Irmer, R. } \\
\text { Sheldrick G.M. \& Stalke D., J. Appl. Cryst. } 48 \text { (2015) 3-10 }\end{array}$ \\
\hline$T_{\min }, T_{\max }$ & $0.459,0.754$ \\
\hline $\begin{array}{l}\text { No. of measured, independent and } \\
\text { observed }[I>2 \mathrm{~s}(I)] \text { reflections }\end{array}$ & $113534,39283,24622$ \\
\hline$R_{\text {int }}$ & 0.083 \\
\hline$(\sin \theta / \lambda)_{\max }\left(\AA^{-1}\right)$ & 0.622 \\
\hline \multicolumn{2}{|l|}{ Refinement } \\
\hline$R\left[F^{2}>2 \sigma\left(F^{2}\right)\right], w R\left(F^{2}\right), S$ & $0.062,0.173,1.02$ \\
\hline No. of reflections & 39283 \\
\hline No. of parameters & 3235 \\
\hline No. of restraints & 5558 \\
\hline $\mathrm{H}$-atom treatment & $\mathrm{H}$-atom parameters constrained \\
\hline$\Delta \rho_{\max }, \Delta \rho_{\min }\left(\mathrm{e} \AA^{-3}\right)$ & $1.27,-0.59$ \\
\hline
\end{tabular}

Computer programs: Apex3 v2016.9-0 (Bruker, 2016), SAINT V8.37A (Bruker, 2016), SHELXS97 (Sheldrick, 2008), SHELXL2016/6 (Sheldrick, 2015, 2016), SHELXLE Rev714 (Hübschle et al., 2011). 


\section{Refinement details:}

All four crystallographically independent molecules exhibit substantial disorder. For all four, the chelating ligand is disordered by a swinging motion around the iron center. For molecules $B$ and $C$, the Fe center was included in the disorder. For molecules $B$ and $C$, there is also rotational disorder of one of the tertbutyl groups. All eight chelating ligand moieties were restrained to have similar geometries. The disordered tert-butyl groups were restrained to have a geometry similar to that of another not disordered tert-butyl group. $\mathrm{U}^{\mathrm{ij}}$ components of ADPs of all disordered atoms were restrained to be similar for atoms closer to each other than $2.0 \AA$. Subject to these conditions the occupancy ratios refined for the chelating ligand to $0.835(5)$ to $0.165(5)$ for molecules $A$ and $E$, to $0.805(3)$ to $0.195(3)$ for molecules $B$ and $F$, to $0.616(5)$ to $0.384(5)$ for molecules $C$ and G, and to $0.815(6)$ to $0.185(6)$ for molecules $D$ and $H$. The tertbutyl ratios refined to $0.586(5)$ to $0.414(5)$ for molecules $B$ and $F$, to $0.873(5)$ to $0.127(5)$ for molecules $C$ and $\mathrm{G}$.

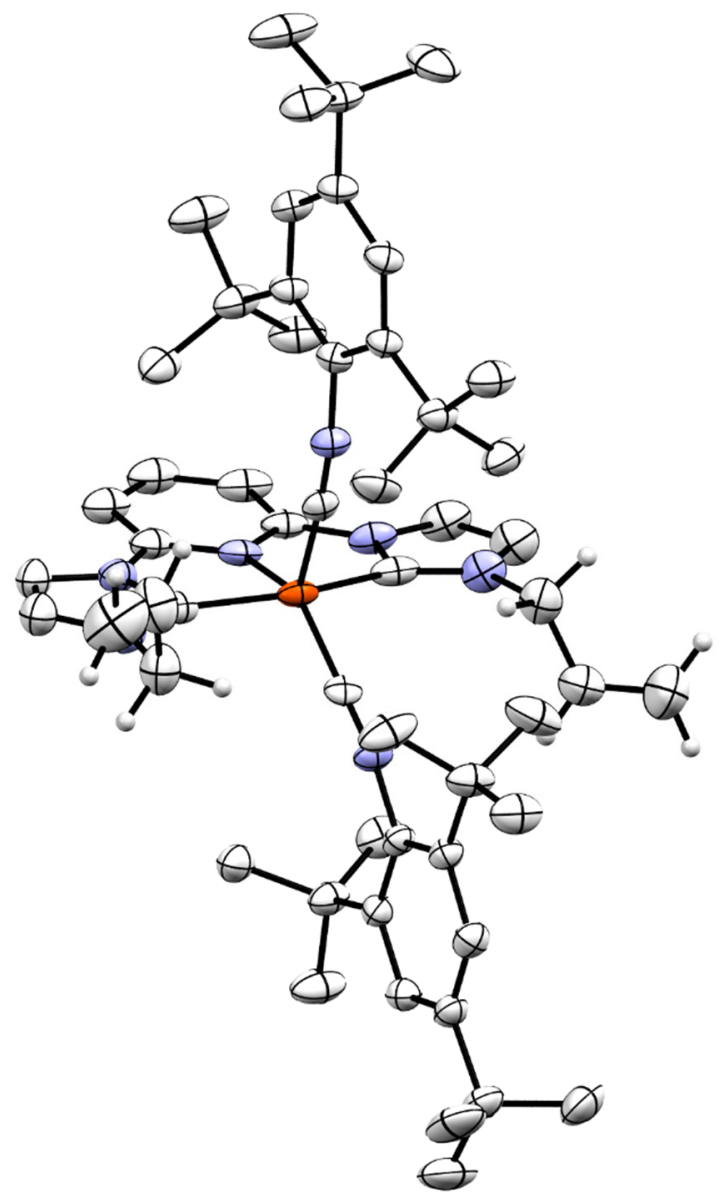

Figure S91 Molecular structure of one of the four independent molecules of (allyl $\mathrm{CNC}) \mathrm{Fe}(\mathrm{CN}(2,4,6$ $\left.\left.{ }^{t} \mathrm{Bu}_{3} \mathrm{C}_{6} \mathrm{H}_{2}\right)\right)_{2}\left({ }_{4-}{ }^{t} \mathrm{Bu}_{3} \mathrm{C}_{6} \mathrm{H}_{2}\right)$ displayed with $50 \%$ probability ellipsoids. All $\mathrm{H}$-atoms are omitted except those of the allyl moiety. Disordered fragments are omitted for clarity. 
Compound: ( $\left.{ }^{\text {allyl}} \mathrm{CNC}\right) \mathrm{Fe}\left(\mathrm{CN}^{t} \mathrm{Bu}\right)_{2}\left(4-{ }^{t} \mathrm{Bu}\right)$

Local Name: JK5292

CCDC Number: 2084290

Table S7. Crystallographic details for $\left({ }^{\text {allyl }} \mathrm{CNC}\right) \mathrm{Fe}\left(\mathrm{CN}^{t} \mathrm{Bu}\right)_{2}\left(4^{-}{ }^{t} \mathrm{Bu}\right)$

\begin{tabular}{|c|c|}
\hline \multicolumn{2}{|l|}{ Crystal data } \\
\hline Chemical formula & $\mathrm{C}_{27} \mathrm{H}_{35} \mathrm{FeN}_{7}$ \\
\hline$M_{\mathrm{r}}$ & 513.47 \\
\hline Crystal system, space group & Monoclinic, $C 2 / c$ \\
\hline Temperature (K) & 150 \\
\hline$a, b, c(\AA)$ & $19.0476(8), 13.4960(6), 11.3325(5)$ \\
\hline$\beta\left(^{\circ}\right)$ & $118.592(2)$ \\
\hline$V\left(\AA^{3}\right)$ & $2557.9(2)$ \\
\hline$Z$ & 4 \\
\hline Radiation type & $\mathrm{Cu} K \alpha$ \\
\hline$\mu\left(\mathrm{mm}^{-1}\right)$ & 4.95 \\
\hline Crystal size (mm) & $0.41 \times 0.23 \times 0.18$ \\
\hline \multicolumn{2}{|l|}{ Data collection } \\
\hline Diffractometer & $\begin{array}{l}\text { Bruker AXS D8 Quest diffractometer with PhotonIII_C14 charge- } \\
\text { integrating pixel array detector (CPAD) }\end{array}$ \\
\hline Absorption correction & $\begin{array}{l}\text { Multi-scan SADABS 2016/2: Krause, L., Herbst-Irmer, R., Sheldrick } \\
\text { G.M. \& Stalke D., J. Appl. Cryst. } 48 \text { (2015) 3-10 }\end{array}$ \\
\hline$T_{\min }, T_{\max }$ & $0.435,0.754$ \\
\hline $\begin{array}{l}\text { No. of measured, independent and } \\
\text { observed }[I>2 s(I)] \text { reflections }\end{array}$ & $22487,2754,2697$ \\
\hline$R_{\text {int }}$ & 0.040 \\
\hline$(\sin \theta / \lambda)_{\max }\left(\AA^{-1}\right)$ & 0.638 \\
\hline \multicolumn{2}{|l|}{ Refinement } \\
\hline$R\left[F^{2}>2 \sigma\left(F^{2}\right)\right], w R\left(F^{2}\right), S$ & $0.034,0.096,1.21$ \\
\hline No. of reflections & 2754 \\
\hline No. of parameters & 163 \\
\hline $\mathrm{H}$-atom treatment & $\mathrm{H}$-atom parameters constrained \\
\hline$\Delta \rho_{\max }, \Delta \rho_{\min }\left(\mathrm{e} \AA^{-3}\right)$ & $0.30,-0.41$ \\
\hline
\end{tabular}

Computer programs: Apex3 v2019.1-0 (Bruker, 2019), SAINT V8.40A (Bruker, 2019), SHELXS97 (Sheldrick,

2008), SHELXL2018/3 (Sheldrick, 2015, 2018), SHELXLE Rev1117 (Hübschle et al., 2011). 


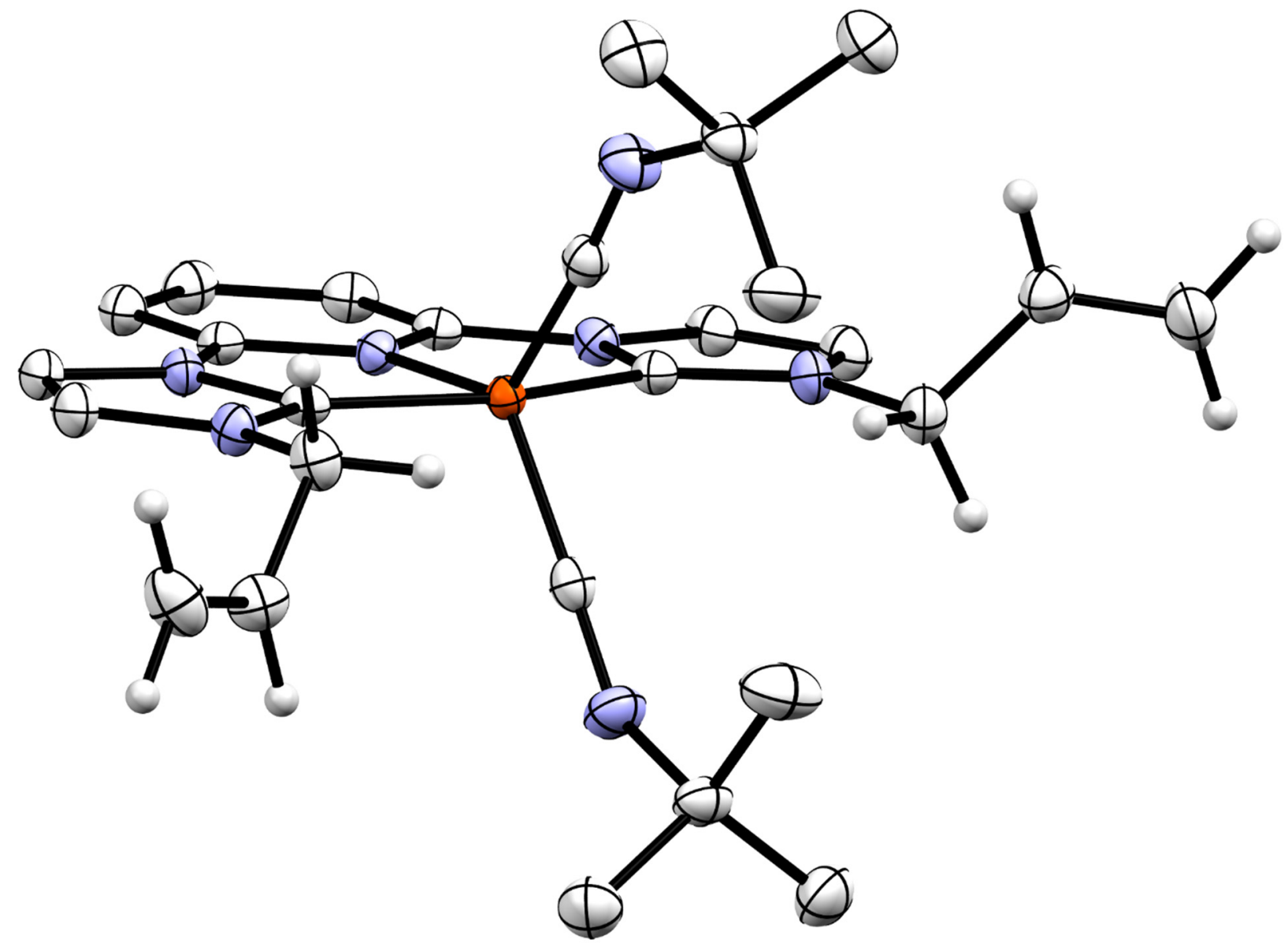

Figure S92 Molecular structure of $\left({ }^{\text {allyl }} \mathrm{CNC}\right) \mathrm{Fe}\left(\mathrm{CN}^{t} \mathrm{Bu}\right)_{2}\left(4^{-}{ }^{t} \mathrm{Bu}\right)$ displayed with $50 \%$ probability ellipsoids. All $\mathrm{H}$-atoms are omitted except those attached to the allyl moieties. 
Compound: $\left[\left({ }^{\mathrm{BBN}} \mathrm{CNC}\right) \mathrm{Fe}\left(\mathrm{PMe}_{2} \mathrm{Ph}\right)_{2}(\mathrm{H})(\mathrm{OH})\right]_{2}$

Local Name: JK5263

CCDC Number: 2084288

Table S8. Crystallographic details for $\left[\left({ }^{\mathrm{BBN}} \mathrm{CNC}\right) \mathrm{Fe}\left(\mathrm{PMe}{ }_{2} \mathrm{Ph}\right)_{2}(\mathrm{H})(\mathrm{OH})\right]_{2}$

\begin{tabular}{|c|c|}
\hline \multicolumn{2}{|l|}{ Crystal data } \\
\hline Chemical formula & $\mathrm{C}_{98} \mathrm{H}_{142} \mathrm{~B}_{4} \mathrm{Fe}_{2} \mathrm{~N}_{10} \mathrm{O}_{2} \mathrm{P}_{4} \cdot 6\left(\mathrm{C}_{6} \mathrm{H}_{6}\right)$ \\
\hline$M_{\mathrm{r}}$ & 2239.67 \\
\hline Crystal system, space group & Monoclinic, $P 2_{1} / c$ \\
\hline Temperature (K) & 150 \\
\hline$a, b, c(\AA)$ & 17.3035 (8), 10.4624 (5), 34.0075 (14) \\
\hline$\beta\left(^{\circ}\right)$ & $90.481(3)$ \\
\hline$V\left(\AA^{3}\right)$ & $6156.4(5)$ \\
\hline$Z$ & 2 \\
\hline Radiation type & $\mathrm{Cu} K \alpha$ \\
\hline$\mu\left(\mathrm{mm}^{-1}\right)$ & 2.80 \\
\hline Crystal size (mm) & $0.21 \times 0.04 \times 0.04$ \\
\hline \multicolumn{2}{|l|}{ Data collection } \\
\hline Diffractometer & $\begin{array}{l}\text { Bruker AXS D8 Quest CMOS diffractometer with Photonll charge- } \\
\text { integrating pixel array detector (CPAD) }\end{array}$ \\
\hline Absorption correction & $\begin{array}{l}\text { Multi-scan SADABS 2016/2: Krause, L., Herbst-Irmer, R., Sheldrick } \\
\text { G.M. \& Stalke D., J. Appl. Cryst. } 48 \text { (2015) 3-10 }\end{array}$ \\
\hline$T_{\min }, T_{\max }$ & $0.471,0.754$ \\
\hline $\begin{array}{l}\text { No. of measured, independent and } \\
\text { observed }[I>2 \mathrm{~s}(I)] \text { reflections }\end{array}$ & $39976,11875,10127$ \\
\hline$R_{\text {int }}$ & 0.053 \\
\hline$(\sin \theta / \lambda)_{\max }\left(\AA^{-1}\right)$ & 0.620 \\
\hline \multicolumn{2}{|l|}{ Refinement } \\
\hline$R\left[F^{2}>2 \sigma\left(F^{2}\right)\right], w R\left(F^{2}\right), S$ & $0.049,0.131,1.06$ \\
\hline No. of reflections & 11875 \\
\hline No. of parameters & 852 \\
\hline No. of restraints & 360 \\
\hline $\mathrm{H}$-atom treatment & $\begin{array}{l}\mathrm{H} \text { atoms treated by a mixture of independent and constrained } \\
\text { refinement }\end{array}$ \\
\hline$\Delta \rho_{\max }, \Delta \rho_{\min }\left(\mathrm{e} \AA^{-3}\right)$ & $0.69,-0.27$ \\
\hline
\end{tabular}

Computer programs: Apex3 v2018.7-2 (Bruker, 2018), SAINT V8.38A (Bruker, 2018), SHELXS97 (Sheldrick, 2008), SHELXL2018/3 (Sheldrick, 2015, 2018), SHELXLE Rev1030 (Hübschle et al., 2011). 


\section{Refinement details:}

Hydroxyl and hydride $\mathrm{H}$ atom positions and thermal parameters were freely refined.

Two benzene solvate molecules were refined as disordered by slight rotation. Disordered benzene rings were constrained to resemble ideal hexagons with C-C distances of $1.39 \AA$. U $U^{\mathrm{ij}}$ components of ADPs for disordered atoms closer to each other than $2.0 \AA \AA$ were restrained to be similar. Subject to these conditions the occupancy ratio refined to $0.45(3)$ to $0.55(3)$ and $0.484(9)$ to $0.516(9)$.

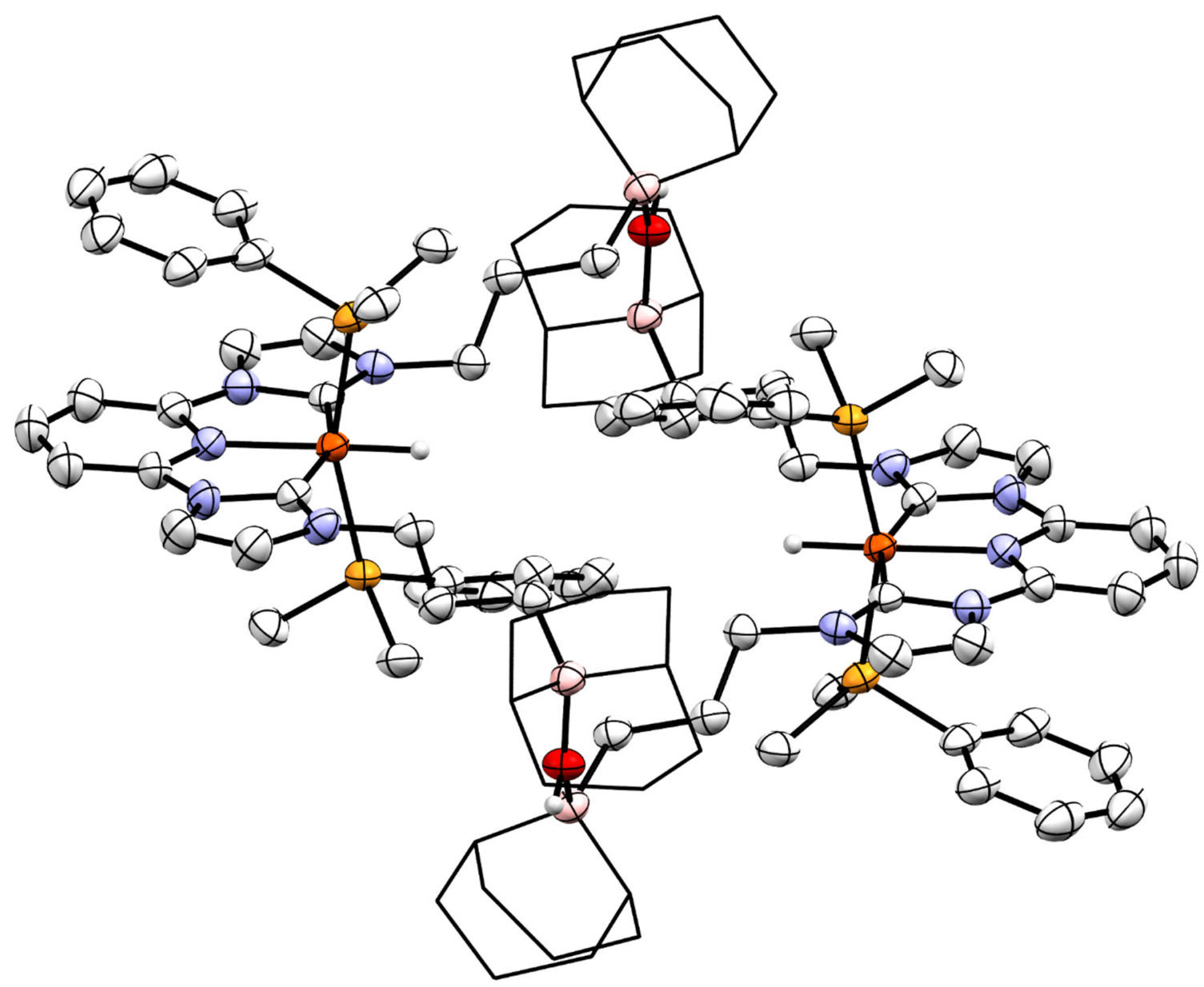

Figure S93 Molecular structure of $\left[\left({ }^{\mathrm{BBN}} \mathrm{CNC}\right) \mathrm{Fe}\left(\mathrm{PMe} \mathrm{P}_{2} \mathrm{Ph}\right)_{2}(\mathrm{H})(\mathrm{OH})\right]_{2}$ displayed with $50 \%$ probability ellipsoids. All $\mathrm{H}$-atoms are omitted except the hydride and hydroxyl. The 9-BBN moieties are displayed in wireframe for improved clarity. Co-crystallized benzene solvent molecules are omitted for clarity. 
Compound: ( $\left.{ }^{\mathrm{BBN}} \mathrm{CNC}\right) \mathrm{Fe}\left(\mathrm{CN}\left(2,4,6-{ }^{\mathrm{t}} \mathrm{Bu}_{3} \mathrm{C}_{6} \mathrm{H}_{2}\right)\right)_{2}\left(5^{-}{ }^{\mathrm{B}} \mathrm{Bu}_{3} \mathrm{C}_{6} \mathrm{H}_{2}\right)$

Local Name: JK297

CCDC Number: 2084285

Table S9. Crystallographic details for $\left({ }^{\mathrm{BBN}} \mathrm{CNC}\right) \mathrm{Fe}\left(\mathrm{CN}\left(2,4,6-{ }^{\mathrm{t}} \mathrm{Bu}_{3} \mathrm{C}_{6} \mathrm{H}_{2}\right)\right)_{2}\left(5-{ }^{t} \mathrm{Bu}_{3} \mathrm{C}_{6} \mathrm{H}_{2}\right)$

\begin{tabular}{|c|c|}
\hline \multicolumn{2}{|l|}{ Crystal data } \\
\hline Chemical formula & $2\left(\mathrm{C}_{71} \mathrm{H}_{105} \mathrm{~B}_{2} \mathrm{FeN}_{7}\right) \cdot 3\left(\mathrm{C}_{5} \mathrm{H}_{12}\right)$ \\
\hline$M_{\mathrm{r}}$ & 2484.63 \\
\hline Crystal system, space group & Monoclinic, $P 2_{1} / C$ \\
\hline Temperature (K) & 150 \\
\hline$a, b, c(\AA)$ & 18.0901 (8), $39.2327(17), 10.6770$ (5) \\
\hline$\beta\left(^{\circ}\right)$ & $90.3966(15)$ \\
\hline$V\left(\AA^{3}\right)$ & $7577.5(6)$ \\
\hline$Z$ & 2 \\
\hline Radiation type & 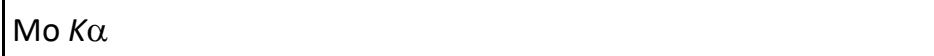 \\
\hline$\mu\left(\mathrm{mm}^{-1}\right)$ & 0.24 \\
\hline Crystal size $(\mathrm{mm})$ & $0.29 \times 0.21 \times 0.13$ \\
\hline \multicolumn{2}{|l|}{ Data collection } \\
\hline Diffractometer & Bruker AXS D8 Quest CMOS diffractometer \\
\hline Absorption correction & $\begin{array}{l}\text { Multi-scan SADABS 2016/2: Krause, L., Herbst-Irmer, R., Sheldrick } \\
\text { G.M. \& Stalke D., J. Appl. Cryst. } 48 \text { (2015) 3-10 }\end{array}$ \\
\hline$T_{\min }, T_{\max }$ & $0.700,0.746$ \\
\hline $\begin{array}{l}\text { No. of measured, independent and } \\
\text { observed }[I>2 \mathrm{~s}(I)] \text { reflections }\end{array}$ & $81110,22903,19388$ \\
\hline$R_{\text {int }}$ & 0.024 \\
\hline$(\sin \theta / \lambda)_{\max }\left(\AA^{-1}\right)$ & 0.716 \\
\hline \multicolumn{2}{|l|}{ Refinement } \\
\hline$R\left[F^{2}>2 \sigma\left(F^{2}\right)\right], w R\left(F^{2}\right), S$ & $0.041,0.108,1.02$ \\
\hline No. of reflections & 22903 \\
\hline No. of parameters & 963 \\
\hline No. of restraints & 465 \\
\hline $\mathrm{H}$-atom treatment & H-atom parameters constrained \\
\hline$\Delta \rho_{\max }, \Delta \rho_{\min }\left(\mathrm{e} \AA^{-3}\right)$ & $0.41,-0.40$ \\
\hline
\end{tabular}

Computer programs: Apex3 v2016.9-0 (Bruker, 2016), SAINT V8.37A (Bruker, 2016), SHELXS97 (Sheldrick, 2008), SHELXL2016/6 (Sheldrick, 2015, 2016), SHELXLE Rev714 (Hübschle et al., 2011). 


\section{Refinement details:}

One of the borabicyclononane units is disordered by a pseudo-mirror operation with the boron atom in common to both units. The geometries of the two moieties were restrained to be similar to each other and $\mathrm{U}^{\mathrm{ij}}$ components of ADPs were restrained to be similar for disordered atoms closer to each other than $2.0 \AA$. Subject to these conditions the occupancy ratio refined to $0.9228(16)$ to $0.0772(16)$.

Two pentane molecules are disordered. One around an inversion center, and another over two alternative orientations in a general position. All three unique moieties were restrained to have similar geometries, and $\mathrm{U}^{\mathrm{ij}}$ components of ADPs were restrained to be similar for disordered atoms closer to each other than $2.0 \AA$. Subject to these conditions the occupancy ratio of the pentane molecule in the general position refined to $0.831(4)$ to $0.169(4)$.

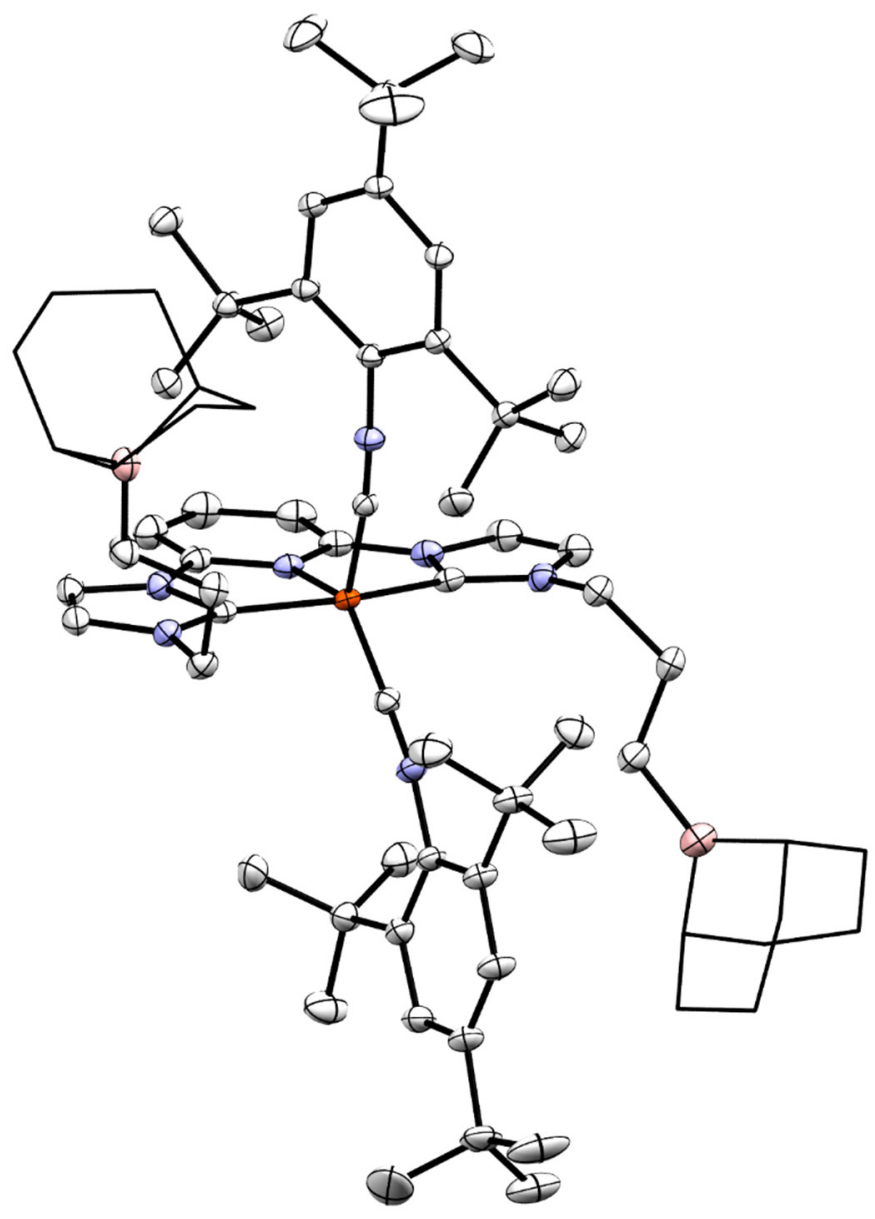

Figure S94 Molecular structure of $\left({ }^{\mathrm{BBN}} \mathrm{CNC}\right) \mathrm{Fe}\left(\mathrm{CN}\left(2,4,6-{ }^{\mathrm{t}} \mathrm{Bu}_{3} \mathrm{C}_{6} \mathrm{H}_{2}\right)\right)_{2} \quad\left(5-{ }^{\mathrm{t}} \mathrm{Bu}_{3} \mathrm{C}_{6} \mathrm{H}_{2}\right)$ displayed with $50 \%$ probability ellipsoids. All $\mathrm{H}$-atoms are omitted and the -BBN fragments are displayed in wireframe for improved clarity. Disordered fragments and co-crystallized $n$-pentane molecules are omitted for clarity. 
Compound: $\left({ }^{\mathrm{BPin}} \mathrm{CNC}\right) \mathrm{Fe}\left(\mathrm{CN}\left(2,4,6-{ }^{t} \mathrm{Bu}_{3} \mathrm{C}_{6} \mathrm{H}_{2}\right)\right)_{2}\left(6-\mathrm{CN}^{t} \mathrm{Bu}_{3} \mathrm{C}_{6} \mathrm{H}_{2}\right)$

Local Name: JK215B

CCDC Number: 2084283

Table S10. Crystallographic details for $\left({ }^{\mathrm{BPin}} \mathrm{CNC}\right) \mathrm{Fe}\left(\mathrm{CN}\left(2,4,6-{ }^{\mathrm{t}} \mathrm{Bu}_{3} \mathrm{C}_{6} \mathrm{H}_{2}\right)\right)_{2}\left(6-\mathrm{CN}^{t} \mathrm{Bu}_{3} \mathrm{C}_{6} \mathrm{H}_{2}\right)$

\begin{tabular}{|c|c|}
\hline \multicolumn{2}{|l|}{ Crystal data } \\
\hline Chemical formula & $\mathrm{C}_{67} \mathrm{H}_{101} \mathrm{~B}_{2} \mathrm{FeN}_{7} \mathrm{O}_{4}$ \\
\hline$M r$ & 1146.01 \\
\hline Crystal system, space group & Monoclinic, $P 2_{1} / c$ \\
\hline Temperature (K) & 150 \\
\hline$a, b, c(\AA)$ & $13.9544(6), 14.6358(7), 33.4179(16)$ \\
\hline$\beta\left(\left(^{\circ}\right)\right.$ & $98.577(2)$ \\
\hline$V\left(\AA^{3}\right)$ & $6748.7(5)$ \\
\hline$Z$ & 4 \\
\hline Radiation type & Mo $K \alpha$ \\
\hline$\mu\left(\mathrm{mm}^{-1}\right)$ & 0.27 \\
\hline Crystal size (mm) & $0.13 \times 0.11 \times 0.09$ \\
\hline \multicolumn{2}{|l|}{ Data collection } \\
\hline Diffractometer & Bruker AXS D8 Quest CMOS diffractometer \\
\hline Absorption correction & $\begin{array}{l}\text { Multi-scan SADABS 2016/2: Krause, L., Herbst-Irmer, R., Sheldrick G.M. } \\
\text { \& Stalke D., J. Appl. Cryst. } 48 \text { (2015) 3-10 }\end{array}$ \\
\hline$T_{\min }, T_{\max }$ & $0.239,0.263$ \\
\hline $\begin{array}{l}\text { No. of measured, independent and } \\
\text { observed }[I>2 \mathrm{~s}(I)] \text { reflections }\end{array}$ & 64125, 16476, 9229 \\
\hline$R_{\text {int }}$ & 0.069 \\
\hline$(\sin \theta / \lambda)_{\max }\left(\AA^{-1}\right)$ & 0.668 \\
\hline \multicolumn{2}{|l|}{ Refinement } \\
\hline$R\left[F^{2}>2 \sigma\left(F^{2}\right)\right], w R\left(F^{2}\right), S$ & $0.073,0.190,1.02$ \\
\hline No. of reflections & 16476 \\
\hline No. of parameters & 910 \\
\hline No. of restraints & 720 \\
\hline $\mathrm{H}$-atom treatment & $\mathrm{H}$-atom parameters constrained \\
\hline$\Delta \rho_{\max }, \Delta \rho_{\min }\left(\mathrm{e} \AA^{-3}\right)$ & $1.10,-0.62$ \\
\hline
\end{tabular}

Computer programs: Apex3 v2016.9-0 (Bruker, 2016), SAINT V8.37A (Bruker, 2016), SHELXS97 (Sheldrick, 2008), SHELXL2016/6 (Sheldrick, 2015, 2016), SHELXLE Rev714 (Hübschle et al., 2011). 


\section{Refinement details:}

Both borolene units are disordered by rotation of the $\mathrm{O}-\mathrm{C}(\mathrm{Me} 2)-\mathrm{C}(\mathrm{Me} 2)-\mathrm{O}$ units around the $\mathrm{C}-\mathrm{B}$ bond axis. The disorder was tentatively refined as two-fold, but there are indications for additional minor disorder with additional borolene orientations. The geometries of all four moieties were restrained to be similar, and $U^{\mathrm{ij}}$ components of ADPs of disordered atoms were restrained to be similar for atoms closer to each other than $2.0 \AA$. Subject to these conditions the occupancy ratios refined to $0.602(5)$ to $0.398(5)$ for the unit involving B1, and 0.709(5) to 0.291(5) for that involving B2.

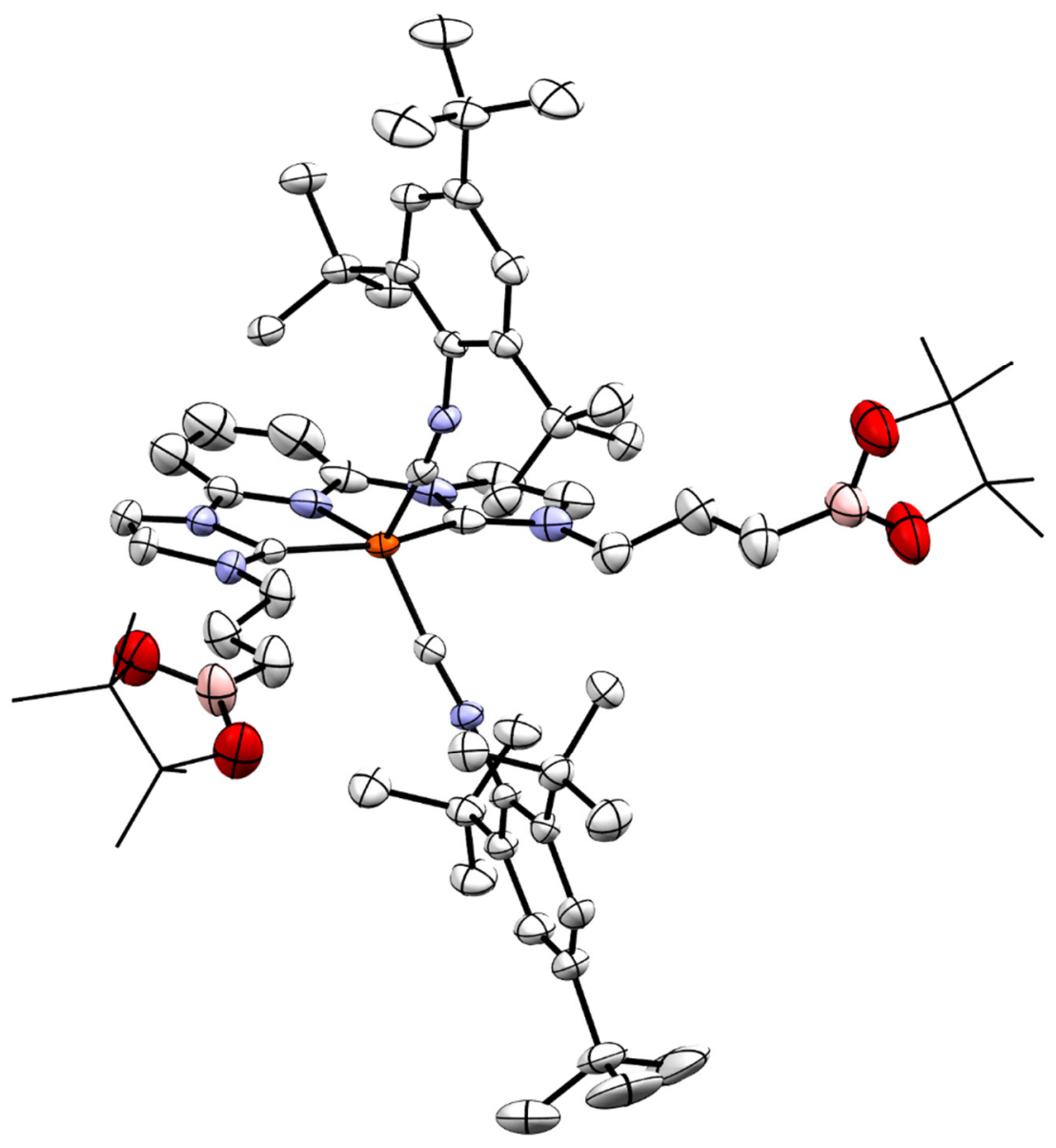

Figure $\mathbf{5 9 5}$ Molecular structure of $\left({ }^{\mathrm{BPin}} \mathrm{CNC}\right) \mathrm{Fe}\left(\mathrm{CN}\left(2,4,6-{ }^{t} \mathrm{Bu}_{3} \mathrm{C}_{6} \mathrm{H}_{2}\right)\right)_{2}\left(6-\mathrm{CN}^{t} \mathrm{Bu}_{3} \mathrm{C}_{6} \mathrm{H}_{2}\right)$ displayed with $50 \%$ probability ellipsoids. All $\mathrm{H}$-atoms are omitted and portions of the -BPin fragment are displayed in wireframe for improved clarity. Disordered fragments are omitted for clarity. 
Crystals of $\left({ }^{B P i n} \mathrm{CNC}\right) \mathrm{Fe}(\mathrm{CNPh})_{2}$ (6-CNPh) were obtained by layering a $\mathrm{C}_{6} \mathrm{H}_{6}$ solution of the molecule with hexamethyldisiloxane at room temperature. The crystals investigated were not of high enough quality to produce a publishable data set. Considerable streaking was observed between Bragg peaks and minimal high angle data was obtained. These resulted in large difference densities adjacent to the C-atoms attached to Fe ( $Q$ peak $>1.7 \mathrm{e}^{-}$). Because of this, the data was used solely to suggest connectivity. The modeled connectivity is consistent with the other spectroscopic data obtained ( $\left.{ }^{1} \mathrm{H} N M R,{ }^{13} \mathrm{C} \mathrm{NMR}, \mathrm{IR}\right)$.

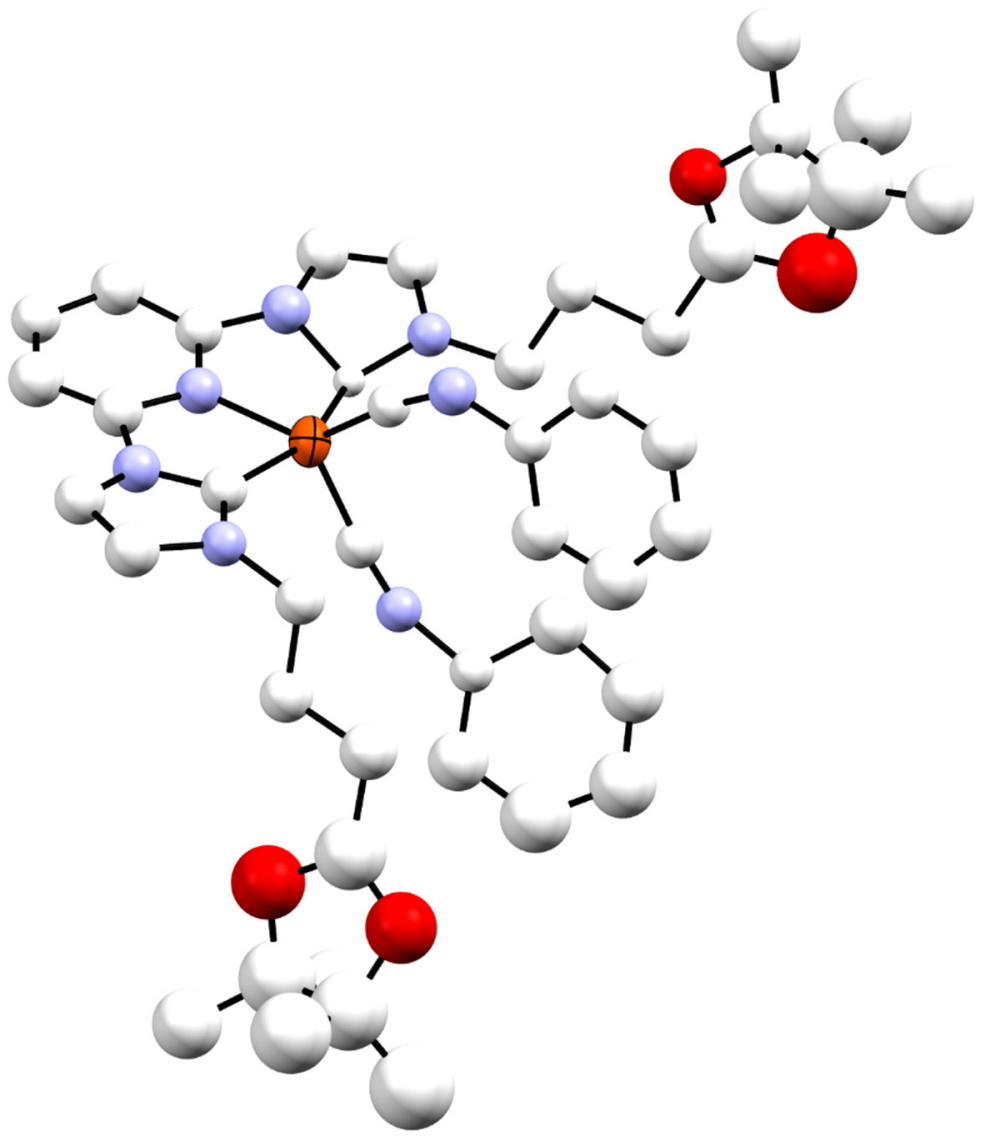

Figure S96 Low-quality molecular structure of $\left({ }^{\mathrm{BPin}} \mathrm{CNC}\right) \mathrm{Fe}(\mathrm{CNPh})_{2}(6-\mathrm{CNPh})$ used to establish probable connectivity. Due to poor data quality, further refinement was not pursued. Unit cell: $\mathrm{P} 2(1) / \mathrm{n} ; a, b, c=$ 22.95180, 7.60470, $25.75260 \AA \AA$; $\beta=110.7681^{\circ}$. 
Compound: $\left({ }^{\mathrm{BPin}} \mathrm{CNC}\right) \mathrm{Fe}\left(\mathrm{PMe}_{3}\right)_{2}\left(6-\mathrm{PMe}_{3}\right)$

Local Name: JK1174

CCDC Number: 2084281

Table S11. Crystallographic details for ( $\left.{ }^{\mathrm{BPin}} \mathrm{CNC}\right) \mathrm{Fe}\left(\mathrm{PMe}_{3}\right)_{2}\left(6-\mathrm{PMe}_{3}\right)$

\begin{tabular}{|c|c|}
\hline \multicolumn{2}{|l|}{ Crystal data } \\
\hline Chemical formula & $\mathrm{C}_{35} \mathrm{H}_{61} \mathrm{~B}_{2} \mathrm{FeN}_{5} \mathrm{O}_{4} \mathrm{P}_{2}$ \\
\hline$M_{r}$ & 755.29 \\
\hline Crystal system, space group & Orthorhombic, Pccn \\
\hline Temperature (K) & 85 \\
\hline$a, b, c(\AA)$ & 12.6605 (1), $14.2657(2), 22.0061$ (2) \\
\hline$V\left(\AA^{3}\right)$ & 3974.54 (7) \\
\hline$z$ & 4 \\
\hline Radiation type & $\mathrm{Cu} K \alpha$ \\
\hline$\mu\left(\mathrm{mm}^{-1}\right)$ & 4.14 \\
\hline Crystal size $(\mathrm{mm})$ & $0.13 \times 0.08 \times 0.06$ \\
\hline \multicolumn{2}{|l|}{ Data collection } \\
\hline Diffractometer & Abstract diffractometer \\
\hline Absorption correction & $\begin{array}{l}\text { Multi-scan CrysAlis PRO 1.171.38.41 (Rigaku Oxford Diffraction, 2015) } \\
\text { Empirical absorption correction using spherical harmonics, implemented } \\
\text { in SCALE3 ABSPACK scaling algorithm. }\end{array}$ \\
\hline$T_{\min }, T_{\max }$ & $0.829,1.000$ \\
\hline $\begin{array}{l}\text { No. of measured, independent and } \\
\text { observed }[I>2 s(I)] \text { reflections }\end{array}$ & $58330,3727,3637$ \\
\hline$R_{\text {int }}$ & 0.050 \\
\hline$(\sin \theta / \lambda)_{\max }\left(\AA^{-1}\right)$ & 0.607 \\
\hline \multicolumn{2}{|l|}{ Refinement } \\
\hline$R\left[F^{2}>2 \sigma\left(F^{2}\right)\right], w R\left(F^{2}\right), S$ & $0.049,0.138,1.10$ \\
\hline No. of reflections & 3727 \\
\hline No. of parameters & 231 \\
\hline $\mathrm{H}$-atom treatment & $\mathrm{H}$-atom parameters constrained \\
\hline$\Delta \rho_{\max }, \Delta \rho_{\min }\left(\mathrm{e} \AA^{-3}\right)$ & $0.84,-0.50$ \\
\hline
\end{tabular}

Computer programs: CrysAlis PRO 1.171.38.41 (Rigaku OD, 2015), SHELXS97 (Sheldrick, 2008),

SHELXL2014/7 (Sheldrick, 2014). 


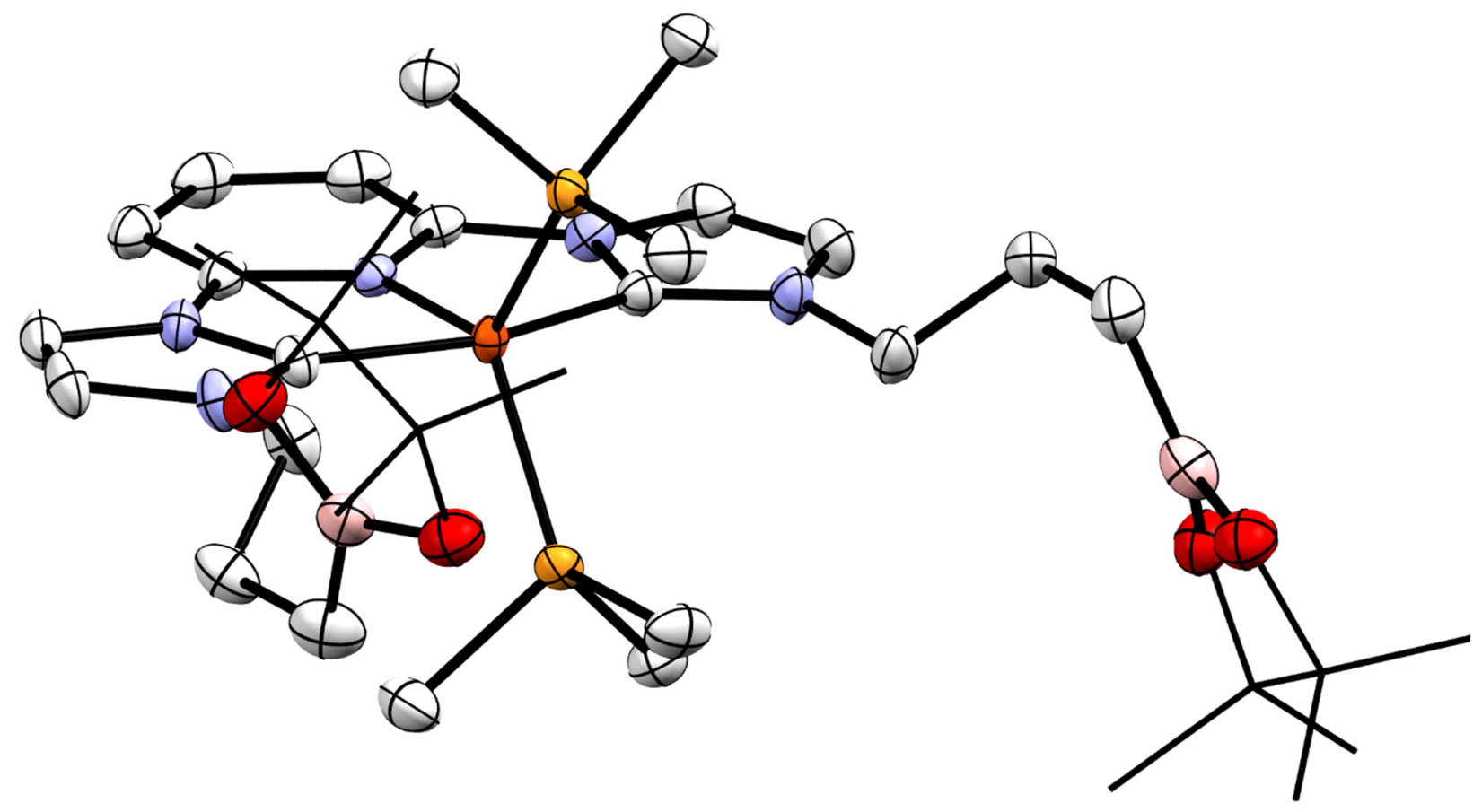

Figure $\mathbf{S 9 7}$ Molecular structure of $\left({ }^{\mathrm{BPin}} \mathrm{CNC}\right) \mathrm{Fe}\left(\mathrm{PMe}_{3}\right)_{2}$ (6-PMe $\left.\mathbf{P}_{3}\right)$ displayed with $50 \%$ probability ellipsoids. All $\mathrm{H}$-atoms are omitted for clarity. Portions of the -BPin fragments are displayed in wireframe for clarity. 
Compound: cis-( $\left.{ }^{\text {allyl }} \mathrm{CNC}\right) \mathrm{Fe}(\mathrm{CO})_{2}\left(\mathrm{C}_{6} \mathrm{~F}_{4}-\mathrm{BF}\left(\mathrm{C}_{6} \mathrm{~F}_{5}\right)_{2}\right)$

Local Name: JK2243

CCDC Number: 2084287

Table S12. Crystallographic details for cis-( $\left.{ }^{\text {allyl }} \mathrm{CNC}\right) \mathrm{Fe}(\mathrm{CO})_{2}\left(\mathrm{C}_{6} \mathrm{~F}_{4}-\mathrm{BF}\left(\mathrm{C}_{6} \mathrm{~F}_{5}\right)_{2}\right)$

\begin{tabular}{|c|c|}
\hline \multicolumn{2}{|l|}{ Crystal data } \\
\hline Chemical formula & $\mathrm{C}_{37} \mathrm{H}_{17} \mathrm{BF}_{15} \mathrm{FeN}_{5} \mathrm{O}_{2}$ \\
\hline$M_{\mathrm{r}}$ & 915.22 \\
\hline Crystal system, space group & Trigonal, $R \overline{3}: H$ \\
\hline Temperature (K) & 150 \\
\hline$a, c(\AA ̊)$ & $32.831(2), 18.5796(11)$ \\
\hline$V\left(\AA^{3}\right)$ & $17343(2)$ \\
\hline$z$ & 18 \\
\hline Radiation type & Mo $K \alpha$ \\
\hline$\mu\left(\mathrm{mm}^{-1}\right)$ & 0.51 \\
\hline Crystal size $(\mathrm{mm})$ & $0.09 \times 0.09 \times 0.08$ \\
\hline \multicolumn{2}{|l|}{ Data collection } \\
\hline Diffractometer & Bruker AXS D8 Quest CMOS diffractometer \\
\hline Absorption correction & $\begin{array}{l}\text { Multi-scan SADABS 2016/2: Krause, L., Herbst-Irmer, R., Sheldrick } \\
\text { G.M. \& Stalke D., J. Appl. Cryst. } 48 \text { (2015) 3-10 }\end{array}$ \\
\hline$T_{\min }, T_{\max }$ & $0.637,0.746$ \\
\hline $\begin{array}{l}\text { No. of measured, independent and } \\
\text { observed }[I>2 s(I)] \text { reflections }\end{array}$ & $68134,9530,6265$ \\
\hline$R_{\text {int }}$ & 0.091 \\
\hline$(\sin \theta / \lambda)_{\max }\left(\AA^{-1}\right)$ & 0.667 \\
\hline \multicolumn{2}{|l|}{ Refinement } \\
\hline$R\left[F^{2}>2 \sigma\left(F^{2}\right)\right], w R\left(F^{2}\right), S$ & $0.050,0.139,1.05$ \\
\hline No. of reflections & 9530 \\
\hline No. of parameters & 550 \\
\hline \multirow[t]{2}{*}{$\mathrm{H}$-atom treatment } & $\mathrm{H}$-atom parameters constrained \\
\hline & $\begin{array}{l}w=1 /\left[\mathrm{s}^{2}\left(F_{\mathrm{o}}^{2}\right)+(0.0611 P)^{2}+27.7893 P\right] \\
\text { where } P=\left(F_{\mathrm{o}}^{2}+2 F_{\mathrm{c}}^{2}\right) / 3\end{array}$ \\
\hline$\Delta \rho_{\max }, \Delta \rho_{\min }\left(\mathrm{e} \AA^{-3}\right)$ & $0.58,-0.43$ \\
\hline
\end{tabular}

Computer programs: Apex3 v2016.9-0 (Bruker, 2016), SAINT V8.37A (Bruker, 2016), SHELXS97

(Sheldrick, 2008), SHELXL2017/1 (Sheldrick, 2017), SHELXLE Rev859 (Hübschle et al., 2011). 


\section{Refinement details:}

The structure contains six independent solvent accessible voids of $2159 \AA^{3}$ combined, arranged around the three-fold axis. No substantial electron density peaks were found in the solvent accessible voids (less than $1.2 \mathrm{e}^{-} / \AA^{3}$ ) and the residual electron density peaks were not arranged in an interpretable pattern. The cif and fcf files were thus corrected using reverse Fourier transform methods using the SQUEEZE routine (P. van der Sluis \& A.L. Spek, Acta Cryst. 1990, A46, 194-201) as implemented in the program Platon. The resultant files were used in the further refinement. (The FAB file with details of the SQUEEZE results is appended to the cif file). The SQUEEZE procedure corrected for 329 electrons within the solvent accessible voids.

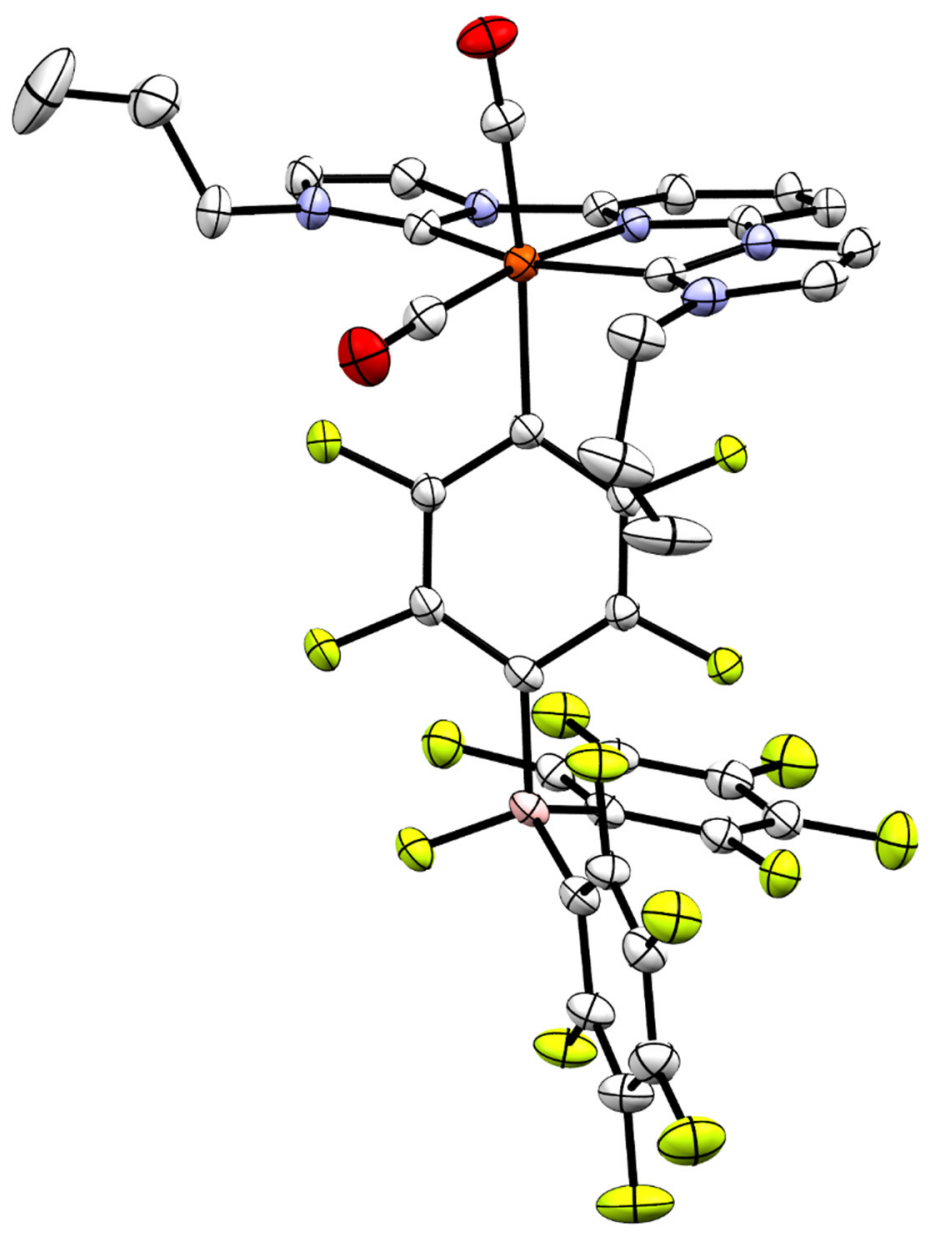

Figure S98 Molecular structure of cis-( $\left.{ }^{\text {allyl }} \mathrm{CNC}\right) \mathrm{Fe}(\mathrm{CO})_{2}\left(\mathrm{C}_{6} \mathrm{~F}_{4}-\mathrm{BF}\left(\mathrm{C}_{6} \mathrm{~F}_{5}\right)_{2}\right)$ displayed with $50 \%$ probability ellipsoids. All $\mathrm{H}$-atoms are omitted for clarity. Selected bond distances and angles: Fe- $\mathrm{C}_{\text {carbene }}=1.967(3)$, 1.969(2) $\AA$; Fe-CO (trans to pyridine) = 1.769(3) $\AA$; Fe-CO (cis to pyridine) $1.814(3) \AA ̊$ Fe- $\mathrm{N}_{\text {pyr }}=1.950(2) \AA$; $\mathrm{Fe}-\mathrm{C}_{6} \mathrm{~F}_{4} \mathrm{R}=2.071(3) \AA \AA \mathrm{B}-\mathrm{F}=1.444(3) ; \Sigma \mathrm{B} \alpha=323.5(2)^{\circ}$. 
Table S13. Experimentally determined bond distances $(\AA)$ and angles $\left(^{\circ}\right)$ of phosphine containing complexes.

\begin{tabular}{|c|c|c|c|c|}
\hline & $\begin{array}{l}\left({ }^{\text {allyl }} \mathrm{CNC}\right) \mathrm{Fe}\left(\mathrm{PMe}_{3}\right)_{2} \\
\left(3-\mathrm{PMe}_{3}\right)\end{array}$ & $\begin{array}{l}\text { (allyl } \mathrm{CNC}) \mathrm{Fe}\left(\mathrm{PEt}_{3}\right)_{2} \\
\left(3-\mathrm{PEt}_{3}\right)\end{array}$ & $\begin{array}{l}\text { ( }{ }^{\text {allyl } C N C) F e(d e p e) ~} \\
\text { (3-depe) }\end{array}$ & $\begin{array}{l}\left({ }^{\mathrm{BPin}} \mathrm{CNC}\right) \mathrm{Fe}\left(\mathrm{PMe}_{3}\right)_{2} \\
\left(6-\mathrm{PMe}_{3}\right)\end{array}$ \\
\hline Fe- $\mathrm{C}_{\text {carbene }}$ & $1.898(8)$ & $1.900(3)$ & $1.926(6)$ & $1.891(3)$ \\
\hline Fe- $\mathrm{C}_{\text {carbene }}$ & $1.895(8)$ & $1.903(3)$ & $1.923(6)$ & -- \\
\hline Fe- $\mathrm{N}_{\text {pyridine }}$ & $1.878(7)$ & $1.892(2)$ & $1.900(6)$ & $1.886(3)$ \\
\hline Fe-P & $2.169(2)$ & $2.1861(7)$ & $2.1640(15)$ & $2.1718(7)$ \\
\hline Fe-P & $2.174(2)$ & $2.2002(7)$ & $2.1524(14)$ & -- \\
\hline$\Sigma \mathrm{Ba}$ & -- & -- & -- & $359.9(3)$ \\
\hline$\tau_{5}$ & 0.545 & 0.593 & 0.328 & 0.574 \\
\hline P-Fe-P & 111.09(9) & $117.68(3)$ & $89.55(6)$ & $112.41(4)$ \\
\hline
\end{tabular}

Table S14. Experimentally determined bond distances $(\AA ̊)$ and angles $\left({ }^{\circ}\right)$ of isocyanide containing complexes.

\begin{tabular}{|c|c|c|c|c|c|}
\hline & $\begin{array}{l}\left({ }^{\text {allyl }} \mathrm{CNC}\right) \mathrm{Fe}(\mathrm{CNR})_{2} \\
\left(\mathrm{R}={ }^{t} \mathrm{Bu}\right) \\
\left(4-^{-} \mathrm{Bu}\right)\end{array}$ & $\begin{array}{l}\text { (allyl CNC)Fe(CNR) } 2 \\
(\mathrm{R}=\mathrm{Ph}) \\
\text { (4-Ph) }\end{array}$ & 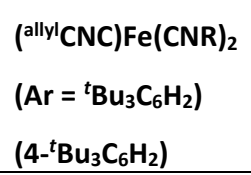 & $\begin{array}{l}\left({ }^{\mathrm{BPin}} \mathrm{CNC}\right) \mathrm{Fe}(\mathrm{CNR})_{2} \\
\left(\mathrm{Ar}={ }^{t} \mathrm{Bu}_{3} \mathrm{C}_{6} \mathrm{H}_{2}\right) \\
\left(6-{ }^{t} \mathrm{Bu}_{3} \mathrm{C}_{6} \mathrm{H}_{2}\right)\end{array}$ & $\begin{array}{l}\left({ }^{\mathrm{BBN}} \mathrm{CNC}\right) \mathrm{Fe}(\mathrm{CNR})_{2} \\
\left(\mathrm{Ar}={ }^{t} \mathrm{Bu}_{3} \mathrm{C}_{6} \mathrm{H}_{2}\right) \\
\left(5-{ }^{t} \mathrm{Bu}_{3} \mathrm{C}_{6} \mathrm{H}_{2}\right)\end{array}$ \\
\hline Fe- $\mathrm{C}_{\text {carbene }}$ & $1.9092(16)$ & $1.931(3)$ & $1.913(3)$ & $1.912(2)$ & $1.9113(9)$ \\
\hline Fe- $\mathrm{C}_{\text {carbene }}$ & -- & $1.904(3)$ & $1.926(4)$ & $1.918(3)$ & $1.9081(11)$ \\
\hline Fe- $N_{\text {pyridine }}$ & $1.914(2)$ & $1.907(4)$ & $1.890(4)$ & $1.907(3)$ & $1.9283(11)$ \\
\hline Fe-CNR & $1.8018(17)$ & $1.738(4)$ & $1.798(3)$ & $1.787(3)$ & $1.7966(11)$ \\
\hline Fe-CNR & -- & $1.796(3)$ & $1.793(2)$ & $1.801(3)$ & $1.8004(11)$ \\
\hline C- $\mathbf{N}_{\text {isocyanide }}$ & $1.198(2)$ & $1.236(5)$ & $1.196(3)$ & $1.196(3)$ & $1.1889(14)$ \\
\hline C-N $\mathrm{N}_{\text {isocyanide }}$ & -- & $1.173(5)$ & $1.188(3)$ & $1.192(3)$ & $1.1947(14)$ \\
\hline$\Sigma B \alpha$ & -- & -- & -- & $359.9(4)$ & $359.82(11)$ \\
\hline$\Sigma B \alpha$ & -- & -- & -- & $359.9(4)$ & $360.00(13)$ \\
\hline$\tau_{5}$ & 0.498 & 0.327 & 0.24 & 0.406 & 0.308 \\
\hline C-Fe-C isocyanide $_{\text {e }}$ & $105.93(11)$ & $102.91(15)$ & $106.68(11)$ & $107.78(12)$ & $108.67(5)$ \\
\hline Fe-C-Nisocyanide & $175.29(15)$ & $170.6(3)$ & $174.1(2)$ & $175.3(2)$ & $173.77(9)$ \\
\hline $\mathrm{Fe}-\mathrm{C}-\mathrm{N}_{\text {isocyanide }}$ & -- & $177.0(3)$ & $178.8(2)$ & $173.1(2)$ & $178.34(10)$ \\
\hline C-N-C isocyanide & $142.35(17)$ & 133.9(3) & $165.9(2)$ & 162.6(3) & $172.80(11)$ \\
\hline C-N-C isocyanide & -- & 175.9(4) & $167.4(2)$ & 172.1(3) & $166.05(11)$ \\
\hline
\end{tabular}




\title{
$\underline{\text { References }}$
}

\author{
${ }^{1}$ Kiernicki, J. J.; Zeller, M.; Szymczak, N.K., Requirements for Lewis Acid-Mediated Capture and N-N Bond Cleavage \\ of Hydrazine at Iron. Inorg. Chem. 2019, 58, 1147-1154. \\ ${ }^{2}$ Chakraborty, S.; Chattopadhyay, J.; Guo, W.; Billups, W. E., Functionalization of Potassium Graphite. Angew. \\ Chem. Int. Ed. 2007, 46, 4486-4488. \\ ${ }^{3}$ Zakrzewski, J.; Krawczyk, M., Synthesis and Pesticidal Properties of Thio and Seleno Analogs of Some Common Urea \\ Herbicides. Phosphorus Sulfur Silicon Relat. Elem. 2009, 184, 1880-1903. \\ ${ }^{4}$ Longobardi, L.E.; Johnstone, T.C.; Falconer, R.L.; Russell, C.A.; Stephan, D.W., Hydroboration of Phosphaalkynes by \\ $\mathrm{HB}\left(\mathrm{C}_{6} \mathrm{~F}_{5}\right)_{2}$. Chem. Eur. J. 2016, 22, 12665. \\ ${ }^{5}$ Brookhart, M.; Grant, B.; Volpe, A. F., [(3,5- $\left.\left.\left(\mathrm{CF}_{3}\right)_{2} \mathrm{C}_{6} \mathrm{H}_{3}\right)_{4} \mathrm{~B}\right]^{-}\left[\mathrm{H}\left(\mathrm{OEt}_{2}\right)_{2}\right]^{+}$: A Convenient Reagent for Generation and \\ Stabilization of Cationic, Highly Electrophilic Organometallic Complexes. Organometallics 1992, 11, $3920-3922$. \\ ${ }^{6}$ Ohmiya, H.; Yokobori, U.; Makida, Y.; Sawamura, M., Copper-Catalyzed Y-Selective Allyl-Alkyl Coupling between \\ Allylic Phosphates and Alkylboranes. J. Am. Chem. Soc. 2010, 132, 2895-2897. \\ ${ }^{7}$ Bruker Advanced X-ray Solution, Apex3, SAINT, SADABS, Bruker AXS Inc.: Madison (WI), USA, 2018. \\ ${ }^{8}$ SHELXTL suite of programs, Version 6.14, 2000-2003, Bruker Advanced X-ray Solutions, Bruker AXS Inc., Madison, \\ Wisconsin: USA \\ ${ }^{9}$ Sheldrick, G. M., A Short History of SHELX. Acta Crystallogr., Sect. A. 2008, 64, 112-122. \\ ${ }^{10}$ Sheldrick, G.M. Crystal structure refinement with SHELXL. Acta Crystallogr. Sec.t C. Struct. Chem. 2015, 71, 3-8. \\ ${ }^{11}$ Hübschle, C.B., Sheldrick, G.M., Dittrich, B. ShelXle: a Qt graphical user interface for SHELXL. J. Appl. Crystallogr.
} 2011, 44, 1281-1284.

12 Danopoulos, A. A.; Wright, J. A.; Motherwell, W. B., Molecular $\mathrm{N}_{2}$ complexes of iron stabilised by N-heterocyclic 'pincer' dicarbene ligands. Chem. Commun. 2005, 784-786.

13 Danopoulos, A. A.; Tsoureas, N.; Wright, J. A.; Light, M. E., N-Heterocyclic Pincer Dicarbene Complexes of Iron(II): C-2 and C-5 Metalated Carbenes on the Same Metal Center, Organometallics 2004, 23, 166-168.

${ }^{14}$ Similar complexes have been exploited for isotope exchange with arene solvents and likely proceed through hydride intermediates. See: (a) Rummelt, S. M.; Darmon, J. M.; Yu, R. P.; Viereck, P.; Pabst, T. P.; Turner, Z. R.; Margulieux, G. W.; Gu, S.; Chirik, P. J., Synthesis, Structure, and Hydrogenolysis of Pyridine Dicarbene Iron Dialkyl Complexes. Organometallics 2019, 38, 3159-3168; (b) Pony Yu, R.; Hesk, D.; Rivera, N.; Pelczer, I.; Chirik, P. J., Ironcatalysed tritiation of pharmaceuticals. Nature 2016, 529, 195-199; (c) Yu, R. P.; Darmon, J. M.; Semproni, S. P.; Turner, Z. R.; Chirik, P. J., Synthesis of Iron Hydride Complexes Relevant to Hydrogen Isotope Exchange in Pharmaceuticals. Organometallics 2017, 36, 4341-4343; (d) Corpas, J.; Viereck, P.; Chirik, P. J., C(sp $\left.{ }^{2}\right)-H$ Activation with Pyridine Dicarbene Iron Dialkyl Complexes: Hydrogen Isotope Exchange of Arenes Using Benzene- $d_{6}$ as a Deuterium Source. ACS Catal. 2020, 10, 8640-8647. A comparable reaction between $\left({ }^{\mathrm{DIPP}} \mathrm{CNC}\right) \mathrm{Fe}\left(\mathrm{N}_{2}\right)_{2}$ and $\mathrm{N}-$ benzylideneaniline afforded an Fe(II)-H species, see: Danopoulos, A. A.; Pugh, D.; Smith, H.; Saßmannshausen, J., Structural and Reactivity Studies of "Pincer" Pyridine Dicarbene Complexes of Fe0: Experimental and Computational Comparison of the Phosphine and NHC Donors. Chem. Eur. J. 2009, 15, 5491-5502.

${ }^{15}$ (a) Longobardi, L. E.; Johnstone, T. C.; Falconer, R. L.; Russell, C. A.; Stephan, D. W., Chem. Euro. J. 2016, 22, 1266512669; (b) Parks, D. J.; Piers, W. E.; Yap, G. P. A., Organometallics 1998, 17, 5492-5503.

${ }^{16}$ (a) Simonneau, A.; Turrel, R.; Vendier, L.; Etienne, M., Angew. Chem. Int. Ed. 2017, 56, 12268-12272; (b) Shanahan, J. P.; Szymczak, N. K., J. Am. Chem. Soc. 2019, 141, 8550-8556; (c) Buss, J. A.; VanderVelde, D. G.; Agapie, T., J. Am. Chem. Soc. 2018, 140, 10121-10125; (d) Fukuda, T.; Yoshimoto, T.; Hashimoto, H.; Tobita, H., Organometallics 2016, 35, 921-924.

${ }^{17}$ Miller, C. J.; Chadha, U.; Ulibarri-Sanchez, J. R.; Dickie, D. A.; Kemp, R. A., Polyhedron 2016, 114, $351-359$.

18 (a) Welch, G. C.; Prieto, R.; Dureen, M. A.; Lough, A. J.; Labeodan, O. A.; Höltrichter-Rössmann, T.; Stephan, D. W., Dalton Trans. 2009, 1559-1570; (b) Welch, G. C.; Cabrera, L.; Chase, P. A.; Hollink, E.; Masuda, J. D.; Wei, P.; Stephan, D. W., Dalton Trans. 2007, 3407-3414; (c) Theuergarten, E.; Bannenberg, T.; Walter, M. D.; Holschumacher, D.; Freytag, M.; Daniliuc, C. G.; Jones, P. G.; Tamm, M., Dalton Trans. 2014, 43, 1651-1662; (d) Döring, S.; Erker, G.; Fröhlich, R.; Meyer, O.; Bergander, K., Organometallics 1998, 17, 2183-2187; (e) Cui, P.; Comanescu, C. C.; Iluc, V. M., Chem. Commun. 2015, 51, 6206-6209. 
${ }^{19}$ Liu, Y.; Harlang, T.; Canton, S. E.; Chábera, P.; Suárez-Alcántara, K.; Fleckhaus, A.; Vithanage, D. A.; Göransson, E.; Corani, A.; Lomoth, R.; Sundström, V.; Wärnmark, K., Chem. Commun. 2013, 49, 6412-6414.

${ }^{20}$ (a) Darmon, J. M.; Pony Yu, R.; Semproni, S. P.; Turner, Z. R.; Stieber, S. C. E.; DeBeer, S.; Chirik, P. J., Electronic Structure Determination of Pyridine $\mathrm{N}$-Heterocyclic Carbene Iron Dinitrogen Complexes and Neutral Ligand Derivatives. Organometallics 2014, 33, 5423-5422. (b) Pony Yu, R.; Darmon, J. M.; Milsmann, C.; Margulieux, G. W.; Stieber, S. C. E.; DeBeer, S.; Chirik, P. J., Catalytic Hydrogenation Activity and Electronic Structure Determination of Bis(arylimidazol-2-ylidene)pyridine Cobalt Alkyl and Hydride Complexes. J. Am. Chem. Soc. 2013, 135, 13168-13184. 